\title{
A dual-trap optical tweezer approach to study emulsion droplet interactions
}

by

Marjorie Ronan Griffiths

A thesis

submitted to the Victoria University of Wellington in fulfilment of the requirements for the degree of

Doctor of Philosophy 



\begin{abstract}
Foods are a highly complex form of soft condensed matter. Their complexity arises from a number of interrelated factors including the natural heterogeneity of raw materials, intricate composition, and the subtle changes in molecular interactions and micro-structural arrangements dictated by food processing and storage. It is highly important to understand the forces dictating the food structure as the assembly and organisation of major structural entities (i.e biopolymers, droplets, bubbles, networks, and particles) are responsible for the foods stability, texture, flow properties and more inclusively their organoleptic properties.

The structural entities of foods exhibit numerous forms of self-organization and have significant structure complexity and dynamic behaviour on the mesoscopic length scales from 10 to 1000 nanometres. These dynamic weak interactions between the constituents define the organized state that ranges from simple spatial or temporal ordering to more intricate interactions making up the food microstructure. These interactions are often small in magnitude and are short ranged making them difficult to measure directly. Very few studies have been carried out on direct force measurements in foodstuffs.
\end{abstract}

The focus of this research was to develop a dual-trap optical tweezer method to directly measure interactions between micrometre colloidal particles and ultimately to design an apparatus where interactions between less homogeneous systems, such as emulsion droplets could directly measured as a function of separation. As the name suggests, optical tweezers provide the ability to control the position of particles 
using a focused laser beam. The general concept of this method is to immobilise two particles in two separate optical traps and step one particle closer to the other stationary particle in a controlled fashion. The droplet's movement is then recorded using a high-speed camera that provides near-to-real-time images of the particle's positions. The particle's positions are determined by a 3-D tracking algorithm developed in-house which determines the position of both particles to a precision of sub-pixel accuracy. The force exerted on each droplet (by the other one) can be extracted as it is proportional to the trap strength $(\mathrm{pN} / \mu \mathrm{m})$ and the displacement of the particle from the centre of the optical trap $(\mu \mathrm{m})$.

To demonstrate the optical tweezer method,the interactions between silica beads of a known size were measured as a function of bead separation. The measured force-distance curves agreed with the electrostatic component of the DLVO theory. Once the method was established it was applied at increasing salt concentrations (decreasing Debye lengths). Interestingly, a salt concentration was found beyond which the experimental data no longer agreed with the predictions of DVLO theory. Above $100 \mu \mathrm{M}$ sodium chloride the Debye length was reduced to less than the Brownian fluctuations of the particles in the traps, which then dominated the apparent repulsion by restricting their particle trajectories, masking the actual nature of the electrostatic interactions. This resulted in force curves which fitted the exponential function, however, the fitted decay constant bore no resemblance to the actual Debye length. A diffusion experiment was designed to demonstrate the ability to measure interactions in multiple environments using the same pair of beads (at low salt concentrations where Debye lengths are faithfully recovered). The evolution of force-displacement curves was measured as the local salt concentration changed owing to the diffusion of salt from the interface and the results obtained were shown to agree with predictions based on a standard diffusion formalism.

Applying the dual-trap optical tweezers method, successfully demonstrated with silica beads, to less homogeneous systems such as emulsion droplets presented challenges which showcased that emulsion design was critical as certain criteria had to be met in order to facilitate undertaking the tweezer experiments. These criteria include particle 
size $(1-3 \mu \mathrm{m})$, low polydispersity, and a reasonable refractive index mismatch between the droplet and continuous phase. In keeping with food systems a protein stabilised oil-in-water emulsion was chosen. Two popular emulsifiers, sodium caseinate and $\beta$-lactoglobulin, were investigated at different ionic strength, $\mathrm{pH}$ and homogenisation pressures and phase volumes. The emulsion chosen for direct force measurements was a sodium caseinate emulsion when prepared in a $100 \mathrm{mM}$ phosphate buffer at pH 7.0, 60 wt. \% soya bean oil and 0.04 wt.\% protein which provided an adequate droplet size with minimal polydispersity.

Interactions between pairs of sodium caseinate emulsion droplets were measured. Unlike for silica beads, the individual droplet size needed to be measured to determine the surface-to-surface separation of droplet pairs. The droplet's diameter was determined by measuring the restricted diffusion of the droplet in a weak optical trap and fitting the short time mean squared displacement behaviour to a Brownian motion simulation. It was found that the droplet size can be determined in this fashion to within $50 \mathrm{~nm}$.

Moving forward, the interactions between pairs of emulsion droplets were measured in water using the same method gleaned from the silica bead interaction study. The experimental data fitted well to the electrostatic force described by the DLVO theory with reasonable $\zeta$-potentials extracted. To further demonstrate this dual optical tweezer method, interactions between the same pair of droplets were measured at increasing $\mathrm{NaCl}$ concentrations by means of diffusion. The expected trend has found to agree from calculations of increased local salt concentration based on a diffusion equation. At salt concentrations above $100 \mu \mathrm{m}$ significant deviations in the force-curves were observed that may signal salt induced changes of the droplet's interface or be attributed to the small magnitude of the force being within the noise. This warrants further investigation.

In conclusion, the dual-trap optical tweezers have shown incredible potential to become a robust method to measure the interactions between droplets. This method has some clear advantages over current methods including that force, and spatial 
resolution is superior, sample preparation is straightforward, forces are measured in 3-dimensions, and the droplets are free in solution during measurement, not wetted on surfaces. Accordingly, dual-trap optical tweezer methodology has provided the ability to measure interactions to a precision that has not yet been achieved by any other method for the study of emulsion systems, which in itself is a major achievement. This method is another tool in the toolbox of a colloid chemist, food scientist and physicists to probe interactions in soft materials. 


\section{Acknowledgements}

This thesis would not have been possible without the guidance, friendship and support of a number of people.

First and foremost, I would like to thank my supervisors Kate McGrath and Bill Williams for their guidance, encouragement, patience, support, and believe in me. Kate, thank you for your unwavering support and allowing me to follow my own curiosities. Bill, I admire you for your curiosity and excitement, your dedication, perseverance and commitment to teaching.

Special thanks goes to Allan Raudsepp ("Allan the Machine"). Allan, you are one of the greatest scientist I have ever met, your attention to detail, thoroughness, and curiosity is something I admire. I believe I am a better scientist because of you; it has been a privilege to work beside you over the past four years. This thesis would not have the elegance it has if it was not for your creative coding and intellectual input.

A thank you to Brad and Steve for teaching me how to use the optical tweezers. Rob, for his insight on microfluidics, as well my labmates both in Palmerston North and in Wellington, Sandy, Pablo, Lisa, Natasha, Graham, Nurul, Mario, Mehrdad, Davoud, Saeideh and Christina, for good times in the lab. I would like to acknowledge the academic and supporting staff of Victoria University of Wellington, and at Massey University. I would like to specially thank the SCPS Workshop, Alan, Nick and Manu for your patients with building and repairing the homogeniser multiple times 
over the years. As well as the Pete the computer support at Massey University.

I would like to thank the Williams family, Mandy, Bill, Polly, Jim, and Dora, for your hospitality and sharing your home.

Special thanks to my friends and family, for being apart of this journey, for your company, laughter and support; Mom, Dad, Kirsten, Dan and Aidan. Also, I would like to thank Amy, Sonja, Britt, Mandy and Reya for making New Zealand feel not so far away.

Finally, I especially would like to thank my best friend and partner in crime, Stefan without you I could not have never finished. We will always fondly remember our times in New Zealand, as we shared so many adventures and this thesis was just one of them. Thank you for your encouragements and love. It has been fun, and now on to our next adventure. 


\section{Contents}

List of Figures $\quad$ xi

List of Tables $\quad$ xix

1. Direct Force Measurement Apparatuses $\quad 1$

1.1. A Historical Perspective . . . . . . . . . . . . . . . . . . . 3

1.2. Quantitative Advances ... . . . . . . . . . . . . . 4

1.2.1. Direct Methods . . . . . . . . . . . . . . . . . . 5

1.2.2. Indirect Methods . . . . . . . . . . . . . . . . . . . . 10

1.3. Motivation and Goals . . . . . . . . . . . . . . . . . . . . 12

1.4. Thesis Outline . . . . . . . . . . . . . . . . . . 13

2. Dual-Trap Optical Tweezers 14

2.1. Introduction and Key Literature . . . . . . . . . . . . . . . . . . . . 14

2.2. Optical Trapping Theory . . . . . . . . . . . . . . . . . . . . . . 19

2.2.1. Trapping Particulates . . . . . . . . . . . . . . . 20

2.3. The Apparatus . . . . . . . . . . . . . . . . . . . . 22

2.3.1. General Considerations . . . . . . . . . . . . . 22

2.3.2. Specific Set-up . . . . . . . . . . . . . . 27

2.4. Dual-Trap Methods . . . . . . . . . . . . . . . . . . 28

2.4.1. Sample Preparation . . . . . . . . . . . . . . 28

2.4.2. Microfluidics . . . . . . . . . . . . . . . . 29

2.4.3. Laser Configurations . . . . . . . . . . . . . . . . 39 
Contents

2.4.4. Fixed Trap Approach . . . . . . . . . . . . . . . . . 46

2.5. Direct Force Measurement Data Acquisition . . . . . . . . . . . . . . 47

2.5.1. Camera Settings . . . . . . . . . . . . . 47

2.5.2. Recording the Interaction Measurement . . . . . . . . . . . 48

2.6. Data Analysis for Direct Force Measurement . . . . . . . . . . . . . . 51

2.6.1. 3D Particle Tracking . . . . . . . . . . . . . . . . . 51

2.6.2. Empty Trap Measurement . . . . . . . . . . . . . . . . . 55

2.7. Summary . . . . . . . . . . . . . . . . . . . . . 58

3. Interactions between Silica Beads $\quad 59$

3.1. Introduction . . . . . . . . . . . . . . . . 6 60

3.1.1. Electrostatic Forces . . . . . . . . . . . . . . . . 60

3.1.2. $\zeta$-potential .................. 62

3.2. Experimental . . . . . . . . . . . . . . . . 62

3.3. Results . . . . . . . . . . . . . . . . . . . . . . . . . 64

3.3.1. Interactions Between Silica Beads in Water . . . . . . . . . . . 64

3.3.2. Interactions at Decreasing Debye-lengths . . . . . . . . . . 68

3.3.3. Interactions at $>100 \mu \mathrm{M} \mathrm{NaCl} \ldots \ldots . \ldots 70$

3.4. Discussion . . . . . . . . . . . . . . . . 74

3.5. Conclusion . . . . . . . . . . . . . . . . 76

4. Emulsion Introduction and Design $\quad 77$

4.1. Introduction . . . . . . . . . . . . . . . . 77

4.1.1. Emulsion Classification . . . . . . . . . . . . . . 78

4.2. Emulsion Formation . . . . . . . . . . . . . . . . . . 80

4.3. Interfacial Layer . . . . . . . . . . . . . . . . . . . . 82

4.3.1. $\boldsymbol{\beta}$-Lactoglobulin . . . . . . . . . . . . . 83

4.3.2. Caseins . . . . . . . . . . . . . . . 86

4.4. Mechanisms of Instability . . . . . . . . . . . . . . . . 88

4.4.1. Coalescence ... . . . . . . . . . . . . . 90

4.4.2. Creaming . . . . . . . . . . . . . . . . 91

4.4.3. Flocculation . . . . . . . . . . . . . . . . . . 92

viii 
4.4.4. Ostwald Ripening . . . . . . . . . . . . . . . . . . . . . . . . 92

4.5. Formulation \& Characterisation . . . . . . . . . . . . . . . . 95

4.5.1. Emulsion Criteria . . . . . . . . . . . . . . 95

4.6. Emulsion design . . . . . . . . . . . . . . . . . . 96

4.6.1. Materials . . . . . . . . . . . . . . 96

4.6.2. Emulsion Preparation . . . . . . . . . . . . . . . . . 97

4.7. Characterisation Techniques . . . . . . . . . . . . . . . . . 98

4.7.1. Droplet Size Distribution . . . . . . . . . . . . . . . . . 99

4.7.2. $\quad$-potential . . . . . . . . . . . . . . . 100

4.7.3. Microscopy . . . . . . . . . . . . . . . . 103

4.8. Results and discussion . . . . . . . . . . . . . . . . . . . 104

4.8.1. Effect of Homogenisation Pressure . . . . . . . . . . . . . . . . 104

4.8.2. Effect on Homogenisation Temperature . . . . . . . . . . . . . 108

4.8.3. Effect of Protein Concentration . . . . . . . . . . . . . . 109

4.8.4. Effect of Oil Concentration . . . . . . . . . . . . . . . . . 111

4.8.5. Effect of Ionic Strength . . . . . . . . . . . . . . . . . . . . 112

4.8.6. Droplet $\zeta$-potential . . . . . . . . . . . . 113

4.9. Conclusion . . . . . . . . . . . . . . . . . . . . . 114

5. Emulsion droplet interactions 116

5.1. Introduction . . . . . . . . . . . . . . . 116

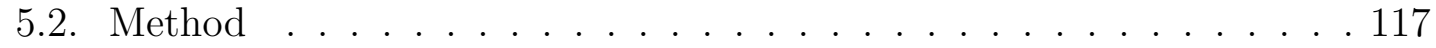

5.3. Results . . . . . . . . . . . . . . . . . . . . 118

5.3.1. Determining Droplet Size . . . . . . . . . . . . 118

5.3.2. Droplet Interactions in Water . . . . . . . . . . . . . . 121

5.3.3. Diffusion Experiment . . . . . . . . . . . . . . . 124

5.4. Discussion . . . . . . . . . . . . . . . . 127

5.5. Conclusion . . . . . . . . . . . . . . . . . . 131

6. Concluding Remarks and Future Work 132

$\begin{array}{ll}\text { A. Droplet Size Uncertainties } & 135\end{array}$ 
Contents

B. Interaction Measurement Definitions 138

$\begin{array}{ll}\text { C. Video Tracking Method } & 140\end{array}$ 


\section{List of Figures}

2.1. A description of the axial and lateral trapping forces by the Ray Optics model. The red arrows depict the direction of the laser where an increased light intensity is represented in a bold line. The blue arrows represents the resulting force where an increase in the magnitude of the force is represented as a bolded line. . . . . . . . . . . . . . 20

2.2. Example of the images of particles and droplets traped at $1 \mathrm{~W}$ by the spatial light modulator Spatial Light Modulator (SLM) laser. The images were taken at an exposure time of $0.002 \mathrm{~s}$ and frame rate for 250 fps. The region of interest (ROI) was 100 pixels x 100 pixels except for the $750 \mathrm{~nm}$ polystyrene bead that a black box was added to maintain the ROI. . . . . . . . . . . . . . . . . . . . . . 23

2.3. Diagram of the HOT apparatus used in this research. . . . . . . . 26

2.4. A diagram depicting how the trap strength is related to the force. Like a spring, an optical trap has a stiffness. The force acting on a colloidal particle is proportional to the trap strength. . . . . . . . . 27

2.5. Microfluidic set-up using hydrostatics to exchange fluid while beads were optically trapped. . . . . . . . . . . . . . . . 31 


\section{LIST OF FIGURES}

2.6. Schematic of the optical tweezer chip designs discussed for changing the aqueous phase surrounding the beads/droplets. . . . . . . . . . . 33

2.7. Schematic of the flow cell used in these experiments, an actual image of the chip with the port-bond connectors attached, in a customized chip holder. A side view of the attachment of the connectors onto the chip. . . . . . . . . . . . . . . . . . . 35

2.8. Schematic of the injection method to create a salt gradient within a channel. (1) The chip is filled with a water and bead mixture, and the syringe is detached. (2) The food dye is added to a desired distance, the amount added is noted. (3) The open channel is sealed (4) The food dye syringe is removed than the channel sealed. The chip is labelled where the water section begins. . . . . . . . . . . . . . 38

2.9. Shows the progression of trap sequences for the dual-SLM approach. The holding traps are shown in dashed lines. The bold traps are strong traps. The region of interest for the interaction sequence is given in green, reference sequence in blue and the mean-squared displacement (msd) in red. The bead's translation to and from the hold traps is represented by arrows. . . . . . . . . . . . . . . . . . . 44

2.10. Shows the progression of step sequences in the $y$-direction. Large evenly spaced traps were the first third of the sequence collecting 10k-20k frames per step, to investigative the correlations between the particle's movement. The last two thirds for the step sequence involved stepping the particle at more, smaller steps to resolve the force-displacement curve. . . . . . . . . . . . . . . . . . . . . . . . . . 44 
2.11. Plot demonstrating the chaotic motion of the traps when the traps are at close separations. The different data points represent repetitions of the same set of emulsion droplets. It is shown that regardless of the pair of emulsion droplets used the same motion of the optical trap is observed. . . . . . . . . . . . . . . . . . . . 45

2.12. Plot showing the trap sequence of the moving bead for an interaction measurement. The bead was stepped in the $y$-direction, where the bead was stepped 240 steps at step sizes of 0.05 SLM units. . . . . . . 49

2.13. Plot showing the reference sequence where the beads were held in the $x$ and $y$-directions and stepped in the $z$-direction in a saw-tooth manner. The bead was stepped in increments of 1 SLM unit from -26 to +8 SLM units, if the interaction measurement was recorded at $z=-9$ SLM units. . . . . . . . . . . . . . . . . . 50

2.14. Row 1: The particle positions of the moving bead (blue) and the stationary bead (red) are smoothed, and de-drifted. Row 2: Moving standard deviation Row 3: Bead displacement. Row 4: Force in the $x-, y$-and $z$-directions. . . . . . . . . . . . . 53

2.15. Bead positions in the $x-, y-$ and $z$-directions of an interaction measurement. The moving bead (top row) and the stationary trap (bottom row), note the y-axis scales. . . . . . . . . . . 54

2.16. Plot showing an example of an empty trap measurement. Row 1: tracked data of the stationary bead. Row 2: The de-drifted and smoothed positions. Row 3: The cubic spline fit of the smoothed and de-drifted data. . . . . . . . . . . . . . . . . . . . 5 56

2.17. Illustration of the bead separation. . . . . . . . . . . . . . . 57 


\section{LIST OF FIGURES}

2.18. Images of silica beads when well separated (left image). Middle image shows the result of removing the background, while maintaining the integrity of the beads image. Right images shows the calculated centre of mass. . . . . . . . . . . . . . . . . . . . . . . . . . 58

3.1. Visualisation of the interaction between two silica beads in water in a well slide. Bead $B$ is stepped toward bead $A$ between $T_{1}$ and $T_{3}$, then stepped away $T_{3}$ and $T_{5}$. Upon bead $B$ approaching bead $A$ the beads did show some z-movement. . . . . . . . . . . . . . . . . . 64

3.2. Tracked positions of the moving bead (bottom row) and the stationary bead (top row) in the $x-, y-$ and $z$-positions. The tracked data have been corrected for drift and smoothed over the frame rate $\left(250^{-1} \mathrm{~s}\right)$. (Note the different y-scales) . . . . . . . . . . . . . 66

3.3. Measured force-displacement curve of silica beads $(2 r=1.85 \mu \mathrm{m})$ in a well slide in water. The data were fitted to Equation 3.5, resulting in a Debye length of $109 \mathrm{~nm}$, charge on the droplet $(Z)$ of $3.39 \times 10^{9}$ and an ionic strength of $7.82 \times 10^{-6} \mathrm{M} \ldots \ldots 68$

3.4. Force-displacement curves from 100-5000 $\mu \mathrm{M}$. . . . . . . . . . . . . . 69

3.5. The decay constants extracted from the force-displacement curves at its corresponding prepared salt concentration. It can be seen that the fitted Debye length (०), aligns with the nominal Debye length $(\bullet)$ at prepared $\mathrm{NaCl}$ concentrations of $0.01 \mathrm{mM}$ and $0.1 \mathrm{mM}$. At prepared concentrations above $0.1 \mathrm{mM}$, the decay constant follows suit with the RMSD $(\square) . \ldots \ldots \ldots$. . . . . . . . . . . . . . . 70 
3.6. Image of the microfludic chip used for this diffusion experiment. Water and beads were added to the right side of the chip and a $5 \mathrm{mM} \mathrm{NaCl}$ solution was added to the left side of the chip creating an interface at $1.29 \mathrm{~cm}$. (The beads were trapped where the objective is positioned in the photograph.) The chip was sealed to ensure a static environment. 71

3.7. Force displacement curves of silica beads as a function of time as the local salt concentration was increasing away from the salt-water interface. 13 measurements were taken over 2.8 hours. Left plot shows the interaction data in colour and their corresponding fits in solid black lines. For simplicity, the right plot shows only the fit data. . . . 72

3.8. The salt concentration extracted from the time-resolved force curves as a function of time. The diffusion formalism shows excellent agreement with the measured data. . . . . . . . . . . . . . . . . 74

4.1. Schematic of a oil-in-water emulsion. . . . . . . . . . . . . . . . 78

4.2. Schematic of the effect of concentration of internal phase on the viscoelastic properties from dilute to concentrated emulsions. $\phi_{g}$ is the oil fraction where there is a glass transition state where the droplets become caged by their neighbours and $\phi_{R C P}$ is the oil fraction where droplets become randomly closed packed where the interfaces become

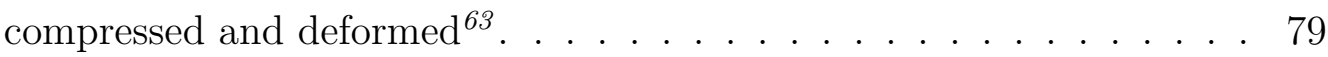

4.3. The influence of protein concentration during homogenisation on the unfolding of absorbed proteins. At low protein concentration, the absorbed $\beta$-lactoglobulin $(\beta$-lg) molecules have more time to unfold before the additional proteins absorb which leads to greater conformational changes. Figure reproduced with permission from Kim et al. $^{77} \ldots \ldots \ldots \ldots \ldots \ldots$ 


\section{LIST OF FIGURES}

4.4. Depiction of the 'train-loop-tail' model of $\beta$-casein (A), $\alpha_{s 1, s 2 \text {-casein }}$

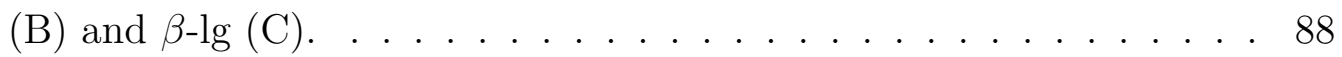

4.5. Illustration of the coalescence process in oil-in-water emulsions ${ }^{61} \ldots 89$

4.6. Illustration of the coalescence process in oil-in-water emulsions ${ }^{61}$. . 90

4.7. Creaming of an oil-in-water emulsion. . . . . . . . . . . . . . . . . 91

4.8. Illustration of bridging and depletion flocculation. . . . . . . . . . . 92

4.9. Illustration of the Ostwald ripening process. The large oil droplets grow at the expense of the smaller droplets ${ }^{87} \ldots \ldots$. . . . . . . 93

4.10. Avestin EmulsiFlex-C5 high-pressure homogenizer. . . . . . . . . . . . 98

4.11. Schematic of the MALVERN Mastersizer 2000, static light scattering instrument. . . . . . . . . . . . . . . . . . . . . . 99

4.12. Diagram of the electrical double layer of an emulsion droplet. . . . . . 102

4.13. Particle size distribution of Sodium Caseinate (sodium caseinate) (grey) and $\beta$-lg stabilised emulsion (black) homogenised at different conditions. An increase in homogenisation pressure from $34 \mathrm{MPa}, 103$ $\mathrm{MPa}$ and $206 \mathrm{MPa}$ is represented in rows 1-3. Samples were collected at 1, 3 and 5 passes, and this is represented by columns 1-3, respectively. 105

4.14. Particle size distribution of sodium caseinate (top row) and $\beta$-lg stabilised emulsion (bottom row) homogenised at $34 \mathrm{MPa}$ (left column) and $103 \mathrm{MPa}$ (right column) for 3 passes. . . . . . . . . . . . . . . 110 
4.15. Comparison between sodium caseinate and $\beta-\lg$ stabilised emulsions at protein concentrations in the aqueous phase of 1, 1.5 and 2 wt.\% protein, 60 wt.\% oil, $100 \mathrm{mM}$ phosphate buffer at $\mathrm{pH}$ 7.0. The emulsion was prepared at $103 \mathrm{MPa}$ for 3 passes through the homogeniser. The $d_{3,2}$ values decreased with an increase in protein concentration. . . . . 111

4.16. Comparison between sodium caseinate (left plot) and $\beta$-lg (right plot) stabilised emulsions at protein concentrations of $10 \mathrm{wt} . \%, 30 \mathrm{wt} . \%$ and 60 wt.\% oil in a phosphate buffer of $100 \mathrm{mM}, \mathrm{pH} 7.0$. . . . . . 112

4.17. Comparison between sodium caseinate (left plot) and $\beta$-lg (right plot) stabilised emulsions at ionic strengths of $1 \mathrm{mM}, 10 \mathrm{mM} 50 \mathrm{mM}$ and $100 \mathrm{mM}$ at $\mathrm{pH} 7.0 \ldots \ldots 113$

4.18. The $\zeta$-potential of sodium caseinate emulsions at increasing ionic strength at neutral $\mathrm{pH}$. The right plot shows the corresponding conductivity of the continuous phase. . . . . . . . . . . . . 114

5.1. Mean squared displacement of a $1.85 \mu \mathrm{m}$ silica bead. Plot (a) shows the uncalibrated MSD. Plot (b) shows the calibrated MSD (pixel to metre). Plot (c) shows the MSD model calculation (in black) overlaid onto the calibrated MSD. The $x$-data is in red, $y$-data in blue and $z$-data in green. . . . . . . . . . . . . . . . . . . . . 12

5.2. Mean squared displacement of two different emulsion droplets (left and right columns). The first row shows the uncalibrated MSD (subplots a \& d), middle row shows the calibrated MSD (subplots b \& e) and the third row shows the fits (in black) of the calibrated data (subplots c \& f). The $x$-data are in red, $y$ - data in blue and $z$-data in green. . . 121

5.3. Images of the emulsion droplets and silica beads. . . . . . . . . . . . . 122 


\section{LIST OF FIGURES}

5.4. Force displacement curves of three pairs of sodium caseinate stabilised oil droplets in water. Corresponding images of the interactions when the droplets are well-separated are shown beside their respective force curve. . . . . . . . . . . . . . . . . . . . 123

5.5. Force displacement curves of emulsion droplets as a function of time as the local salt concentration was increasing owing to the diffusion from a $5 \mathrm{mM}$ salt solution-water interface. Fifteen measurements were taken over 200 min. Left plot shows the interaction data in colour and their corresponding fits in solid black lines. For simplicity, the right plot shows only the fit data. . . . . . . . . . . . . 125

5.6. The salt concentration extracted from the time-resolved force curves as a function of time. The diffusion formalism is shown in red. Region I, is the region where excellent exponential fits and realistic $\zeta$-potentials are found and Region II shows anomalous behaviour, where the extracted salt concentration no longer trends with the diffusion formalism. Here the root mean squared thermal fluctuations as determined by trap strength interfere - but it is noteworthy that the curves are no longer exponential signally perhaps another behaviour as the average Debye length in fact is not representative of the expected trend. . . . . . . 126

A.1. (a) Mean squared displacement of an emulsion droplet in a weak optical trap at an exposure time of $1 \mathrm{~ms}$ fitted to a Brownian motion simulation with decreasing diffusion constants where the black line shows the experimental data. (b) The initial part of the data magnified.135

A.2. (a) MSD measurement of an emulsion droplet fitted to Brownian simulation. The fit calculations associated with decreasing trap strength are shown. Though, difficult to determine the trap strength to great accuracy, $0.7 \mathrm{pN} / \mu \mathrm{m}$ shows to be a reasonable fit to the experimental data. (b) The latter part of the data magnified. . . . . . . . . . . . 137 


\section{List of Tables}

2.1. A comparison of the Refractive Index of commonly studied colloidal particles in an aqueous environment used in optical tweezers (OT)

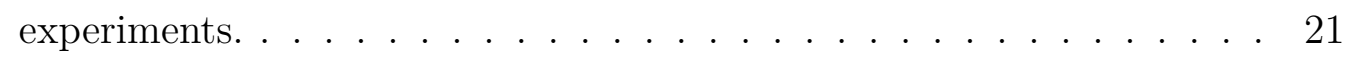

2.2. Dimensions of channel $I, I I$ and $I I I$ specified by the manufacturer . . 36

2.3. Example of the coordinates output for hologram generation. $\mathrm{X}$ and $\mathrm{Y}$, specify the position of the trap in the focal plan. Z, specifies the height of the trap, relative to the focal plane. Intensity, specifies the relative laser power intensities of the individual traps. Finally, charge is used to create optical vortices. Optical vortices are rings of light that can impart angular momentum to trapped objects. . . . . . . . 40

2.4. Camera settings and laser intensities for a single interaction measurement using the fixed trap approach. Background images were collected at the same settings for the Interaction, Reference and MSD measurements. Explanations of the measurement terms are found in Appendix B. . . . . . . . . . . . . . . . . . 49

3.1. Trap strengths for silica beads in water when the laser power is set to

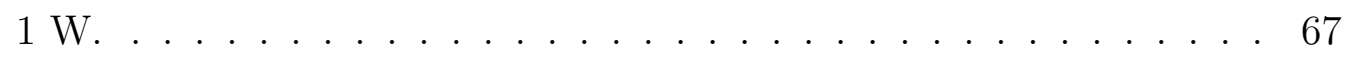




\section{LIST OF TABLES}

3.2. The results from fitting the force curves to Equation 3.5. The Debye length, corresponding salt concentration and charge on the bead is presented. The $R^{2}$ values show that the force curves had excellent fits. 73

4.1. A comparison of Macro and Microemulsions ${ }^{64} \ldots . . . . . . . .80$

4.2. Comparison of common homogenisers in the food industry . . . . . . 81

4.3. Summary of the amino acid variations of the three genetic variations of the $\beta$-lg. . . . . . . . . . . . . . . . . . . . . . 85

4.4. Average droplet size distributions of sodium caseinate and $\beta$-lg emulsion stabilised oil-in-water emulsions. Standard deviations of 6 measurements are represented in parentheses. Presented are the average $d_{3,2}$ diameter of droplets along with the span. An increase in the polydispersity of the emulsion increases the span number. A monodispersed emulsion will have a span value close to 1.0 . . . . . . . . 107

5.1. Diffusion constants $(D)$ and corresponding droplet radii $(a)$ from the fitted short time MSD behaviour for the three pairs of droplets presented in Figure 5.4. The Debye length $\left(\kappa^{-1}\right)$, salt concentration $(c)$ and charge on the droplet's surface $(Z)$ determined from the fitted force-separation curves. The $\zeta$-potential was calculated from Equation 3.6. The $R^{2}$ values show an excellent fit of the force-separation curves. Uncertainties were calculated from the $95 \%$ confidence bounds except for the droplet radii uncertainties where an explanation is presented in Appendix A. . . . . . . . . . . . . . . . . . . . . . . . . . . . . . . 124 
5.2. The results from fitting the force curves given in Figure 5.5 using Equation 3.5. The Debye length, corresponding salt concentration and charge on the bead are presented. Measurement numbers 1-5 result in true-force curves and measurements 6-15 is where the Debye length no longer results in strictly electrostatic behaviour. . . . . . . 127

A.1. Corresponding droplet sizes for the following diffusion constants . . . 136 



\section{List of Abbreviations}

$\begin{array}{ll}\text { AFM } & \text { Atomic force microscope } \\ \beta \text {-lg } & \text { Beta-lactoglobulin (milk protein) } \\ \lambda_{b} & \text { Bjerrum length } \\ \text { BSA } & \text { Bovine Serum Albumin } \\ \text { CCFA } & \text { Cantilevered-capillary force apparatus } \\ \text { COM } & \text { Centre of mass } \\ c_{b u l k} & \text { Concentration of ions } \\ \kappa^{-1} & \text { Debye length } \\ D & \text { Diffusion constant } \\ \text { DLS } & \text { Dynamic light scattering } \\ e & \text { Elementary charge } \\ \text { ET } & \text { Exposure time } \\ \text { FR } & \text { Frame rate } \\ \text { HOT } & \text { Holographic optical tweezer } \\ C_{i 0} & \text { Initial salt concentration } \\ \text { LSFA } & \text { Liquid surface force apparatus } \\ \text { MCT } & \text { Magnetic chaining technique } \\ m s d & \text { Mean squared displacement } \\ \text { MFX } & \text { Microfluidics } \\ \text { O/W } & \end{array}$




\section{LIST OF TABLES}

$\begin{array}{ll}\text { OT } & \text { Optical Tweezers } \\ e_{0} & \text { Permittivity of free space } \\ \text { QPD } & \text { Quadrant photo diode } \\ r \text { or } R & \text { Radius } \\ \text { ROI } & \text { Region of interest } \\ e_{r} & \text { Relative permittivity } \\ \text { STM } & \text { Scanning tunnelling microscope } \\ \text { SLM } & \text { Spatial light modulator } \\ \text { SLS } & \text { Static light scattering } \\ \text { SFA } & \text { Surface force apparatus } \\ k_{B} T & \text { Thermal energy } \\ Z & \text { Total charge } \\ k & \text { Trap strength } \\ \text { w/o } & \text { Water-in-oil } \\ w / o / w & \text { Water-in-oil-in-water } \\ \zeta & \zeta \text {-potential }\end{array}$

xxiv 



\section{Direct Force Measurement Apparatuses}

The interactions within and between molecules and materials dictate the physical properties of matter. Interactions between rigid, hard body materials are well understood at the atomic and molecular level and, therefore, their physiochemical behaviours can be predicted with reasonable certainty. Their properties are dependent on the basics of atomic theory, they are homogeneous throughout and are easily manipulated for experimental measurement allowing direct access to their fundamental response. Similarly, simple liquids are also well understood and characterized as their behaviour does not change under shear or stress providing relatively straightforward characterization. However, most materials exhibit properties somewhere between hard body, rigid materials and simple liquids; these are known as soft matter or complex fluids.

Soft matter cannot be simply defined at the atomic level. The constituents of these materials include polymers, surfactants, soft colloidal particles (bubbles, droplets) and biological materials (polysaccharides, DNA, protein). Such materials exhibit numerous forms of self-organization and have significant structure and dynamic behaviour on the mesoscopic length scales from 10 to $1000 \mathrm{~nm}$. Dynamic weak interactions between the constituents in soft matter materials define the organized state that ranges from simple spatial or temporal ordering to a more intricate interaction between order and function in biological systems. Examples of these 


\section{Direct Force Measurement Apparatuses}

systems include colloidal suspensions, ferrofluids, emulsions, surfactant assemblies, liquid crystals, bio-membranes, polymer solutions, gels and foams. The extent of these materials in industrial use cannot be underestimated. They are found in virtually every aspect of everyday life including medicines, foods, roads, tyres, plastics, and cosmetics.

There is considerable interest across a range of industries from food to oil recovery and biotechnology in understanding these systems at the supramolecular length scale. In particular there is a strong push towards gaining insight into how each individual constituent (oil droplet, bubble, polymer, cell, etc.) of the soft matter defines the physiochemical properties of the material, thus allowing control over the material properties and ultimately ensuring that predictability is achieved. For this to be possible the interactions between the constituents must be quantitatively determined, via, for example, direct measurement of the forces between them.

Direct force measurements have been achieved for a wide array of chemically and physically distinct rigid particles in an assortment of environments. However, working with the responsive, deformable constituents of soft materials is not straightforward. Challenges arise which include immobilizing the constituents, for example, how to manipulate fluid droplets in the case of emulsions, to control and measure the interactions between them as a function of distance. Furthermore, such droplets may deform, wet on surfaces, coalesce and/or aggregate. Beyond these challenges, droplets may also be less dense than the solvent and therefore buoyancy must also be considered. Additionally, Brownian motion must be compensated for since small droplets exhibit greater Brownian motion than larger droplets making them inherently more difficult to work with. Nonetheless, they are the major contributors in real systems. Finally, the droplets must be stable over the lifetime of the experiment. Over the past 20 years, these challenges have begun to be addressed, and apparatuses traditionally used to investigate rigid particles have been modified to allow measurement of the interactions between soft matter constituents.

In this chapter, we focus on reviewing the scientific advances that have been made in 


\subsection{A Historical Perspective}

the methods of measuring and visualizing the interactions between the constituents in soft condensed matter such as droplets and bubbles. In particular we will consider methods that allow quantitative force measurements such as force vs. distance and/or time between two individual potentially deformable particles to be obtained. However, methods that provide detailed qualitative observations are also included. Not to underestimate the importance of theoretical and computational work, which has been developed alongside the experimental efforts, but for brevity this chapter will not dwell on these advances.

\subsection{A Historical Perspective}

The intention of this chapter is not to be a historical review, but instead to focus on the scientific advances. However, a brief explanation of the history of direct force measurements will help put the current advances into context. There have been several excellent reviews on this topic with a review by Craig in 1997 on the historical timeline of the development of surface force techniques ${ }^{1}$, particularly noteworthy. A more recent review by Liang et al. who explores interactions between colloidal particles dispersed in liquid focusing on two techniques the Surface Force Apparatus (SFA) and Atomic Force Microscopy (AFM), provides a good background in that area $^{2}$.

Scientific curiosity has driven the exploration and understanding of surface forces. Until the 1950s, the idea of surface forces was only theoretically explained and indirectly measured. Published in 1952, Overbeek, and Sparnaay attempted to directly measure the interactions between two surfaces - highly polished glass and quartz plates. The careful construction of an apparatus provided control of the two plates at a separation of approximately $20 \mathrm{~nm}$. The separation between the two plates was monitored by the Newton interference colours and the forces were measured by the bending of a stiff spring where one plate was mounted. Although, Overbeek, and Sparnaay may not have been able to achieve high precision and accuracy in 


\section{Direct Force Measurement Apparatuses}

these experiments the challenges they faced are still very much relevant in today's experiments that include dust removal and experimental cleanliness. Derjaguin et al. followed the careful construction of Derjaguin and co-workers in 1954 was used to measure attractive van der Waals forces between a convex lens and a flat glass surface within a vacuum ${ }^{4,5}$. Using an electro-torsion balance they were able to measure the force while an optical technique was designed to measure the distance between the two glass surfaces. This novel method provided the infrastructure for the slow and steady progress toward sophisticated techniques available today. Twenty years on the SFA was developed by Tabor and Winterton ${ }^{6}$ and later developed further by Tabor and Israelachvili and Israelachvili and Adams ${ }^{7}$. Studies utilizing SFA have enabled significant advances in the field of colloid science; they have been utilized successfully to measure the interactions between two planar surfaces, as well as interactions between droplets and planar surfaces for the first time. During the 1980s, several other useful techniques were being developed including Atomic Force Microscopy, Total Internal Reflection Microscopy and Optical trapping. Over time, these instruments have become more versatile, pushing the boundaries to achieve direct measurement of interactions between any two particles, surfaces or droplets.

\subsection{Quantitative Advances}

Quantitatively measuring the surface forces between deformable droplets is experimentally very challenging. These surface forces are short ranged (nm) and are small in magnitude $(\mathrm{pN})$ which provides considerable opportunity for contamination. The direct force apparatuses have been initially designed for solid particle measurements, however, through scientific advances techniques have been adapted to measure the interactions between deformable droplets. These advances include immobilization techniques (through careful consideration of surface chemistry) for the attachment of droplets to surfaces, loading droplets with ferrofluids or utilization of focused laser beams. Scientists and engineers have also been pushing the boundaries to achieve greater resolution of distance and time. The quantitative techniques that 
are described in this chapter include surface force apparatus (SFA), atomic force microscopy (AFM), the magnetic chaining technique (MCT) and finally optical tweezers.

\subsubsection{Direct Methods}

\subsubsection{Surface Force Apparatus}

The SFA is unable to detect interactions between two droplets. However, it can be used to measure interactions between a droplet and either a liquid film or a solid surface. The SFA has brought new knowledge to the field of colloid and surface chemistry as electrostatic forces, van der Waals attraction, hydration and structural forces have been investigated with high precision ${ }^{2}$. The first SFA was introduced in $1969^{6}$. There have been many variations of the SFA with modifications via the addition of a capillary allowing the formation of a mercury droplet or a bubble and the interactions between a mica surface and droplets have been measured. These were some of the first soft matter interactions measured. Using the capillary method the interactions between a droplet of mercury and a mica surface as well as a bubble and a mica surface were measured. This modified apparatus is now referred to as the liquid surface force apparatus (LSFA).

The original apparatus developed by Tabor and Winterton in 1968 was designed to measure the interactions between two smooth mica surfaces which were adhered to two cylindrical rods fixed at a 90 degree cross, this geometry was chosen as it modelled a sphere-flat arrangement ${ }^{6}$. The detection for the distance between rods was detected using white light interferomentry (FECO) at an accuracy of $0.8 \mathrm{~nm}^{6}$. For the first time, the piezo electric transducer, which in their case is a bimorph spring, was introduced to a surface force apparatus allowing excellent control of the separation of surfaces ${ }^{6}$. The upper surfaces were attached to a fine spring with a known spring constant while the bottom surfaces were held still ${ }^{6}$. 


\section{Direct Force Measurement Apparatuses}

Beyond the use of the SFA for interactions between a sphere and a surface, it can also be used to study complex rheological properties of soft matter systems including interfaces and thin films. Villey et al. designed a dynamic mode SFA that might be used to detect a buried interface between the metal surface and the thin film coating $^{8}$. A few applications include determining the flow boundary conditions between a solid surface and fluid where the fluid is strongly absorbed on the solid surface and not removed by a contact force ${ }^{8}$. This is relevant for lubrication tissues or even electrokinetic transport properties arising in nanofluidic systems. Another application may include measuring the mechanical properties of organic and biological films supported by a rigid substrate (i.e. polymer brushes on silicon) ${ }^{8}$. Villey et al. added an in-situ absolute distance sensor that is based on capacitance measurements to determine the total distance between two metallic surfaces with a resolution of about $2 \mathrm{~nm}$ without the surfaces ever touching ${ }^{8}$. This study was just reported in 2013 so has not been widely used across the field.

\subsubsection{Atomic Force Microscopy}

Binnig et al. in 1986 published the first paper introducing the invention of the $\mathrm{AFM}^{9}$. They utilised the scanning tunnelling microscope to measure the motion of a cantilevered beam achieving forces as small as of $10 E^{-18} \mathrm{~N}$ achieving the sensitivity to directly measure forces between two single atoms for the first time ${ }^{9}$. This scientific discovery revolutionized the field of nanotechnology, surface and colloid chemistry, providing the scientist with a tool to measure forces between single atoms for a wide variety of applications from material properties to understanding biological interactions in nature. Ernst Ruska, Gerd Binning and Heinrich Rohrer were jointly awarded the Nobel Prize in Physics in 1986 for their fundamental work in the design of the first electron microscope and for the further development and design of the scanning tunnelling microscope (STM). The scientific breakthroughs recognized by the Nobel Committee in 1986 have continued to be quintessential to the measurement and understanding of surface forces and imaging at the atomic scale. 
The AFM records information from the precise movement of a mechanical probe used to scan or "feel" the surface. Piezoelectric devices facilitate accurate and precise movements of the mechanical probe across a surface. A feedback mechanism is incorporated fixing the distance between the mechanical probe and the surface inhibiting the probe coming in contact with the surface ${ }^{9}$. The mechanical probe often referred to a cantilever tip can be made of a variety of materials and in various sizes. For atomic work, the data recorded are the deflection of the mechanical probe. The AFM "cantilever spring" is a critical component of the apparatus. Where the spring must have the maximum deflection for a given force, requiring, a spring to be as "soft" as possible. Contrary, a stiff spring with a high resonant frequency is required to minimize sensitivity to vibrational noise present in the instrument surroundings ${ }^{9}$. AFM is still widely used today ${ }^{10}$ for imaging surfaces and measuring forces and allows for calibration from deflection to force.

Since the invention of the AFM there have been many modifications pushing the boundaries to achieve better resolution as well as modifications to the apparatus to provide the ability to measure a wide range samples from crystalline solids to soft matter interfaces and finally the interactions between colloidal particles.

Gunning et al. were the first to measure interactions between two deformable oil droplets in an aqueous medium using AFM. They developed an innovative method to attach a tetradecane oil droplet to an end of a silicon nitride cantilever by transferring an oil droplet from a glass slide to the tip of the cantilever. The success of the transfer of the oil droplet is dependent on the hydrophobicity of the slide and the cantilever to avoid the droplet from wetting on either of the surfaces. After the successful attachment of the oil droplet onto the cantilever, the droplet was coated in emulsifier by the addition of an aqueous emulsifier to the liquid cell of the AFM. They then were able to measure the force between the droplets in different environments by exchanging the fluid in the cell. They observed the displacement of protein, $\beta$-lactoglobulin with a small molecular surfactant, SDS as well as the effects of electrolyte screening by the addition of salt. This"proof-of-principle" paper demonstrated that it was possible to measure the force between two oil droplets in 


\section{Direct Force Measurement Apparatuses}

the size range 20 to $60 \mu \mathrm{m}^{11}$.

Soon after, Dagastine and co-workers also developed another method to measure oil droplet interactions with AFM. Instead of using a fine oil sprayed glass slide as Gunning did; Dagastine dispensed the droplet using a step-motor-controlled syringe through a $10 \mu \mathrm{m}$ capillary and studied the effect of n-decane in an aqueous SDS solution ${ }^{12}$. These two studies have demonstrated the feasibility of measuring forces between deformable droplets. However, questions have been raised regarding the role of deformation, curvature and hydrodynamics at the surfaces.

While AFM offers useful advantages when applied to measuring force/separation relationships, there are also limitations that must be accounted for. The most significant inherent disadvantage with AFM when specifically applied to soft matter interactions is that the absolute separation between bodies or interfaces is not explicitly known and must be inferred ${ }^{10}$. Although these methods have good sensitivity and can measure forces between two surfaces, they are not necessarily representative of the dynamic interactions seen in colloidal suspensions. This is due to the surface and curvature of the stationary surface not being a faithful representation of the colloidal particles dispersed in a solution.

\subsubsection{Micropippette Techniques}

Micropipette techniques were originally designed to measure the interfacial tension of deformable droplets ${ }^{13-15}$. A micropipette is a thin glass tube, usually made by the heating and drawing method whereas a capillary is a glass tube with a uniform cross section typically used for gas chromatography ${ }^{16}$. While micropipette studies began by just picking up droplets and manipulating their position by exerting a negative pressure to the micropipette they have been extended to include stretching droplets between two micropipettes under vacuum. This ease of manipulation of deformable droplets of sizes from $10 \mu \mathrm{m}$ to approximately $1 \mathrm{~mm}$ was then utilized to perform for more intricate experiments on droplet interactions. Micropipette apparatuses 
have been modified to control the separation between two distinct droplets with the addition of piezoelectric devices, as well as cantilevered capillaries.

Feng et al. used a dual-micropipette apparatus to visualise the controlled interactions between water-in-diluted bitumen emulsions during demulsification ${ }^{17}$ in the presence of ethylcellulose. Although this technique did not provide the ability to measure forces quantitatively, it allowed for some quantitative understanding to be achieved.

Most recently, Frostad et al. have developed a Cantilevered-Capillary Force Apparatus (CCFA) which combines the technologies from AFM and micropipette. CCFA has the ability measure the interactions between two droplets. The droplets are held by two capillaries, one described as the rigid capillary that is stepped towards a cantilevered capillary at a known and fixed velocity using a piezo-electric device ${ }^{16}$. The cantilevered capillary, is much like the AFM cantilever however the capillary has a much greater spring constant than a delicate AFM cantilever allowing only nano-Newton forces to be measured versus the pico-Newton or even femto-Newton forces achieved with AFM. A nano-Newton force restriction only allows CCFA to be used on large droplets on the order of 10's of $\mu \mathrm{m}^{16}$. The CCFA has a spatial resolution of hundred of nanometres; time resolution depends on the frame rate of the camera, and the technique requires individual image analysis that can be very time / resource intensive ${ }^{16}$.

However, this technique provides not only visual information on the behaviour of the droplet ${ }^{17}$ but has been also adapted to provide quantitative information on the force-distance relationship ${ }^{16,18}$. Frostad et al. utilised this technique to measure the interactions between two emulsion droplets to study the phenomena of partial coalescence $^{18}$.

\subsubsection{Optical Tweezers}

The discussion thus far has involved apparatuses that require the droplet to be physically attached to as a cantilever, surface, micropipette or a capillary. OT 


\section{Direct Force Measurement Apparatuses}

is the only direct force measurement apparatus today which requires no physical attachment to a 'surface' leading to greater flexibility of experimental set-up. Instead of physical attachment the droplet is 'trapped' by optical forces focused through an objective lens. In reviewing the work published on optical tweezers is clear that they have been used and developed for many biological applications, development for the technique for biological applications. These include biopolymer experiments focusing on understanding the mechanical properties of DNA, RNA, or the motor protein properties of myosin and kinesin

Also, a few studies have attempted to measure interactions between, for example, 1 $\mu \mathrm{m}$ spheres using optical tweezers ${ }^{19}$. The optical tweezers technique is elaborated upon in the next chapter and the focus of the bulk of the work on this thesis.

\subsubsection{Indirect Methods}

\subsubsection{Magnetic Chaining Technique}

The Magnetic Chaining Technique (MCT) developed by Calderon et al. in 1994, differs from the previous techniques described in this chapter ${ }^{20}$. The techniques discussed thus far measure interactions between two-macroscopic surfaces or between a macroscopic and a mesoscopic surface, where the droplets must be on the micrometre length scale, and mainly long-range interactions are detected ${ }^{21}$. MCT provides the ability to measure relatively short-ranged interactions between an extremely large number of ferromagnetic monodispersed droplets or particles with diameters as small as $100 \mathrm{~nm}^{21,22}$. The quantitative information obtained from MCT is an average interaction energy from an ensemble of droplets or particles with a precision as small as $10^{-13} \mathrm{~N}^{20-22}$.

The rationale regarding this technique is based on the diffraction of light by chains of magnetic particles responding to an applied magnetic field. Dilute paramagnetic droplets are aligned within a solution by a weak magnetic field causing the droplets to 
form one droplet thick linear chains parallel to the applied magnetic field. The chains of droplets are then illuminated by a white light source parallel to the droplets, and the emulsion solution emits a colour in the back-scattering direction. The emitted colour originates from Bragg diffraction that provides a direct measure of the centreto-centre distance between droplets within a chain at a precision of approximately $1.5 \mathrm{~nm}^{22}$. Due to the alignment of dipoles parallel to the magnetic field and that the repulsive force between droplets must exactly balance the attractive force the interactions between monodispersed paramagnetic droplets may be calculated ${ }^{20,22}$.

Initially, MCT was used to measure the electrostatic contribution of force between a solution of $94 \mathrm{~nm}$ paramagnetic emulsion droplets. From here others have looked at the steric contributions, the effect of the addition of polymer surfactants as well as more biologically relevant mixtures of DNA and proteins.

Philip et al. used MCT to study the stability of ferrofluid emulsion droplets in the presence and absence of polyelectrolyte to study the effect of screened electrostatic forces $^{23}$. They demonstrated that in an emulsion stabilized with an ionic surfactant (SDS) the force profile was a clean electrostatic force profile. Contrasting, a non-ionic surfactant (NP10) stabilized emulsion that is free of charge which resulted in a clean hard-sphere interaction at short inter-droplet separations. With the addition of poly acrylic acid (PAA) at concentrations below $0.01 \%$ the experiments showed reversible force curves with both electrostatic and steric contributions. At concentrations above $0.01 \%$ irreversible chaining was observed due to the binding of the polyelectrolyte that may be explained by depletion flocculation ${ }^{23}$.

Dimitrova et al. utilized MCT to determine the threshold flocculation force (the force necessary to induce irreversible flocculation), of protein stabilized emulsions. They studied three common amphiphilic proteins found in food emulsions including Bovine Serum Albumin (BSA), $\beta$-Casein (BCN) and $\beta$-lactoglobulin (BLG) at various ionic strengths from 0.01 to $10 \mathrm{mM}^{22}$. They first compared their MCT data to SFA data found in literature, and this comparison reveals a substantial difference in the force laws existing between protein-stabilized liquid droplets and mica surfaces covered by 


\section{Direct Force Measurement Apparatuses}

proteins. This difference is attributed to the conformational changes on the solid and liquid interfaces. The threshold flocculation was found to be higher for BCN than for BLG stabilized droplets. This may have been attributed to protein aggregates between approaching droplets ${ }^{22}$.

The MCT is an inexpensive method that has good precision where forces as small as 100 femto-Newtons can be measured. Another benefit of using MCT is the ability to measure interactions of droplets at the nanometre length scale whereas with other techniques such as AFM, SFA and TIRM this would not be possible. However, MCT requires a large number of monodisperse particles which rarely represents real systems. Also, most real systems are not naturally paramagnetic; it has been shown that the addition of ferrofluid to a droplet changes the deformability of the droplets.

\subsection{Motivation and Goals}

It has been described that there has yet to be an alternative method developed which can provide the flexibility that a dual-trap optical tweezer system can provide for direct force measurements. Although, interactions have been directly measured precisely in such a manner on a couple of occasions; the methods have been carried out on nearly-homogeneous system. Looking beyond to applications such as food and pharma, a method that allows for flexibility and is relativity straightforward to measure these short-ranged interactions has yet been developed.

The goal of this thesis was to develop a dual-trap optical tweezer method to directly measure interactions between micrometre colloidal particles. Ultimately to design an apparatus where interactions between less homogeneous systems, such as emulsion droplets can be directly measured as a function of separation. 


\subsection{Thesis Outline}

\subsection{Thesis Outline}

Moving forward this thesis begins with, giving an in-depth discussion of the journey taken to develop a method to measure interactions between colloidal particles (Chapter 2). Here, a brief background on optical tweezers is given, and the methodology presented including data collection and analysis providing all the steps to obtain a force separation curve.

Chapter 3 demonstrates the use of the dual trap method to measure interactions between silica beads of a known size. Here interactions between silica beads in water are presented, as well as in salt environments.

Once the interactions between silica beads were measured, the method was applied to less homogeneous systems, emulsions. Chapter 4 introduces emulsions, understanding the parameters that effect emulsion size in theory and in practice, helped with creating an emulsion with a protein interface with an ideal size and polydispersity for direct force measurement.

Finally, in Chapter 5 the dual-trap optical tweezers method was applied to sodium caseinate emulsion droplets, where interactions in a water environment were demonstrated as well as in increasing salt concentrations. Here measurements were carried out in multiple salt concentrations were measured on the same pair of droplets. 


\section{Dual-Trap Optical Tweezers}

This chapter focuses on the development of a dual-trap optical tweezers apparatus and methodology to measure interactions between pairs of colloidal particles as a function of separation. Firstly, an introduction is given on the use of optical tweezers for direct force measurement, including an overview of methods which are in current practice. Moving forward, the chapter focuses on the implementation of an improved methodology for measuring the intrinsic interparticle interactions. Here, the apparatus development, sample preparation, data collection and analysis is discussed in detail.

\subsection{Introduction and Key Literature}

The momentum of light has little effect on everyday life as the forces produced by light are too small to induce observable motion on the macroscopic world ${ }^{24}$. However, the invention of the laser allowed light to be focused into tiny volumes, providing large enough momentum to produce the motion of microscopic objects ${ }^{24}$. Phenomena with their origins in the momentum of light were first observed in the laboratory at the beginning of the twentieth century ${ }^{24}$, but the manipulation of light in order to control the microscopic world was not possible until the early 1970s. Arthur 


\subsection{Introduction and Key Literature}

Ashkin used laser light to trap and move micron-sized dielectric particles using the radiation pressure from counter-propagating lasers ${ }^{25,26}$. Sixteen years later, Ashkin et al. showed that a single beam of laser light could be used to trap dielectric particles in a stable 3D potential, and so in 1986 the single beam optical trap, dubbed 'optical tweezers' was born.

It was discovered that a single laser beam focused through a high numerical aperture lens created a focal point where a particle is trapped due to the balance between the scattering and gradient forces. The sheer number of citations of the seminal publication by Ashkin et al., 'Observation of a single-beam gradient force optical trap for dielectric particles' demonstrates the importance of this work in the subsequent continued advancement and application of the OT technique ${ }^{28}$. This paper has received more than 3,075 citations in peer review journals, and more than 1,208 of those have been in the last 5 years (Web of Science, as of 01 June 2015). Since Ashkin's discovery OTs have been extensively used in colloidal physics mainly for trapping solid dielectric spheres ${ }^{29-32}$. OT technology has also received great recognition, and in 1997 Steven Chu, William D. Phillips and Claude Choen-Tannoudji were awarded the Nobel Prize in physics for the development of methods to cool and trap atoms with laser light. Over time applications have become diverse, and through its development, particles with sizes ranging from tens of nanometres to several micrometres have been trapped, forces from femto-Newtons to nano-Newtons have been measured and time scales ranging upward of a microsecond probed in biological and colloidal systems.

Optical trapping has been particularly useful for directly measuring colloidal interactions, including screened electrostatic interactions between colloids suspended in aqueous $^{32-35}$ and non-polar environments ${ }^{36,37}$, depletion attractions ${ }^{38,39}$ interactions induced by electrical fields ${ }^{40,41}$ as well as the repulsion between particles at the oil-water interface ${ }^{42-44}$. The different optical tweezer apparatuses that have been utilised to measure these colloidal interactions mostly vary in the strategy selected for manipulating particle position and for quantifying the particle's location. 


\section{Dual-Trap Optical Tweezers}

When measuring interactions between colloidal particles the interactions are short ranged and small in magnitude (fN-pN, 1-10 nm precision required) providing a significant challenge. The methods used to probe colloidal interactions that are currently available all have limitations. These limitations may include 2D analysis of 3D-interactions, only measuring the particle position of one of the particles, low spatial resolution and even attachment of one particle to a surface or micropipette.

A large majority of OT experiments involve controlling particles at large separations from each other (greater than several microns) where laser interference on the adjoining trap is not an issue (e.g. DNA stretching experiments). However, a pair of traps at close separations $(<2 \mu \mathrm{m})$ have been shown to have an impact on each other - the trapped bead is attracted to the trap holding the other bead. Several studies have successfully measured particle interactions at close separations by removing the possibility of trap-trap interactions. These strategies include removing the second trap all together either by replacing it with a micropipette ${ }^{35,45,46}$, creating a line trap $^{39}$ or by blinking optical traps ${ }^{34,36,37}$. A more thorough explanation of each these methods is given below.

To examine interactions between a pair of polystyrene beads Gutsche et al. used a micropipette to hold one bead in a stiff optical trap $(0.085 \mathrm{pN} / \mathrm{nm})$ to hold the opposing bead. The micropipette attached to a flow cell is controlled by a pizeostage that steps the micropipette toward the optically trapped bead ${ }^{35}$. A 2D image analysis was used to extract the displacement of the optically trapped bead during the approach of the micropipette. In this study, Gutsche et al. used a stiff trap so that the exchange of fluid within the fluid cell could be achieved by using a syringe pump without fear of loosing the particles during flow. The traps used in the study are an order of magnitude larger than the traps used in this thesis the significance of this will be discussed later in Chapter 3. Gutsche et al. were able to measure the interaction forces between two colloidal particles in a range of mono-, di- and tri-valent salt solutions that were on the order of 0.3-50 $\mathrm{pN}^{35}$. This method allows for colloidal interactions to be measured when one bead is fixed. However, the question remains, how does the micropipette effect the colloidal interactions, as the particles 


\subsection{Introduction and Key Literature}

are not both free in solution?

Scanning line trap optical tweezers is a method developed where two particles are free in solution. However, both particles are located in the same trap. What makes this method possible is that the trap is elongated in one dimension to several micrometers providing a potential large enough for two particles to be trapped within the single trap. This method has been used in several studies to quantify interactions between colloidal particles ${ }^{39,47}$. This method differs from the others discussed in this section because the separations are only controlled by the optical trap. One bead is not stepped towards and away from the other. Instead, the beads move within the potential, and their separations are recorded by a camera. The probability of different separations is converted to a potential via the Boltzmann equation. One of the main difficulties with this method is that because the beads are not fixed within individual traps, they can move out of the focal plane complicating image analysis. Another disadvantage of this method is that the amount of data collected has to be significant due to the statistical game of attempting to sample rare events when the droplets experience close range interactions.

Blinking optical tweezers, as the name suggests is a dual trap approach where two traps are "blinked" out of phase with one another. The traps are alternately turned on and off by an acousto optical detector (AOD) or a beam chopper, releasing and catching the particles. This allows for the particles to be in their individual optical traps without measuring the undesired trap-trap or trap-particle interactions as only one particle is trapped at a time ${ }^{48}$. The blinking occurs at $250-500 \mathrm{~Hz}$, not allowing the particles to diffuse away from the trap. The trajectories of the particles, when released, are tracked, and the forces can be extracted if statistically enough trajectories can be measured at a given separation. Sainis et al. have published several papers using blinking optical tweezers to quantify colloidal forces ${ }^{34,36,37}$.

Finally, limited work has been done on dual trap optical tweezer methods, where two optical traps have been used to control particle positions at trap-trap separations as small as two radii. A direct force measurement would provide the intrinsic 


\section{Dual-Trap Optical Tweezers}

interparticle forces as a function of particle separation (that might differ from the data acquired for a line or blinking trap approach $)^{49}$. The challenges of using a dual trap approach will become evident throughout the rest of this thesis as this has been investigated within as a novel method.

Sugimoto et al. to our knowledge were the first to present data using the dual trap optical tweezer approach to measuring the forces between two polystyrene beads. They used a steerable mirror to translate a bead towards a trapped stationary bead. They were able to measure forces ranging from $1-6 \mathrm{pN}$ at salt concentrations of $1.151 \times 10^{-4}, 1 \times 10^{-3}, 5 \times 10^{-3} \mathrm{~mol} / \mathrm{m}^{3}$ of $\mathrm{NaClO}^{4} 49$. Although, their experimental data showed good agreement with the DLVO electrostatic interaction theory, they did not address the trap-trap interactions or trap-bead interactions that have since been recognised ${ }^{49}$.

Koehler et al. presented a comparative study examining the blinking and dual trap approaches in order to measure inter-particle forces as a function of separations. They showed that the methods did provide the same force separations as long as the calibration of the optical traps was done correctly, accounting for the interaction between traps. Koehler et al. concluded that although both approaches resulted in the same outcome, the dual trap approach had procedural advantages including reduced time of measurement and a more straightforward analysis. Most importantly Koehler et al. concluded that the dual trap approach is a robust and reliable tool to study the interactions between colloidal dispersions. However, I believe in thesis it will become evident that this method is not yet robust, and more work is required to improve our understanding of using a dual trap to measure interparticle forces.

Most recently, Nilsen-Nygaard et al. demonstrated the use of dual-trap optical tweezers to measure the interactions between emulsion droplets. This work was knowingly carried out during this thesis programme. They showed that dual-trap optical tweezers could be used to measure interactions between emulsions. Their method was applied to emulsions with various interfaces including small molecule surfactants (polysorbate 80, sodium dodecyl sulfate and citric acid ester of mono and 
diglycerides) and macro-molecular biopolymers (sugar beet pectin). Nilsen-Nygaard et al. were successful in measuring interactions where they demonstrated electrostatic repulsions between charged droplets, depletion interactions in the presence of SDS micelles and finally dynamic interactions between droplets stabilised by sugar beet pectin. Most importantly through their "proof-of-concept" study they were able to identify several key challenges with measuring the interaction between droplets. Some of these challenges include creating a "clean" emulsion to which droplet size is controlled, developing a flow cell to probe various environments, establishing methods to accurately determine droplet separation. These challenges were also found during this thesis program and will be discussed in this chapter.

In reviewing the data which have been presented in these studies by Koehler et al. and Sugimoto et al. they were both able to fit their data by the DLVO theory, however, they had few and quite noisy data points. This thesis provides an improved method where both spatial resolution and the range of the magnitude of the forces is improved reducing the noise and increasing the data that can lead to more precise understanding of interparticle forces and theory can then be more rigorously tested against experimental data. The remainder of this chapter focuses on the experimental design and optical trap apparatus.

\subsection{Optical Trapping Theory}

A single beam optical trap is produced by focusing a laser beam through a high numerical aperture lens. A dielectric particle near the focal point experiences a force due to the transfer of momentum imparted by the incident photons. This force is known as an optical force and is broken down into two components. First, the scattering force, in the direction of the light propagation and secondly the gradient force produced by the spatial light gradient in the lateral direction. When trapping of dielectric particles larger than the wavelength of laser light, geometrical or ray optics can be used to explain a stable trap ${ }^{51}$. Figure 2.1a depicts the axial trapping 


\section{Dual-Trap Optical Tweezers}

force where the light bends on entering the dielectric spherical particle that acts as a lens. The light shows zero horizontal momentum within the particle, however, after deflection the beam will have horizontal momentum. Since momentum is conserved, then for uniform incident illumination the forces cancel and there is no net horizontal component of the optical force. Figure 2.1b depicts the lateral trapping force also described as the gradient force. The gradient force arises from a dipole in an inhomogeneous electric field experiencing a force in the direction of the field $\operatorname{gradient}^{52}$.

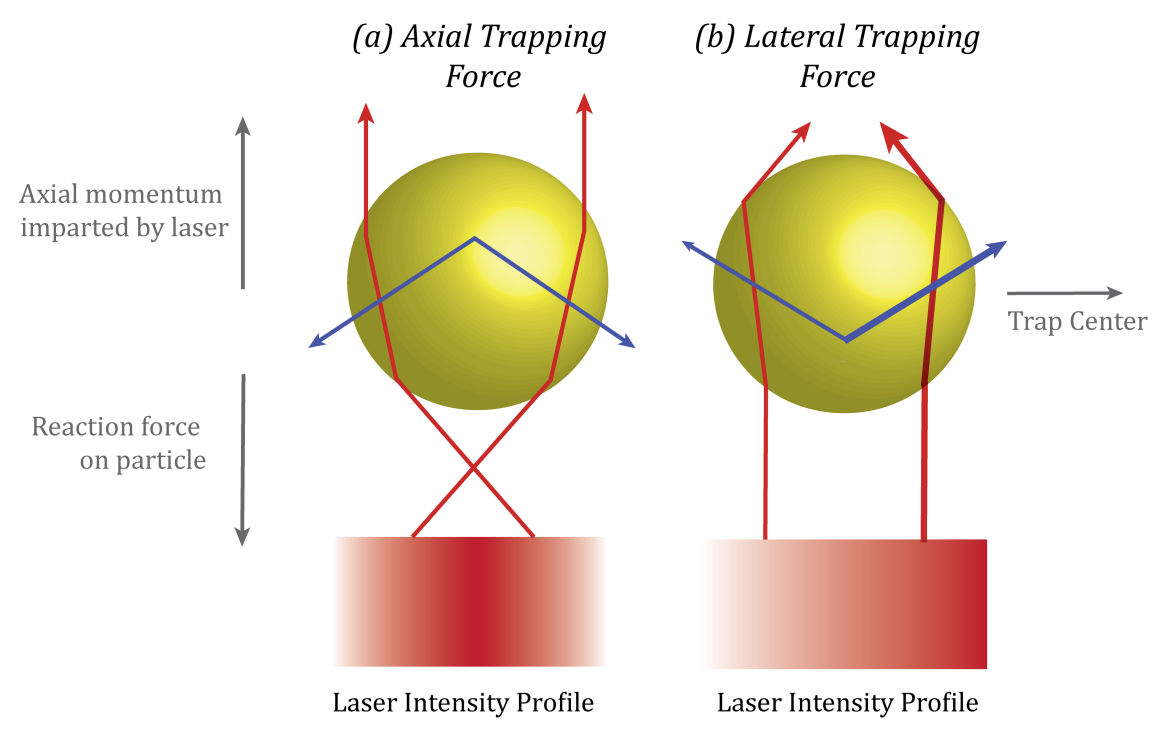

Figure 2.1.: A description of the axial and lateral trapping forces by the Ray Optics model. The red arrows depict the direction of the laser where an increased light intensity is represented in a bold line. The blue arrows represents the resulting force where an increase in the magnitude of the force is represented as a bolded line.

\subsubsection{Trapping Particulates}

Most particles and droplets of interest here meet the requirements for optical trapping, however, the ease with which a particle can be trapped and manipulated with a OT apparatus is dependent on not just the size of the particle but also the material 
Table 2.1.: A comparison of the Refractive Index of commonly studied colloidal particles in an aqueous environment used in OT experiments.

\begin{tabular}{l|c|c|c}
\hline Material & Refractive Index & Density $\left(\frac{g}{c^{3}}\right)$ & Ratio $\left(\frac{R I_{\text {bead }}}{R I_{\text {med }}}\right)$ \\
\hline Polystyrene & 1.602 & 1.050 & 1.203 \\
Latex & 1.590 & 1.055 & 1.195 \\
Silica & $1.43-1.46$ & 2.000 & $1.074-1.09$ \\
Soy Oil & 1.47 & 0.917 & 1.107 \\
\hline
\end{tabular}

properties and the laser power and wavelength.

\subsubsection{Refractive index}

Trapping requires the droplet to scatter light. Therefore, they must have a different refractive index than the surrounding medium. Most often glass or plastic particles are trapped in an aqueous medium either water or an aqueous buffer $(\mathrm{RI} \sim 1.33, \lambda$ $550 \mathrm{~nm}$ ). Table 2.1 shows a comparison of the material properties of particles that are often used for OT experiments. The refractive index for silica particles 1.45-1.6 leading to a ratio, of the refractive index of the particle to the refractive index of the aqueous medium, of approximately 1.1-1.2 provides a favourable region where the particle's interaction with light is strong enough to produce a trap of relatively high stiffness without scattering so much light that the particle will be pushed out of the trap.

In this thesis, both $1.85 \mu \mathrm{m}$ diameter silica bead particles and 2-3 $\mu$ m diameter protein coated soybean oil emulsion droplets have been predominately been used in trapping experiments. Here the oil droplets were more difficult to manipulate in optical traps due to their lower refractive index. At times during preliminary experiments it would be observed that the oil droplets would jump out of the optical trap.

The oil droplet trap instability observed was not only based on reduced refractive index mismatch but also due to the density differences. Oil droplets are less dense 


\section{Dual-Trap Optical Tweezers}

than water which causes the droplets to rise over the course of a measurement. Often the oil droplets would have to be lowered back to the middle of the well slide or channel using optical traps. This droplet creaming may be one of the causes of the droplets jumping out of the optical traps. This creaming can also affect the droplet's interactions especially if the droplet comes close to a surface. It was found by Crocker, and Grier that a long-range attraction between droplets was emphasised the closer droplets came to a surface, due to the effects of confinement of the system hydrodynamics ${ }^{53}$.

\subsubsection{Particle size}

Particle size is another critical parameter for optical trapping. Small particles exhibit greater Brownian motion than larger particles requiring greater trap stiffness. Theoretically, particles and droplets can be trapped in the sub-micrometre range using the optical tweezer apparatus. However, that is quite challenging to control. A series of known sizes of both silica and polystyrene beads have been trialled for use in the optical tweezer apparatus. Figure 2.2 shows a few images of beads and droplets ranging in diameters from $750 \mathrm{~nm}$ to approximately $4500 \mathrm{~nm}$. The $750 \mathrm{~nm}$ polystyrene beads were very unstable in the optical trap, and it was not possible to carry out an interaction measurement. Silica beads with a diameter of $1850 \mathrm{~nm}$ were found to be ideal for optical tweezer interaction measurements.

\subsection{The Apparatus}

\subsubsection{General Considerations}

Optical tweezer apparatuses can vary greatly especially in the strategy used to manipulate trap position and track particle positions. This flexibility is one of the greatest features of this technique as the apparatus can be customized for a wide 


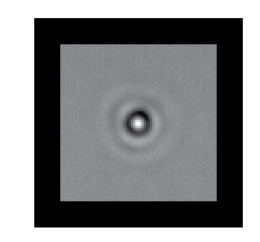

Polystyrene bead $\mathrm{D}=750 \mathrm{~nm}$

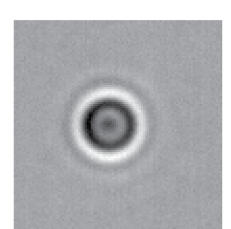

Silica bead $\mathrm{D}=1850 \mathrm{~nm}$

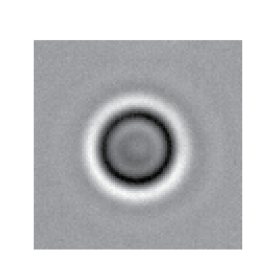

Emulsion droplet = approx $3000 \mathrm{~nm}$

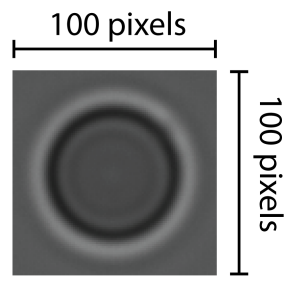

Silica bead $\mathrm{D}=4500 \mathrm{~nm}$

Figure 2.2.: Example of the images of particles and droplets traped at $1 \mathrm{~W}$ by the spatial light modulator SLM laser. The images were taken at an exposure time of $0.002 \mathrm{~s}$ and frame rate for $250 \mathrm{fps}$. The region of interest (ROI) was 100 pixels $\times 100$ pixels except for the $750 \mathrm{~nm}$ polystyrene bead that a black box was added to maintain the ROI.

variety of ways for example for single molecule and or particle experiments. The optical tweezer set-up that is used in this thesis is shown in Figure 2.3. The set-up is principally built around a standard inverted light microscope. The microscope provides two primary functions. First, it provides a means to visualise the (sub)micron particles and second, the high numerical aperture objective lens focuses laser light at the focal plane of the image to perform optical trapping. A quality high numerical aperture lens is crucial in the optical tweezer setups both for creating a high quality image and more importantly to enhance the gradient force components of the optical trap (Section 2.2).

The apparatus used in this thesis is a holographic optical tweezer (HOT) apparatus which employs a spatial light modulator (SLM) to manipulate trap positions. The SLM provides the ability to steer multiple traps and control the particle position in 3-dimensions. More discussion regarding the SLM follows later in this chapter in Section 2.4.3.1.

Data acquisition is performed by using a sCMOS (scientific complementary metaloxide semiconductor) camera that allows for high-speed photography. The common alternative to high-frequency the ability for the position of particles to be recorded and saved as an image sequence. The common alternative to high-speed video is using a quadrant photo diode (QPD) which uses a probe laser and tracks the 


\section{Dual-Trap Optical Tweezers}

interference pattern generated by the particle's movement though through the probe laser beam. The QPD output voltages that can be related to displacement by a calibration factor. The main advantage of using a QPD is that the volume of data collected for a measurement is significantly lower than for an image sequence and this allows for faster data collection (upwards of $100 \mathrm{kHz}$ vs. $1 \mathrm{kHz}$ ). However, a QPD was not used in this study, although it has capability to track the position of one particle directly, using such a detector to track two particles where probe light may be scattered by two particles at close separations can result in erroneous interference patterns. Consequently, using a sCMOS camera (faster than a CCD) was used to acquire image sequences and subsequently tracked using a precision 3D tracking program developed by Allan Raudsepp. This provided the ability to achieve the spatial resolution required for colloidal force measurements (See Appendix C) ${ }^{54}$.

In addition, the apparatus also has the capability to form a high power fixed position trap using a $5 \mathrm{~W}$ laser in addition to splitting a $2 \mathrm{~W}$ laser into multiple traps via the SLM. The $5 \mathrm{~W}$ laser could be attenuated using neutral density filters to reduce the trap power to match those produced with the SLM laser. This allowed for multiple laser configurations to be investigated as potential methods for dual trap optical tweezers measurements. This is discussed later in the chapter.

\subsubsection{Localised Heating}

A common concern in using optical tweezers to study colloidal interactions, especially with biological systems is whether the localised heating caused by the laser is significant. Several studies have looked at the effects of localised heating in optical traps, and it does exist, however, the extent of heating depends on the properties of the laser and material.

The wavelength and power of the laser impact on the degree the laser-induced heating. Experimentally, a variety of lasers have been used in optical tweezer apparatus. Some of these lasers include argon ion, helium-neon and diode laser sources ${ }^{52}$. It has 
been shown that lasers that are in the visible spectrum of light damage biological material, whereas lasers that operate in the near-infrared spectrum have less of an impact on biological materials. It is important for the laser to have minimal impact on the material allowing for a wide range of particles, droplets, cells, etc. to be probed for OT measurements. Currently, the most popular type of laser used for optical tweezer experiments on temperature sensitive biological materials is the neodymium:yttrium-aluminum-garnet (Nd:YAG) laser. The Nd:YAG laser has a wavelength of $1064 \mathrm{~nm}$ (near-infrared) ${ }^{52}$. The high powered, $5 \mathrm{~W}$ laser used in this work was similar a YD:YAG at $1030 \mathrm{~nm}$.

Studies have shown that heat absorption of optically trapped polystyrene and silica beads in both glycerol and water environments is negligible compared to the contribution from the bead's surroundings ${ }^{55}$. Peterman et al. tracked both polystyrene and silica beads in water and glycerol to determine the localised heat not only absorbed by the particle but transferred from the surrounding area including the glass slide or chamber and found that heating by the laser was not significant ${ }^{55}$. Thus, while this is an important consideration we do not expect it to be a significant issue with this work.

\subsubsection{Trap Strength}

The physical quantity that connects the observed displacement of particles from the centres of the optical traps in which they are confined with the force exerted on them is the stiffness of the optical trap. Near the focus, the potential of the optical trap is parabolic yielding a linear, Hooke's law like force-displacement relation.

The magnitude of the trap strength is dependent on several parameters including the particle, surrounding medium and laser intensity. Trap strength can be determined experimentally by several methods including the power spectral density of the particles movement, drag force measurements and examining the particle variances of the variance of the particle's Brownian motion. 
2. Dual-Trap Optical Tweezers

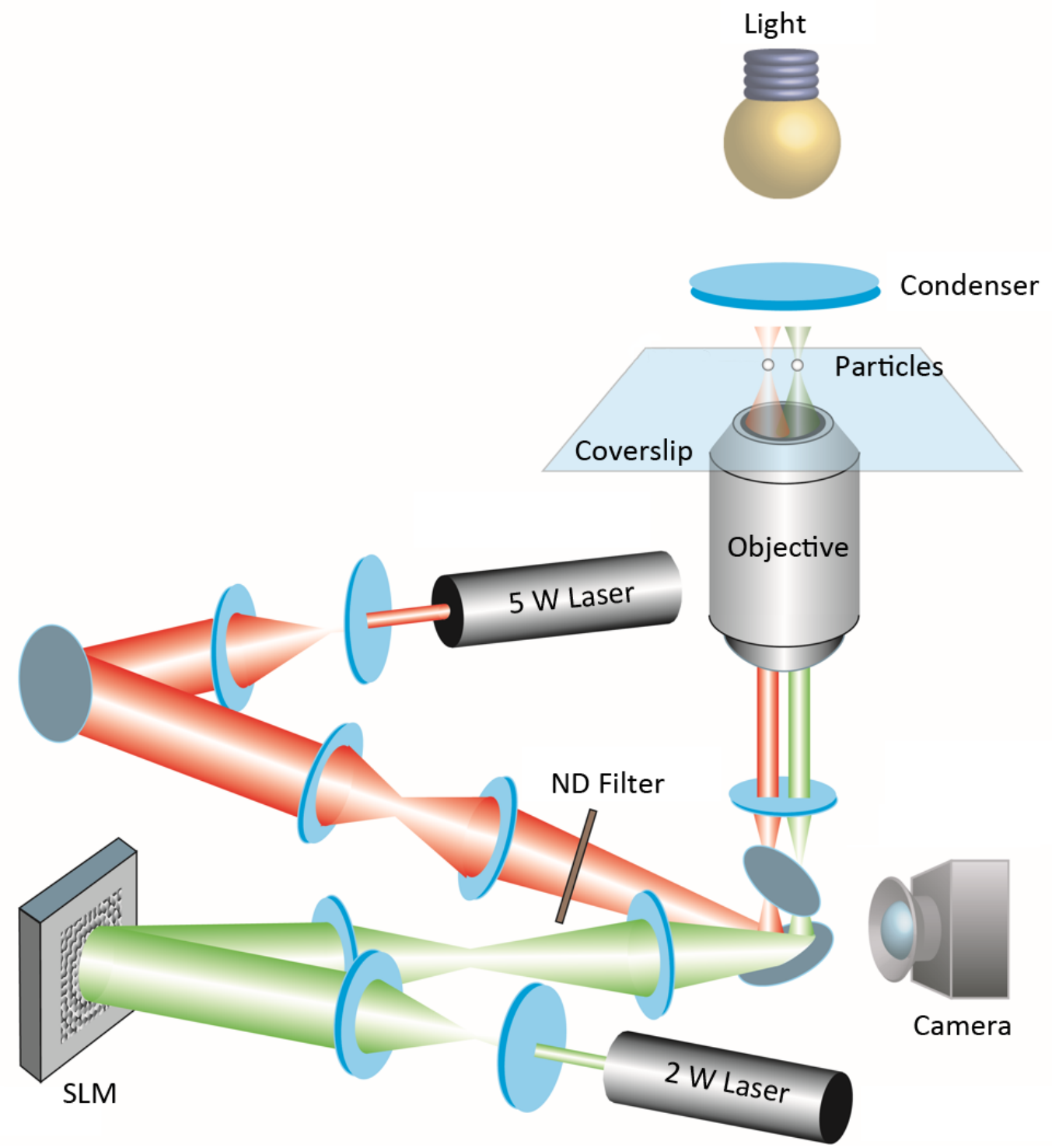

Figure 2.3.: Diagram of the HOT apparatus used in this research. 


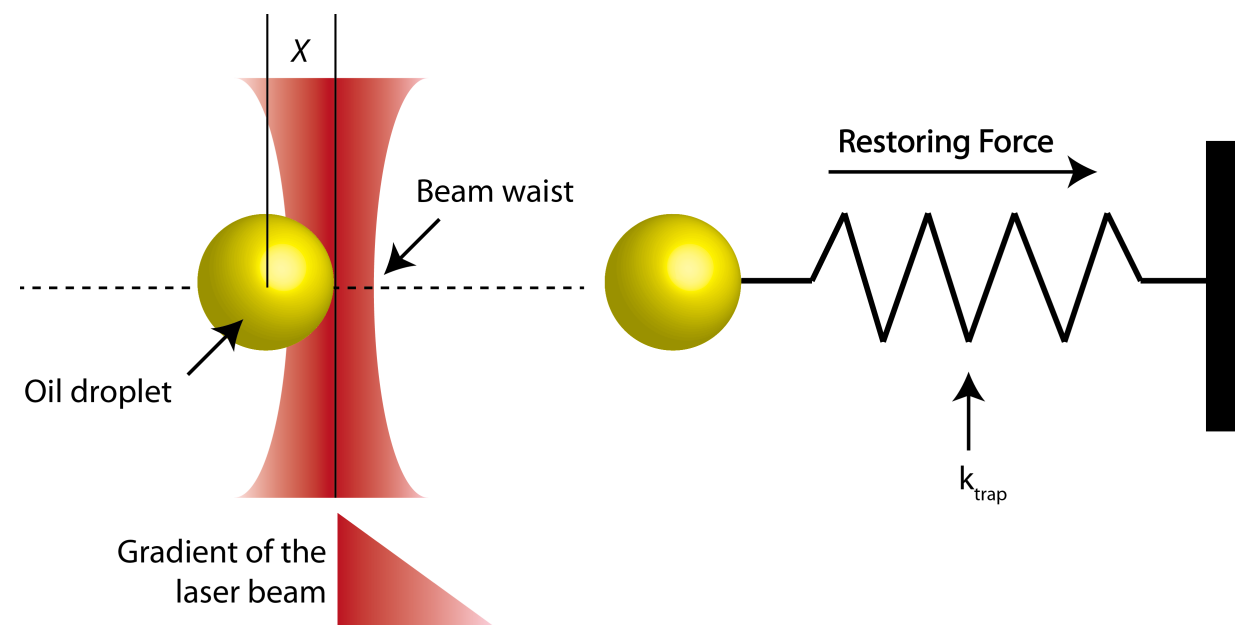

Figure 2.4.: A diagram depicting how the trap strength is related to the force. Like a spring, an optical trap has a stiffness. The force acting on a colloidal particle is proportional to the trap strength.

\subsubsection{Specific Set-up}

A schematic of the OT set-up used is presented in Figure 2.3. The optical tweezer apparatus is seated on an air table (Newport), to reduce noise from mechanical vibration. The laser tweezers (Arryx Inc.) was principally built around an inverted microscope (Nikon Eclipse TE2000-U), with a high numerical aperture lens (60x, 1.2 NA, water immersion, Nikon Plan Apo, Nikon MRD07602). The tightly focused trapping laser (Spectra-Physics) is a $2 \mathrm{~W}, \lambda=1064 \mathrm{~nm} \mathrm{Nd}$ :YAG laser, which has good pointing stability and low power fluctuations. A $1.5 \times$ auxiliary objective was used to achieve a total magnification of $90 \times$. The trapping laser is split into multiple independently steerable traps via a spatial light modulator (SLM) (Boulder Nonlinear Systems). The high-speed camera (Andor Techolology Ltd. Neo DC-152Q-C00-F1) is used to visualise and record the particles in near real-time. A second $5 \mathrm{~W}$ laser, $\lambda=1030 \mathrm{~nm}$ (IPG Photonics) was added to the optical path providing a fixed stationary trap. To reduce the laser power to achieve matching trap strengths the 5 W laser was attenuated with a neutral density filter. 


\section{Dual-Trap Optical Tweezers}

\subsection{Dual-Trap Methods}

In this section, the details of the dual trap optical tweezers method will be discussed. This method has been applied to measure interactions between both rigid spheres (discussed in Chapter 3) and protein stabilised emulsion droplets (discussed in Chapter 5). The work that is presented in this thesis is the first attempt at measuring forces in 3D using a dual trap apparatus and has provided the groundwork for additional studies of colloidal interactions in both rigid and soft materials.

The basis of this technique is to hold one particle stationary and move an opposing particle of similar charge and size towards and away from the first while quantifying the interaction. This requires controlling the movement of beads to nanometre precision, knowing the 3D position of the droplet, the size of the droplet and the stiffness of the optical traps. From these data, the forces between a pair of particles can be quantified.

The main challenges relating to the method development will be discussed in this section. This includes: strategies to control optical traps at close separations, acquiring near-real-time images for data acquisition using a high-speed camera and controlling the environment surrounding the droplets or particles using microfluidic technologies.

\subsubsection{Sample Preparation}

Sample preparation was relatively straight forward for the interaction experiments. Concentrated emulsions, or silica beads were diluted to extremely low concentrations. Emulsions are typically diluted to $0.6 \mathrm{~nL} / \mathrm{mL}$ whereas silica beads were diluted to $50 \mathrm{~nL} / \mathrm{mL}$. The samples were kept on ice until measurement to reduce microbial growth. For experiments carried out using a standard well slide $150 \mu \mathrm{L}$ of diluted sample was pipetted onto a standard well slide, and a cover-slip was used to seal the well using nail varnish. This eliminated evaporation during the experiment. 


\subsubsection{Microfluidics}

Microfluidics technology was utilised in order to control the surrounding environment of single bead/ droplet pair during an interaction measurement. As already mentioned, the goal here was to measure the interparticle forces as a function of separation in a multitude of environments. This requires a flow cell that would allow for excellent control of the fluid environment by either using flushing or diffusion. As a proof of concept, in this thesis the focus was on controlling the concentration of an electrolyte solution surrounding the beads. Many iterations of microfluidic chip designs, syringe pumps and micrometres, chip connectors and combinations thereof, had to be investigated to achieve this. At first this was thought to be a trivial task, however, after many months working with microfluidic devices it was realised that this was one of the greatest challenges of this work. It was found that in microfluidics (MFX), simplicity is imperative. It is all too easy to get carried away with in an attempt to add flexibility into the microfluidic design with additional elements. In this section, I share some of the attempts made in using MFX coupled with an OT set-up. One of the most difficult aspects of the microfluidic work was the lack of consistency.

What made this challenge unique was coupling microfluidics with optical tweezers. Achieving flow within a microfluidic chip per se was not the issue - it was providing a gentle enough flow to exchange surrounding fluid while ensuring that the beads would not be dislodged from the optical traps. Even after starting flow challenges remained: could the flow be stopped? Data acquired? Then the flow restarted? Moreover, finally, how much fluid was replaced, how did we know that we achieved a complete exchange of environments? These experiments all involved tiny amounts of liquid (or few microliters) making it extremely difficult to visualise what was happening within the channel.

The difficulty with incorporating microfluidics into our optical tweezers experimental design largely originated from our lack of trap stiffness. The optical traps in this study were weak compared to those which have been published in the literature to 


\section{Dual-Trap Optical Tweezers}

date (an advantage in increasing sensitivity). In a dual-SLM approach especially weak traps was an issue as one laser was split into two traps. An additional fixed trap approach achieved twice the trap strength as the dual-SLM approach, but it was still not stiff enough to be able to comfortably exchange fluid reproducibly without losing the beads.

\subsubsection{Flow Control}

Syringe pumps, pressure pumps, hydrostatics, and manual glass syringes and micrometers have been investigated in this research as methods to provide slow and gentle flow rate within a microfluidic channel. It was found that syringe pumps often provided irregular, pulsatile flow which dislodged the beads from the optical trap. The syringe pumps (SPS01, Labsmith) have glass inserts controlling 10s of $\mu \mathrm{L}$ of fluid, however, the pulsatile nature of the pumps caused havoc on the flow. Early on in this study syringe pumps were deemed inadequate for this task and alternatives were investigated. Moving forward, the syringe pumps were upgraded to pressure driven control pumps produced by Elveflow ${ }^{\circledR}$ : specifically the AF1 Pressure Vacuum Pump was trailed. It was found that the pressure driven pump had excellent control of both flow rate and stability achieved by the pressure versus a mechanical motor of the syringe pump. According to the manufacturer the pressure resolution and stability of $100 \mu \mathrm{bar}$, with a response time of $50 \mathrm{~ms}$ and a settling time of $100 \mathrm{~ms}$, achieving the fine control desired. Nonetheless, it was still difficult to reach zero flow, and consistently start and stop the flow without losing the beads from the traps. Even when the pump was set to a flow rate of zero, a gentle, but present flow was sometimes present.

Due to the difficulties encountered with the pressure and syringe pumps it was thought, that removing the mechanical and pressure driven devices and investigating instead gravity and capillary forces to pull liquid through the chip would be more feasible. A hydrostatics set-up, seen in Figure 2.5 was designed where a plastic disposable ten $\mathrm{mL}$ syringe was filled with a beaded solution and mounted onto a ring 
stand that was mechanically isolated from the microscope. The height was adjusted by adjusting the ring stand clamp. A capillary ran from the syringe to the channel then from the channel output to an oil reservoir. To completely isolate the channel from the fluid flow, two valves were placed on either side of the channel. These valves were fixed to a Labsmith breadboard that was mounted onto the microscope (not shown in figure). An oil reservoir was used to produce an interface, the motion of which was used to visualise the amount of fluid which was flowing. The oil reservoir was made from a $1 \mathrm{ml}$ plastic disposable pipette, where one end was sealed with epoxy.

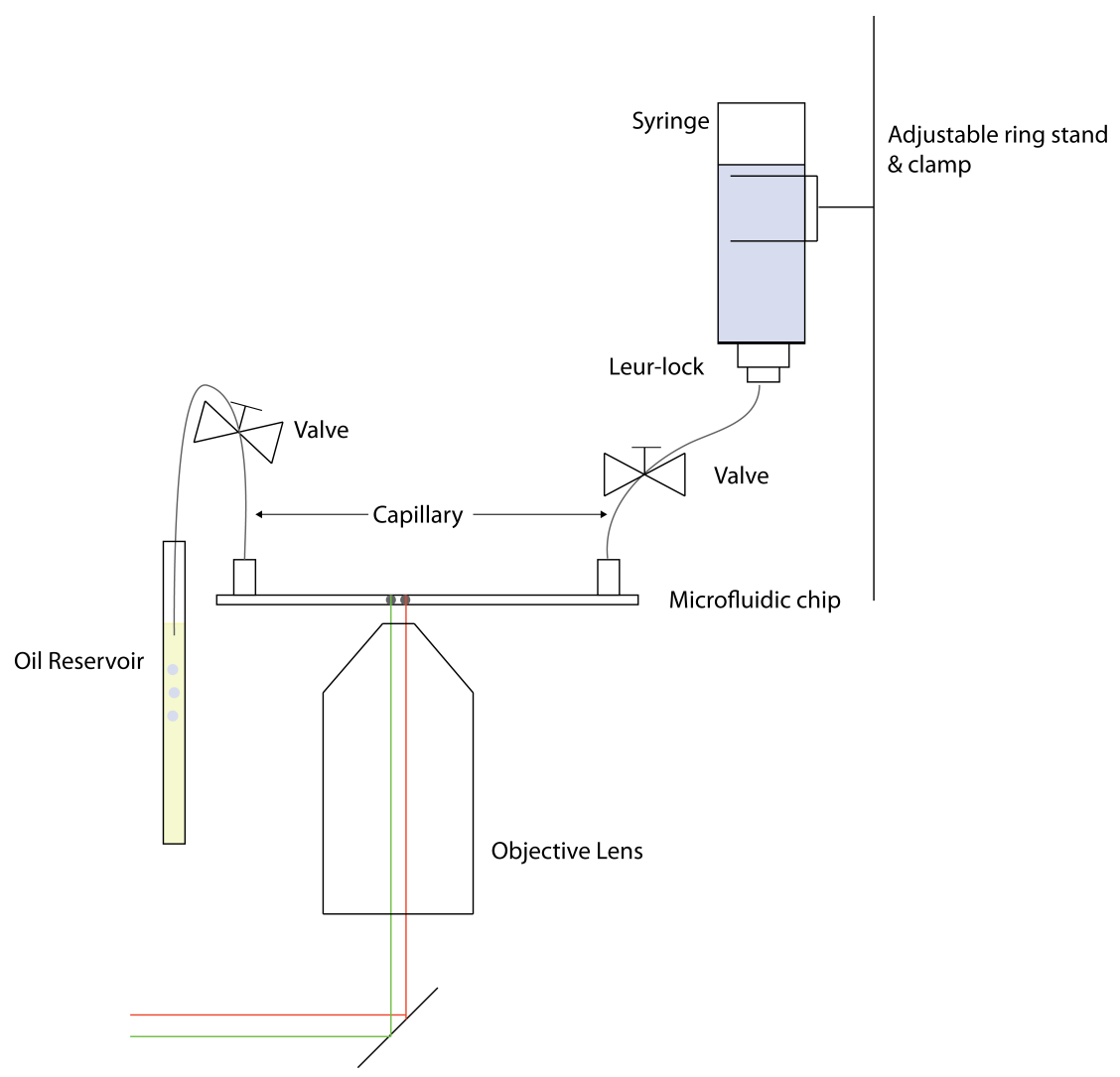

Figure 2.5.: Microfluidic set-up using hydrostatics to exchange fluid while beads were optically trapped.

Using this hydrostatics set-up proved to be the least troublesome method investigated thus far in exchanging fluid. However, a problem did become apparent where the 


\section{Dual-Trap Optical Tweezers}

valves would become blocked during the course of the experiment resulting in an incomplete measurement. The blockage was first thought to be caused by silica beads building up within the valve, but out after dissembling a blocked valve another hypothesis was posited when a lubricant to assist in turning the valve was found inside. It was then thought that the Decon90, a very basic surfactant, used to clean the apparatus before measurement could cause the lubricant to break down causing essentially the lubricant to block the valve. To dislodge the blocked valve was very time-consuming, often after several bouts in the sonicator, applying pressure with syringes and air guns, the valve could come free of debris. This blocking of the valve was not investigated further, it was decided that anything attached to the microfluidic channel that had to be touched, turned or changed during the experiment could cause the beads to be displaced from the optical traps.

The focus thus far has been a discussion on controlling microfluidic flow in order to change external environments on the fly while studying the effect on interactions. However, this was not the only issue. Even if the flow was slow enough during the exchange of fluid it was not uncommon for other beads/droplets to be sloughed off the surface, of the capillary or channel where some stuck during introduction of the sample and then get caught in the optical trap when floating by. Regularly, extraneous beads/droplets/ particles got stuck in the traps, and as they could not be differentiated by the beads of interest the measurement could not be continued.

Manual options were then explored for the exchange of fluid including using glass syringes. It was found that small syringes under $50 \mu \mathrm{L}$ were difficult to use as there was often not enough volume of liquid to clear the air bubbles from the capillary and the chip. It was found that using a $100 \mu \mathrm{L}$ glass syringe was more suited for this task. Manually exchanging the fluid using glass syringes was, however, nearly impossible because any movement of the capillary, when attached to the channel, could cause the beads to be dislodged from their optical trap.

Therefore, other methods were investigated which were completely free of any pumps or capillaries or valves and required no fluid flow. The idea would be to use diffusion 
to change the electrolyte environment.

\subsubsection{Chip Design}

Even more essential to these experiments working well was the design of the microfluidic chip. The microfluidic chips used in optical trapping experiments require specific optical properties and dimensions compatible with the working distance of the lens used. The microfluidic chip dimensions, the number of channels, dimensions of channels and the shape and configuration of the channel is vital to the success of the experiment.

There is an extensive number of microfluidic chip designs available, including companies and facilities that will manufacture custom designs. The number and configuration of channels can be customised to almost any experiment. This flexibility allows microfluidics to be used across multidisciplinary research. The microfluidic chip designs trialled are discussed in this section.
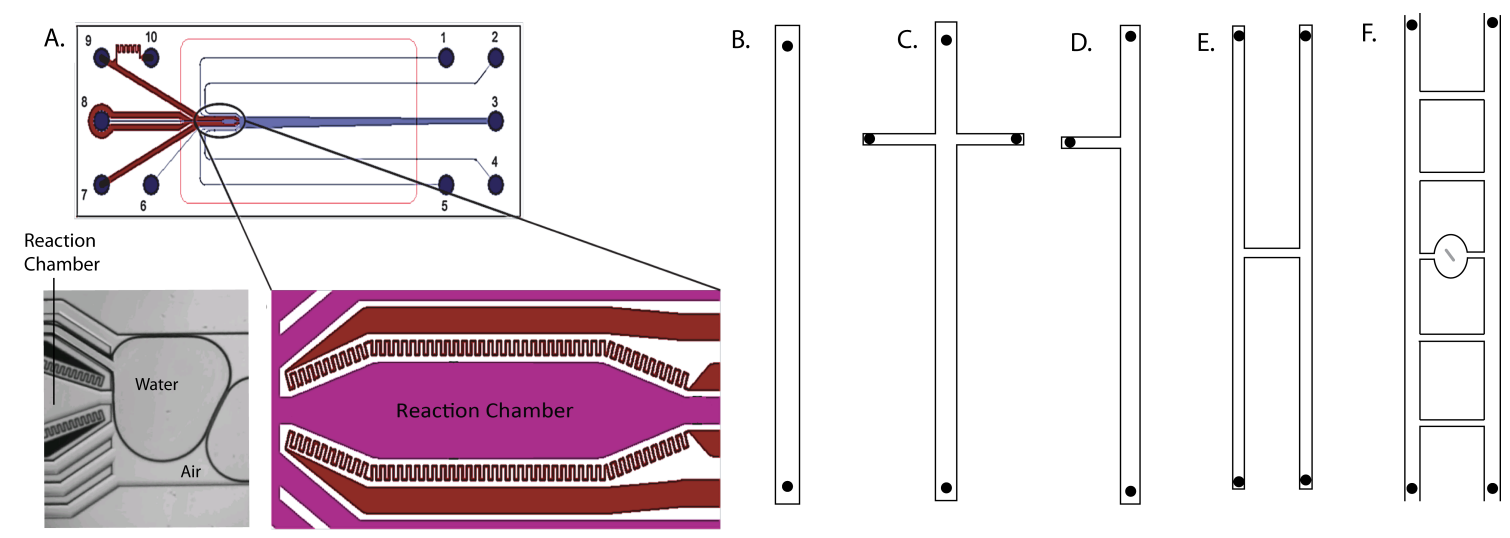

Figure 2.6.: Schematic of the optical tweezer chip designs discussed for changing the aqueous phase surrounding the beads/droplets.

Figure 2.6 A. shows the most intricate chip that was attempted for these experiments. This chip design was adapted from Uhrig et al. study. Uhrig et al. used this chip to introduce new salt environments into a reaction chamber to cross-link actin networks, 


\section{Dual-Trap Optical Tweezers}

they did not "exchange environments" but modified the environment by diffusing salt across a liquid-liquid interface which was kept separate by $\operatorname{air}^{56}$. This chip was designed and produced by Micronit Microfluidics (The Netherlands), and consisted of five channels (labelled 1-6 in Figure 2.6A.). It was originally thought that this intricately designed chip could be used to exchange multiple environments housed in each channel. This chip also had the ability to heat the reaction chamber, however, this was not utilised in this research.

This chip is extremely complicated; it is required to control air and liquid of at least 5 micro-syringes all attached to the chip via capillaries where there are at least 10 connections that can leak. This was not a novice microfluidic chip to maneuver; the biggest complication was keeping the air and water bubbles separate as well as making the connections leak proof and at the time this seemed to be nearly impossible. In hindsight, starting with a simpler chip design may have provided the know-how to be able to use this chip more successfully for optical tweezer experiments.

Due to the difficulty with the multi-channel microfluidic chip other designs were brainstormed to carry out a similar task, to provide a geometry that would allow the exchange of fluid. The designs proposed are shown in Figure 2.6 B-F. Design B. is the simplest approach of a single channel where beads/ droplets can be trapped and then a new salt solution could be flushed in and out. However, the concern here was that during the flushing, the beads could be dislodged from the optical trap (issues with this were discussed in the last section). It was thought that providing the beads a place away from the fluid exchange such as in Design $C$ and D, where there is a second channel crossing the main channel providing a place were the beads could be stored out of the flow of the main channel while a new solution is introduced could work well. Carrying on from this idea to protecting the droplets from the flow, would be to create a gradient of fluids across a cross-channel between two laminar flow streams shown in Designs E and F.

Design E, shows an H-shaped chip design. It was thought that two laminar flow streams (of different composition) could be used flowing down the long sections and 
then after some time a gradient in the cross-section between the two streams would provide a gradient of concentrations through which the beads might be moved. The droplets could be carried across the cross-channel and interaction measurements could be carried out in different concentrations of electrolyte. This brainstorming went one step further leading to Design F. Design F shows multiple cross-channels, and a chamber with a stir bar. One of the issues with working with microfluidics is the beads and droplets sticking to the glass surfaces. It was thought that if emulsion was introduced into a chamber with a stirring mechanism that the slight shearing of the sample could help prevent droplet sticking. Also, providing additional cross-channels was thought to allow for more interaction measurements in one chip.

The eventual production cost of these chip designs influenced which methods were attempted and which remained ideas for future work. Designs B and C were available off-the-shelf from Micronit; however designs D-F were not available. Unfortunately, Design C, was classified as a droplet generator chip and the channels were very small (20 $\mu \mathrm{m}$ deep and $50 \mu \mathrm{m}$ wide). The cross-section was not able to be visualised in the optical tweezer apparatus making it impossible to trapped beads in the short channel. Therefore, this resulted in the most simple chip design, a straight channel (chip B).
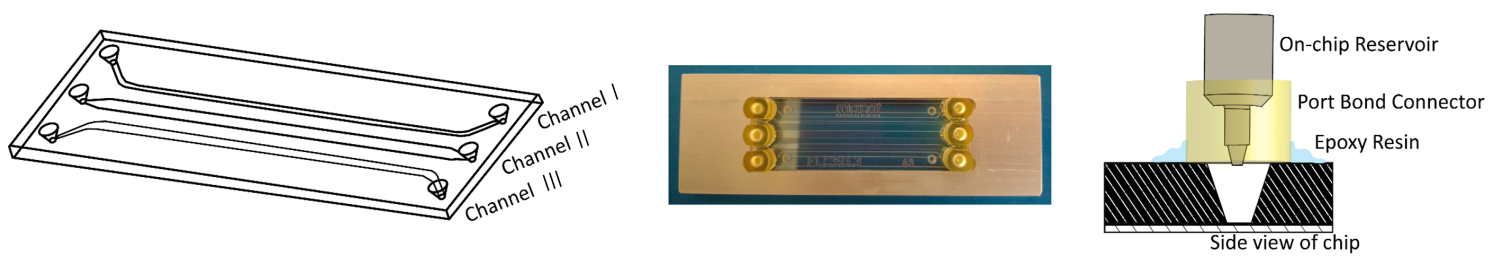

Figure 2.7.: Schematic of the flow cell used in these experiments, an actual image of the chip with the port-bond connectors attached, in a customized chip holder. A side view of the attachment of the connectors onto the chip.

A single channel microfluidic chip (flow cell, FLC50.3) was purchased from Micronit (Enschede, NL). The chip contained three single channels I, II and III: the dimensions of these channels is seen in Table 2.2. Only channel II, the largest channel, was used for these experiments. The volume of fluid in channel $I I$ is $2.8 \mu \mathrm{L}$. Slim bonded-port 


\section{Dual-Trap Optical Tweezers}

connectors (Labsmith, C360-400S) were glued onto the microfluidic chip with Quick Dry Epoxy Resin (LabSmith). Several coats of epoxy resin were applied to ensure a strong connection to the microfluidic chip and was left to dry overnight. An on-chip reservoir made of PEEK (Labsmith) threaded into the bonded port connector to provide a fluid reservoir of a volume of $85 \mu \mathrm{L}$. A one-piece fitting plug threaded into a second bonded-port connector (Labsmith, C360-101). The microfluidic chip was also glued using Epoxy resin to a custom chip holder designed (in-house), seen in Figure 2.7.

Table 2.2.: Dimensions of channel $I, I /$ and $/ I /$ specified by the manufacturer

\begin{tabular}{c|c|c|c}
\hline Channel & Width $(\mathrm{mm})$ & Length $(\mathrm{mm})$ & Volume $(\mu L)$ \\
\hline$I$ & 0.5 & 42 & 1.0 \\
$I I$ & 1.5 & 40 & 2.8 \\
$I I I$ & 1.0 & 42 & 1.9 \\
\hline
\end{tabular}

\subsubsection{Chip Connectors, Capillaries and Holders}

The way in which capillaries were connected to the microfluidic chip was crucial to the success of the experiments. The attachment needed to be both liquid and air tight, withstand some pressure during flow, and be able to be removed since the cost of the microfluidic chips this design needed to be able to be cleaned and re-used. At first, the recommended Micronit Microfluidics connections were used. These connections involved small ferrules that are attached to the end of a capillary and fit into the port on the microfluidic chip. A plastic piece then screwed down onto the ferrule tightening the seal. These connectors did not adequately seal the capillary to the chip and more often than not cracked the chip. Connectors from a different microfluidic supplier were found to improve the seal between the capillary and the chip. These connectors did not require the use of the Micronit chip holder, instead LabSmith bonded-port connectors were epoxied onto the chip directly and LabSmith one-piece fittings were tightened around the capillary onto the chip. This provided a good seal, and it was found that the chips were less likely to crack. 
A couple of different kinds of the capillary for supplying fluid onto the chip were trialled for use. This included fused silica and polyether ether ketone (PEEK) with a $360 \mu \mathrm{m}$ inner diameter. The fused silica capillary often split and cracked when cut and would crumble under pressure when being tightened down onto the chip. PEEK, plastic was more suitable as it was easier to cut and all-around easier to work with.

Finally, the initial chip holders that were developed by Micronit were made of plastic and were flimsy and did not provide enough support for the chips when attaching capillaries. The lack of support caused the chips to crack around the ports. Changing the chip holder to one made from metal, designed by Allan Raudsepp and machined by the Massey University workshop provided adequate support.

\subsubsection{Diffusion Experiment}

After realising the challenges associated with creating a flow cell with the available trap strengths, it was thought that diffusion could be a viable way of changing the environmental conditions during pair-wise interaction measurements. A salt gradient was created that would provide the ability to measure the interparticle forces at increasing salt concentrations on the same pair of beads.

A salt gradient was created by injecting a known amount and concentration of salt solution into one end of a filled-channel, displacing the initial liquid. Then the channel was sealed using plugs (One-Piece Plug, C360-101, LabSmith). This provided an interface between water and a salt environment, at a known location within the channel. Initially, the idea was to simply move the particles from one side of the interface to the other. However, the challenge with this technique was identifying the interface between the starting solution and that of higher salt concentration injected latterly.

In order to estimate the location of such a water/salt solution interface in the channel, food dye was used in order to visualise the boundary. Black food dye was used at a fairly high concentration (approximately 20\%). The microfluidic chip was filled with 


\section{Dual-Trap Optical Tweezers}

water until no air bubbles present. Then a $100 \mu \mathrm{L}$ leur-lock glass syringe was filled with $100 \mu \mathrm{L}$ of diluted food dye, and a PEEK capillary was attached to the end of the syringe and the chip. It is crucial that all air be removed from the system. The syringe was then inserted into a micro-screw apparatus, which provided fine control of the volume add. The other end of the channel on the chip was open. Food dye was added until approximately mid-channel, the open port was sealed, the syringe detached and finally, the chip was totally sealed. This series of events is depicted in Figure 2.8. A grey area was marked and the amount of dye was noted. The chip, syringes and capillary were thoroughly cleaned, and then filled with the solutions to be used for an experiment. The chip was prepared in exactly the same way with the salt.

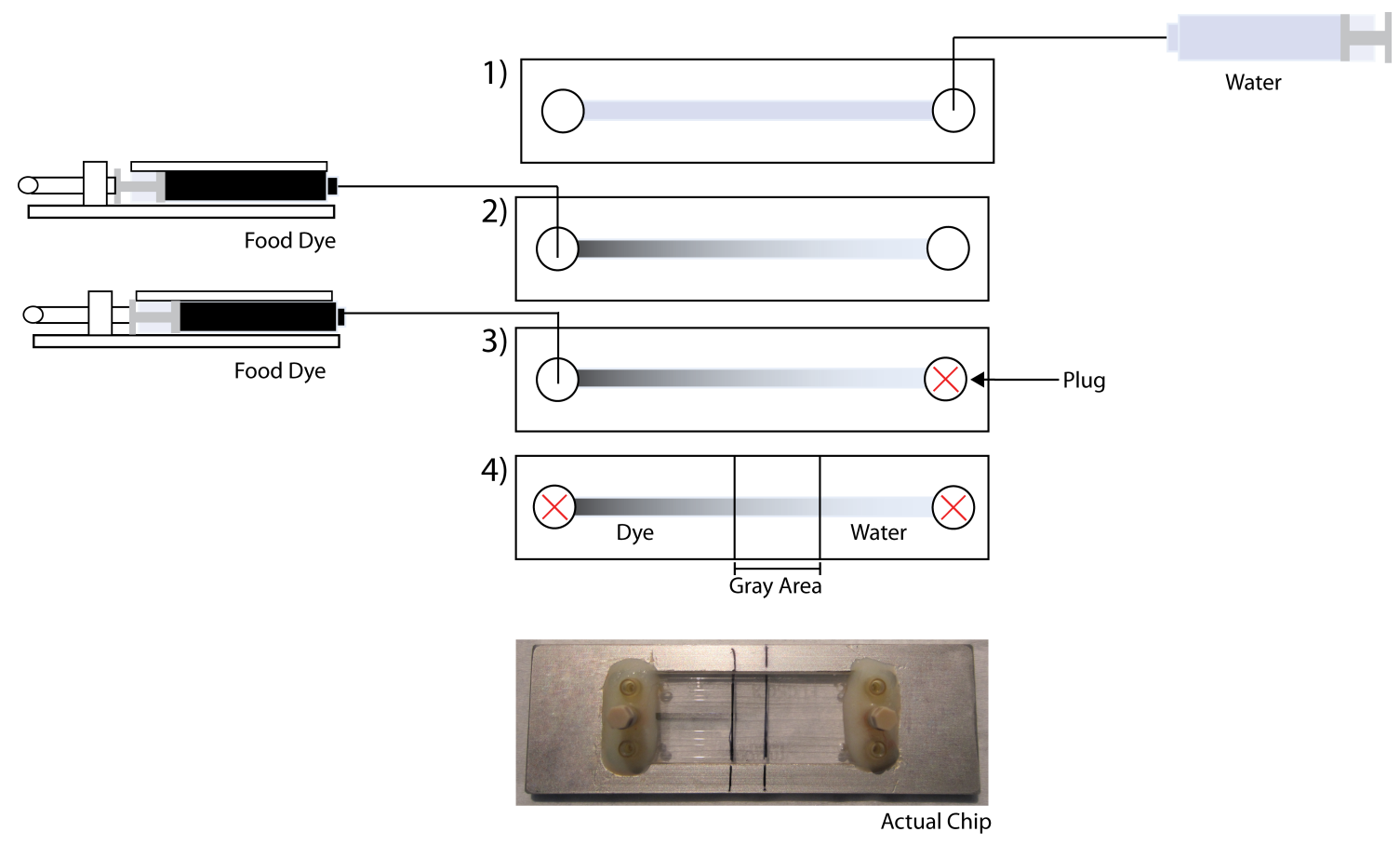

Figure 2.8.: Schematic of the injection method to create a salt gradient within a channel. (1) The chip is filled with a water and bead mixture, and the syringe is detached. (2) The food dye is added to a desired distance, the amount added is noted. (3) The open channel is sealed (4) The food dye syringe is removed than the channel sealed. The chip is labelled where the water section begins.

Separately, instead of translating the particles, the particles were held close to the 
salt-water interface and letting diffusion to slowly change the salt concentration over-time.

\subsubsection{Chip Cleaning}

It is crucial that the microfluidic chips be cleaned prior to an experiment. Several different cleaning methods have been attempted in order to remove stuck beads, bugs and other debris from the channels. Especially, with the most intricate chips used, cleaning was challenging. Several different solvents were used to clean channels including ethanol, isopropanol, acetone, and a surfactant solution Decon90. It was found that Decon90 was excellent at removing anything off the surfaces of the channels. After rinsing a channel with $10 \%$ Decon90 the channel was thoroughly rinsed with water, and no additional cleaning was required. The other solvents tested were not sufficient at removing debris from the channel.

\subsubsection{Laser Configurations}

Another challenge in developing the technique was controlling two optical traps at close separations (when the separation was less than the sum of the radii). For most of the experiment conducted thesis, the radius of two beads or droplets is on the order of 1-3 $\mu \mathrm{m}$. Two configurations of the optical traps were investigated. First, diffractive optics were used to divide the path of the laser into two controllable optical traps via the SLM. For the purposes of this chapter and thesis, this will be referred to as the dual-SLM approach. Second, an additional laser was introduced into the optical path providing a fixed attenuated optical trap, only requiring one SLM trap to be produced using holography and this will be referred to as the fixed trap approach. Before getting into the specifics regarding these approaches, it is important to understand the nuances of the SLM. 


\section{Dual-Trap Optical Tweezers}

\subsubsection{Spatial Light Modulator}

The SLM, a diffractive optical element, works by controlling the orientation of nematic liquid crystal pixels, imprinting a computer-generated phase hologram onto the SLM. The SLM can impose phase shifts up to $2 \pi$ radians for each pixel in a 512 $\times 512$ array to divert the path of the laser light. The trap position can be controlled in the $\mathrm{x}, \mathrm{y}$, and z-direction (essential to both methods). The 3D control is necessary for focusing the particles or droplets, so their image has a definite structure as seen in Figure 2.2. This image structure is necessary for the high precision particle tracking program used to determine the position of the beads in 3-dimensions. The control of the SLM traps also allows positioning of the particles/droplets with respect to the fixed attenuated trap from a second laser.

More specifically in this work, hologram coordinates were generated using Matlab. A program outputs a DAT file, a comma-delimited text file that specifies the locations, properties and number of optical traps. The output files contained the $\mathrm{x}, \mathrm{y}$, and z-coordinates, intensity and charge. Each row of data is considered a different trap. A hologram with two traps will be a matrix of $5 \times 2$ whereas a hologram to have four separate traps will have a matrix of $5 \times 4$. Table 2.3 shows an example for a 2-trap hologram. These coordinates were loaded into the software HOTUtility GUI, where the holograms are generated. Each time the coordinates are loaded into HOTUtility, holograms to be sent to the SLM are recalculated.

Table 2.3.: Example of the coordinates output for hologram generation. $\mathrm{X}$ and $\mathrm{Y}$, specify the position of the trap in the focal plan. Z, specifies the height of the trap, relative to the focal plane. Intensity, specifies the relative laser power intensities of the individual traps. Finally, charge is used to create optical vortices. Optical vortices are rings of light that can impart angular momentum to trapped objects.

\begin{tabular}{c|ccccc} 
Bead & $\mathrm{X}$ & $\mathrm{Y}$ & $\mathrm{Z}$ & Intensity & Charge \\
\hline Top & 262.900000, & 278.500000, & -25.000000, & 1.000000, & 0.000000 \\
Bottom & 262.900000, & 300.000000, & -25.000000, & 1.000000, & 0.000000
\end{tabular}

The quality of the computer generated hologram that the SLM displays determines 
the accuracy and movement of the particles during an interaction measurement. Computer generated holograms used in optical tweezer set-ups are often optimised for speed over precision. This is because the holograms must be created in real-time during measurement requiring a quick calculation and refresh rate. This optimisation jeopardizes the accuracy of trap placement and also can introduce artefacts known as 'ghost traps' in the array. Ghost traps are artefacts that form weaker traps in locations not specified in the calculation. These ghost traps reduce the laser power of the desired traps and can interfere with the measurement if the ghost traps are located in close proximity to the interaction measurement.

There is a variety of algorithms that can be used to generate the holograms based on different mathematical models. The most common algorithm used is the GerchburgSaxton algorithm (2D and 3D), others include the Roberto GS and Random Phase Mask. There is a significant amount of literature on optimising these algorithms in order to improve trap placement, reproducibility and efficiency of the programs. The algorithm used in this study was designed by a commercial company, Arryx, HOTUtility GUI. The actual specifications of the algorithm are unknown to protect the commercial interest of the company.

A short study was conducted comparing the Arryx holograms, to other known models. Red Tweezers, a LabView program written by Richard Bowman (Cambridge University, UK), provides the capability to test these other algorithms ${ }^{57}$. However, we were unable to send these holograms directly to our SLM; they had to be individually exported into another program. This caused a step that was not easily integrated into the experiment. Therefore, the actual characteristics of these algorithms on trap positions at close separations were not able to be quantified during this work. However, differences were observed. This investigation proved that the ability to precisely control traps at close separations is dependent on the hologram generating algorithm and that the Arryx holograms are not optimised for this, whereas the ability to generate, and recycle holograms using Red Tweezers showed potential for future work. 


\section{Dual-Trap Optical Tweezers}

Understanding and accounting for hologram variability in this study proved to be a challenge. Every time coordinates were loaded into HOTUtility GUI a new hologram was generated which was found to potentially have slight variations of the resulting trap positions in the $\mathrm{x}, \mathrm{y}$, and $\mathrm{z}$-directions. The difficulty was not to do with the calculation but more to do with the inability to recycle a hologram series for a particular set of experiments. It was observed when the traps were at close separation; the trap placement was much less consistent, leading to variability in the measurement. Therefore, a process referred to as trap tuning was incorporated into the method when a smooth translation of one particle towards another was required. This involved manually reloading holograms until the particles appeared correctly positioned in the focal plane and seemed to move smoothly from one step to the next. The process extended the time of the measurement especially for the dual SLM approach, described in more detail in Section 2.4.3.2. This trap tuning process also improved initial difficulties with trap jumping but did not eliminate it.

SLMs have several nuances which are important to understand. First, the trap strength and precision of the trap placement is dependent on coordinate positions. This variation may be caused by working too close to 0-order diffraction, where no matter what hologram is imparted onto the SLM stray light will be carried through the optical axis. This can be caused from flicker, reflection on the cover glass of the SLM, phase noise or other lenses and optics in the set-up. The SLM may also not be an entirely flat surface, which can cause optical aberrations resulting inconsistencies with trap placement and trap strength. This variation was accounted for by not significantly altering the trap positions from one experiment to the next.

\subsubsection{Dual SLM Approach}

The dual-SLM approach, as the name suggests, used the SLM to manipulate both beads for an interaction measurement. This method used the SLM to form four independently steerable traps. The configurations of the traps are seen in Figure 2.9 , where two traps (shown in dashed lines) are weaker, these traps will be referred 
to as holding traps. Holding traps allow for the beads to be held to the side while a measurement is recorded. This provided the ability for a measurement between a bead and an empty trap to be recorded without losing the beads. The empty trap measurement was crucial to remove the effect of a trap near a trapped bead. Moving forward, the green box depicted in Figure 2.9 1, 3 and 5, represents the recorded region of interest for an interaction measurement. This was kept constant for all interaction measurements. When the beads were translated, only two traps were used to increase trap stiffness (the total laser power is split between all traps) to ensure the beads would remain trapped during translation. Figure 2.95 and 6 , shows the division of laser intensity between two traps for the collection of reference images and the mean-squared displacement data (required to size particles with unknown dimensions). For the collection of reference images (required for the particle tracking in the $z$-direction) the bead's Brownian motion needs to be suppressed, for this the laser intensity was increased by 1.5 times (resulting in the other trap having an intensity of 0.5 ). On the other hand, for the mean squared displacement measurement the intensity of the light was required to be as weak as possible while still maintaining a trapped bead in order to maximise sensitivity. The region of interest for both these measurements was kept at $100 \times 100$ pixels. Where the blue box represents the reference image sequence, and the red box shows the weaker trap for the mean-squared displacement measurement.

The interaction step sequence, presented in Figure 2.10, shows 247 steps. Timing steps, where the top and bottom beads were stepped to the side by one pixel, prompted the beginning of the measurement. The beads were then stepped by coarse steps for the first 96 steps towards and away from a stationary bead. The moving bead was then stepped towards the stationary bead incrementally decreasing the step size from 1 pixel to $1 / 25$ of a pixel in 75 steps. This latter step was repeated twice. A Matlab program was built to generate the hologram coordinates in order to perform this stepping in an importable format. The input parameter for the program was the starting position of the moving bead which should be stepped toward or away for different size beads and droplets. 


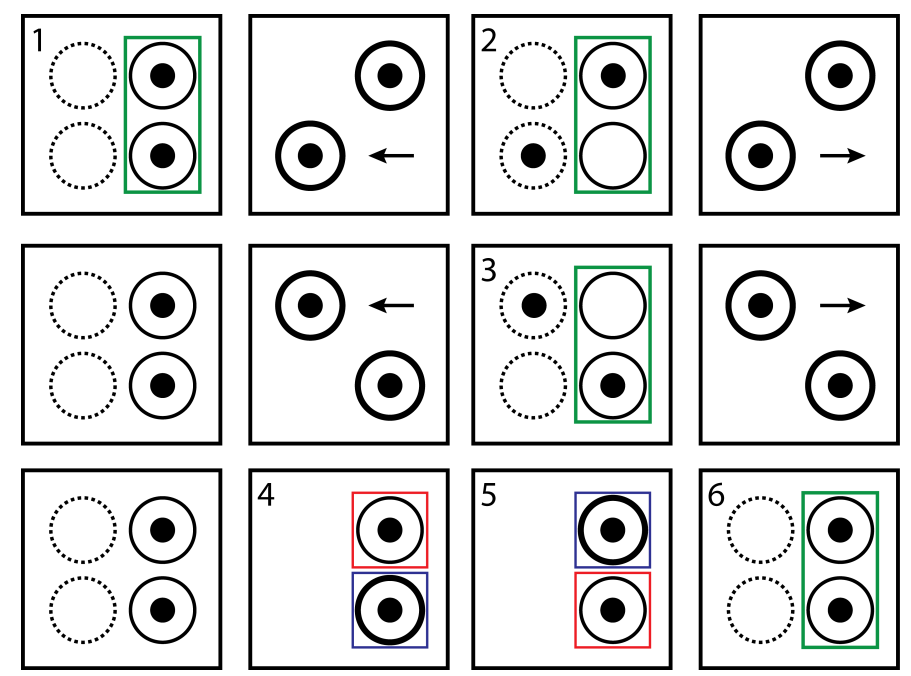

Figure 2.9.: Shows the progression of trap sequences for the dual-SLM approach. The holding traps are shown in dashed lines. The bold traps are strong traps. The region of interest for the interaction sequence is given in green, reference sequence in blue and the msd in red. The bead's translation to and from the hold traps is represented by arrows.

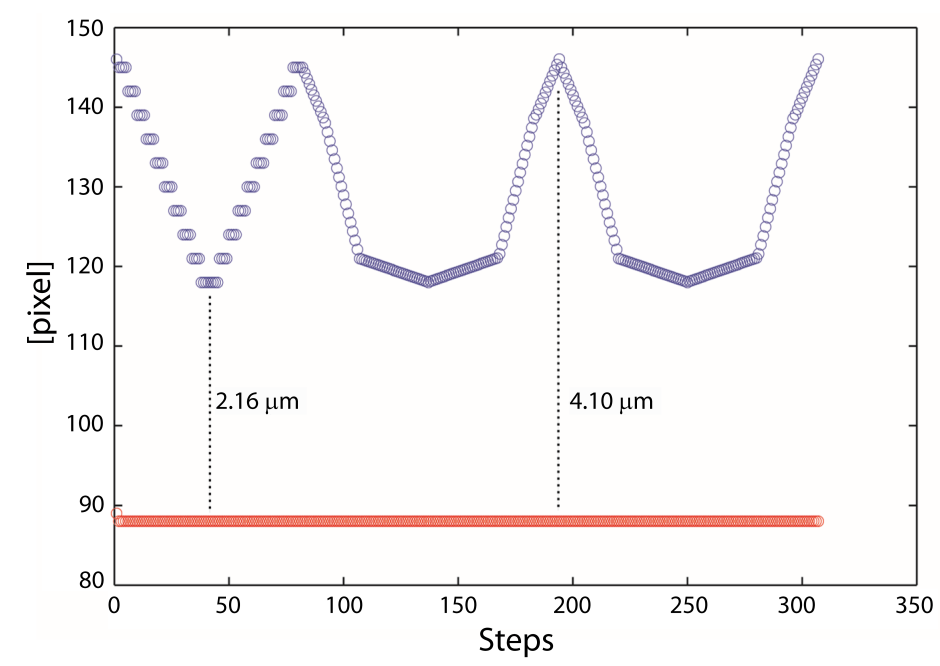

Figure 2.10.: Shows the progression of step sequences in the $y$-direction. Large evenly spaced traps were the first third of the sequence collecting $10 \mathrm{k}-20 \mathrm{k}$ frames per step, to investigative the correlations between the particle's movement. The last two thirds for the step sequence involved stepping the particle at more, smaller steps to resolve the force-displacement curve. 
The advantage of using the SLM to produce both traps for an interaction measurement is that there is an added flexibility to the measurement as both traps can be manipulated in all directions. Also, there is only one laser in the system so if there is any drift during the measurement the measurement should be less affected as both traps are created from the same light source. However, the SLM approach showed to be challenging when it comes to trap placement (particularly the requirement of particularly close traps). Figure 2.11 shows the displacement of the particles position in the y-direction, or the direction of bead approach, versus droplet separation for two emulsion droplets. A hard-sphere repulsion is clearly demonstrated at $250 \mathrm{~nm}$. However, there are only a few data points averaged over many frames not resulting in many improvements over other techniques presented in the literature. In the subset plot, the repulsion is zoomed in on, and there was an obvious chaotic movement when the traps were close. The droplets movement was consistent for each repetition, confirming it was an effect of the trap placement.

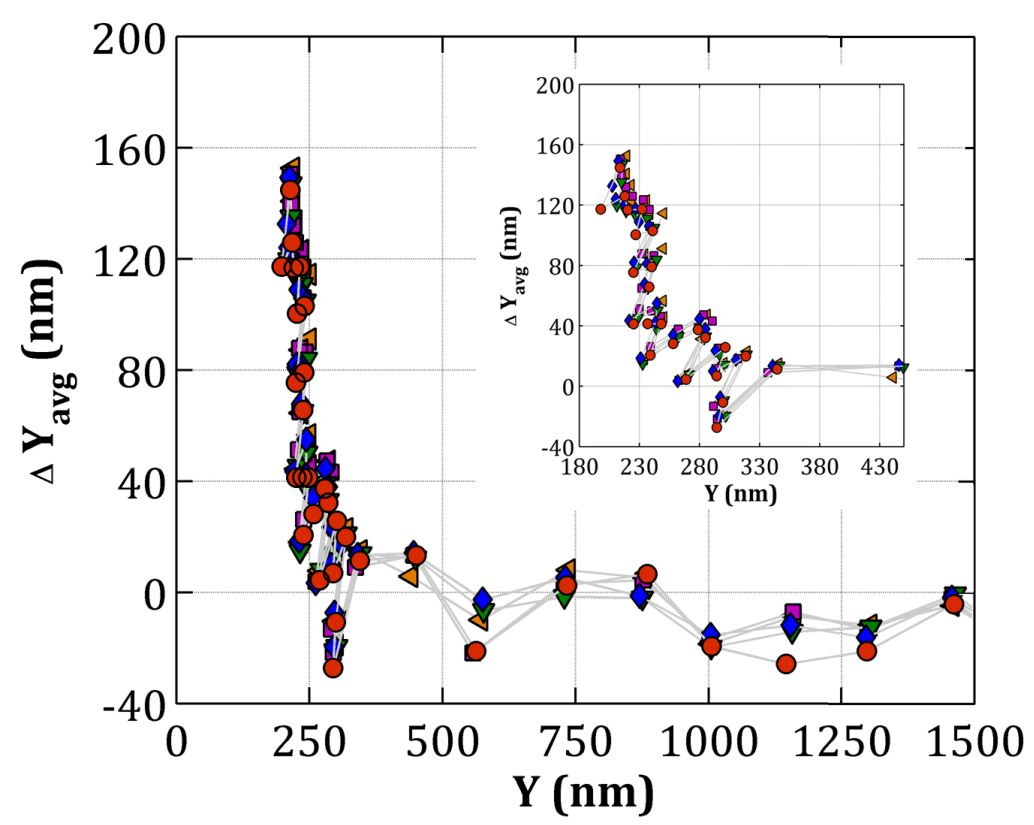

Figure 2.11.: Plot demonstrating the chaotic motion of the traps when the traps are at close separations. The different data points represent repetitions of the same set of emulsion droplets. It is shown that regardless of the pair of emulsion droplets used the same motion of the optical trap is observed. 


\section{Dual-Trap Optical Tweezers}

Logically, one may think that why not just increase the number of steps during the measurement to achieve a greater resolution? However, as mentioned the steps for this measurement were "tuned". This tuning process required the traps to be reloaded until the bead exhibited a step in the direction of approach (y-direction). This could require anywhere from 1-20 traps to be re-loaded for one step until one hologram achieved the desired position. Even though the trap tuning was only required for the steps at close separations; however, more steps would increase this tuning time. It could take several hours to tune 20 or so traps. This adds a significant amount of time to the experiment and this step in the experimental procedure was very tedious.

Also as evident in Figure 2.11 that the trap placement could be off by nearly 50 $\mathrm{nm}$ and although this is less than a pixel, it is nowhere near ideal. Due to this observation of trap placement and the inability to adjust the hologram adequately when the SLM hologram had to precisely control both particles with close traps, another laser configuration was investigated as a potential direct force apparatus method.

\subsubsection{Fixed Trap Approach}

The fixed-trap approach showed an improvement with trap placement at close separations. It was observed that the Arryx hologram generation was more accurate at calculating trap positions for a single trap than two close traps. Therefore, using a second attenuated laser to hold a stationary bead and using the SLM to move an opposing bead towards and away resulted in a smooth transition from step to step and little variation in the $z$-direction. Also, the time intensive step of trap tuning was no longer required. Finally, due to the improvement of the trap placement fewer frames were required for the measurement decreasing the time of data collection and processing. The time from trapping one set of beads to the end of the measurement for the dual-SLM approach took approximately 6 hours, whereas with the fixed trap approach about 1.5 hours was required. The remaining discussion of this chapter includes an explanation of the protocol used to measure interactions between pairs 
of silica beads (Chapter 3) and pairs of emulsion droplets (Chapter 5)

\subsection{Direct Force Measurement Data Acquisition}

Data were acquired by recording the movement of both beads during an interaction measurement during which one was stepped toward the other. Several parameters are discussed throughout this thesis relating to data collection. The relationship between the variables such as region of interest, exposure time, frame rate and camera temperature is discussed below.

\subsubsection{Camera Settings}

Region of interest Achieving a rapid frame rate coupled with a short exposure time was essential for this work in order to achieve an image sequence that captured the particle's movement in near-real time. Time resolution is dependent on the size of the window or 'region of interest' selected for measurement. The smaller the window, the better temporal resolution that can be achieved. For this work, it is important that there be sufficient space around the bead where the bead does not move out of the field of view for data processing purposes. However, if the region of interest is too big the time resolution of the measurement will decrease. Thus, the area of interest must be optimised for measurement.

Frame rate and exposure time Frame rate and exposure time are also important to measuring the interaction between beads. Exposure time is the length of time the sCMOS sensor is exposed to light, and the frame rate is the frequency of frames captured. The tracking method used herein relies on measuring the position fluctuations as fast as possible in order to obtain the most accurate description of the particle's movement. Increasing the exposure times to longer times introduces 


\section{Dual-Trap Optical Tweezers}

motion blur and reduces sensitivity, introducing statistical errors to the particle's movement. Exposure times of less than $1 \mathrm{~ms}$ and frame rates greater than $500 \mathrm{fps}$ were unable to be achieved with the required region of interest. $\mathrm{s}$

Temperature Noise suppression and image sharpness is crucial to acquire sharp images that can be successfully tracked. One of the ways that Andor achieves noise suppression is maintaining the camera temperature to $-30{ }^{\circ} \mathrm{C}$. A fan is incorporated into the camera's design in order to help keep the camera temperature low, however, the camera's fan noise can be picked up in the particle's Brownian motion resulting in an obvious fluctuation. This fluctuation was suppressed when the fan of the camera was turned off during data acquisition. However, this shortened the maximum data collection time to be about 5 minutes as the camera would increase in temperature and the fan turned back on. The thermal drift associated with this increase in temperature was removed during data analysis.

\subsubsection{Recording the Interaction Measurement}

The camera settings and trap intensities for the data required to perform interaction experiments using the fixed trap approach are outlined in Table 2.4. All interactions measurements were carried out at an exposure time $\left(t_{A}\right)$ of $2 \mathrm{~ms}$. The region of interest was chosen so that the beads or droplets were centred within the frame (where Brownian excursions would not exceed the frame). The interaction step sequence contained a timing step (stepping the bead 3 pixels in the $x$-direction), to signal when the interaction sequence begins as the camera and the SLM are not synchronized through a hardware connection. The moving bead was stepped in a triangular $y$-displacement wave with a peak-to-peak amplitude of $\Delta y \simeq 800$ $\mathrm{nm}$ (60 steps) with a period of $t=120 \mathrm{~s}$ (120 steps). Images were collected at $250 \mathrm{~Hz}$ with an exposure time of $2 \mathrm{~ms}$, producing a total measurement time of 4 minutes and approximately 60,000 frames. Each interaction sequence was completed in duplicate. Figure 2.12, shows the step sequence of the moving bead or droplet for 
this interaction measurement.

Table 2.4.: Camera settings and laser intensities for a single interaction measurement using the fixed trap approach. Background images were collected the same settings for the Interaction, Reference and MSD measurements. Explanations of the measurement terms are found in Appendix B.

\begin{tabular}{|c|c|c|c|c|c|c|}
\hline Measurement & $\begin{array}{c}R O I \\
\text { (pixel) }\end{array}$ & $\begin{array}{l}E T \\
m s\end{array}$ & $\begin{array}{l}\text { FR } \\
\text { fps }\end{array}$ & $\begin{array}{l}\text { Steps } \\
\quad \#\end{array}$ & $\begin{array}{c}\text { Frames } \\
\#\end{array}$ & $\begin{array}{c}\text { Laser Intensity } \\
\text { SLM/Fixed }\end{array}$ \\
\hline Interaction & $140 \times 100$ & 2 & 250 & 241 & 60250 & $1 \mathrm{~W} / 1 \mathrm{~W}$ \\
\hline Reference 1 & $100 \times 100$ & 2 & 250 & 280 & 70000 & $2 \mathrm{~W} / 0 \mathrm{~W}$ \\
\hline Reference 2 & $100 \times 100$ & 2 & 250 & 280 & 70000 & $2 \mathrm{~W} / 0 \mathrm{~W}$ \\
\hline MSD 1 & $100 \times 100$ & 1 & 500 & 1 & 5000 & $0.2 \mathrm{~W} / 0 \mathrm{~W}$ \\
\hline MSD 2 & $100 \times 100$ & 1 & 500 & 1 & 5000 & $0.2 \mathrm{~W} / 0 \mathrm{~W}$ \\
\hline Reference 1a & $100 \times 100$ & 1 & 500 & 280 & 140000 & $2 \mathrm{~W} / 0 \mathrm{~W}$ \\
\hline Reference $2 \mathrm{a}$ & $100 \times 100$ & 1 & 500 & 280 & 140000 & $2 \mathrm{~W} / 0 \mathrm{~W}$ \\
\hline Center of Mass & $190 \times 100$ & 2 & 250 & 1 & 2000 & $2 \mathrm{~W} / 0 \mathrm{~W}$ \\
\hline Empty Trap 1 & $140 \times 100$ & 2 & 250 & 241 & 60250 & $1 \mathrm{~W} / 1 \mathrm{~W}$ \\
\hline Empty Trap 2 & $140 \times 100$ & 2 & 250 & 241 & 60250 & $1 \mathrm{~W} / 1 \mathrm{~W}$ \\
\hline Background Images* & & & 250 & 0 & 2000 & $0 \mathrm{~W} / 0 \mathrm{~W}$ \\
\hline
\end{tabular}
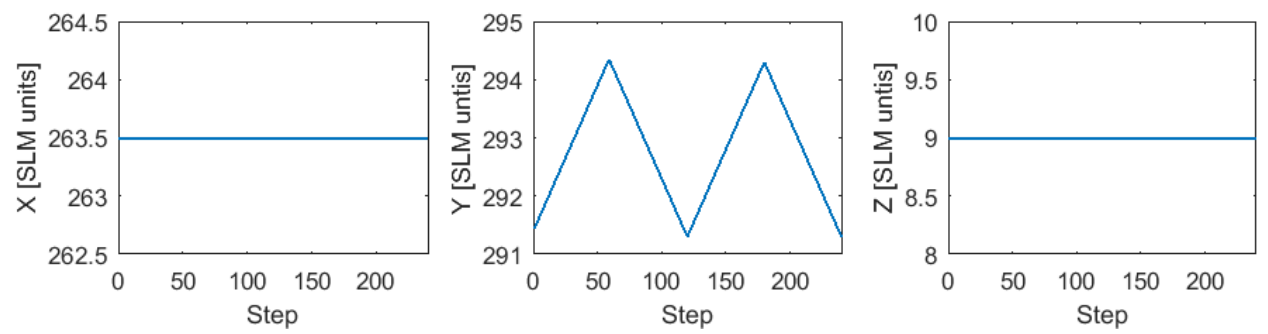

Figure 2.12.: Plot showing the trap sequence of the moving bead for an interaction measurement. The bead was stepped in the $y$-direction, where the bead was stepped 240 steps at step sizes of 0.05 SLM units.

Additionally, the interaction sequence was repeated twice, once with the moving trap empty and secondly with the stationary trap empty (refereed to as measurements Empty Trap $1 \& 2$ in Table 2.4). These measurements were subtracted from that corresponding to both traps containing beads to account for the trap-bead interaction 

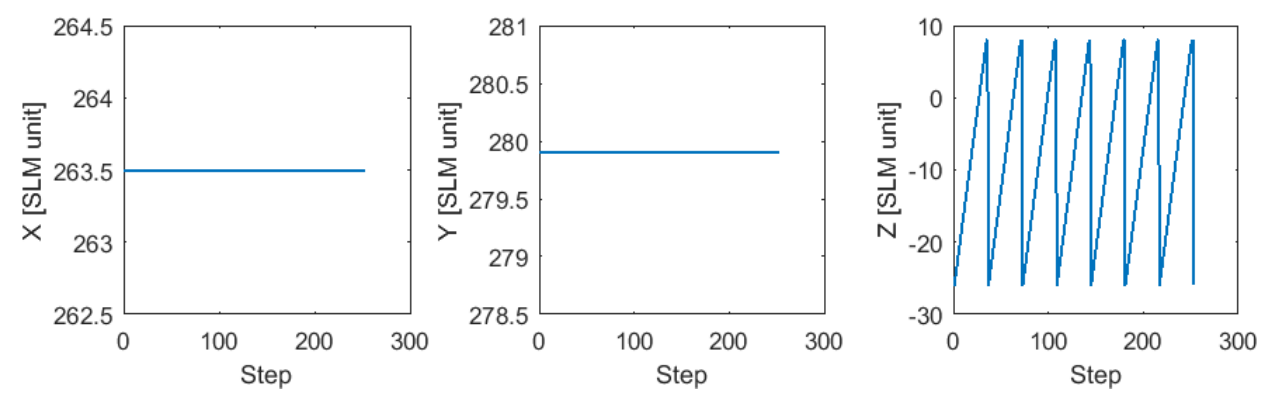

Figure 2.13.: Plot showing the reference sequence where the beads were held in the $x$ and $y$-directions and stepped in the $z$-direction in a saw-tooth manner. The bead was stepped in increments of 1 SLM unit from -26 to +8 SLM units, if the interaction measurement was recorded at $z=-9$ SLM units.

when the traps are close. This is important since the traps, when close exhibit a small attractive force on "each-other's particle".

Reference sequences were collected for each pair of beads or droplets under the same conditions of the measurement of interest. These data provide the ability for beads to be tracked in 3-dimensions and will be used for the 3D particle tracking program to determine the bead position. Figure 2.13 shows the step sequence of a reference image set. The position of the bead in the $x$ and $y$-direction was fixed and the bead stepped in the $z$-direction in a saw-tooth manner from -17 to +17 SLM units, if the tracked measurement was at $z=0$. More information regarding the tracking program and reference sequences can be found in Appendix C.

Importantly, to determine the separation between beads during the interaction measurement, an image is collected with the beads are at a large separation from each other, at a distance where the bead images have no overlap. This image will be later used to determine the centre of mass of each particle and then centre-to-centre separations during the interaction. Finally to determine the relationship between the centre-to-centre and surface-to-surface separation the size of the bead or droplet must be measured. This is completed by collecting a series of images at short exposure time where the mean squared displacement can be measured. The experimental MSD data are then fitted to a Brownian motion simulation wherein the diffusion 
constant is extracted and related to the bead or droplet radius.

Finally, for tracking purposes background images are collected for each measurement to remove shot noise.

\subsection{Data Analysis for Direct Force Measurement}

\subsubsection{D Particle Tracking}

Most commonly, centre of mass (COM) tracking algorithms are used to track a particle's position by converting an image sequence into particle positions with sub-pixel accuracy. COM tracking is considered computationally cheap and efficient algorithms have been developed. The disadvantage of this method is that the signal to noise ratio must be large. If the signal to noise ratio decreases the algorithm is no longer able to detect the particle's position resulting in erroneous results. Also, this method only tracks the particle's position in 2-dimensions. However, the forces measured between a pair of optically trap beads are in 3-dimensions. Allan Raudsepp developed a novel approach to track 3D particle position using interpolation based normalised cross-correlation which we used herein and specifics of this program are described in Appendix $\mathrm{C}^{54}$.

Correlation tracking is more computationally expensive than other methods. What makes the method expensive to run is that the position of the particle is determined by comparing an image to a kernel of successive images, i.e. reference images. The kernel, or in this case reference images are shifted in pixel increments. The position of the kernel which correlates the best determines the position of the bead. Although computationally expensive, this method has its advantages as it does not rely on radial symmetry. This is important as the droplets and beads are not perfectly radial symmetric and when two particles are close together their images overlap destroying radial symmetry. 


\section{Dual-Trap Optical Tweezers}

To track and interaction sequence, first the reference images must be processed. A reference image stack is compiled by subtracting the background, rectifying and applying a threshold to the collected reference images to remove negative values and ensure that the non-negative values do not contribute to the centroid. The images at a common step are then aligned into a step-averaged image (the images which correlate poorly are discarded and the Brownian motion and trap variation is suppressed in the $x, y$-directions $)^{54}$. The reference image stack is then cropped for each droplet to avoid the region where images overlapped at close separations ${ }^{54}$. Each reference-image stack consists of 51 z-steps.

Finally, to track an interaction measurement the background is subtracted from the interaction data in the identical fashion to the reference stack. The reference sequence is then cross-correlated to each frame in the interaction sequence. This results in the $x, y$ and $z$-positions of the particles being obtained at each step in the interaction sequence. For more in-depth explanation of this method see paper in Appendix $\mathrm{C}^{54}$.

\subsubsection{Tracked positions to force separation curves}

An example of the tracked positions of the beads during an interaction measurement is given in Figure 2.15. For simplicity, the $x$ and $y$ coordinates have been switched, the bead is travelling in the $x$-direction. When the moving bead is in close proximity to the stationary bead the stationary bead is displaced from the centre of the optical trap mainly in the direction of approach, however, there is slight movement in the $z$-direction. Also it is observed that during the measurement, the beads drifted in the $y$-direction.

First, the timing steps were removed and the data were truncated to the steps of interest, then the particle positions were smoothed over the frame rate $(250 \mathrm{~Hz})$. The tracked data were then de-drifted by fitting the positions of the beads at their furthest separation to a second order polynomial, shown in Figure 2.14, top row. The 

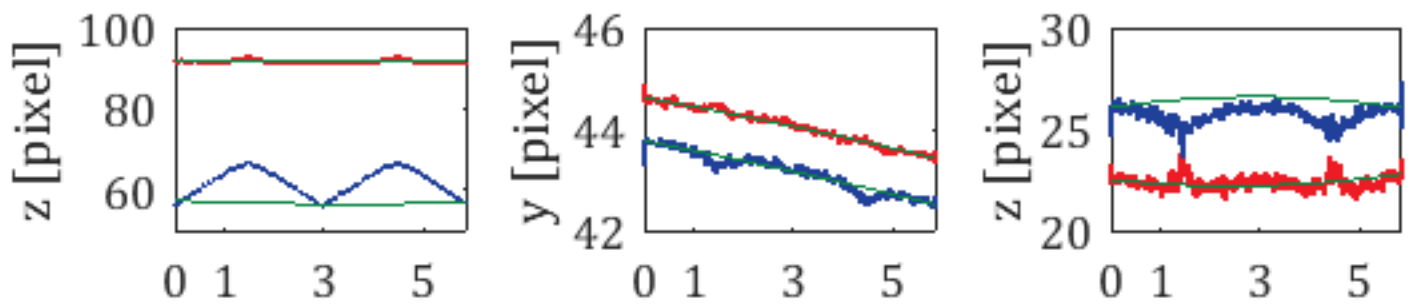

Frame $\left(\times 10^{4}\right)$

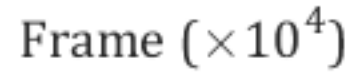

Frame $\left(\times 10^{4}\right)$
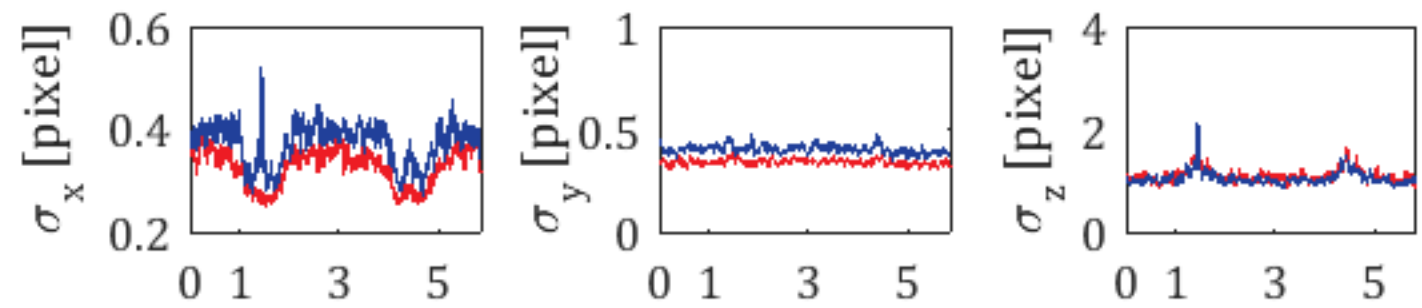

Frame $\left(\times 10^{4}\right)$

Frame $\left(\times 10^{4}\right)$

Frame $\left(\times 10^{4}\right)$
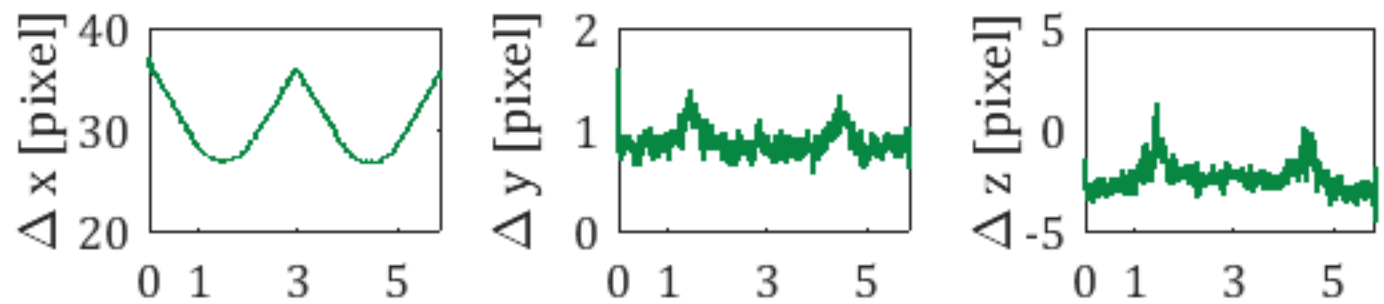

Frame $\left(\times 10^{4}\right)$

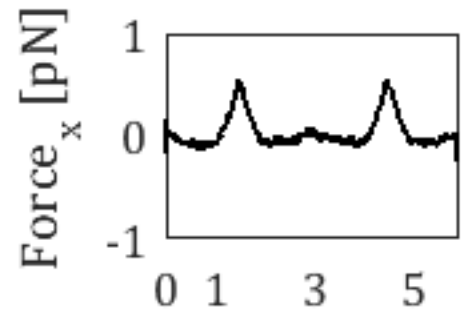

Frame $\left(\times 10^{4}\right)$

Frame $\left(\times 10^{4}\right)$
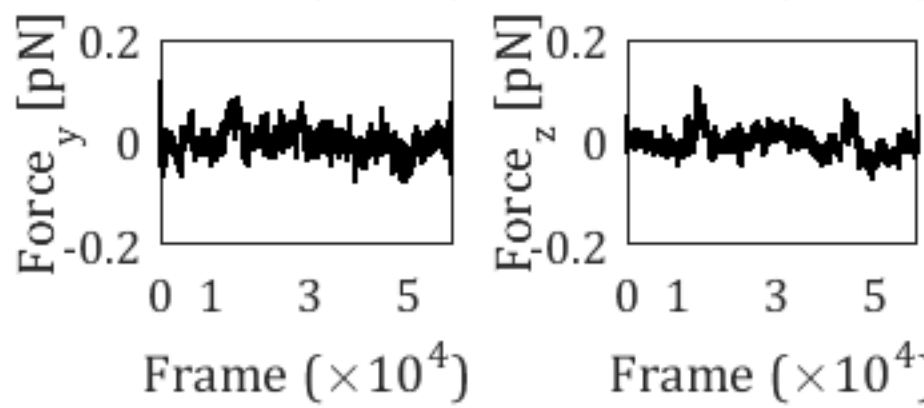

Frame $\left(\times 10^{4}\right)$

Frame $\left(\times 10^{4}\right)$

Frame $\left(\times 10^{4}\right)$

Figure 2.14.: Row 1: The particle positions of the moving bead (blue) and the stationary bead (red) are smoothed, and de-drifted. Row 2: Moving standard deviation Row 3: Bead displacement. Row 4: Force in the $x-, y$ - and $z$-directions. 

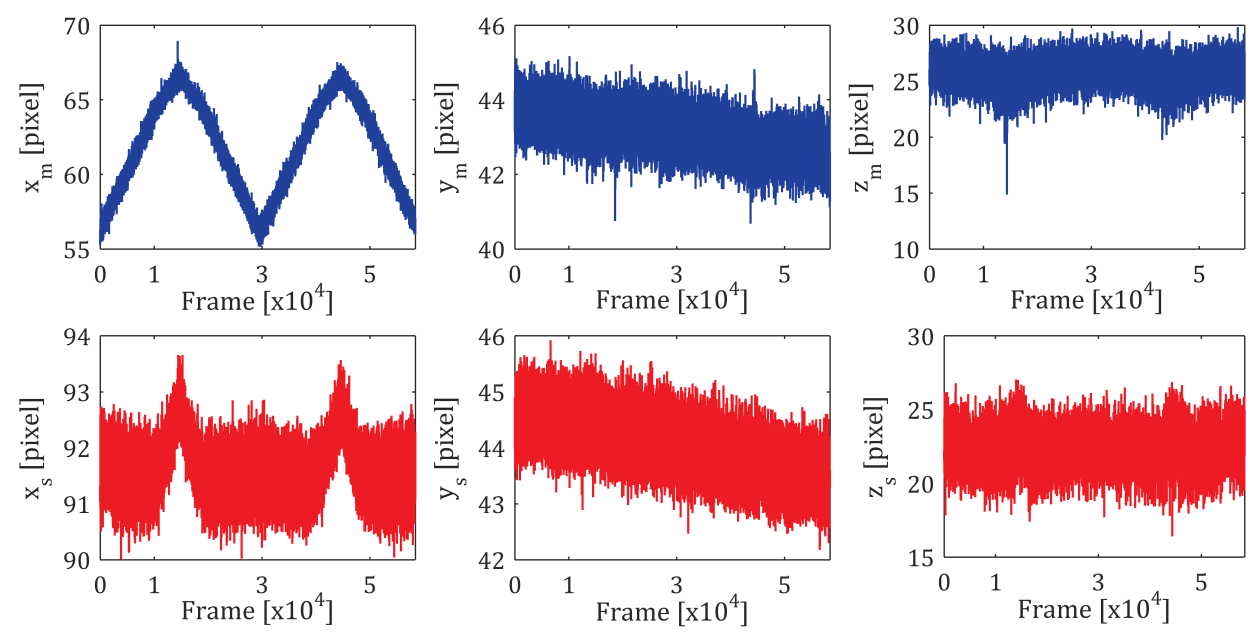

Figure 2.15.: Bead positions in the $x-, y-$ and $z$-directions of an interaction measurement. The moving bead (top row) and the stationary trap (bottom row), note the y-axis scales.

moving standard deviation was calculated, and shown in row 2 of Figure 2.14. Here, the standard deviations for the moving droplet (blue) and the stationary droplet (red) agree well. The displacement between the two particles is given in row 3 , where the flattening of $\Delta x$ when the beads are close shows interaction.

The relationship between the displacement of a bead and the force is trap strength $\left(k_{u}\right)$, (the concept of trap strength was introduced earlier in Section 2.3.1.2). In this thesis trap strength was calculated by the variance method, presented in Equation 2.1. Where $\sigma_{u}$ is the variance of a droplet's position of the raw tracked data and $u$ is $x, y$ or $z$. The variance was calculated over 2,000 frames.

$$
k_{u}=\frac{k_{b} T}{\sigma_{u}^{2}}
$$

The variance method results in a small underestimation, due to motion blur. An additional method to obtain a trap strength which accounted for a long exposure time was considered. This other method uses a Brownian motion simulation to fit the long-time mean squared displacement of a droplet in an optical trap presented. 
Further, investigation will be necessary to compare these methods to understand the extent of this underestimation.

The last row in Figure 2.14 shows the force in the $x-, y$ - and $z$-directions.

\subsubsection{Empty Trap Measurement}

During close approach in an interaction experiment the opposing trap" exerts a small attractive force on the opposing bead as discussed earlier. In order to account for this attractive force an empty trap measurement was collected. The bead was analysed in the same manner as in the interaction data. After the data were de-drifted, and smoothed, the tracked position was fitted to a cubic spline and subtracted from the interaction data. Figure 2.16 shows the empty trap data for a silica bead. The tracked positions are presented in row 1, the de-drifted and smoothed positions are shown in row 2 and row 3 shows the tracked data fitted to a cubic spline, there is good agreement between the experimental data and the fit. The data were fit instead of directly subtracting the tracked experimental data so the noise would not be amplified. 

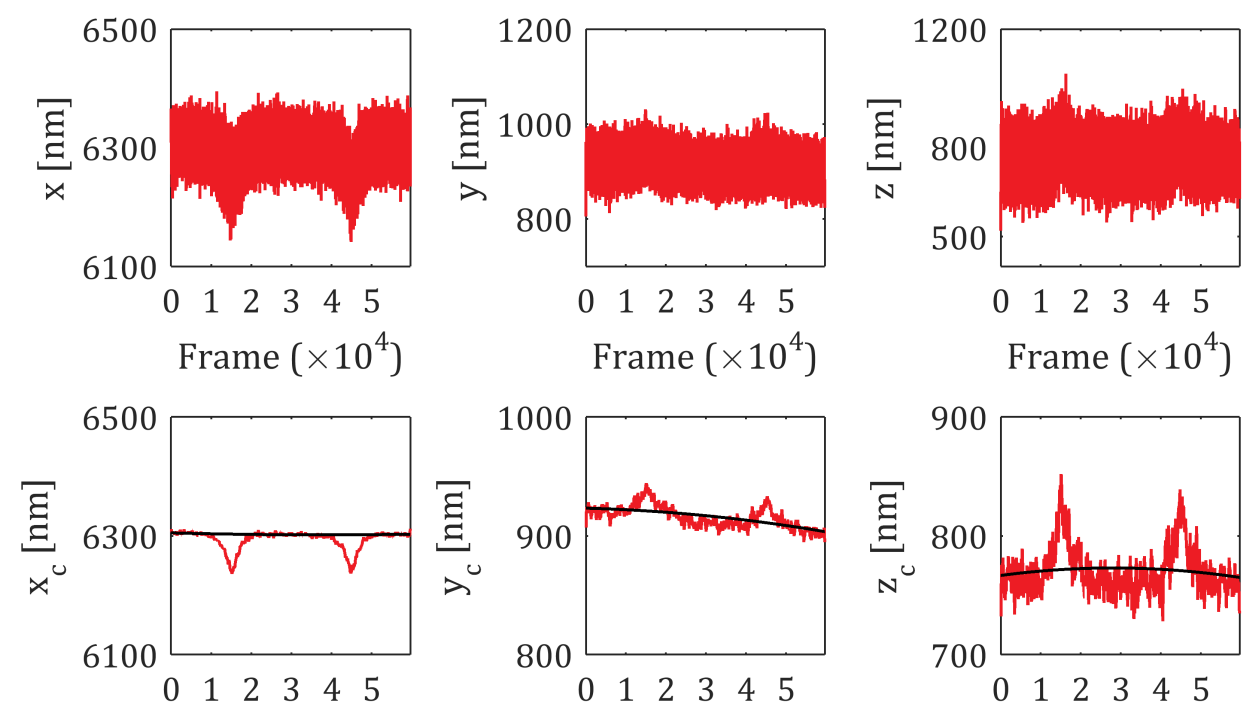

Frame $\left(\times 10^{4}\right)$
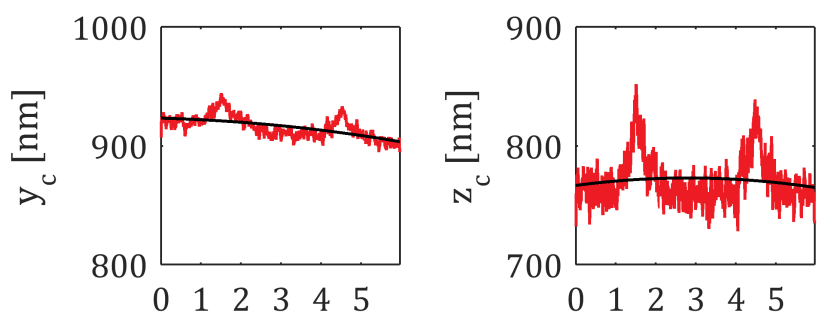

Frame $\left(\times 10^{4}\right)$

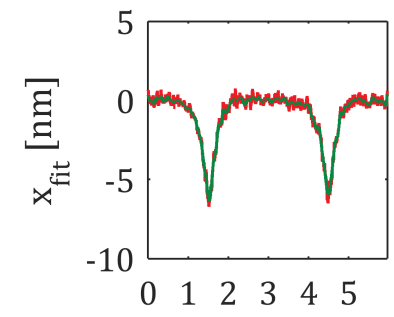

Frame $\left(\times 10^{4}\right)$
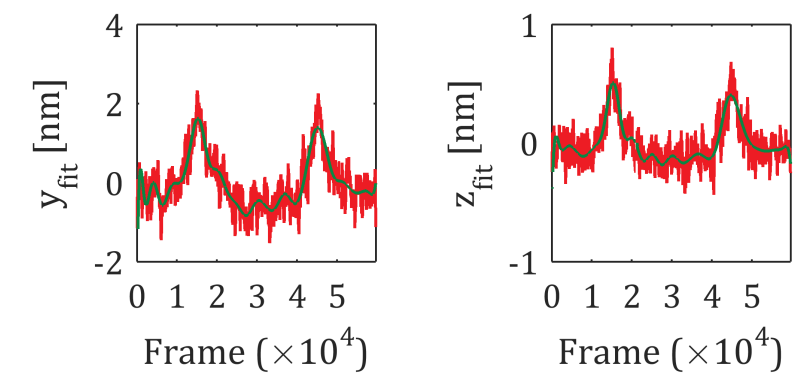

Figure 2.16.: Plot showing an example of an empty trap measurement. Row 1: tracked data of the stationary bead. Row 2: The de-drifted and smoothed positions. Row 3: The cubic spline fit of the smoothed and de-drifted data. 


\subsubsection{Bead Separation}

To obtain a force-separation plot, the absolute separation between beads must be determined. This requires knowledge of the radius of both beads and the centre-tocentre separation, and is illustrated in Figure 2.17.

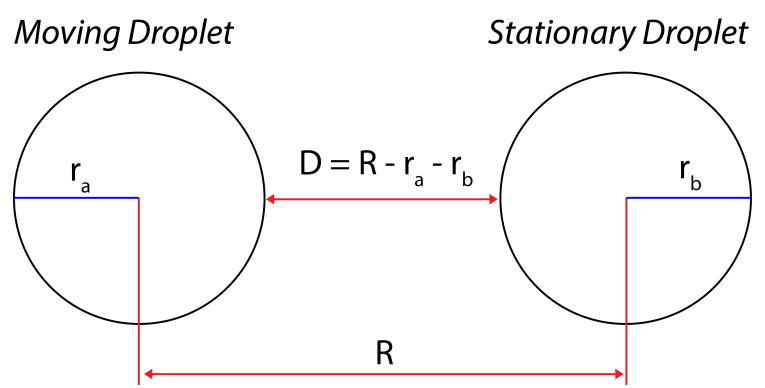

Figure 2.17.: Illustration of the bead separation.

The interactions carried out in this research involve silica beads of a known size $(a=925)$ and emulsion droplets of an unknown size. To determine the droplet sizes the short time mean squared displacement was fitted to a Brownian motion simulation explained in more detailed in Section 5.3.1, in order to extract diffusion coefficients and hence sizes.

The centre-to-centre separation between the beads was determined using the COM algorithm described previously when the beads are well separated. To determine the COM from an image of well separated beads, the background of the image was filtered, rectified, bandpassed and a threshold was applied, to remove background of the image, shown in Figure 2.18. The COM was calculated by determining the highest "energy centre" of the grayscale correlation image. The result of this is presented in Figure 2.18.

The COM image was tracked and the offsets (between recorded $x$ - and $y$ - positions of the particles COM as the origin) were determined between the tracked data and the COM calculation. These offsets were applied to the interaction data to adjust for the bead separation. 

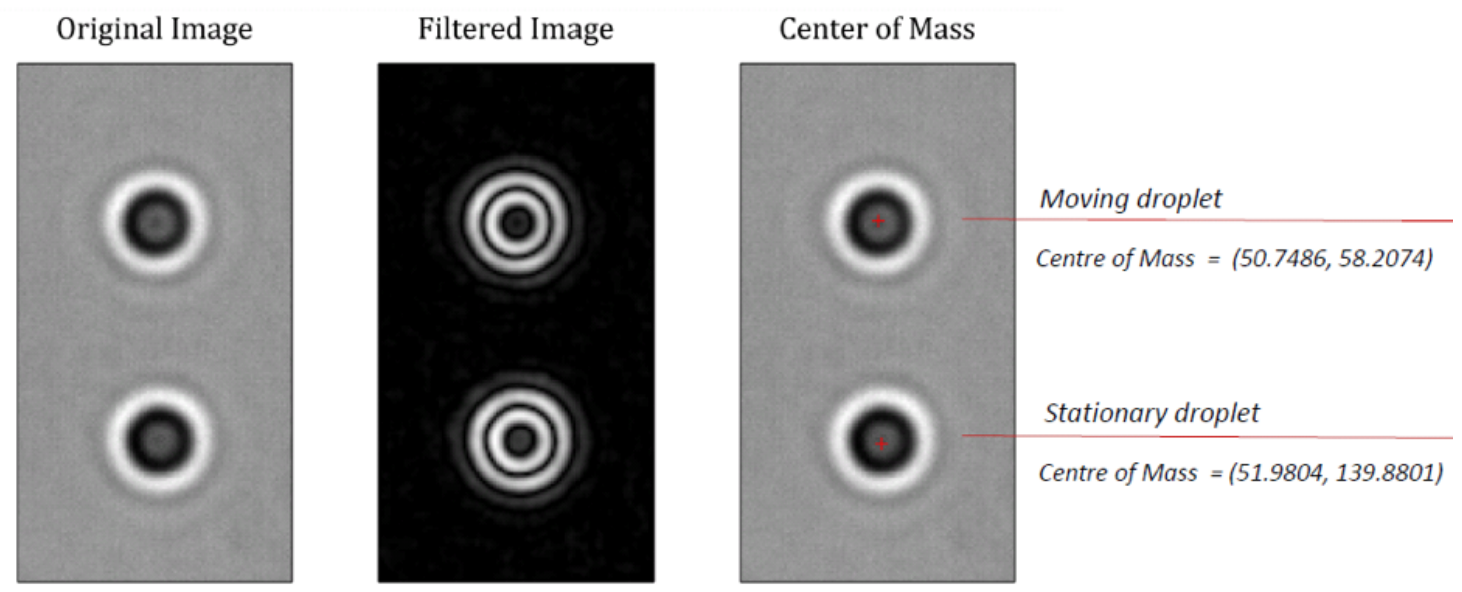

Figure 2.18.: Images of silica beads when well separated (left image). Middle image shows the result of removing the background, while maintaining the integrity of the beads image. Right images shows the calculated centre of mass.

\subsection{Summary}

The dual-trap optical tweezer method presented in this chapter provides all the details required to measure the interactions between micrometre colloidal particles. The apparatus, flow cell, data collection and processing are all described. Significant challenges have been overcome especially with developing a microfluidic experiment to measure the interactions between the same set beads in different salt environments, 3D-particle tracking and understanding our SLM nuances. Moving forward, Chapter 3 will use the fixed trap method to demonstrate this method on silica beads of a known size. Then in Chapter 5 the method will be demonstrated on emulsion droplets, a less homogeneous system. 


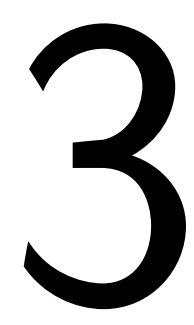

\section{Interactions between Silica Beads}

"To write it, it took three months; to conceive it three minutes; to collect the data in it all my life."

- F. Scott Fitzgerald

The information presented in this chapter demonstrates the application of the modified dual optical tweezers method introduced in Chapter 2. To demonstrate the capabilities of this method silica beads of a known size were used as a model system and their interparticle forces were directly measured as a function of bead separation; at a resolution which has never been achieved before. The interactions were first measured in a water environment. The measured force-distance curves agreed with the electrostatic component of the DLVO theory. Once the method was established, it was applied at increasing salt concentrations (decreasing Debye lengths). Interestingly, a salt concentration was found beyond which the experimental data no longer agreed with the predictions of DLVO theory. Above this salt concentration the predicted Debye length was reduced to less than the Brownian fluctuations of the particles in the traps. In this region these fluctuations dominated the apparent repulsion by restricting their particle trajectories, masking the actual nature of the electrostatic interactions. In this chapter, this observation will be expounded on while demonstrating the limitations of this optical tweezers technique. Strategies are discussed with which to mitigate this effect. 


\section{Interactions between Silica Beads}

\subsection{Introduction}

\subsubsection{Electrostatic Forces}

Electrostatic interactions often define the behaviour of colloidal suspensions and many biological processes. In foods, colloidal suspensions such as emulsions, must carry a sufficient surface charge to remain stable in solution otherwise the droplets will floc and the solution will destabilise over time. This mechanism is mainly governed by electrostatic interactions requiring understanding of the properties of the bulk phase and the droplet's interface. An example of a biological process is the ability of a cell to pack a metre of DNA into a ten $\mu \mathrm{m}$ nucleus. The DNA assembles around protein bundles, known as histone proteins; these protein-DNA bundles are solely held together by electrostatic interactions ${ }^{58}$. Providing the ability to probe and measure electrostatic interactions is of the utmost importance to further our understanding of how to control such systems that are central in food to cellular processes.

When a bead or other surface is immersed in an aqueous solution, the surfaces will become charged through ionic adsorption ${ }^{59}$. Aqueous solutions contain cations and anions that interact with partial charges on the surface ionizing the surface and creating a net surface charge. Naturally, a surface potential forms that surrounds the surface and, in this case, a bead will have a cloud of surrounding counter-ions extending into the solution known as the electric double layer. When two charged beads come into proximity and the electric double layer of both beads, overlap a repulsive force will be induced. This is due to an excess concentration of counter-ions at the double layer overlap resulting in a higher pressure between the droplet surfaces than in the bulk solution that forces the droplets to repel.

Colloidal particles can be stabilised against van der Waals driven flocculation in an aqueous environment. However, if enough salt is added to the particle's environment, the electrostatic interaction is suppressed causing the particles to aggregate. This 


\subsection{Introduction}

effect can be discussed in terms of the DLVO theory, developed by Derjagrun, Landau, Verwey and Overbeek, which is the standard theory to predict the stability of electrostatically stabilised colloidal suspensions ${ }^{2,4,35}$. The theory contains an attractive part that is attributed to the dispersion forces being strong at short distances and a longer-range screened Coulomb repulsion.

The electrostatic contribution derived from the DLVO theory is given in Equation 3.1 .

$$
F(x)=64 \pi R k T c_{b u l k} \Gamma_{o}^{2} \frac{\exp (-\kappa x)}{k}
$$

Here, $x$ is the centre-to-centre separations between two colloidal spheres of radius, $R$, $k T$ is thermal energy, $c_{b u l k}$ concentration of ions, and $\kappa^{-1}$ is the Debye length. The Debye length (in units of $\mathrm{nm}^{-1}$ ) for monovalent salts at room temperature in water is given in Equation 3.2.

$$
\kappa^{-1}=\frac{0.304}{\sqrt{c_{b u l k}}}
$$

$\Gamma_{o}^{2}$ is given by Equation 3.3 where $\epsilon_{o}$ is the permittivity of free space, $\epsilon_{r}$, is the relative permittivity.

$$
\Gamma_{o}^{2}=\frac{\sigma^{2}}{32 k T c_{b u l k} \epsilon_{o} \epsilon_{r}}
$$

and $\sigma^{2}$ is given in Equation 3.4 where $e$ is the elementary charge and $Z$ is the total charge.

$$
\sigma^{2}=\frac{e^{2} Z^{2}}{\left(4 \pi R^{2}\right)^{2}}
$$




\section{Interactions between Silica Beads}

This results in a standard first-order exponential relationship between force and distance $\left(y=A e^{B x}\right)$. The electrostatic force acting between two spherical particles can be simplified by combining expressions so that the parameters that dictate the magnitude of the force are charge, particle size and Debye length (salt concentration). Equation 3.5 shows the simplified function that was used to fit the force-displacement curves presented in this chapter.

$$
F(x)=\frac{e^{2} Z^{2}}{8 \epsilon_{o} \epsilon_{r} \pi R^{3}} \frac{\exp (-\kappa x)}{\kappa}
$$

\subsection{2. $\zeta$-potential}

$\zeta$-potential (defined further in section 4.7.2) can be determined for individual beads from knowing the surface charge $(Z)$ and the Debye length obtained from fitting the force-displacement curve. The relationship between surface charge and $\zeta$-potential is given in Equation 3.6

$$
Z=\left(e \zeta / k_{B} T\right)\left(R / \lambda_{b}\right)(1+\kappa R)
$$

The Bjerrum length, $\lambda_{B}=e^{2} / 4 \pi \epsilon \epsilon_{0} k_{B} T$, captures the polarizability of the solvent; it is the separation where two elementary charges have an electrostatic interaction energy of $k_{B} T$.

\subsection{Experimental}

The interparticle interactions between monodisperse spherical silica beads with a diameter of $2 r=1.850 \mu \mathrm{m}$ (Bangs Laboratory Inc, CV $10-15 \%$ ) were measured. The beads were diluted so that there were only a few beads per $100 \mu \mathrm{L}$. The medium 
that the beads were diluted in varied depending on the environment to be probed. Either fresh ultra-pure water with a resistivity of $18.2 \mathrm{M} \Omega \mathrm{cm}$ at $25{ }^{\circ} \mathrm{C}$ or sodium chloride at concentrations from $100 \mu \mathrm{M}-5 \mu \mathrm{M}$ (ACS reagent, Sigma-Aldrich). The dilute bead solutions were kept on ice, to inhibit bacterial growth until the slides were prepared. The interactions were imaged in water filled well-slides unless otherwise stated. Cover-slips was sealed with nail polish to eliminate evaporation as well as drift during the experiment. The same beads could be trapped and manipulated for many hours.

The details of the optical tweezers apparatus used for force measurements is outlined in Section 2.3.2. Briefly, the beads were imaged in bright-field using $60 \times$, numerical aperture 1.2, plano-apo, water immersion objective (Nikon) in combination with a $1.5 \times$ auxiliary objective for a total magnification of $90 \times$. Images of the interactions were captured using a 16-bit sCMOS camera (Andor Neo) with acquisition frame rates $f_{A}=250-500 \mathrm{~s}^{-1}$, and exposure times $t_{A}=1-2 \mathrm{~ms}$ at a background intensity of $\langle I\rangle \simeq 20 \mathrm{k}$ counts unless otherwise noted. The optical traps were formed by a $2 \mathrm{~W}$ $\lambda=1064 \mathrm{~nm}$ laser (where an SLM was used to control bead position in 3-dimensions) and a $5 \mathrm{~W}, \lambda=1030 \mathrm{~nm}$ laser, which formed a fixed trap. Holograms for SLM control were generated using the software HOTUtility, a software supplied by Arryx.

All analysis was performed using Matlab (Mathworks). The position of each bead was determined using a particle tracking algorithm using an interpolation based normalized cross-correlation approach developed by Postdoctoral fellow Allan Raudsepp. The details of this method can be found in the manuscript titled "Developing a video tracking method to study interactions between close pairs of optically trapped particles in three dimensions " 54 a draft of the submitted paper is found in Appendix C. 


\section{Interactions between Silica Beads}

\subsection{Results}

\subsubsection{Interactions Between Silica Beads in Water}

The electrostatic interactions between two silica beads were measured in water. A bead was stepped towards and away from a trapped stationary bead. Figure 3.3 shows a progression of images during such an interaction. Here, it can be seen that bead $B$ is stepped toward bead $A$ until bead $A$ is displaced from the centre of the trap by approximately 1-3 pixels. When bead $B$ is retracted from bead $A$, bead $A$ returns to the centre of the optical trap. This displacement is proportional to the force exerted between the two beads. The starting separation of the beads was chosen so that when the beads are close there is minimal z-displacement, this allows for the dominant force to be in the direction of the bead approach.

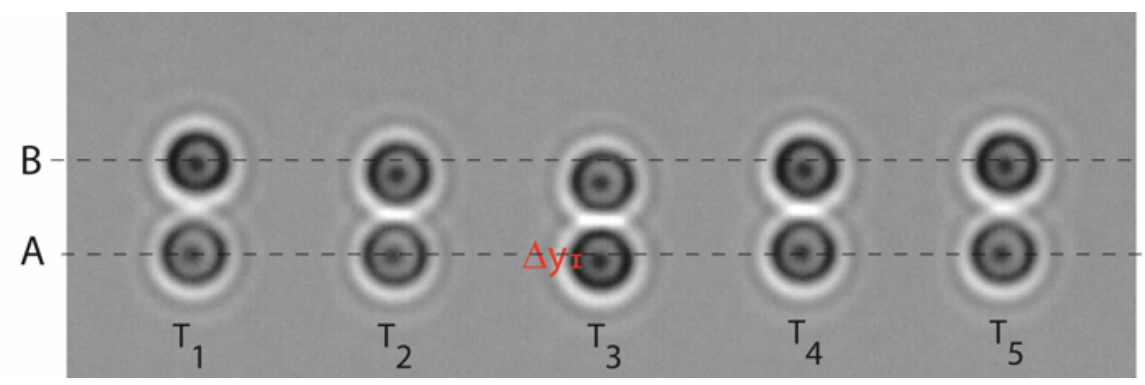

Figure 3.1.: Visualisation of the interaction between two silica beads in water in a well slide. Bead $B$ is stepped toward bead $A$ between $T_{1}$ and $T_{3}$, then stepped away $T_{3}$ and $T_{5}$. Upon bead $B$ approaching bead $A$ the beads did show some z-movement.

More specifically, the moving bead was stepped in a triangular $y$-displacement wave with a peak-to-peak amplitude of $\Delta y \simeq 800 \mathrm{~nm}$ (60 steps) with a period of $t=120$ s (120 steps). Each interaction sequence was completed in duplicate. Images were collected at $250 \mathrm{~Hz}$ with an exposure time of $2 \mathrm{~ms}$, producing a total measurement time of 4 minutes and approximately 60,000 frames. Additionally, the same trap sequence was repeated twice, once with the moving trap empty and secondly with the stationary trap empty. These measurements were subtracted from their corresponding 
bead-bead interaction to account for the trap-bead interaction when the traps are close (when the beads are at close separation the opposing trap exhibited a small attractive force on the bead held in the opposite trap that is accounted for by the empty trap measurement). The image size for the interaction measurement was 120 $\times 100$ pixels. The laser power for the fixed trap $(\lambda=1030 \mathrm{~nm})$ and the moving trap $(\lambda=1064 \mathrm{~nm})$ were fixed at $1 \mathrm{~W}$. The bead size was assumed to be $2 r_{A}=2 r_{B}=1.85$ $\mu \mathrm{m}$ based on the manufacturer specifications.

The position of both beads was determined by 3D-tracking. The bead position was compared to a series of kernel images. The kernel images (referred to as reference images) were collected at $51 z$-positions, $z=-25$ SLM units to $z=+25$ SLM units, where the interaction measurement was recorded nominally at $z=0$ SLM units. Reference images were collected with an image size of $80 \times 80 \times 51$ pixels. The overlap of the beads image when at close separation was excluded by cropping the reference image sequence to $61 \times 31 \times 51$ pixels for 3 D-particle tracking.

The tracked particle positions were corrected for low-frequency drift by fitting the beads movement when at their furthest separation from a line. The drift is due to camera warming during the measurement. The noise of the particle's movement was suppressed by smoothing the data over the frame rate, $250 \mathrm{~s}^{-1}$. An offset was applied to the bead separation based on the centre of mass calculation, shown in more detail in Section 2.6.2.1.

Figure 3.2 shows the resulting 3D-positions of two silica beads in water during an interaction measurement. The moving bead was stepped in the $y$-direction, and the position was monitored. It can be seen that when the beads are close, the stationary bead is predominantly displaced in the direction of approach ( $y$-direction). However, there is also detectable movement in the $x$ - and $z$-direction.

The trap strengths were determined by observing the variance of the Brownian fluctuations of the trapped beads. The variance of the particle position was measured when the beads are well separated for 2,000 frames. The trap strength was then 

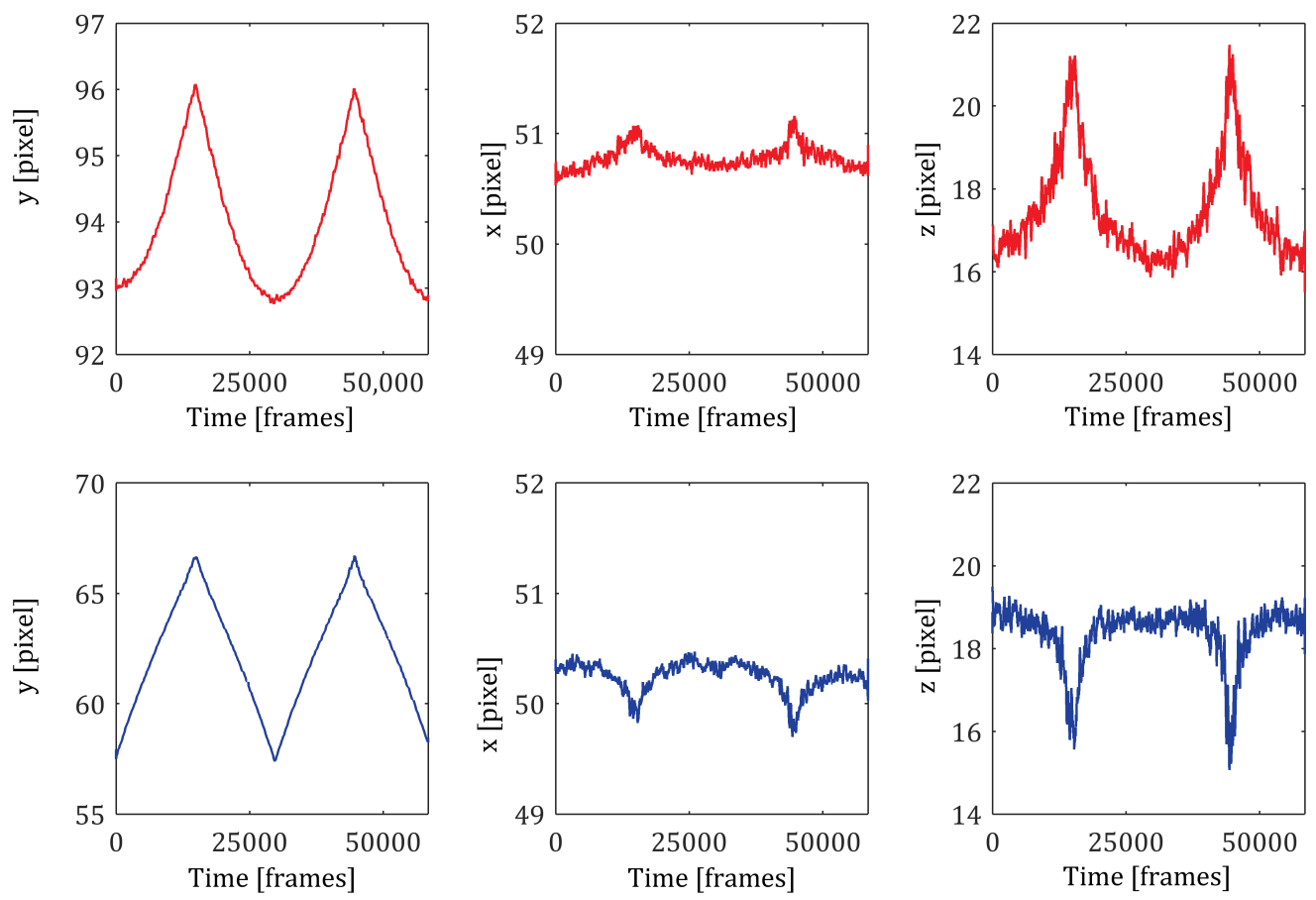

Figure 3.2.: Tracked positions of the moving bead (bottom row) and the stationary bead (top row) in the $x-, y-$ and $z$-positions. The tracked data have been corrected for drift and smoothed over the frame rate $\left(250 \mathrm{~s}^{-1}\right)$. (Note the different $\mathrm{y}$-scales)

determined by Equation 3.7, where $k$ is the trap strength, $k_{B} T$ is thermal energy and $\left\langle x^{2}>\right.$ is the variance. This method is advantageous over other methods for determining trap strength since there is no required knowledge of the hydrodynamic drag on the bead, particle size or sample viscosity, which was advantageous for measuring interactions between beads of an unknown size, described in Chapter 5 (e.g. emulsion droplets).

$$
\begin{aligned}
& k_{x}=k_{B} T\left(<x^{2}>\right)^{-1} \\
& k_{y}=k_{B} T\left(<y^{2}>\right)^{-1} \\
& k_{z}=k_{B} T\left(<z^{2}>\right)^{-1}
\end{aligned}
$$

The trap strengths in these experiments using silica beads in water were calculated using Equation 3.7 and are given in Table 3.1. The trap strengths in the moving trap 
are weaker than the trap strength in the stationary trap in the $x$ - and $y$-direction. The trap strengths match relatively well in the $z$-direction.

Table 3.1.: Trap strengths for silica beads in water when the laser power is set to $1 \mathrm{~W}$.

\begin{tabular}{l|c|c|c|c}
\hline Bead & Laser Power $(\mathrm{W})$ & $k_{x}\left(\frac{p N}{\mu m}\right)$ & $k_{y}\left(\frac{p N}{\mu m}\right)$ & $k_{z}\left(\frac{p N}{\mu m}\right)$ \\
\hline A. Stationary & 1 & 8.45 & 7.73 & 1.57 \\
B. Moving & 1 & 5.22 & 6.21 & 1.84 \\
\hline
\end{tabular}

Once the position data were tracked, and the trap strengths calculated the force between the silica beads was determined. First, the displacement between the two beads was determined using Equation 3.8, where $r$ is the separation between the two beads, $x_{B}$ is the $x$-position of the moving bead, $x_{B C}$ is the corresponding empty trap data, $x_{A}$ is the $x$-position of the stationary bead and $x_{A C}$ is the corresponding empty trap data, etc. Secondly, the force was calculated using Hooke's Law, $F=k x$, where $k$ is the trap strength and $x$ is the displacement. The force is the sum of the individual force components in the $x$-, $y$ - and $z$-directions (Equation 3.9).

$$
\begin{array}{r}
r=\left(\left|\left(x_{B}-x_{B C}\right)-\left(x_{A}-x_{A C}\right)\right|^{2}+\left|\left(y_{B}-y_{B C}\right)-\left(y_{A}-y_{A C}\right)\right|^{2}\right. \\
\left.+\left|\left(z_{B}-z_{B C}\right)-\left(z_{A}-z_{A C}\right)\right|^{2}\right)^{(1 / 2)}
\end{array}
$$

$$
\begin{gathered}
F_{r A}=k_{x A}\left(x_{A}-x_{A C}\right)\left(x_{A} / r_{A B}\right)+k_{y A}\left(y_{A}-y_{A C}\right)\left(y_{A} / r_{A B}\right)+k_{z y}\left(z_{A}-z_{A C}\right)\left(z_{A} / r_{A B}\right) \\
F_{r B}=k_{x B}\left(x_{B}-x_{B C}\right)\left(x_{B} / r_{A B}\right)+k_{y B}\left(y_{B}-y_{B C}\right)\left(y_{B} / r_{A B}\right)+k_{z y}\left(z_{B}-z_{B C}\right)\left(z_{B} / r_{A B}\right)
\end{gathered}
$$

A typical force-separation curve measured with the dual optical tweezers apparatus and discussed methodology is shown in Figure 3.3. The solid line is an exponential fit, detailed in Equation 3.5. The Debye length is found to be $109 \mathrm{~nm}$, the total charge is $3.4 \times 10^{9}$ electrons, and the resulting in the molarity of the water is 8 $\mu \mathrm{M}$ (consistent with the expectations of MilliQ water), the resolution is remarkable. 


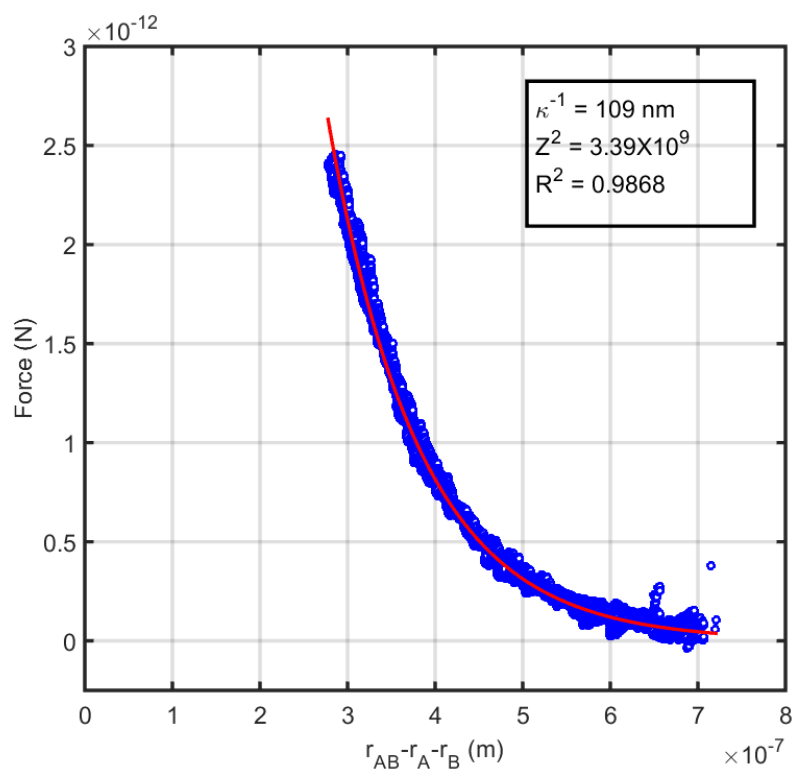

Figure 3.3.: Measured force-displacement curve of silica beads $(2 r=1.85 \mu \mathrm{m})$ in a well slide in water. The data were fitted to Equation 3.5, resulting in a Debye length of 109 $\mathrm{nm}$, charge on the droplet $(Z)$ of $3.39 \times 10^{9}$ and an ionic strength of $7.82 \times 10^{-6} \mathrm{M}$

\subsubsection{Interactions at Decreasing Debye-lengths}

To explore this method further, the interactions between silica beads was measured at decreasing Debye lengths by means of salt additions. It is well known that salt impacts the electrostatic forces between colloidal particles. An increase in salt reduces the Debye length resulting in a shorter ranged repulsion. Equation 3.2, shows the relationship between the Debye length $\left(\kappa^{-1}\right)$ and a monovalent salt at a concentration $C_{I}$, at room temperature.

The salt concentration of $\mathrm{NaCl}$ was increased from $100-5000 \mu \mathrm{M}$. The interactions were measured using the same interaction sequence and the same method detailed in Section 3.3.1. Each interaction was carried out on a different set of silica beads. We found that interactions in the same environment were reproducible and as such there appeared to be little surface heterogeneity or it was averaged over the time-scale of the measurement. 
Figure 3.4 shows the force separation curves measured in salt concentrations of 100 , $250,500,750,1000$, and $5000 \mu \mathrm{M} \mathrm{NaCl}$, again the resolution is remarkable. The corresponding exponential fits (Equation 3.5) are also shown. The $R^{2}$ values were between $0.808-0.945$, where the best fit was at $100 \mu \mathrm{M} \mathrm{NaCl}$. Most interestingly, while each interaction measurement recovered an exponential force-displacement curve indicative of the DLVO electrostatic repulsion, the corresponding decay constant bares no resemblance to the expected Debye length at higher salt concentration.
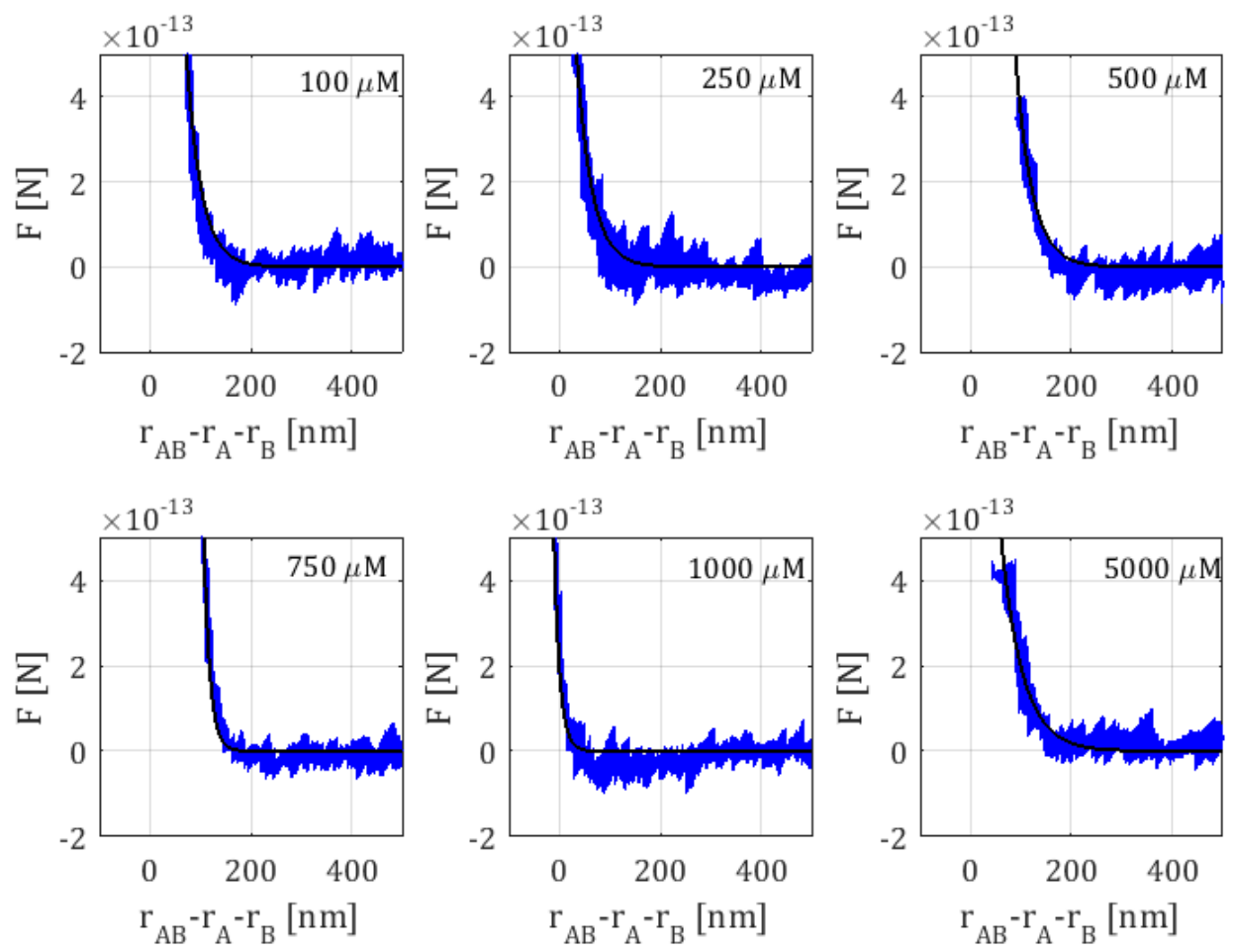

Figure 3.4.: Force-displacement curves from 100-5000 $\mu \mathrm{M}$.

To investigate this observation further, the extracted decay constant of the exponentials was plotted against the prepared salt concentration, shown in Figure 3.5. Interestingly, two regions are suggested. First, at salt concentrations from $0-100$ $\mu \mathrm{M}$, the decay constants extracted from the force curves do agree with the expected prepared salt concentrations. However, in the second region at salt concentrations above $100 \mu \mathrm{M}$ decay constants do not match the expected Debye length. Instead, 


\section{Interactions between Silica Beads}

the decay constant more closely follows the root mean square thermal fluctuations as determined by trap strength. This shows that the trap strengths available in this dual-trap optical tweezers set-up are too weak to measure interactions above $100 \mu \mathrm{M}$. If the Brownian excursions of the beads within the trap allow the beads to come into contact with one another, some particle trajectories are excluded resulting in an apparent repulsion. In this region, the length scale of the Brownian fluctuations are greater than the length scale over which the electrostatic repulsion force acts, making it impossible to measure.

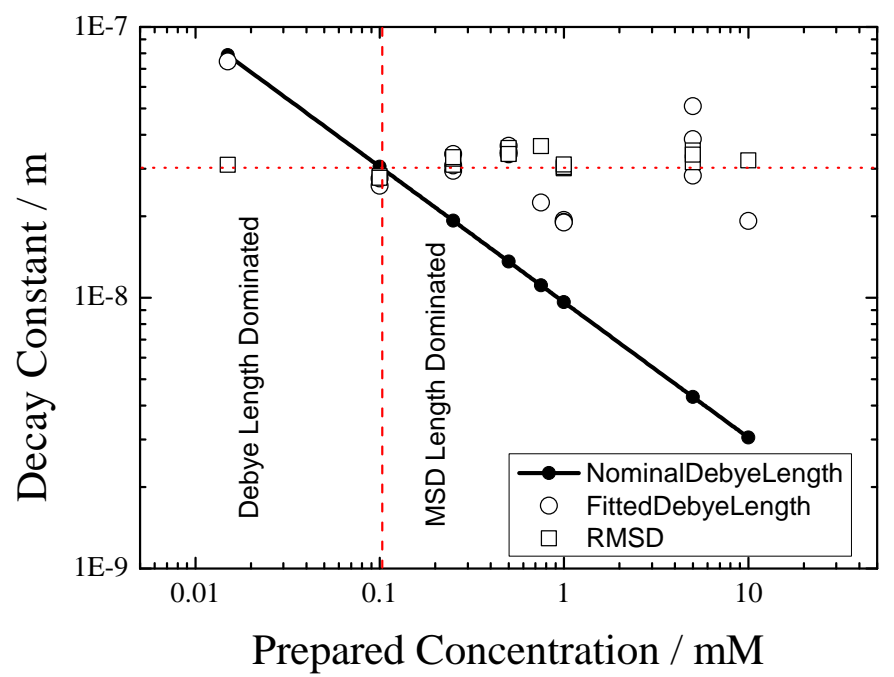

Figure 3.5.: The decay constants extracted from the force-displacement curves at its corresponding prepared salt concentration. It can be seen that the fitted Debye length $(\circ)$, aligns with the nominal Debye length $(\bullet)$ at prepared $\mathrm{NaCl}$ concentrations of 0.01 $\mathrm{mM}$ and $0.1 \mathrm{mM}$. At prepared concentrations above $0.1 \mathrm{mM}$, the decay constant follows suit with the RMSD ( $\square)$.

\subsubsection{Interactions at $>100 \mu M \mathrm{NaCl}$}

Ultimately, we would like to probe the dependence of the interactions on environmental conditions such as ionic strength, temperature and $\mathrm{pH}$, in systems such as 
emulsions where care must be taken in order to eliminate the influence of dropletto-droplet heterogeneity by repeating measurements with the exact same pair of droplets. Therefore, a method must be developed where the same pair of beads can be introduced to new environments and forces directly measured.

Once, the limitations of trap strength on achieving true force curves were understood, it was appropriate to investigate the very low salt concentrations that could practically be applied in our current apparatus. We therefore first sought to develop a way of measuring the evolution of the force curves obtained on the same set of beads during an increase of salt concentration through diffusion. This was successfully carried out using a microfluidc channel to allow salt to diffuse from an interface formed between water and $5 \mathrm{mM}$ salt solution next to which a pair of beads which were held trapped for an extended period of time.

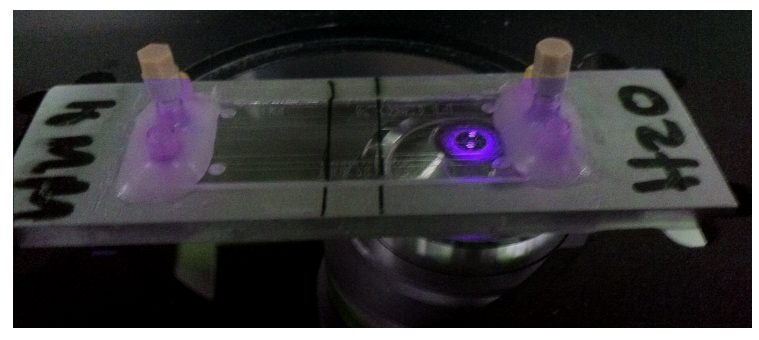

Figure 3.6.: Image of the microfludic chip used for this diffusion experiment. Water and beads were added to the right side of the chip and a $5 \mathrm{mM} \mathrm{NaCl}$ solution was added to the left side of the chip creating an interface at $1.29 \mathrm{~cm}$. (The beads were trapped where the objective is positioned in the photograph.) The chip was sealed to ensure a static environment.

Silica beads were trapped within a sealed microfluidic channel at a position away from a $5 \mu \mathrm{M} \mathrm{NaCl}$ salt/water interface and the interactions between a single pair of beads were measured over 2.8 hours. The preparation of the microfluidic chip is detailed in Section 2.4.2.4. An image of the microfluidic chip is shown in Figure 3.6, where the interaction measurement was carried out in the centre of the objective lens (1.29 $\mathrm{cm}$ from the original position of the interface). Salt diffused from left to right, and the total length of the channel is $4 \mathrm{~cm}$.

The force-displacement curves measured over time as salt diffused from the interface 


\section{Interactions between Silica Beads}

are presented in Figure 3.7. Here 13 interaction measurements were collected over 2.8 hours. Measurements were collected at times of 4, 15, 96, 101, 113, 127, 132, 138, 147, 154, 159 and 168 minutes, from the time salt was introduced into the channel creating the interface. The gap in time between 15 minutes and 96 minutes was the time where reference images were collected for 3D-tracking. As salt diffused into the water from the interface, the silica bead interactions become shorter ranged resulting in shorter Debye lengths, as expected. The force curves were fitted to the electrostatic component of the DLVO theory (Equation 3.5) and the Debye lengths, charge and corresponding salt concentration were calculated.
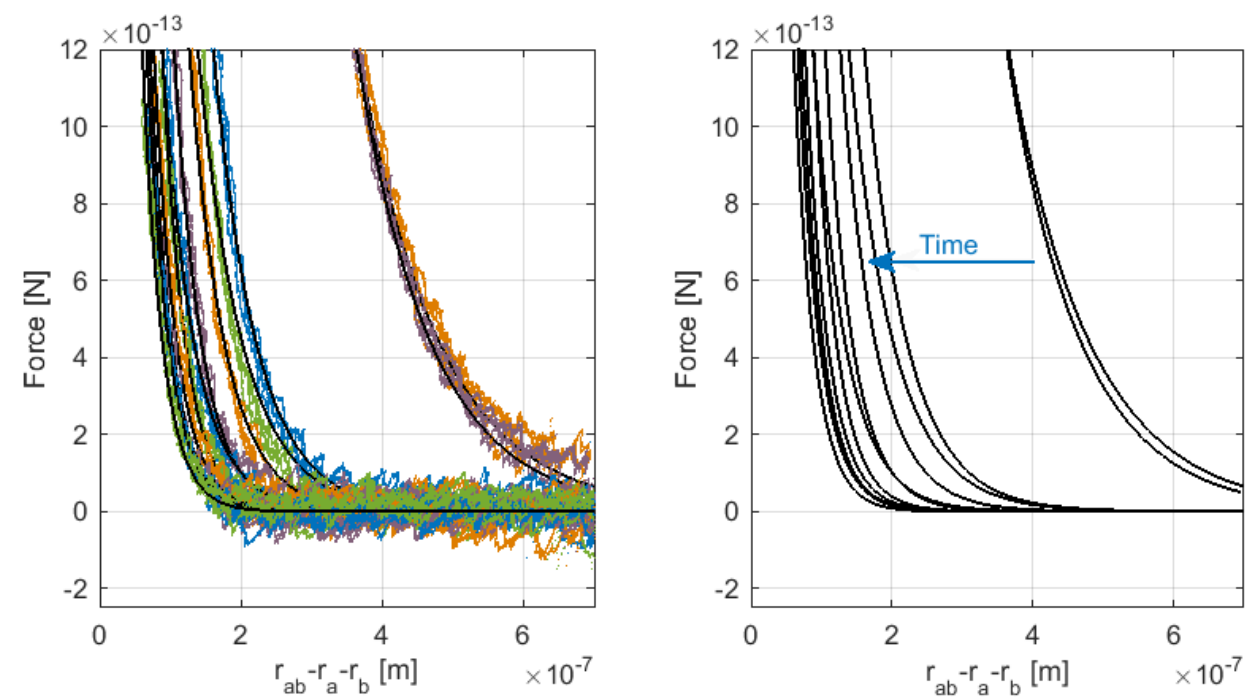

Figure 3.7.: Force displacement curves of silica beads as a function of time as the local salt concentration was increasing away from the salt-water interface. 13 measurements were taken over 2.8 hours. Left plot shows the interaction data in colour and their corresponding fits in solid black lines. For simplicity, the right plot shows only the fit data.

Table 3.2 shows the results from fitting the time-resolved measured force-displacement curves shown in Figure 3.7. The Debye length and extracted charge on the bead (Z) is presented alongside their corresponding $R^{2}$ value, which showed excellent fits to the predicted functional form for all force curves. Equation 3.2 was used to determine the salt concentration. It can be seen that the measurements were all within the 'true-force' curve range with the Debye length considerably larger than the root mean 
square excursions of the beads in the traps, so some quantitative agreement might be hoped for.

Table 3.2.: The results from fitting the force curves to Equation 3.5. The Debye length, corresponding salt concentration and charge on the bead is presented. The $R^{2}$ values show that the force curves had excellent fits.

\begin{tabular}{rrrrrrc}
\hline$\#$ & $\mathbf{T}(\mathbf{m i n})$ & \multicolumn{1}{c}{$\kappa^{-1}(\mathbf{n m})$} & $\mathbf{c}(\mu M)$ & $\mathbf{Z}\left(\times 10^{9}\right)$ & $-\zeta(\mathrm{mV})$ & $R^{2}$ \\
\hline 1 & 4 & $114.5 \pm 0.4$ & $6.9 \pm 0.1$ & $3.18 \pm 0.01$ & $121 \pm 5$ & 0.9852 \\
2 & 15 & $89.0 \pm 0.3$ & $11.4 \pm 0.1$ & $4.81 \pm 0.01$ & $104 \pm 6$ & 0.9845 \\
3 & 96 & $60.2 \pm 0.4$ & $24.8 \pm 0.3$ & $2.46 \pm 0.02$ & $58 \pm 0.5$ & 0.9699 \\
4 & 101 & $62.3 \pm 0.4$ & $23.8 \pm 0.3$ & $1.97 \pm 0.01$ & $54 \pm 0.3$ & 0.9651 \\
5 & 113 & $49.3 \pm 0.2$ & $38.1 \pm 0.2$ & $2.33 \pm 0.02$ & $48 \pm 0.2$ & 0.9792 \\
6 & 127 & $38.5 \pm 0.3$ & $62.5 \pm 0.8$ & $2.54 \pm 0.01$ & $44 \pm 0.2$ & 0.9773 \\
7 & 132 & $45.1 \pm 0.2$ & $45.4 \pm 0.6$ & $1.71 \pm 0.01$ & $47 \pm 0.6$ & 0.9746 \\
8 & 138 & $32.4 \pm 0.2$ & $86 \pm 1$ & $2.53 \pm 0.02$ & $31 \pm 0.2$ & 0.9664 \\
9 & 147 & $32.0 \pm 0.2$ & $90 \pm 1$ & $2.13 \pm 0.01$ & $36 \pm 0.2$ & 0.9827 \\
10 & 154 & $30.5 \pm 0.2$ & $97 \pm 2$ & $2.07 \pm 0.02$ & $30 \pm 0.2$ & 0.9775 \\
11 & 159 & $30.8 \pm 0.2$ & $98 \pm 1$ & $1.90 \pm 0.01$ & $27 \pm 0.2$ & 0.9763 \\
12 & 168 & $28.3 \pm 0.2$ & $115 \pm 2$ & $1.87 \pm 0.01$ & $25 \pm 0.2$ & 0.9746 \\
\hline
\end{tabular}

Figure 3.8 shows the evolution of the salt concentration extracted from the changing force curves as a function of time (inferred from the Debye lengths shown in Table 3.2). The extracted salt concentrations agreed well with the behaviour predicted by a standard one-dimensional diffusion formalism presented in Equation 3.10.

Assuming the diffusion is one-dimensional and that the initial salt concentration $\left(C_{I 0}\right)$ is maintained at the salt/water interface Equation 3.10 can be used to determine the salt concentration at a known distance from the interface $(x)$ and time $(t)$ within the microfluidic channel.

$$
C_{I}=C_{I 0} * \operatorname{erfc}\left(\frac{x}{\sqrt{4 D t}}\right)
$$




\section{Interactions between Silica Beads}

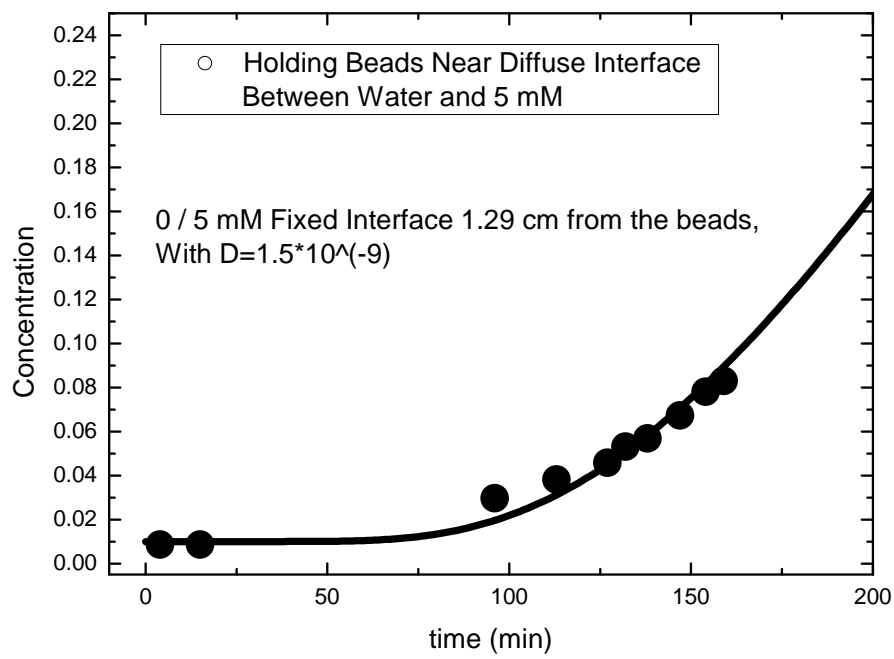

Figure 3.8.: The salt concentration extracted from the time-resolved force curves as a function of time. The diffusion formalism shows excellent agreement with the measured data.

where $\operatorname{erfc}$ is the complementary error function and $D$ is the diffusion constant $\left(D=1.5 \times 10^{-9} \mathrm{~m}^{2} \mathrm{~s}^{-1}\right)^{60}$.

\subsection{Discussion}

The interactions between silica beads have been measured at a resolution never achieved before using optical tweezers. Each interaction measurement consisted of 60,000 data points that were collected at $250 \mathrm{~Hz}$. The short exposure time of $2 \mathrm{~ms}$ allowed for near-to-real time images where the sensor was exposed at short enough bouts resulting in minimal motion blur.

The real power of this method relies both on the ability to accurately position beads at close separations as well as the capacity to determine the bead's position to sub-nanometre precision in 3-dimensions. Additionally, this method does not require 
information to be known about the viscosity of the bulk phase or information on the hydrodynamic drag of the bead. This provides the method with good flexibility with regards to the kinds of particles that could potentially be used in measurements.

Reducing the trap strength increases the resolution of the technique, allowing the applied force to yield larger averaged displacements. However, this also means the beads can travel further resulting in larger Brownian excursions. Although, these excursions are averaged, when the beads are close, some trajectories will be excluded by the presence of the other bead and this must be taken into account when interpreting the mean positions of the beads. This effect becomes evident when reducing the Debye lengths with the addition of salt. It was found that regardless of this effect, exponential force-displacement curves were recovered. However, in conditions where the Debye length becomes smaller than the RMS thermal fluctuations, the extracted decay constant bares no resemblance to the actual Debye length. Instead, the decay constant more closely resembles the RMS thermal fluctuations as determined by the trap strength. In conditions where the Debye length is sufficiently large resulting in a large electrostatic repulsion compared to the RMS Brownian excursions the force-displacement curve recovered resembles the expected Debye length. Effectively, during the interaction measurement the Brownian fluctuations of the beads should never be larger than the length scale of the Debye length, requiring therefore that the trap strength be chosen based on the expected bead trajectories.

For the optical tweezers apparatus used in this research, the maximum trap strength was already being implemented. Therefore, the boundary determined at $100 \mu \mathrm{M}$ demonstrated the largest decay constant where a genuine force curve could be recovered. However, an apparatus could be designed and built with greater ranges of trap powers that would allow for interactions at closer separations to be measured. However, with larger trap strengths there will be a reduction in resolution. Therefore, the relationship between Debye length and trap strength must carefully be considered. From the data measured here it was found that smaller trap strengths are not always better. 


\section{Interactions between Silica Beads}

The Debye lengths extracted from the fitted data from the diffusion experiment do not trend uniformly as an expected decrease in Debye length with increase in local salt concentration. The calculated uncertainties show excellent agreement with the calculated data, however, the experimental uncertainty may be larger than the presented values, which could satisfy this non-uniform trend. This experimental uncertainty could be explained by the bead-bead separation to determine the starting separation of the beads. Here, it is assumed that the beads are radially symmetric, as well the background image has been filtered of noise for the COM calculation. Also, the beads are able to freely rotate in the optical traps resulting in different point-point interactions between beads. This variation in the interaction is dependent on the beads surface heterogeneity.

\subsection{Conclusion}

In this Chapter, I have demonstrated the application of the modified dual-trap optical tweezers method to directly measure interactions between micrometre colloidal particles. Silica beads of a known size were used as a model system and the forces as a function of bead separation were directly measured; at a resolution that has never been achieved before. The interactions were first measured in a water environment where the force-distance curve agreed with the electrostatic component of the DLVO theory. Once the method was established, it was applied to salt systems with decreasing Debye lengths. Interestingly, a boundary was found where the experimental data no longer agreed with the theory. At this boundary, Brownian excursions of the particle in their traps were larger than the length scale of the electrostatic repulsion. Finally, to demonstrate the ability to measure multiple environments on the same pair of beads (at low salt concentrations where Debye lengths are faithfully recovered) the evolution of force-displacement curves was measured as the local salt concentration changed owing to the diffusion of salt from the interface, and the results obtained agreed with predictions based on a standard diffusion formalism. 


\section{Emulsion Introduction and Design}

The experiments described thus far using the modified dual-trap optical tweezers method have been focused on measuring interactions between rigid spheres. However, the question still remains, is it possible to measure interactions between two protein coated emulsion droplets using this method? Before, presenting the data on emulsion interactions I would like to first introduce emulsions, and why it is important to quantify their interactions. This chapter is split into two major sections. Firstly, a thorough introduction to emulsions is given which includes a discussion on the use of proteins as emulsifiers as well as emulsion destabilisation mechanisms. Secondly, the focus shifts to the formulation and charactersiation of the emulsion designed for direct force measurements.

\subsection{Introduction}

An emulsion is a heterogeneous system containing a mixture of two immiscible liquids, most commonly oil and water (Figure 4.1). Typically, one phase exists as discrete droplets suspended in the second, continuous phase. The energetically unfavourable interfacial layer formed between these two phases, is stabilised by a third component known as the emulsifier. The emulsifier is a surface-active molecule, 


\section{Emulsion Introduction and Design}

which reduces the interfacial tension resulting in a temporarily stabilised system. A stable emulsion does not have any noticeable changes in droplet size, aggregation or spatial arrangements over the period of observation ${ }^{61}$. All oil and water emulsions will breakdown over time, as they are not thermodynamically stable. However, they are kinetically stable over a time period that is of practical use to industry: days, weeks, months or even years. This has provided emulsions with the capability to become a major component in foods and a popular topic of study in both the fundamental and applied sciences. The majority of emulsion studies are focused on understanding the mechanisms of instability and emulsion formation. As the knowledge base has grown more studies have involved using emulsions as functional ingredients and developing novel foods.

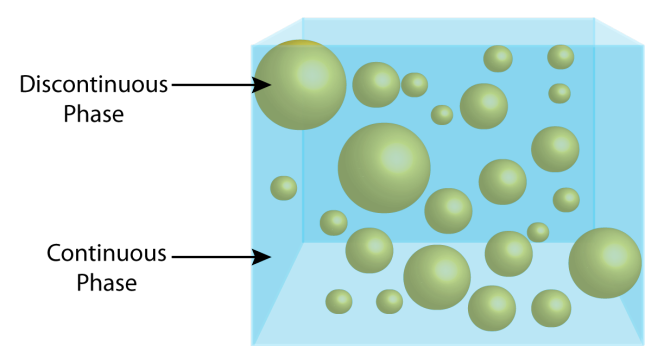

Figure 4.1.: Schematic of a oil-in-water emulsion.

\subsubsection{Emulsion Classification}

The classification of emulsions is based on the configuration of the constituent phases, the concentration of the internal phase and the particle size, which all influences the behaviour of the system and in turn defines its properties and applications.

There are three main configurations of the oil and water phases found in foods and these include oil-in-water $(\mathrm{O} / \mathrm{W})$, water-in-oil $(\mathrm{W} / \mathrm{O})$ and water-in-oil-in-water $(\mathrm{W} / \mathrm{O} / \mathrm{W})$ emulsions ${ }^{62}$. The emulsifier and concentration of each phase drive the type of emulsion that forms when energy is supplied. W/O emulsions are droplets of water suspended in oil; these emulsions make up margarine, butter and more fat-based spreads. The properties of $\mathrm{W} / \mathrm{O}$ emulsions are based principally on the 


\subsection{Introduction}

characteristics of the fat, i.e. its crystallinity and on the properties of the emulsifier. $\mathrm{O} / \mathrm{W}$ emulsions are oil droplets suspended in an aqueous continuous phase these emulsions are found in products like milk and mayonnaise. O/W emulsion properties are mainly controlled by the properties of the emulsifier and the aqueous continuous phase. Finally, much less commonly $\mathrm{W} / \mathrm{O} / \mathrm{W}$ emulsions have been used for nutrient delivery in foods. These emulsions are more complex given that they have more than one interface and that multiple osmotic pressures must be balanced.

Additionally, emulsions are classified by the concentration of the dispersed phase. For $\mathrm{O} / \mathrm{W}$ emulsions, this is known as the oil fraction $(\phi)$. Emulsions with a low oil fraction of less than $<0.58$ are classified as dilute emulsions, i.e. milk. The physiochemical properties of emulsions drastically change as their oil fraction is increased, as shown in Figure 4.2. In fact, due to the interactions between droplets the emulsions can change from a liquid to a more solid gel-like appearance and texture. Emulsions with a more solid or gel-like appearance and texture are classified as concentrated emulsions. When the oil fraction is at $>0.64$ droplet interactions become increasingly important, and the properties of the internal phase become significant, i.e. cream or mayonnaise.

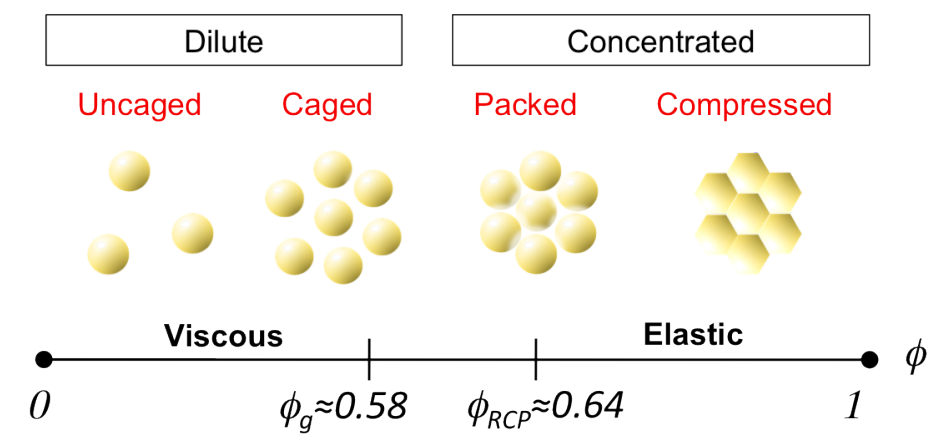

Figure 4.2.: Schematic of the effect of concentration of internal phase on the viscoelastic properties from dilute to concentrated emulsions. $\phi_{g}$ is the oil fraction where there is a glass transition state where the droplets become caged by their neighbours and $\phi_{R C P}$ is the oil fraction where droplets become randomly closed packed where the interfaces become compressed and deformed ${ }^{63}$.

Finally, emulsions can be classified based on particle size. The particle size distribution of an emulsion affects the rheological properties, the visual appearance and 


\section{Emulsion Introduction and Design}

the stability of the system. Emulsions are either classed as macro or microemulsions. A few of the similarities and differences between these two different classes of emulsions are outlined in Table 4.1. The most significant difference between the properties of micro and macroemulsions concern the thermodynamic and storage stability. Microemulsions are considered thermodynamically stable due to their small droplet size. Generally speaking, their storage stability is considered to be infinite, even though this cannot be experimentally verified. However, macroemulsions will separate. Hereon, the definition of an emulsion will be defined as a macroemulsion.

Table 4.1.: A comparison of Macro and Microemulsions ${ }^{64}$

\begin{tabular}{lcc}
\hline Property & Macroemulsion & Microemulsion \\
\hline Components & Oil-water-emulsifier & Oil-water-emulsifier \\
\hline Number of Emulsifiers & One or more & One or more (usually 2+) \\
\hline Surfactant Types & All & All \\
\hline Surfactant Concentration & Fairly low & Fairly high \\
\hline Droplet Size & Micrometres & $0.01-0.001 \mu \mathrm{m}$ \\
\hline Thermodynamically stable & No & Yes \\
\hline Storage Stability & Formulation-dependent & Infinite* \\
\hline \multicolumn{2}{c}{ cannot be experimentally verified } \\
\end{tabular}

\subsection{Emulsion Formation}

Emulsion formation is a non-spontaneous process; energy is required in order to make a kinetically stable system. One of the ways to provide this energy is by a mechanical process that disperses one phase within another by increasing the surface area by a large amount. The amount of additional free energy $\left(\triangle G_{f}\right)$ required to form an emulsion with an increased interfacial area $(\triangle A)$ is given in Equation $4.1^{65,66}$.

$$
\triangle G_{f}=\gamma \triangle A-T \Delta S_{f}
$$


where $\gamma$ is the interfacial tension, $T$ is temperature and $\Delta S_{f}$ is the entropy of formation. Emulsion instability is a result of the energy penalty associated with the large interfacial area of the droplets within the emulsion which is given by $\gamma \triangle A$. This energy term outweighs the entropy term associated with the formation of the droplets from the bulk phases. $\Delta S_{f}$ is relatively small in entropic terms due to the number of droplets formed. This results in $\Delta G_{f} \geq 0$ resulting in a thermodynamically unstable system $^{66}$.

Homogenisation is the mechanical process used to convert bulk oil and water phases into a kinetically stable emulsion ${ }^{67}$. There are some excellent reviews, which discuss emulsion formation ${ }^{68}$ and the equipment used to make emulsions ${ }^{69}$. In industry, homogenisation is achieved by applying intense mechanical agitation to a heterogeneous mixture using a mechanical device known as a homogeniser ${ }^{69}$.

Table 4.2 shows a comparison of the homogenisers utilised in the food industry. These homogenisers differ in the type and amount of energy supplied, the rate of processing and the droplet size produced. Although, ultrasonic and membrane systems produce a more monodisperse system, they are not an economical option for industrial use due to low product throughput. Most research on emulsion formation has been focused on reducing droplet size, polydispersity and the energy required for emulsion formation $^{68-70}$.

Table 4.2.: Comparison of common homogenisers in the food industry

\begin{tabular}{lcc}
\hline Type of Homogeniser & Mean droplet diameter (um) & Production \\
\hline Rotor-stator-system [shear mixer] & $>2$ & Industrial scale \\
\hline Rotor-stator-system [colloidal mill] & $<1$ & Industrial scale \\
\hline High pressure homogeniser & $>0.2$ & Industrial scale \\
\hline Ultrasonic-systems & $\sim 0.4$ & Laboratory scale \\
\hline Membrane-systems & $\sim 0.2$ & Laboratory scale \\
\hline Microfluidics & $>10$ (monodispersed) & Laboratory scale \\
\hline
\end{tabular}




\section{Emulsion Introduction and Design}

During emulsion formation, the various emulsifiers become rapidly absorbed at the interface of the newly formed oil droplets. This results in an immediate steric stabilising layer, which protects the droplets against re-coalescence and subsequently provides physical stability to the emulsion during later processing and long-term storage.

\subsection{Interfacial Layer}

Emulsifiers are surface-active molecules. These are most commonly amphiphilic proteins, polysaccharides, phospholipids and small molecule surfactants. In formulating an emulsion the choice of emulsifier(s) is key as emulsifiers vary greatly in their effectiveness of producing small droplets during homogenisation (i.e. surface activity) and also their ability to resist change right through from harsh processing to consumption (i.e. stability). Additional challenges arise when developing foods as cost, availability, ease of use, compatibility with other ingredients and "label friendliness" must also be considered ${ }^{71}$. Therefore, there is no single emulsifier that is ideal for all systems ${ }^{72,73}$. Consequently, considerable research has been undertaken on emulsifiers to better understand their structure and their ability to bind to an interface to obtain a stable system.

The predominant thermodynamic driving force for the adsorption of proteins on the interface is the removal of hydrophobic residues from the unfavourable aqueous environment. This leads to the rearrangement or even unfolding of the proteins at the interface, as they minimise unfavourable interactions ${ }^{7 / 4}$. The extent of the unfolding and rearrangement to form a stabilising layer is dependent on the flexibility of the protein molecule, which is determined largely by the strength of the forces maintaining the secondary and tertiary structures. The effectiveness of the protein to bind to the interface and reduce the interfacial tension is determined by how many hydrophobic residues it has and also the type of residues and their availability to the interface. 
Milk proteins are often used as emulsifiers in the food industry due to their abundance, "label friendliness", high nutritional quality and their natural amphiphilicity. Within milk there are two classes of proteins: caseins (i.e. $\beta, \alpha$, and $\kappa$ ) and whey proteins (i.e. $\beta$-lg or $\alpha$-lactalbumin). The surface activities of milk proteins from most surface active to least surface active are: $\beta$-casein $>$ monodisperse casein micelles $>$ serum albumin $>\alpha$-lactoglobulin $>\alpha$-caseins $=\kappa$-caseins $>\beta$ - $\lg ^{75}$. In this Ph.D. programme two different proteins were investigated, $\beta$-lg and a sodium caseinate protein mixture, as potential candidates to prepare an emulsion with specific properties suitable for optical tweezers experiments. The following sections provide a discussion on the structure and function of $\beta$-lg and sodium caseinate when used as emulsifiers to produce $\mathrm{O} / \mathrm{W}$ emulsions.

\subsection{1. $\beta$-Lactoglobulin}

$\beta$-lg is the least surface active out of all the milk proteins due to its strong intermolecular forces that mean it maintains its tertiary and secondary structure during emulsification and takes a long time to configure onto an $\mathrm{O} / \mathrm{W}$ interface. Due to these strong intermolecular forces $\beta$-lg can be regarded as a pseudo two-dimensional system of densely packed deformable particles at an interface ${ }^{76}$. Rearrangement of $\beta$-lg on oil-water interfaces takes 4-5 hours during which the protein relaxes on the interface and spreads about $25 \%$. The amount of unfolding is dictated by the congestion of protein molecules on the interface where molecules that initially bind to the interface during homogenisation do not have time to unfold before additional molecules adsorb ${ }^{76}$. Kim et al. found that the higher concentration of protein the greater the adsorption rate and the less unfolding whereas at a low protein concentration the adsorption rate was low and the unfolding rate high. This results in different surface tension: at high protein concentrations where there is less unfolding due to the densely packed globular proteins the surface tension was higher, whereas at low concentrations there is a less dense layer ${ }^{77}$. Regardless, the interfacial layer formed by $\beta-\lg$ is a thin and dense layer of protein of a thickness of $2-3 \mathrm{~nm}$ at neutral 


\section{Emulsion Introduction and Design}

$\mathrm{pH}^{78,79}$.

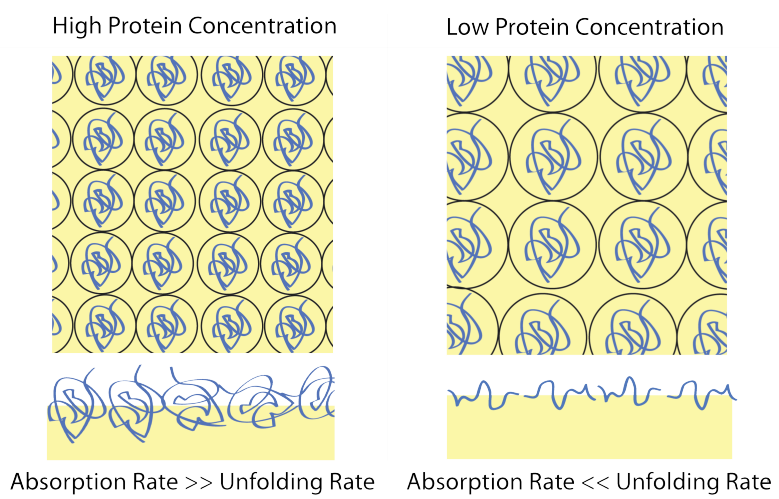

Figure 4.3.: The influence of protein concentration during homogenisation on the unfolding of absorbed proteins. At low protein concentration, the absorbed $\beta$-lg molecules have more time to unfold before the additional proteins absorb which leads to greater conformational changes. Figure reproduced with permission from Kim et al. ${ }^{77}$

Zhai et al. studied the conformation of $\beta$-lg using a combination of dual polarization interferometry (DPI), synchrotron radiation circular dichroism spectroscopy (SRCD) and front-face fluorescence spectroscopy to measure in situ the dimensions and the conformation of $\beta$-lg adsorbed at the hexadecane-water and tricaprylin-water interfaces $^{80}$. Using DPI they deposited $\beta$-lg onto silicon oxynitride and also a C18 surface. As expected they observed a moderately dense $\left(0.37 \mathrm{~g} / \mathrm{cm}^{3}\right)$ layer of $\beta-\mathrm{lg}$ with a thickness of $3.6 \mathrm{~nm}$ on silicon oxynitride whereas on the hydrophobic C18 a denser $\left(0.98 \mathrm{~g} / \mathrm{cm}^{3}\right)$ thinner layer $(1.1 \mathrm{~nm})$ of $\beta-\lg$ resulted ${ }^{80}$. This result suggests that the $\beta$-lg remained intact on the silicon oxynitride surface as the thickness agrees with the crystal structure reported in the literature ${ }^{80}$. However, there was significant unfolding on the hydrophobic C18 surface where $\beta$-lg had become a more open and flat structure resulting in a denser monolayer ${ }^{80}$. In agreement with the DPI data, the SRCD and front-face fluorescence spectroscopy confirmed that the adsorption of $\beta$-lg onto an oil-water interface induces unfolding with a loss of tertiary structure $^{80}$. The conformational changes observed included a loss of the $\beta$-sheet structure and an increase in $\alpha$-helical structure ${ }^{80}$. This structural rearrangement allows for the non-polar groups to orient toward the oil phase, which enhances 
hydrophobic interactions ${ }^{80}$.

$\beta$-lg has three genetic variants $A, B$ and $C$. Euston et al. have studied the emulsifying properties of these three variants ${ }^{81}$. They discovered the emulsifying ability of $A$ is the greatest and $C$ is the least. The most common variants are $A$ and $B$ where $C$ has only been found in Jersey cows ${ }^{81}$. These variants differ in their amino acid sequence at positions ${ }^{7 / 4}, 64$ and 118 along the amino acid backbone shown in Table 4.3. ${ }^{81-83}$. These substitutions in the amino acid backbone lead to the difference in net charge on the molecules ${ }^{81}$. Studied at $\mathrm{pH} 7, \beta-\lg A$ has a more negative charge due to the aspartic acid (pKa 3.86) and is most susceptible to proteolytic hydrolysis, whereas $\beta$ - $\lg C$ has the lowest net negative charge and is less susceptible to tryptic hydrolysis ${ }^{81}$. Although these differences were observed they showed the greatest differences at low concentrations. ${ }^{81}$ Typically in food processing the $\beta$-lg supplied is in the form of Whey protein where the $\beta$-lg only is about $50 \%$ of the protein mixture and in industry much higher concentrations are used than in the Euston et al. study. Therefore, the effect of having different variants in the protein mixture is reduced ${ }^{81}$.

Table 4.3.: Summary of the amino acid variations of the three genetic variations of the $\beta$-lg.

\begin{tabular}{clll}
\hline Blg variant & \multicolumn{3}{c}{ Amino Acid Position } \\
\hline & 59 & 64 & 118 \\
\hline$A$ & Glutamine & Aspartic Acid & Valine \\
\hline$B$ & Glutamine & Glycine & Alanine \\
\hline$C$ & Histidine & Glycine & Alanine \\
\hline
\end{tabular}

The conformation of the protein on the interface is dependent on the available functional groups and the environment surrounding the protein. The ionic strength, acidity, ions, polarity, and temperature are a few important properties that can affect protein conformation. An environment that is more or less acidic then the isoelectric point of the protein affects the charge distribution, as amino acids can become either protonated or deprotonated depending on their pKa. The amino acids 


\section{Emulsion Introduction and Design}

that contribute to the charge density in $\beta-\lg$, are arginine, aspartic acid, glutamic acid, histidine and lysine. These amino acids make up $53 \%$ of the protein structure and therefore, dominate in defining the protein conformation. In adsorbed protein layers, carboxylic acid groups tend to reside closer to the hydrophobic particle surface than do amine groups. The sign and magnitude of the average surface charge density is dependent on the $\mathrm{pH}$. The isoelectric point of $\beta$-lg is about $\mathrm{pH} 5.2^{84}$. The surface charge, when below the pKa, will be positive due to protonation whereas above the pKa $\beta$-lg will be negatively charged.

\subsubsection{Caseins}

Caseins are a family of non-globular milk proteins consisting of four genetic variants including $\alpha_{s 1}, \alpha_{s 2}, \beta$ and $\kappa$-casein. Often caseins are described as flexible complex linear copolymers because their solution behaviour is similar to synthetic block copolymers. They have the ability to self-assemble into various aggregated states depending on the solution properties. The self-assembly of caseins into casein micelles is still highly debated between scientists as a single model has yet to be agreed upon. However, beyond the self-assembly of caseins in solution these proteins have an ability to stabilise interfaces due to their structure and amphiphilic properties.

Caseins yield an entangled monolayer of flexible chains at a hydrophilic-hydrophobic interface. This disordered entanglement at the interface is due to the high proportion of hydrophobic residues like proline and the absence of cysteine residues that typically disrupt secondary structure yielding flexible linear proteins ${ }^{85}$. This combination of disorganized structure and substantial hydrophobicity coupled with the relatively low molecular weight enables caseins to spread rapidly at the oil-water interface. Caseins are also phosphoproteins, and each protein variant is phosphorylated to a varying degree. Interestingly, the phosphoseryl residues and the hydrophobic residues are not distributed evenly along the peptide backbone ${ }^{85}$. This definitive arrangement yields a distinct amphiphilic nature to the casein proteins where there are distinct pockets of hydrophobic and hydrophilic behaviour making caseins an excellent emulsifier. 
Individually, the genetic variants of casein behave differently at an interface mainly due to the organization of phosphate residues along the amino acid backbone. In Figure 4.4 the amino acid sequence of each casein variant is depicted where the phosphate residues are labelled with a $P$ after the amino acid indicating a phosphorylation site. Serine $(S e r)$, an amino acid containing a hydroxyl group is highly susceptible to phosphorylation resulting in a more polar amino acid. This results in distinct hydrophilic segments along the amino acid backbone. It is evident that the clusters and arrangement of phosphate residues vary based on each variant. Whereas the $\kappa$ has a single phosphate residue $\beta, \alpha_{s 1}$ and $\alpha_{s 2}$ have several clusters of 2,3 or 4 phosphate groups which results in an protein orientation where these hydrophilic segments protrude into the solvent phase either as loops or tails. $\beta$ casein contains 5 phosphoserines which are located at the N-terminus yielding a tail of approximately 40-50 amino acid residues extending into the aqueous phase whereas the remaining residues are mainly hydrophobic resulting in a 'train' across the interface (Figure $4.4 \mathrm{~A}$ ). In comparison $\alpha_{s 1^{-}}$and $\alpha_{s 2^{-}}$casein contains clusters of phosphoserines mid-sequence resulting in loops protruding into the aqueous phase (Figure $4.4 \mathrm{~B}$ ). This amphiphilic structure is the molecular basis of caseins having excellent emulsifying properties.

Studies conducted on the genetic variants of caseins and sodium caseinate have shown that caseins have the ability to cover an interface to the extent of 2-3 mg $\mathrm{m}^{-2}$, similar to whey proteins. When using sodium caseinates as an emulsifier there seems to be no preferential binding to the interface between caseins however, when using mixtures of purified caseins a preferential binding was observed between $\alpha_{s 1}$ and $\beta$-caseins where the former was much less.

Beyond the unique amphiphilic properties of caseins, their electrostatic properties are also important in creating a stable emulsion. The average isoelectric point $(\mathrm{pI})$ of caseins is approximately $\mathrm{pH} 4.5$.

In this study, casein was used in the form of sodium caseinate that is comprised of a soluble mixture of disordered hydrophobic caseins. Made by precipitating out the 


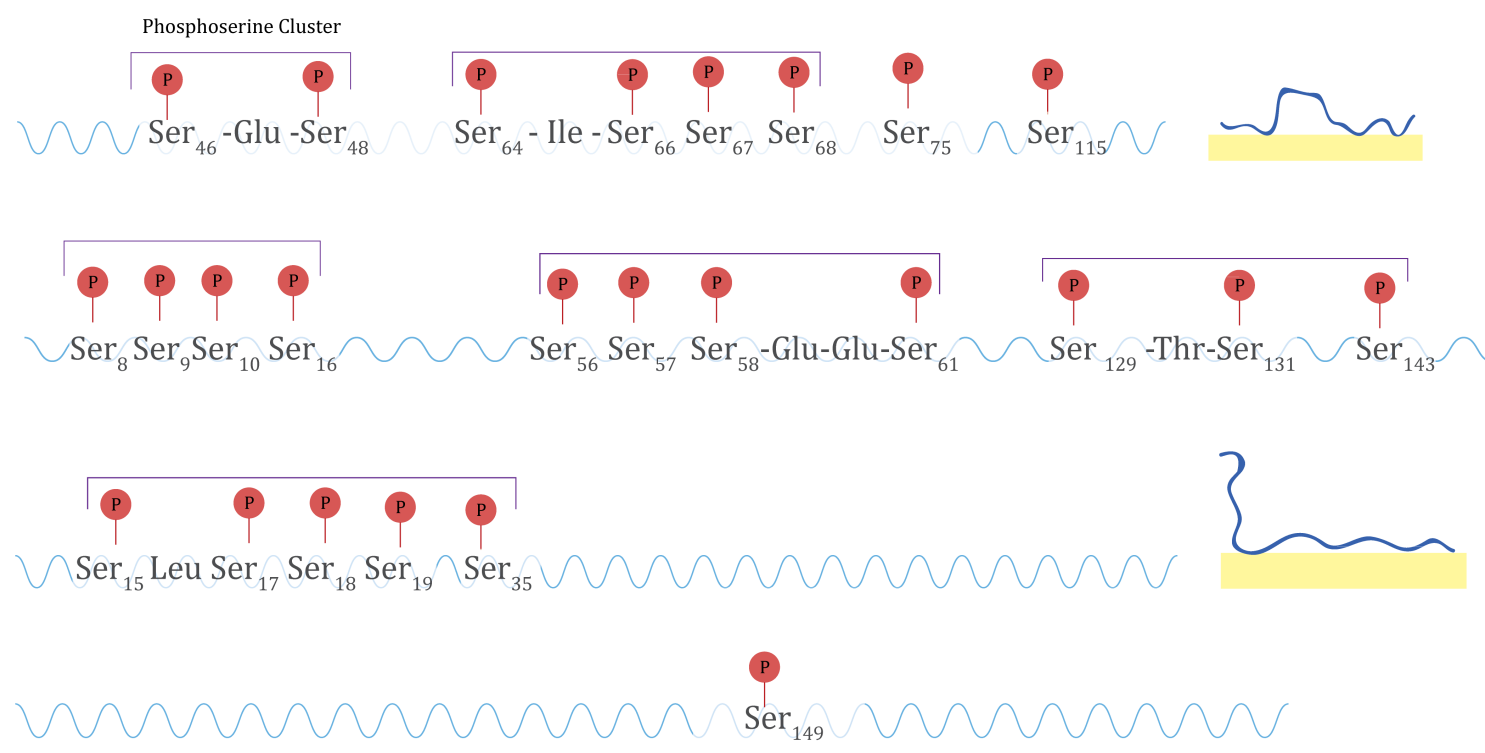

Figure 4.4.: Depiction of the 'train-loop-tail' model of $\beta$-casein (A), $\alpha_{s 1, s 2}$-casein (B) and $\beta-\lg (\mathrm{C})$.

proteins from skim milk by reducing the $\mathrm{pH}$ of the milk to 4.6 (yielding aggregated proteins known as the curds) ${ }^{86}$. The curd is then heated to $50-55{ }^{\circ} \mathrm{C}$ then washed and dewatered to a moisture content of 50 to $60 \%$. The dewatered curd is then dried further using horizontal vibrating fluid bed driers, and this results in a product known as acid casein ${ }^{86}$. Finally, sodium caseinate is produced by solubilising the acid casein with $\mathrm{NaOH}$ by increasing the $\mathrm{pH}$ to $6.7-7.0$ and spray-drying this solution. ${ }^{86}$.

\subsection{Mechanisms of Instability}

If the attractive forces dominate the system the emulsion droplets will collide resulting in aggregation or the rupturing of the interfacial film yielding fewer and larger droplets. In producing products for consumers, a stable product is necessary and, therefore, an understanding of the mechanisms, that result in emulsion destabilization is fundamental to be able to control such processes. Below is an explanation of the five 
4.4. Mechanisms of Instability

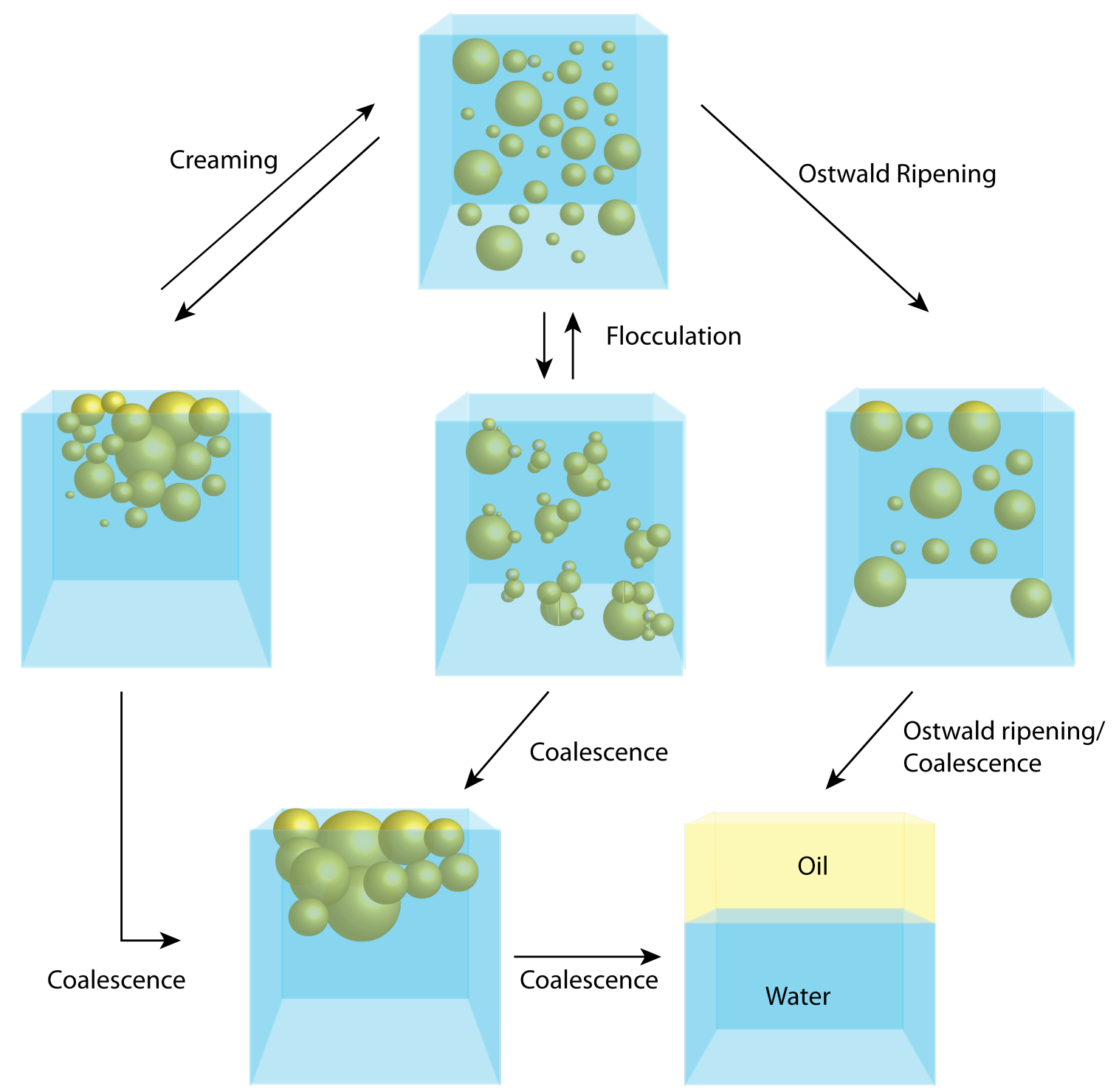

Figure 4.5.: Illustration of the coalescence process in oil-in-water emulsions ${ }^{61}$ 


\section{Emulsion Introduction and Design}

mechanisms of instability in emulsions including; creaming, coalescence, flocculation, Ostwald ripening and phase inversion.

\subsubsection{Coalescence}

Coalescence is an irreversible event where droplets come together and form larger droplets. This is initiated by the collision of droplets and often occurs in the cream layer or while droplets are aggregated. The properties of the interfacial layer dictate if the droplets will aggregate, coalesce or rebound once the collision occurs (Figure 4.6). This is dependent of the attractive van der Waals forces and also the repulsive forces present. If attractive forces dominate the film will be unstable, the liquid will drain from the film, and the film will thin ${ }^{61}$. If the film thins to its critical thickness $\left(h_{\text {crit }}\right)$ it will rupture, and the droplets will join resulting in coalescence ${ }^{61}$. If repulsive forces dominate, the droplets will repel each other.

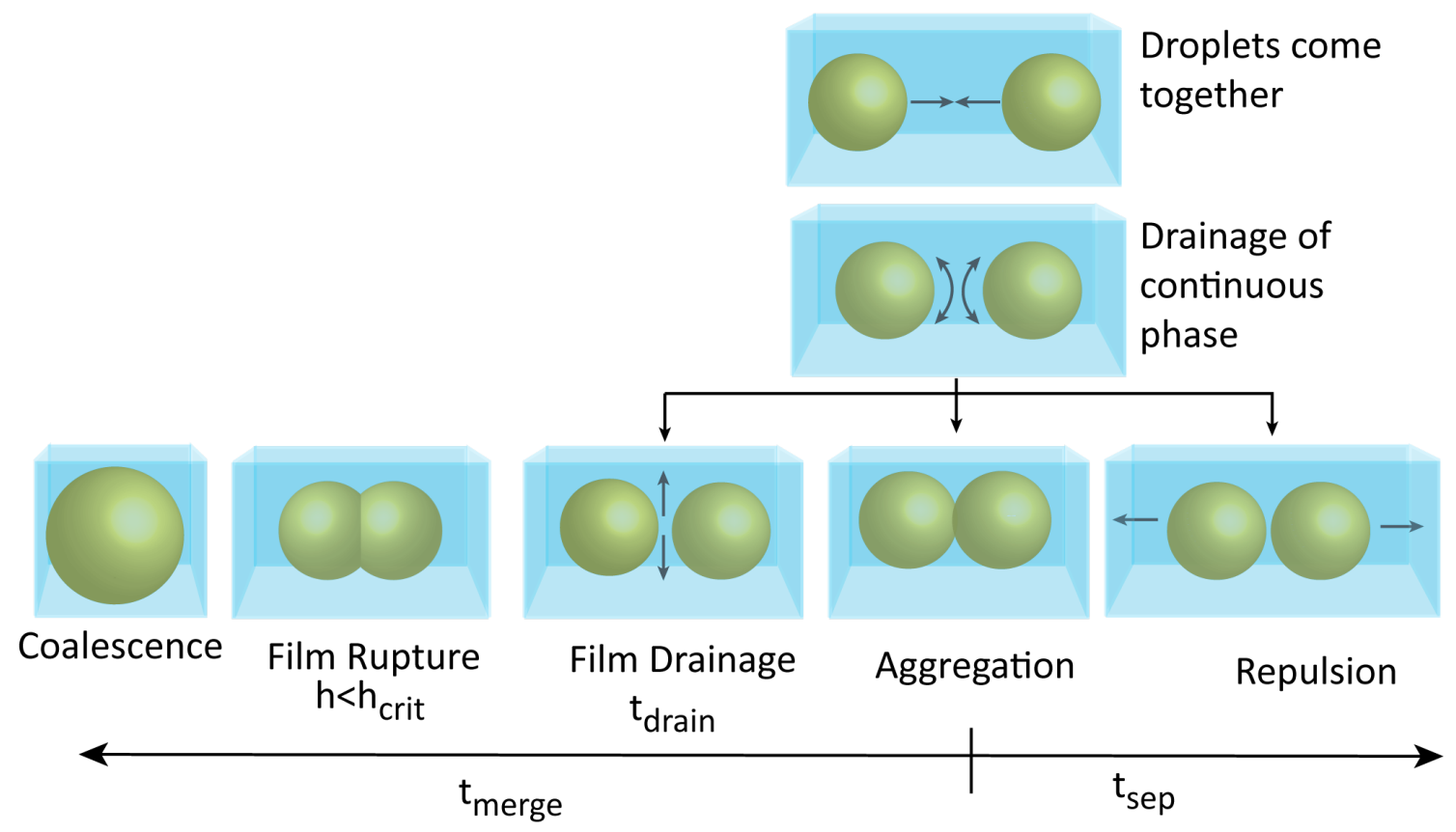

Figure 4.6.: Illustration of the coalescence process in oil-in-water emulsions ${ }^{61}$

Coalescence occurs after droplets have been close together for an extended amount 
of time for example within a floc or a cream layer.

\subsubsection{Creaming}

Creaming is the phenomenon whereby oil droplets rise due to the density difference between the oil and water phases without a change in droplet size (Figure 4.7). Creaming is reversible, and the original uniform distribution of droplets can be obtained by gentle mixing.

$$
v_{\text {stokes }}=\frac{2 r^{2}\left(\rho_{1}-\rho_{2}\right)}{9 \eta}
$$

Stoke's Law explains this phenomenon (Equation 4.2). Here $v_{\text {stokes }}$ is the velocity of creaming, $r$ is the radius of the oil droplet, $\rho_{1}$ and $\rho_{2}$ are the density of the continuous phase and dispersed phases, respectively and $\eta$ is the shear viscosity of the continuous phase. Decreasing the size of the droplets, decreasing the difference in density between the phases and increasing the viscosity of the continuous phase can reduce the rate of creaming. Often creaming is a result of other destabilization mechanisms including flocculation and coalescence where the size of the droplets or aggregations become large resulting in an increase in the rate of creaming. Although, creaming is often undesirable, in some food processes this instability is required (i.e. the separation of cream in milk).

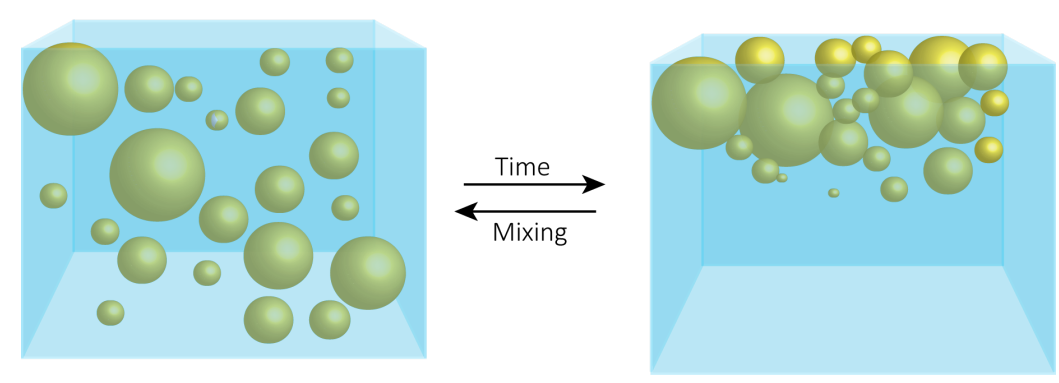

Figure 4.7.: Creaming of an oil-in-water emulsion. 


\section{Emulsion Introduction and Design}

\subsubsection{Flocculation}

Flocculation is a reversible event where two or more droplets weakly aggregate due to the influence of a local minimum formed by the competition between attractive van der Waal forces and long-range repulsive interactions ${ }^{87}$. It is often induced by the concentration of emulsifier not being at the critical concentration whereby each oil droplet is properly coated. If the critical concentration is not met and the emulsifier is polymeric then bridging flocculation can occur as the biopolymer present becomes shared between oil droplets and bridges between neighbouring droplets as depicted in (Figure 4.8). Bridging flocculation can also occur when there may be sufficient emulsifying biopolymer, however; for example if the biopolymer is unable to attach to the surface of the droplet in time before the collision with neighbouring droplets occurs. At the other extreme where there is too much biopolymer in solution depletion flocculation can take place. Unabsorbed biopolymer in solution is unable to fit between emulsion droplets creating an osmotic force between the bulk continuous phase and the interdroplet space resulting in flocs.
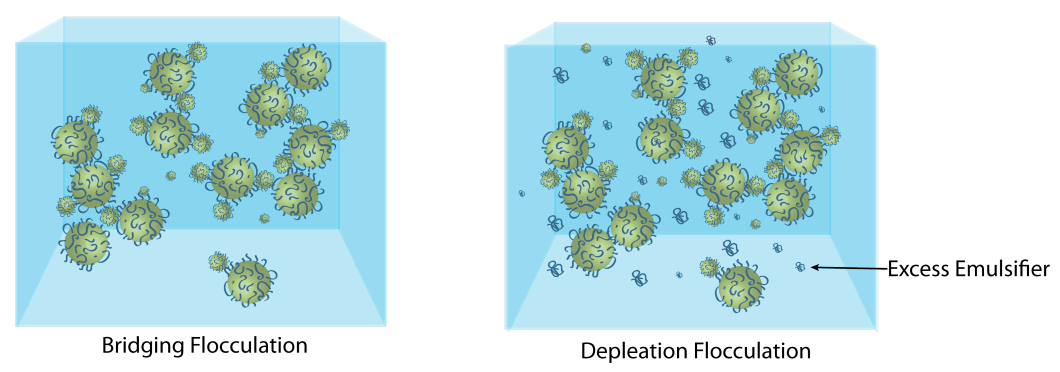

Figure 4.8.: Illustration of bridging and depletion flocculation.

\subsubsection{Ostwald Ripening}

Ostwald ripening describes the coarsening of an emulsion by molecular diffusion. It is the process where large droplets grow at the expense of smaller ones because of mass transport of dispersed phase from one droplet to another through the intervening continuous phase (Figure 4.9) ${ }^{87}$. For an emulsion to undergo Ostwald ripening, 
a few considerations must be satisfied. This includes that the molecules of the discontinuous phase must have a significant diffusion coefficient in the continuous phase, allowing for the transfer of a molecule from one droplet through the continuous phase to a second larger droplet. If the diffusion coefficient is sufficiently low, then the diffusion will be slow and not measurable. Beyond, the transfer of the molecule from the droplet to the continuous phase the molecule must be able to bypass the interface. Therefore, for Ostwald ripening to occur the molecule must be able to diffuse through the interfacial layer.

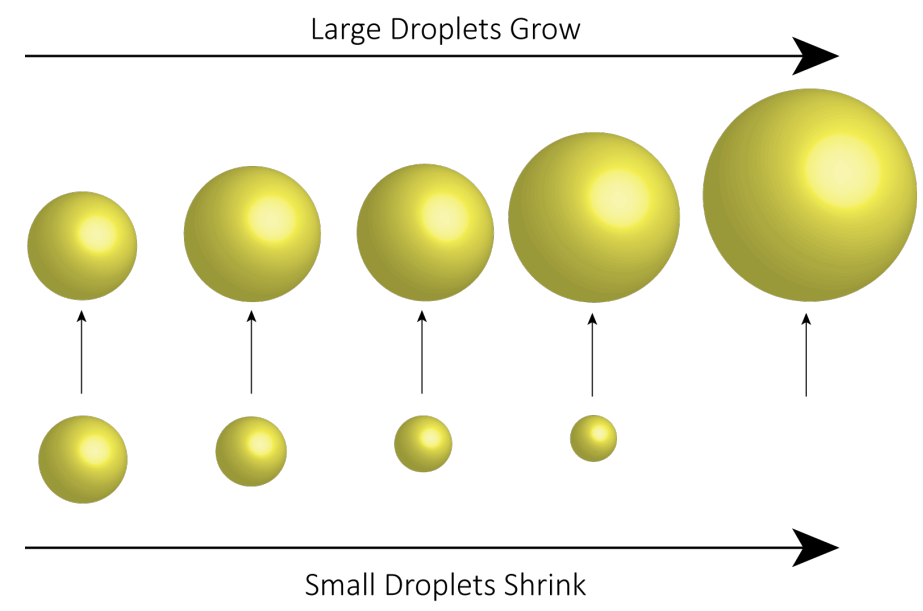

Figure 4.9.: Illustration of the Ostwald ripening process. The large oil droplets grow at the expense of the smaller droplets ${ }^{87}$.

Ostwald ripening can be controlled by narrowing the droplet size distribution resulting in a similar pressure gradient at all interfaces (Laplace pressure), increasing the viscosity of the continuous phase and adjusting the formulation of the emulsion by incorporating a third component which is soluble in the dispersed phase but not in the continuous phase.

It is difficult to differentiate between coalescence and Ostwald ripening because both processes result in irreversible droplet growth. To experimentally distinguish between the two instability processes droplet size can be tracked over time using light scattering and particle size measurements. A typical pattern of droplet growth for coalescence is that the reciprocal of the droplet diameter squared should decrease 


\section{Emulsion Introduction and Design}

linearly with time $\left(1 / \mathrm{d}^{2} \propto \mathrm{t}\right)$ whereas for Ostwald ripening the droplet diameter cubed usually increases with time $\left(\mathrm{d}^{3} \propto \mathrm{t}\right)^{88}$.

The emulsions, which are being investigated in this study, are not prone to this destabilisation. This is because the solubility of the lipids in water is so low that the mass transport rate is negligible ${ }^{87}$. Emulsions, where Ostwald ripening may be observed, are those emulsions containing more water-soluble lipids (i.e. flavour oils) or if the aqueous phase contains alcohols ${ }^{87}$. 


\subsection{Formulation \& Characterisation}

\subsection{Formulation \& Characterisation}

The emulsions produced for the direct force measurements carried out in this thesis must meet strict criteria to maximise the changes of success. These criteria have been determined through the experience of trapping and manipulating droplets with the optical tweezers apparatus and mainly involves droplet size, polydispersity and refractive index.

\subsubsection{Emulsion Criteria}

For our efforts to develop a method to measure interactions between micrometre sized droplets in order to understand the complex interactions found in food structures a conventional emulsion system was chosen; a protein stabilised $\mathrm{O} / \mathrm{W}$ emulsion. Two proteins $\beta$-lg and sodium caseinate were trailed based on their plenitude, popularity and most importantly they are well characterised in literature and known to be superior emulsifiers (See Section 4.3). The oil was also kept food grade with soybean oil, chosen for its abundance as well as it matching the refractive index requirements for optical trapping that are discussed further in Section 2.2.1. The free parameters in the emulsion design were the concentration of the oil, emulsifier and continuous phase along with the ionic strength and $\mathrm{pH}$.

Beyond the ingredient restrictions of emulsifier and oil, the most important criteria the emulsion had to meet for our study was the particle size. The emulsion droplets needed to have a diameter from $1 \mu \mathrm{m}$ to $5 \mu \mathrm{m}$. Droplets with a diameter less than $1 \mu \mathrm{m}$ are more difficult to trap in the optical tweezers and they also possess larger Brownian fluctuations making it difficult to measure the force. Large droplets over 5 $\mu \mathrm{m}$ are quick to cream to the slide surface out of the focal range of the trap.

Controlling the particle size distribution is also important because our starting point was that measurements should be carried out on pairs of droplets of similar size. For the OT studies, the emulsions are diluted enormously to only a few droplets per 100 


\section{Emulsion Introduction and Design}

$\mu \mathrm{L}$ and if there is a broad distribution statistically there will less of a chance to find two droplets of similar size. Populations of droplets in the sub-micrometre region also create a multitude of problems in force measurements i.e. interfering with the trapped droplets of interest either by sticking to the surface or interfering within the trap. As an observation throughout this work, particularly in initial trials, smaller droplets would often decorate the surface of larger droplets.

Finally, the emulsion must be free of bacteria. In preliminary studies, bacteria were sometimes seen swimming around during the course of a measurement that could last up to 6 hours. Bacteria stick to the droplet's surface or can become trapped in the same optical trap as the droplet this ends the experiment. Therefore emulsion storage conditions and sample preparations were critical.

To determine the ideal emulsion formulation to meet the criteria for performing our proposed direct force measurements a range of oil concentrations (10, 30 and 60 wt.\%) and emulsifier concentrations (1.0, 1.5 and 2.0 wt.\%) were tested under different homogenization conditions (34 MPa, $103 \mathrm{MPa}$ and $206 \mathrm{MPa}$ ) and with different continuous phases $(1,10,50$ and $100 \mathrm{mM}$ phosphate buffer at $\mathrm{pH} 7.0)$. As expected, each of these factors was found to affect the particle size and distribution.

\subsection{Emulsion design}

\subsubsection{Materials}

A phosphate buffer was prepared using sodium phosphate dibasic (Sigma-Aldrich, CAS 4558-794, $\mathrm{Na}_{2} \mathrm{HPO}_{4}, \mathrm{FW} 141.96 \mathrm{~g} / \mathrm{mol}$, d 1.52, hygroscopic) and monosodium Phosphate (Sigma-Aldrich, CAS 7558-8-7, $\mathrm{NaH}_{2} \mathrm{PO}_{4}, \mathrm{FW} 119.98$, Hygroscopic). $\beta$-lg kindly donated by Davisco Foods International, Inc. (Le Suer, Mn, USA; Lot \# JE001-0-415) with a $97.9 \%$ dry basis and a purity of $91.5 \%$. Sodium caseinate has $14.4 \%$ N and $1.4 \%$ sodium (Sigma-Aldrich, Product \# C8654, Batch \# 117K0138). 
Soybean oil (Sigma-Aldrich) was used to prepare all oil-in-water emulsions. The density of soybean oil is $0.917 \mathrm{~g} / \mathrm{mL}$ at $25 \mathrm{C}$ and the refractive index is 1.4743 according to the supplier. Sodium Azide (Sigma-Aldrich) was added to all emulsions prior to homogenisation at a concentration of $0.04 \mathrm{wt} . \%$ to inhibit bacterial growth. Deionised water (resistivity of $18.2 \mathrm{M} \Omega \mathrm{cm}$, Sartorius arium 611UV purification system) was used to make all solutions. For cleaning instruments, microfludic chips and other surfaces a industrial grade surfactant Decon 90 (Decon Laboratories Limited) was used.

\subsubsection{Emulsion Preparation}

Emulsions were prepared by first making an aqueous emulsifier solution by diluting $\beta$-lg or sodium caseinate at a concentration or 1.0, 2.0 or $3.0 \mathrm{wt} . \%$ in freshly prepared sodium phosphate buffer with an ionic strength (1-100 mM, pH 7.00). The protein was continuously stirred for two hours at room temperature until fully dissolved, and then stored at $4{ }^{\circ} \mathrm{C}$ overnight to ensure the protein was fully hydrated. A fine emulsion was produced using a bench-top high-pressure homogenizer (Avestin EmulsiFlex-C5) at pressures $34 \mathrm{MPa}$ to $206 \mathrm{MPa}$ for 1-5 passes. The emulsion was stored at $4{ }^{\circ} \mathrm{C}$ for 24 hours before any characterisation.

The Avestin EmulsiFlex-C5 homogenizer (Figure 4.10) was used to supply the energy required to break up the oil into discrete droplets to form a stable emulsion. This homogenizer has an air/gas driven pressure pump that can achieve pressures from 3.5-207 MPa (500-30000 psi). The holdback volume according to the manufacturer is less than $1 \mathrm{ml}$ providing minimal sample loss. The air supply was controlled by an air compressor (Auckland, NZ). The air compressor has a $50 \mathrm{~L}$ tank providing adequate air to achieve the pressure limits of the homogenizer. The tank pressure was set to $10 \mathrm{MPa}$ (450 psi), and the compressor maintained the pressure if the tank dropped below $9.5 \mathrm{MPa}(137 \mathrm{psi})$.

When working with a small sample size of less than $20 \mathrm{ml}$, the syringe attachments 


\section{Emulsion Introduction and Design}

were extraordinarily useful. As additional pressure could be supplied by pressing on the syringe to maintain an even pressure for each pass through the homogeniser.

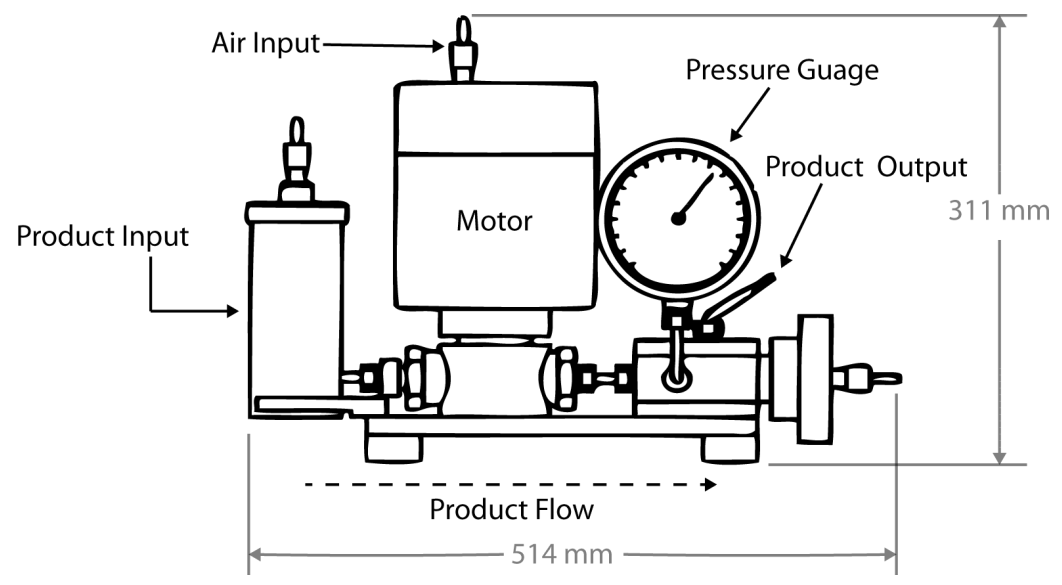

Figure 4.10.: Avestin EmulsiFlex-C5 high-pressure homogenizer.

\subsection{Characterisation Techniques}

Standard emulsion characterisation techniques were used to determine the stability, particle size distribution and the zeta-potential of the droplet's surface . Static light scattering was used to measure the droplet size distribution. Dynamic light scattering was not used due to the droplet size probed in these experiments. Dynamic light scattering (DLS) is capable of measuring droplets ranging from $0.3 \mathrm{~nm}$ to 6 $\mu \mathrm{m}$ and static light scattering (SLS) is a more appropriate choice for measuring droplets ranging from $0.2 \mathrm{~nm}-2000 \mu \mathrm{m}$ according to the manufacturer. The droplet size distribution was measured at 1 and 8 days after homogenisation to ensure the emulsion was stable over the course of one week. For the emulsion formulation used for direct force measurements the $\zeta$-potential was measured to determine the charge at the droplet's surface as this is an important measurable parameter of the interaction between droplets and understanding its distribution or homogeneity between droplets is an interesting study. 


\subsubsection{Droplet Size Distribution}

Particle size distributions of the emulsions were measured using a Mastersizer 2000 (Malvern, United Kingdom) static light scattering instrument(Figure 4.11). The diluted samples were pumped through a measurement cell where a red $(4 \mathrm{~mW}$ He-Ne, $632.8 \mathrm{~nm})$ and blue (0.3 $\mathrm{mW}$ LED, $470 \mathrm{~nm})$ light were passed through the sample. Two light sources at different wavelengths were used to improve the sub-micron particle sensitivity. When the laser light enters the sample cell light may pass through the sample, be absorbed or be scattered. The intensity of the scattered light is detected by a series of 52 detectors that are strategically placed from small $\left(0.02^{\circ}\right)$ to large angles $\left(135^{\circ}\right)$. The detectors are arranged in a logarithmic sequence where at small angles they are grouped closely together and are more widely spaced at large angles. Mie theory is used to convert the intensity distribution into a volume distribution using the Malvern Mastersizer 2000 program version 5.60. The experimental results are fitted to Mie Theory and the difference between the experimental data, and theoretical calculations validate the accuracy of the calculated particle size distribution. In Mie Theory, it is assumed that all particles are perfect

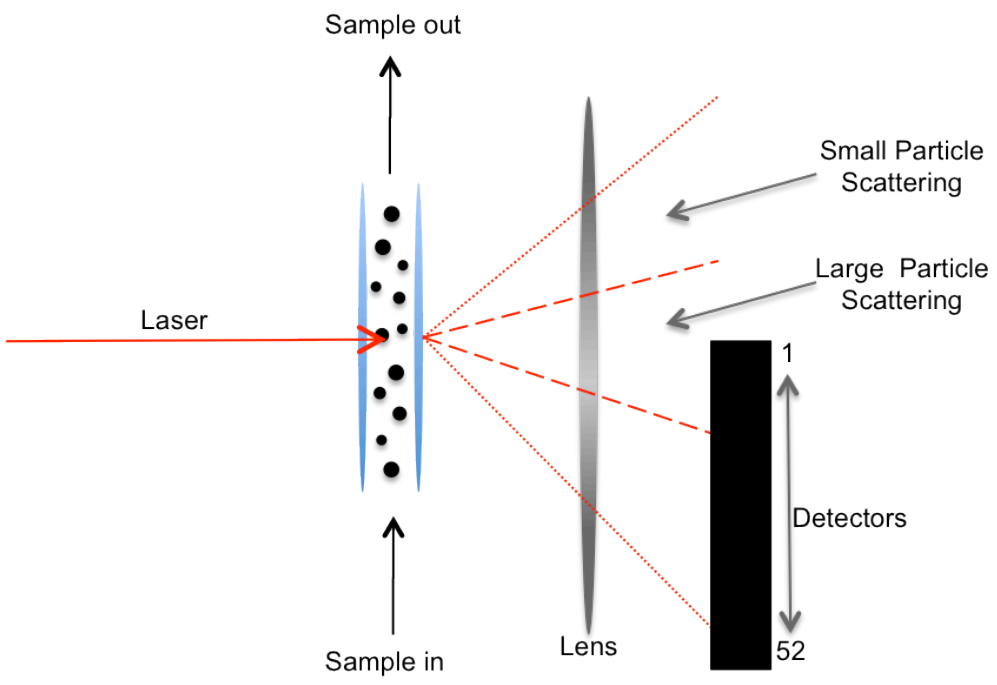

Figure 4.11.: Schematic of the MALVERN Mastersizer 2000, static light scattering instrument. 


\section{Emulsion Introduction and Design}

homogeneous spheres that have a different refractive index than the medium.

The light is assumed to undergo only a single scattering event. Therefore, the emulsion has to be diluted. Samples in this work were diluted into in $800 \mathrm{ml}$ of deionised water to an obscuration of 10-15\%. The sample was stirred at a speed of $2000 \mathrm{rpm}$ and before measurement the diluted sample underwent 30 seconds of sonication to break up loose flocks that may have formed and to ensure the droplets were evenly distributed. The refractive indices for the droplets and the dispersant were set to 1.47 and 1.33 respectively. Droplet size distributions are compared using the mean droplet diameter $d_{32}$ (Sauter mean diameter) and the Span. Span, also known as the dispersion index is defined as

$$
\text { Span }=\frac{d[90]-d[10]}{d[50]}
$$

where $d[x]$ is the average droplet size in volume under which $[x] \%$ of the total sample weight remains, respectively. Each measurement was carried out in triplicate, and each emulsion was measured in at least duplicate.

\subsection{2. $\zeta$-potential}

Most particles in an aqueous medium carry an electric charge. The charged particle surface affects the distribution of ions in the interfacial region, resulting in an increased concentration of counterions close to the particles' surface. This concentration of ions on the surface is broken into two layers, making up the electrical double layer. First, the concentration of particles closest to the particle surface is the Stern layer where the counter-ions have the strongest affinity. The second layer is the outer layer or the diffuse layer where the counter-ions are less firmly attached. When the particles move due to gravity or other forces, there is a boundary (slipping plane). The ions either move with the particle or are not affected by the particles' movement. An electrical potential exists between the particle surface, and the double layer, the 
potential at this boundary is the $\zeta$-potential. The $\zeta$-potential measures the charge on the particle and the magnitude of the charge is an indication of emulsion stability. A large negative or positive $\zeta$-potential results in strong electrostatic forces between droplets, which can yield a more stable emulsion. Although, it has been reported that a $\zeta$-potential greater than $+/-30 \mathrm{mV}$ results in a stable colloidal system. The $\zeta$-potential cannot be used solely to describe emulsion stability, as other factors must also be considered including particle size and the environment surrounding the droplet. A diagram of the electrical double layer is shown in Figure 4.12.

\subsubsection{Zeta-Potential Measurements}

The MALVERN Zetasizer NanoZS was used with the MALVERN Universal dip cell or a disposable capillary cuvette to measure the average $\zeta$-potential of the emulsion droplets used. The choice between the dip cell and the capillary cuvette was sample dependent. The difference between them is the distance between electrodes: the distance between the electrodes of the dip cell is less than the U-shaped capillary cuvette. The diluted sample was added to a disposable cuvette where a universal dip cell was inserted with the assurance of no air bubbles. A laser is split into a reference beam and a scattering beam that provides the intense light source, which is passed through the diluted sample where the attenuator adjusts the intensity of the laser light beam to optimize the laser detection of the scattering at $13^{\circ}$ and/or $90^{\circ}$. The laser is passed through the centre between the two electrodes of the dip-cell. An electric field is applied across the sample, and the charged particles are attracted toward the electrode of the opposite charge. The viscous forces acting on the particles oppose the electrostatic forces between the charged particle and electrode. When equilibrium is reached between the two opposing forces, the velocity of the charged particles becomes constant. The velocity of the particle is also known as the electrophoretic mobility, which is affected by the strength of the electric field, the dielectric constant of the medium, the viscosity of the medium and the $\zeta$-potential. 


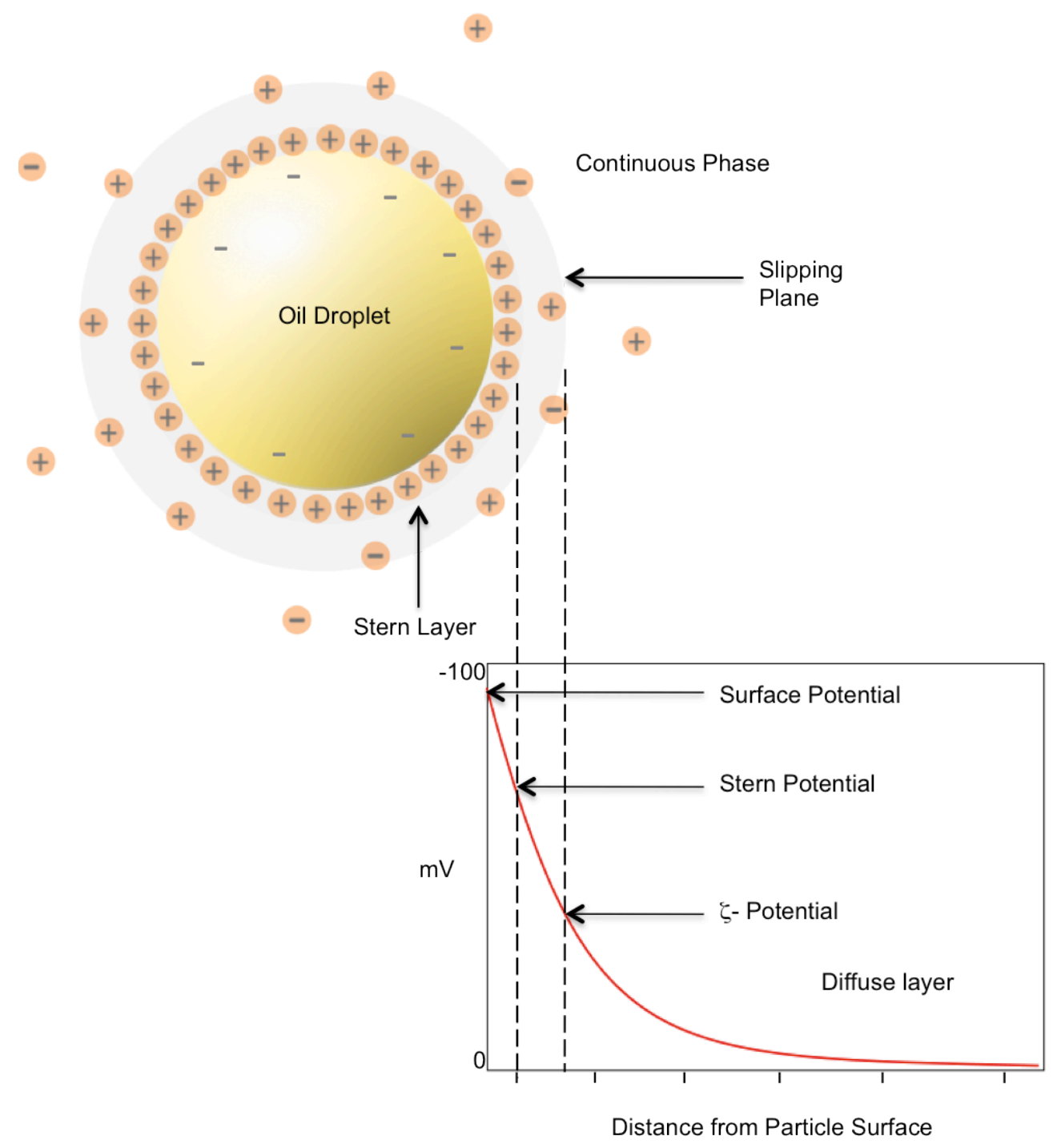

Figure 4.12.: Diagram of the electrical double layer of an emulsion droplet.

The light scattered at the detection angle is combined with the reference beam. This produces a fluctuating intensity signal where the rate of fluctuation is proportional to the speed of the particles. A digital processor is used to extract the characteristic of the scattered light. The MALVERN Zetasizer software then processes this information 
where the Zeta potential is calculated via Henry's Equation.

$$
U_{E}=\frac{e \epsilon \zeta}{3 \eta} F(\kappa a)
$$

Where $U_{E}$ is the electrophoretic mobility, $\epsilon$ is the dielectric constant of the medium, $\zeta$ is the $\zeta$-potential, $\eta$ is the viscosity of the medium, and $F(\kappa)$ is Henry's function. $\kappa a$ is the ratio of the particle radius to the electrical double layer thickness, where $\kappa^{-1}$ is the Debye length, and $a$ is the radius of the particle. $F(\kappa a)$ for our measurements is estimated to be 1.5 (this is referred to as the Smoluchowski approximation). The Smoluchowski model is used for particles larger than $0.2 \mu \mathrm{m}$ dispersed in electrolytes containing more than $10^{-3} \mathrm{M}$ for a monovalent salt.

\subsubsection{Microscopy}

Samples were characterised by cryo-scanning electron microscopy (cryo-SEM). CryoSEM images were acquired with a JEOL JSM-6500F field emission SEM fitted with a Gatan Alto 2500 cryo attachment. The samples were mounted on a sample holder (sandwiched between two copper plates) and plunged into liquid nitrogen 'slush' under vacuum. The sample holder was then transferred under vacuum to the cryo-preparation chamber, which was at $-130{ }^{\circ} \mathrm{C}$. The copper plates were fractured apart with a scalpel exposing the sample. The sample was then sublimated at -90 ${ }^{\circ} \mathrm{C}$ for approximately 5 minutes. Two coats of platinum were applied within the preparation chamber, and the sample was transferred to the SEM chamber. An accelerating voltage of $16.00 \mathrm{kV}$ and a probe current of $9.0 \mu \mathrm{A}$ were typically used. The instrument was operated at a working distance of approximately $10 \mathrm{~mm}$. 


\section{Emulsion Introduction and Design}

\subsection{Results and discussion}

Designing an emulsion that successfully met the criteria for direct force measurements was challenging. The most difficult criteria were producing an emulsion with a low polydispersity with a mean droplet size of approximately $2 \mu \mathrm{m}$ using highpressure homogenisation. In order to achieve this criteria, both formulation and processing conditions were considered. Two emulsifiers $\beta$-lg and sodium caseinate were investigated. All emulsions were prepared the day before characterisation.

\subsubsection{Effect of Homogenisation Pressure}

Many studies have investigated the effect of high-pressure homogenisation on droplet size distribution ${ }^{70,89-91}$. This is because shelf-life and texture of the emulsion significantly depend on the droplet size distribution which can be tuned by controlling the rate of droplet breakage and coalescence during emulsion formation ${ }^{70}$. However, very few studies have focused on understanding emulsion formation and formulation to a degree that allows for particular droplet distributions to be probed using highpressure homogenisation, especially with proteins at the interface. The reason this has yet to be solved is that there are many parameters that affect droplet size (i.e. oil phase, emulsifier, buffer conditions, etc.) which are all interrelated. Consequently, there is more than one pathway that will yield a desirable droplet size distribution to meet the criteria for direct force measurement.

In this study, high-pressure homogenisation was chosen over other emulsification methods because it is the most common method used in the food industry to prepare emulsions. The processing parameters that affect the droplet distribution includes pressure, number of passes and temperature. It should be noted that additional techniques have been developed to produce emulsions approaching monodispersity using droplet generator microfluidic chips, however, they have low throughput and although not impossible to achieve $2 \mu \mathrm{m}$ droplets is pushing the boundaries of the 
technique.
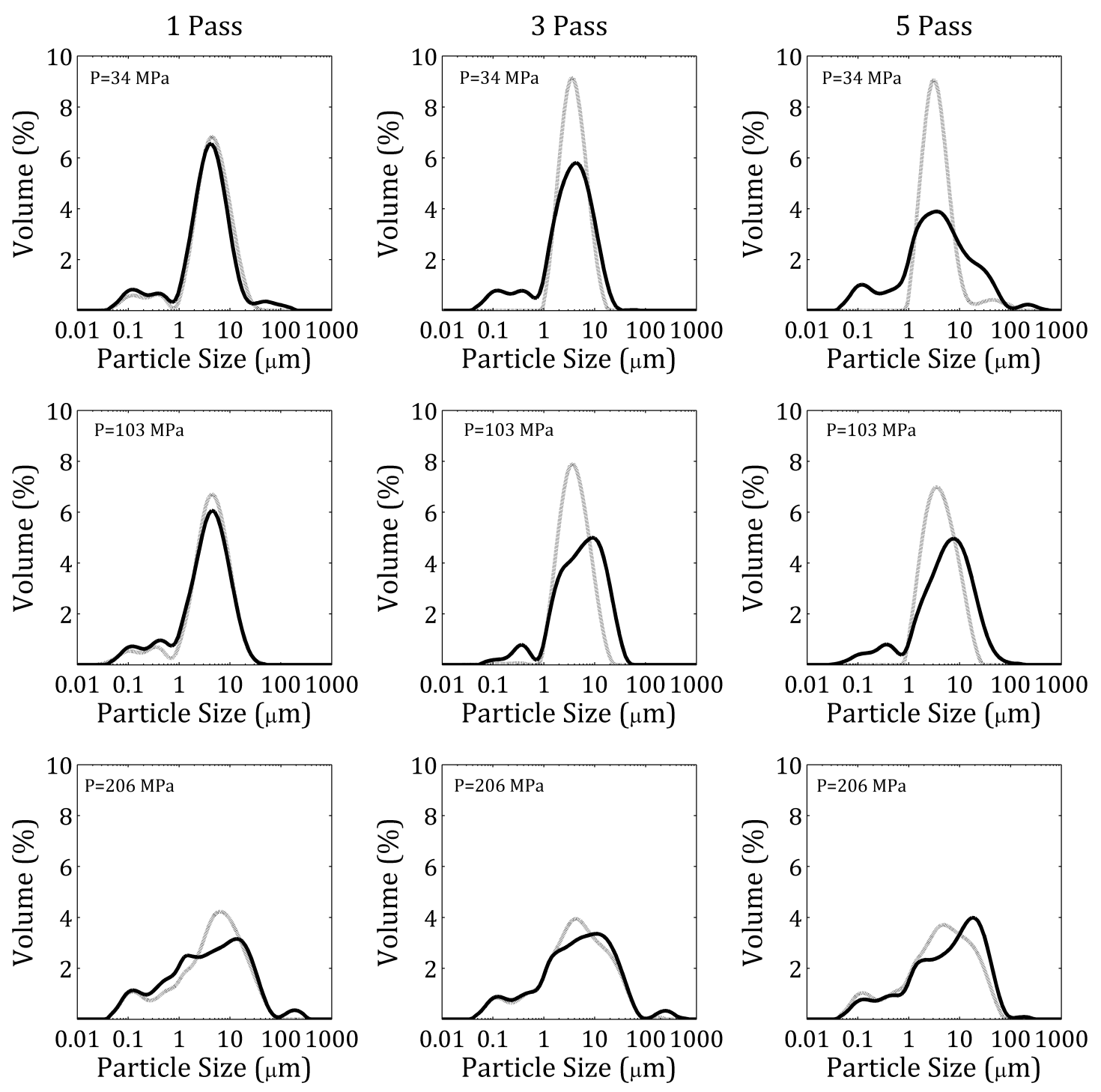

Figure 4.13.: Particle size distribution of sodium caseinate (grey) and $\beta$-lg stabilised emulsion (black) homogenised at different conditions. An increase in homogenisation pressure from $34 \mathrm{MPa}, 103 \mathrm{MPa}$ and $206 \mathrm{MPa}$ is represented in rows 1-3. Samples were collected at 1, 3 and 5 passes, and this is represented by columns 1-3, respectively.

Figure 4.13 shows a comparison between sodium caseinate and $\beta$-lg stabilised emulsions prepared at 34, 103 and $206 \mathrm{MPa}$. Samples were collected at 1, 3 and 5 passes. The emulsions contained 60 wt.\% soybean oil, 1 wt.\% protein in $100 \mathrm{mM}$, pH 7.0 


\section{Emulsion Introduction and Design}

phosphate buffer as the aqueous phase. $\beta$-lg produced a more polydisperse emulsion at almost all pressures and number of passes investigated. At one pass through the homogeniser, $\beta$-lg and sodium caseinate resulted in similar distributions, however, as the number of passes increased the differences between the distributions became more evident. This difference is attributed to the structural differences between the proteins and their ability to satisfy the interface.

At pressure $206 \mathrm{MPa}$ the emulsions had the greatest polydispersity. Table 4.4 shows that the span of the emulsions produced at this high pressure increased almost by a factor of 2 compared to samples prepared at lower pressures. This is also represented in Figure 4.13 where the bottom row shows a broad polydispersity compared with lower homogenisation pressures. This wide distribution suggests the emulsions were 'over-processed' reducing the emulsification capacity of the protein. This is a well known phenomena first reported by Tornberg in $1980^{92}$. An additional test was performed to rule out that it could have been an effect of protein concentration, for example, by not providing enough protein to satisfy the smallest droplets. However, increasing the protein concentration to $2 \mathrm{wt} . \%$ made no difference, and the distribution was similarly broad (data not shown).

When comparing the number of passes through the homogeniser at pressure $34 \mathrm{MPa}$, it was found that both $\beta$-lg and sodium caseinate emulsions are 'overprocessed' at 5 passes indicated by the wider distribution. At five passes, the $\beta$-lg stabilised emulsion shows a span of 7.53 as shown in Table 4.4, which in fact was the largest span out of all the emulsions investigated. Although, the sodium caseinate was still able to maintain its integrity after five passes, aggregates were observed above $10 \mu \mathrm{m}$.

$\beta$-lg is more sensitive to structural changes during high-pressure processing than sodium caseinate. Globular, tertiary more compact proteins like $\beta$-lg can undergo significant structural modifications at pressures greater than $200 \mathrm{MPa}^{93}$. Denaturation occurs at different combinations of pressure and temperature. $\beta$-lg processed over 200 MPa has significant aggregation that negativity influences the emulsification of the protein onto the oil/water interface ${ }^{93}$. According to Galazka et al. the aggregation is 
Table 4.4.: Average droplet size distributions of sodium caseinate and $\beta$-lg emulsion stabilised oil-in-water emulsions. Standard deviations of 6 measurements are represented in parentheses. Presented are the average $d_{3,2}$ diameter of droplets along with the span. An increase in the polydispersity of the emulsion increases the span number. A monodispersed emulsion will have a span value close to 1.0 .

\begin{tabular}{cc|cc|cc|cc|cc}
\hline & & \multicolumn{3}{|c|}{ Sodium Caseinate } & \multicolumn{3}{c|}{$\beta$-lactoglobulin } \\
\hline $\begin{array}{c}\text { Pressure } \\
(\text { MPa) }\end{array}$ & $\begin{array}{c}\text { Passes } \\
\#\end{array}$ & \multicolumn{2}{|c|}{$\begin{array}{c}\boldsymbol{d} \text { [3,2] } \\
(\mu \mathrm{m})\end{array}$} & \multicolumn{2}{c|}{ Span } & \multicolumn{2}{c}{$\begin{array}{c}\boldsymbol{d} \text { [3,2] } \\
(\mu \mathrm{m})\end{array}$} & \multicolumn{2}{|c}{ Span } \\
\hline \multirow{3}{*}{34} & 1 & 1.13 & $(0.22)$ & 2.42 & $(0.03)$ & 0.87 & $(0.08)$ & 2.91 & $(0.34)$ \\
& 3 & 3.42 & $(0.51)$ & 1.67 & $(0.03)$ & 0.84 & $(0.04)$ & 2.84 & $(0.07)$ \\
& 5 & 2.80 & $(0.05)$ & 2.04 & $(0.33)$ & 0.72 & $(0.05)$ & 7.53 & $(1.00)$ \\
\hline \multirow{2}{*}{103} & 1 & 1.09 & $(0.06)$ & 2.55 & $(0.08)$ & 0.89 & $(0.19)$ & 2.73 & $(0.15)$ \\
& 3 & 3.21 & $(0.75)$ & 1.97 & $(0.32)$ & 1.77 & $(0.21)$ & 2.97 & $(0.08)$ \\
& 5 & 3.62 & $(0.29)$ & 2.26 & $(0.01)$ & 1.22 & $(0.02)$ & 3.43 & $(0.21)$ \\
\hline \multirow{2}{*}{206} & 1 & 0.80 & $(0.14)$ & 3.80 & $(0.33)$ & 0.63 & $(0.01)$ & 6.97 & $(0.67)$ \\
& 3 & 0.71 & $(0.14)$ & 5.49 & $(0.14)$ & 0.84 & $(0.09)$ & 5.82 & $(0.41)$ \\
& 5 & 0.72 & $(0.04)$ & 5.35 & $(0.13)$ & 0.92 & $(0.07)$ & 4.71 & $(0.13)$ \\
\hline
\end{tabular}

most likely resulting from the formation of intermolecular disulfide bridges between the partially denatured proteins ${ }^{94}$.

Galazka et al. showed that if $\beta$-lg solutions were pressure treated prior to homogenisation, the droplets were substantially larger compared to an emulsion prepared with the native protein. This result indicates that the modification made to the protein via pressure lead to the loss of emulsifying efficiency due to the aggregated state of the proteins in solution. Thus, the growth of the droplet size distribution at $206 \mathrm{MPa}$ presented in Figure 4.13 is most likely caused by protein modification and aggregation at high pressures. 


\section{Emulsion Introduction and Design}

\subsubsection{Effect on Homogenisation Temperature}

The mechanical energy applied to force an emulsion through a tiny orifice resulting in discrete droplets is immense ${ }^{70,90}$. During this process, the mechanical energy is dissipated into the emulsion in the form of heat, increasing the temperature of the emulsion. This temperature increase is directly proportional to the pressure ${ }^{70,90}$. Floury et al. and Desrumaux, and Marcand studied this effect and showed that the temperature increased from $25^{\circ} \mathrm{C}$ to $65^{\circ} \mathrm{C}$ from pressures of $25 \mathrm{MPa}$ to $300 \mathrm{MPa}$ in their respective studies ${ }^{70,90}$. In these studies, they used cooling jackets containing chilled water at $5{ }^{\circ} \mathrm{C}$ around the output of the emulsion to help reduce the effect of temperature $^{70,90}$. However, this was not used for this thesis. The exit temperatures measured in this study were slightly higher because a cooling jacket was not used. The temperature of the coarse emulsion prior to homogenisation is approximately 32 $\pm 2{ }^{\circ} \mathrm{C}$. The emulsion temperature post homogenisation is $47 \pm 3{ }^{\circ} \mathrm{C}$ at $103 \mathrm{MPa}$. The temperature reported in literature at the same pressure was approximately 35 ${ }^{\circ} C^{70}$ and $18{ }^{\circ} C^{90}$. The temperature was also monitored from 1-3 passes. However, the emulsion exit temperature only increased a few degrees (data not shown).

The temperatures reported in the literature at $200 \mathrm{MPa}$ are close to the denaturation temperature of the protein. According to literature the exit temperature from the homogeniser is reported be $50-55^{\circ} \mathrm{C}^{70,90}$. At this temperature, both proteins will have experienced structural transitions. Bovine casein has four distinct structural transitions at $10,33,40$ and $78^{\circ} \mathrm{C}$ at $\mathrm{pH} 6.75$, these temperatures were determined by circular dichroism analysis ${ }^{95}$. Qi et al. attributed $10^{\circ} \mathrm{C}$ to a cold conformational change where 33 and $40^{\circ} \mathrm{C}$ more likely reflect of hydrophobic changes in the core of beta-casein ${ }^{95}$. When the temperature is increased to $78^{\circ} \mathrm{C}$, the beta-casein undergoes self-association into more intricate aggregated formations ${ }^{95}$. Similarly, $\beta$-lg will also experience structural transitions at $35-45^{\circ} \mathrm{C}$ and greater aggregation due to the proposed disulfide bond formation at $60{ }^{\circ} C^{95}$. The aggregation formed by an increase in temperature is described by the same mechanism as an increase in pressure supporting the effect of 'overprocessing' at high pressures. 
The emulsion formulations with the lowest polydispersity were the sodium caseinate emulsions at $34 \mathrm{MPa}$ and $103 \mathrm{MPa}$ at 3 passes. These emulsions met the criteria for direct force measurements. The droplet distribution of these emulsions were measured again at 8 days to determine if the emulsion possessed any instabilities. Figure 4.14 shows that the sodium caseinate emulsion at $34 \mathrm{MPa}$ indicated some instability as a population of droplets or aggregates above $10 \mu \mathrm{m}$ were observed. At $103 \mathrm{MPa}$, the droplet distribution showed no indication of instabilities. $\beta$-lg emulsions were also measured again after 8 days at 34 and $103 \mathrm{MPa}$. The $\beta$-lg emulsions did not show any evidence regarding instabilities at both pressures, however, at $103 \mathrm{MPa}$ the emulsion droplet distribution reduced from a $d_{3,2}$ value of $1.77 \mu \mathrm{m}$ to $1.13 \mu \mathrm{m}$ without significantly changing the shape of the distribution curve. This reduction in droplet size could be attributed to a viscosity effect. The $\beta$-lg emulsion homogenised at 103 $\mathrm{MPa}$ was very thick like greek yoghurt in comparison to more cream like emulsions. Since this emulsion was not a good candidate for direct force measurements, this was not investigated further.

\subsubsection{Effect of Protein Concentration}

It is well known that the ratio between the amount of protein and oil strongly affects the mean droplet size. Increasing the protein concentration allows for more protein to be available during homogenisation to coat the newly formed oil droplets and prevent re-coalescence. Smaller droplets have greater surface area than the larger ones requiring additional protein.

Figure 4.15 shows the effect of protein concentration on droplet size. A series of emulsions were prepared increasing the protein concentration from 1-2 wt.\% protein in the aqueous phase, while maintaining the oil concentration at 60 wt.\% and homogenisation conditions (103 MPa, 3 passes).

As expected, Figure 4.15 shows that increasing the protein concentration for both sodium caseinate and $\beta$-lg from 1-2 wt.\% in the aqueous phase of the emulsion 


\section{Emulsion Introduction and Design}
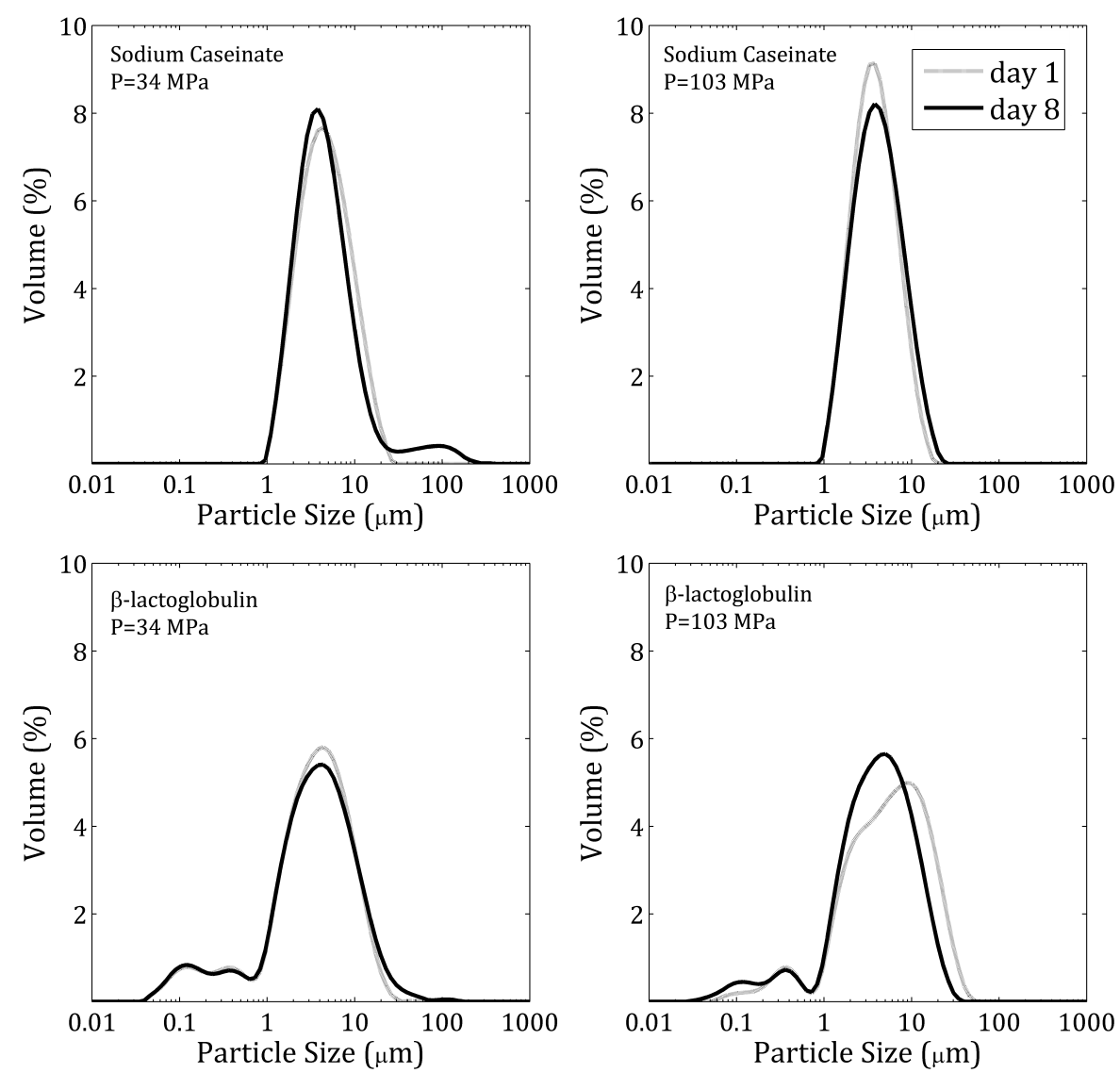

Figure 4.14.: Particle size distribution of sodium caseinate (top row) and $\beta$-lg stabilised emulsion (bottom row) homogenised at $34 \mathrm{MPa}$ (left column) and $103 \mathrm{MPa}$ (right column) for 3 passes.

decreased the droplet size distribution. Interestingly, the decrease in the $d_{3,2}$ value from 1-2 wt.\% was similar at $1.14 \mu \mathrm{m}$ and $1.19 \mu \mathrm{m}$ for sodium caseinate and $\beta$ $\lg$ respectively. This indicates that the ratio between the oil and the emulsifier is correlated to droplet size distribution, regardless of the protein used in the formulation. 

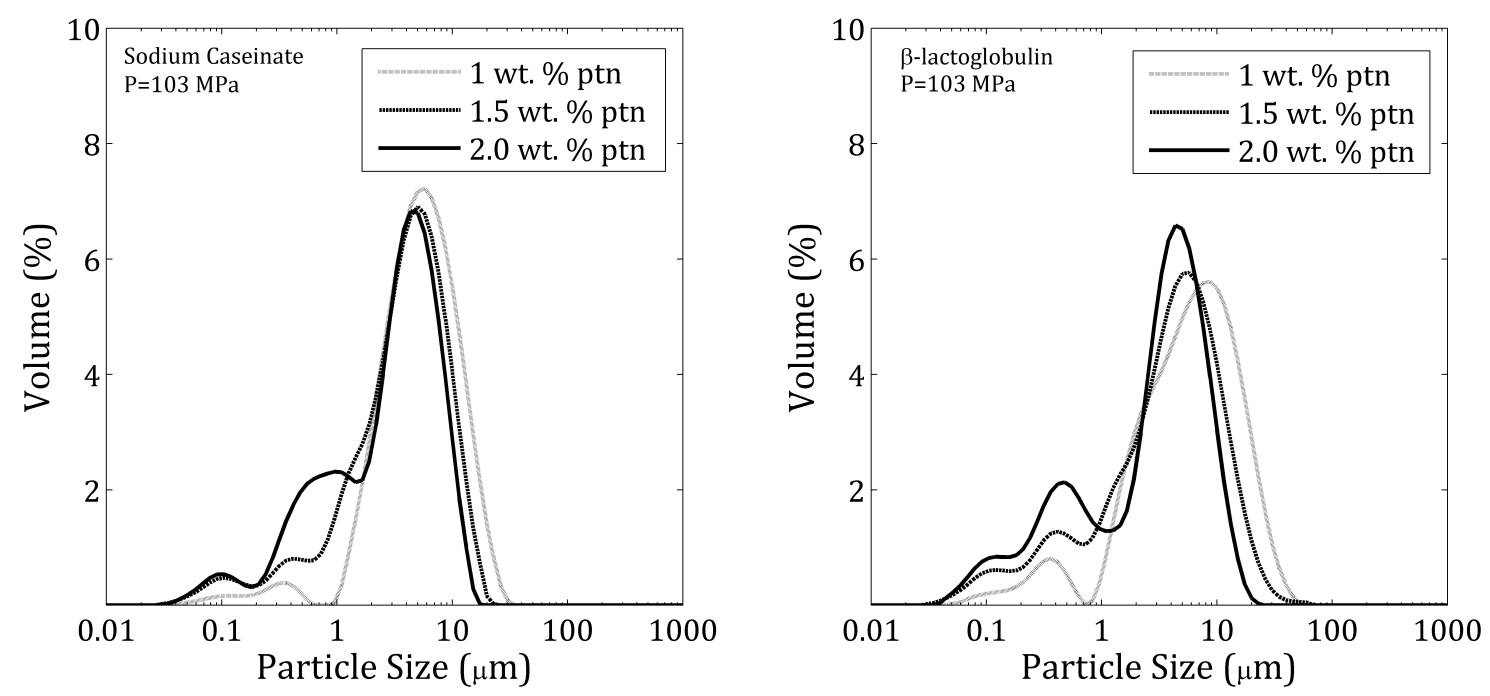

Figure 4.15.: Comparison between sodium caseinate and $\beta$-lg stabilised emulsions at protein concentrations in the aqueous phase of $1,1.5$ and $2 \mathrm{wt} . \%$ protein, $60 \mathrm{wt} . \%$ oil, $100 \mathrm{mM}$ phosphate buffer at $\mathrm{pH}$ 7.0. The emulsion was prepared at $103 \mathrm{MPa}$ for 3 passes through the homogeniser. The $d_{3,2}$ values decreased with an increase in protein concentration.

\subsubsection{Effect of Oil Concentration}

Similarly, by decreasing the oil concentration from 60 wt. $\%$ to 10 wt $\%$ and maintaining the protein concentration at $1 \mathrm{wt} . \%$ in the continuous phase, the droplet size also decreases. This is shown to reiterate that altering this ratio between emulsifier and oil can substantially change the droplet size distributions. Sodium caseinate, the left plot in Figure 4.16 shows a change in the protein concentration from 60 wt.\% to 10 wt. $\%$ decreasing the particle size from a $d_{3,2}$ of $4.30 \mu \mathrm{m}$ to $220 \mathrm{~nm}$ respectively. $\beta$-lg showed a similar decrease between 60 wt. $\%$ oil and 10 wt.\% oil were the $d_{3,2}$ of $2.09 \mu \mathrm{m}$ to $200 \mathrm{~nm}$. However, the difference between $60 \mathrm{wt} . \%$ oil and $30 \mathrm{wt} . \%$ oil is less clear. 30 wt.\% oil has measured a large particle at $100 \mu \mathrm{m}$. This is most likely not a part of the actual emulsion but an artefact of an air bubble or dust. 

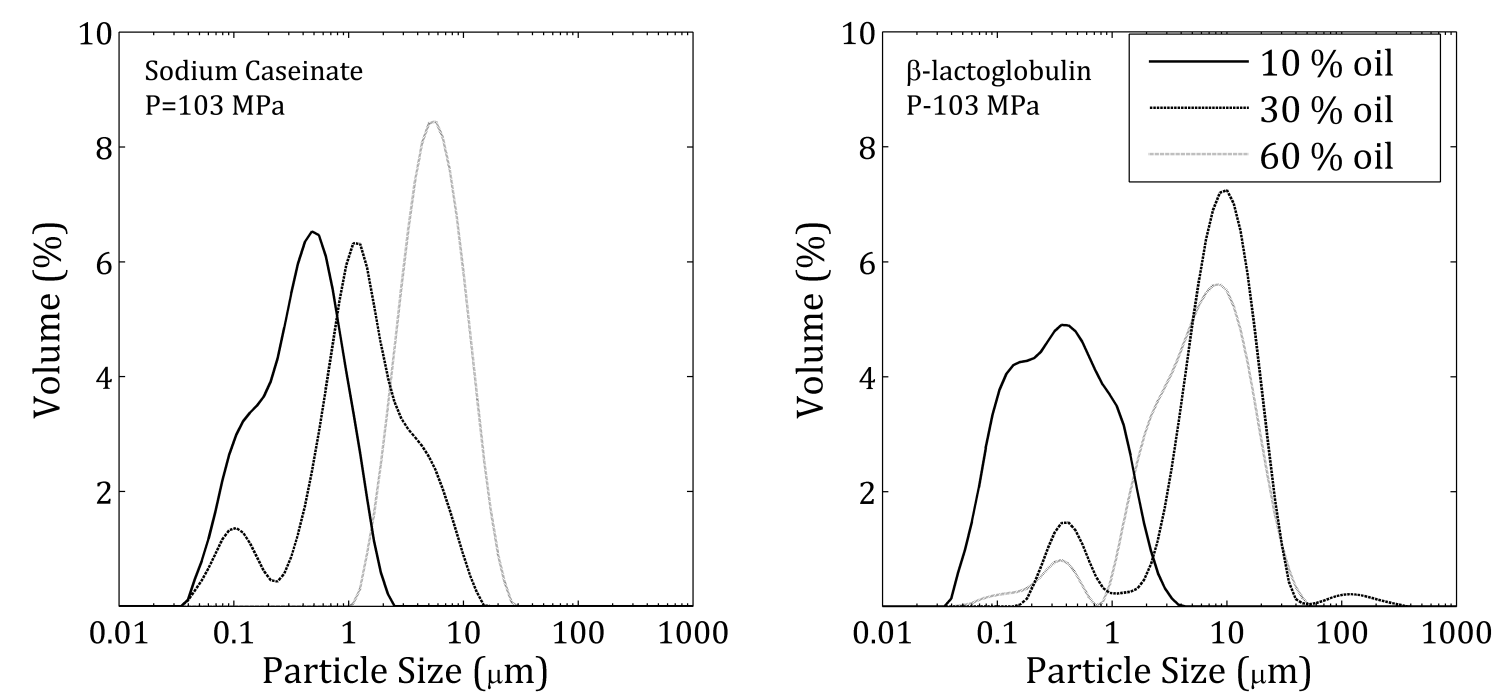

Figure 4.16.: Comparison between sodium caseinate (left plot) and $\beta$-lg (right plot) stabilised emulsions at protein concentrations of $10 \mathrm{wt} . \%, 30 \mathrm{wt} . \%$ and $60 \mathrm{wt} . \%$ oil in a phosphate buffer of $100 \mathrm{mM}, \mathrm{pH} 7.0$.

\subsubsection{Effect of lonic Strength}

It is well known that ionic strength affects the effective charge on the protein as well as the conformational state of a protein on the interface. These changes in conformation in turn change the steric stabilisation which is dependent on the structural and molecular make-up of the protein. Previously discussed in Section 4.3, $\beta-\lg$ and sodium caseinate have different structural components yielding different interfacial behaviour. Figure 4.17 demonstrates how the change in ionic strength from of the continuous phase $1-100 \mathrm{mM}$ effects droplet size. $\beta$-lg and sodium caseinate emulsions were prepared at $60 \mathrm{wt} . \%$ oil, 1 wt. $\%$ protein in the aqueous phase at pH 7.0, under the same homogenisation conditions (103 MPa, 3 passes). Sodium caseinate and $\beta-\lg$ show similar behaviour at ionic strengths of 1-10 $\mathrm{mM}$, however, at ionic strengths above $50 \mathrm{mM}$ sodium caseinate emulsions have a lower polydispersity than the equivalent $\beta$-lg emulsion.

The transition from a broad distribution at ionic strengths of 1-10 $\mathrm{mM}$ to a more 

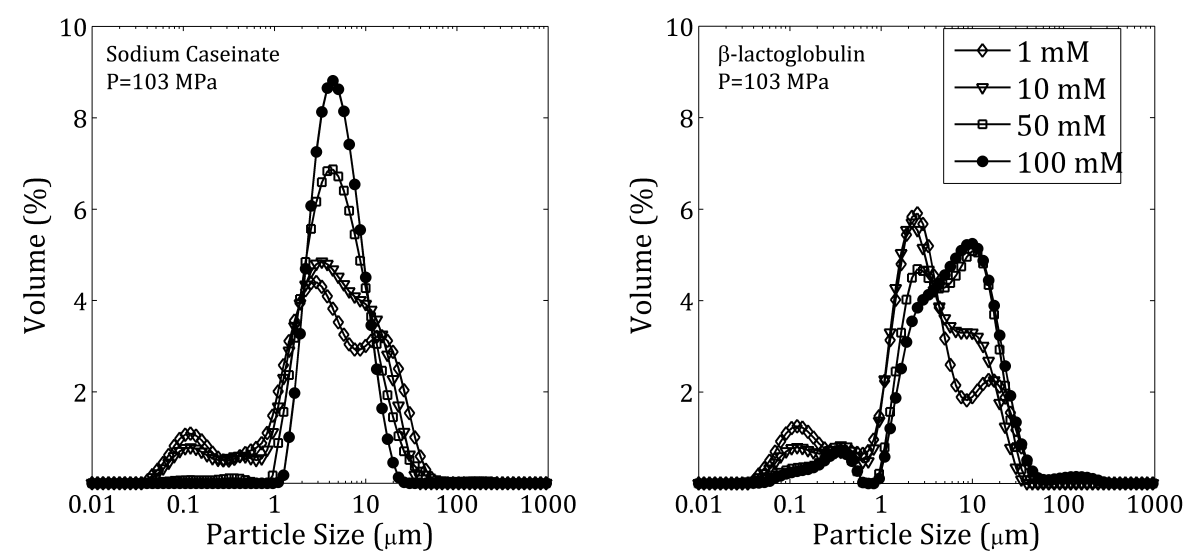

Figure 4.17.: Comparison between sodium caseinate (left plot) and $\beta$-lg (right plot) stabilised emulsions at ionic strengths of $1 \mathrm{mM}, 10 \mathrm{mM} 50 \mathrm{mM}$ and $100 \mathrm{mM}$ at pH 7.0.

monomodal polydispersity from 50-100 $\mathrm{mM}$ may be due to the aggregation state of the protein. The increase in ionic strength reduces the emulsification capacity of the protein reducing the absorption during and after homogenisation. Although, both $\beta$-lg and sodium caseinate showed an increase in droplet size sodium caseinate achieved the monomodal distribution desired.

In respect to sodium caseinate when salt is present, the proteins are known to aggregate as their effective charge is reduced. During emulsion formation, the protein was hydrated with the salt present in solution and allowed to come to an equilibrium overnight. The protein solution was then used to prepare the emulsion. The protein will have a different aggregation and conformational state at $100 \mathrm{mM}$ than at 1 $\mathrm{mM}$. At low salt concentrations the protein has more affinity to satisfy the oil-water interface that is evident by the population of droplets at $100 \mathrm{~nm}$.

\subsubsection{Droplet $\zeta$-potential}

The droplet's surface charge is related to the ionic environment. The surface charge is a very important parameter which will dictate the electrostatic interactions discussed in the following chapter. The surface charge on the droplets is related to the 

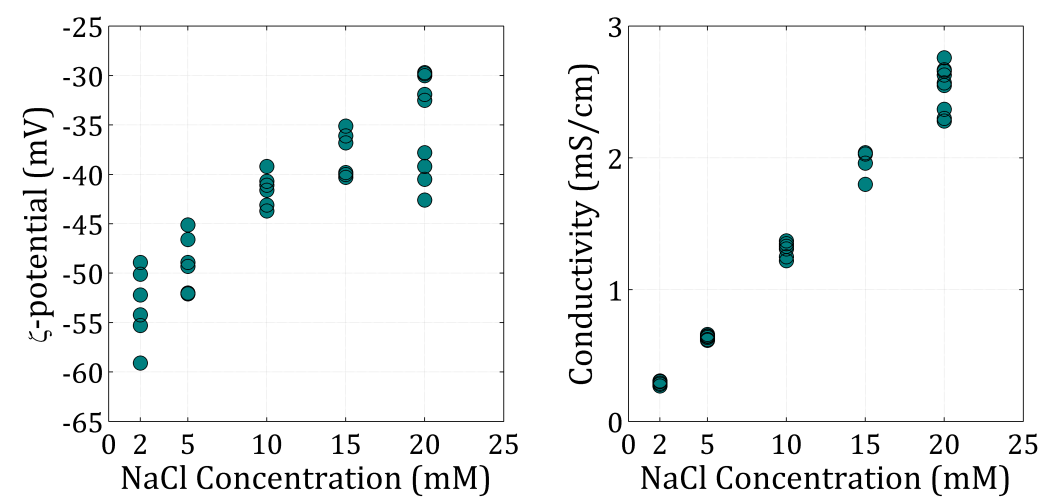

Figure 4.18.: The $\zeta$-potential of sodium caseinate emulsions at increasing ionic strength at neutral $\mathrm{pH}$. The right plot shows the corresponding conductivity of the continuous phase.

measurable $\zeta$-potential which was introduced as a technique in Section 4.7.2. Figure 4.18 shows the change in $\zeta$-potential at $\mathrm{NaCl}$ concentrations from $1-20 \mathrm{mM}$. It was expected that an increase in the electrolyte in solution would decrease the $\zeta$-potential. It is known that for monovalent salts the $\zeta$-potential increases linearly. $\mathrm{NaCl}$ concentrations less than $1 \mathrm{mM}$ were not able to be measured because there are not enough ions to move the droplets from one electrode to the other during the measurement.

\subsection{Conclusion}

It was determined that sodium caseinate produced a more stable emulsion at low polydispersity than the $\beta$-lg emulsion. The final formulation for the emulsion used in the direct force measurements is 60 wt.\% oil, 1 wt.\% sodium caseinate in an aqueous phase of $100 \mathrm{mM}$ phosphate buffer at $\mathrm{pH}$ 7.0. The homogenisation conditions to prepare this emulsion were $103 \mathrm{MPa}$ at 3 passes. This formulation met the requirements discussed in Section 4.5.1.

What made this a unique challenge is that this study required a specific droplet size whereas there was less of a concern about the overall physical properties of the 


\subsection{Conclusion}

emulsion. Due to the nature of this research work the emulsion created was supposed to provide a 'best case scenario' where the measurements could be performed rather routinely. The technique for direct force measurements presented in this thesis can be utilised for a wide variety of emulsions, not just the formulation presented in this chapter. 


\section{Emulsion droplet interactions}

In this chapter the utility and future potential of the dual-trap optical tweezers method presented in Chapter 3 is demonstrated, by applying the method to a less homogeneous; system, protein coated emulsion droplets discussed in Chapter 4.5. Here, droplet-droplet interactions were measured as a function of droplet separation and compared to predictions based on the electrostatic component of the DLVO Theory.

\subsection{Introduction}

Emulsions are thermodynamically unstable making their stability and destabilization processes interesting both theoretically as well as in practice. Many destabilization processes in emulsions such as flocculation and coalescence are largely controlled by interparticle forces. However, there are few studies focused on directly measuring interactions between emulsion droplets and there is a lack of understanding both on how interactions between particular pairs of droplets might vary throughout the ensemble, and how these interactions generate the bulk properties of emulsions. This lack of understanding has been due at least in part to the lack of tools/equipment available to measure such properties in heterogeneous systems. 
Only a few investigations have been carried out on the trapping and manipulation of emulsion droplets using OTs. Ward et al. showed that it is possible to deform oil droplets with low surface tension using optical traps. Most interestingly they discovered that using a high laser power and a low surface tension, oil droplets could be formed into a dumbbell shape and stretched into two droplets tethered by a strand of oil. Upon bringing the two droplets back together there was no barrier to coalescence $^{96}$. More relevant to the work reported here Bauer et al. studied the $\mathrm{pH}$ dependent interactions of individual droplets using OTs ${ }^{97}$. Although, interactions were probed, there were no force curves presented in that work. Most recently, during the course of this thesis Nilsen-Nygaard et al. have shown that it is possible to measure interactions between emulsion droplets ${ }^{50}$. However, neither of these studies have brought a quantitative understanding to the presented force curve. In this Chapter I demonstrate that quantitative interactions can be measured, and physical meaning can be extracted from the force-displacement curves.

\subsection{Method}

Droplet-droplet interactions were measured between sodium caseinate stabilised soybean oil-in-water emulsion droplets. Concentrated emulsions were prepared at 60 wt.\% oil, 1 wt.\% sodium caseinate in $100 \mathrm{mM} \mathrm{pH} 7.0$ sodium phosphate buffer. The emulsion was pre-emulsified using a hand held mixer and then homogenised at 103 MPa for 3 passes. More details of the emulsion preparation can be found in Section 4.6.1. The emulsions had a droplet size $D[3,2]=3.2 \pm 0.8 \mu \mathrm{m}$ as measured by static light scattering. Emulsions were stored at $4{ }^{\circ} \mathrm{C}$ and were kept no longer than 1 week before experiments were performed. For direct force measurements the emulsions were diluted more than 1 million times (approximately by a factor of $10^{8}$ ) in water. This dilution results in a negligible amount of salt in the diluted solution.

Direct force measurements were carried out in the same manner as was discussed in Chapter 3. Two similar-sized droplets were first trapped in separate optical traps. 


\section{Emulsion droplet interactions}

Holograms were generated to step one droplet towards the other stationary droplet until displacement of the stationary droplet was visible. The bead movement was recorded at $250 \mathrm{~s}^{-1}$, with an exposure time of $2 \mathrm{~ms}$ and 60,000 frames were collected for each interaction. The droplet positions were determined by 3D-particle tracking as described in Chapter 2 and the submitted paper by Raudsepp et al. (Appendix C) $)^{54}$.

\subsection{Results}

\subsubsection{Determining Droplet Size}

To measure the interactions between colloidal particles, such as emulsion droplets of an unknown size it is important to experimentally determine this size so that surface to surface separation of the droplets can be obtained. In this study droplet size was determined by measuring the restricted diffusion of the droplet in a weak SLM generated optical trap.

A droplet was trapped in a low power SLM trap $(0.2 \mathrm{~W})$ and the movement of the droplet was filmed $(3 \times 5000$ frames per bead, $500 \mathrm{~Hz}$, exposure time $1 \mathrm{~ms})$. The region of interest (ROI) analysed was chosen so that the droplet's Brownian excursions remained inside the selected area, which resulted in a $80 \times 80$ pixel area with our $60 \times$ objective. Due to the reduced exposure time the background intensity was reduced to $\langle I\rangle \simeq 10$ kcounts. Corresponding reference images and background images were collected at the same exposure time for particle tracking. The droplet's position was determined by 3D particle tracking as described in Chapter 2 .

The MSD was calculated using Equation 5.1,

$$
m s d=\left\langle\left(u(t)-u_{0}^{2}\right)\right\rangle=2 D d t
$$


where $u=x, y$ or $z$ and $D$ is the diffusion constant of a bead with a radius $a$ in a Newtonian medium with a viscosity $\eta$, defined by $D=k_{b} T / 6 \pi \eta a^{54}$.

To demonstrate this method the hydrodynamic radius of silica beads of known size were measured ( $2 r=1.85 \mu \mathrm{m}, \mathrm{CV} 10-15 \%)$. Figure 5.1 (a) shows the uncalibrated MSD $\left(\right.$ pixel $\left.^{2}\right)$ of the averaged positions $(3 \times 5000$ frames $)$. Figure 5.1 (b) shows the calibrated MSD, assuming calibration factors $x c=y c=72 \mathrm{~nm} /$ pix and $z c=50$ $\mathrm{nm} /$ pix. The calibration factor in the $z$-direction was chosen so that the short time $z$ MSD (green) mapped onto the short time $x$ and $y$ MSD (blue and red). The expected diffusion constant of the beads in water at room temperature is $2.13 \times 10^{-13}$ $\mathrm{m}^{2} / \mathrm{s}$, based on the given bead size, and the viscosity of water $\left(1 \times 10^{-3} \mathrm{~kg} / \mathrm{m} \mathrm{s}\right)$. Finally, a model calculation (in black) of the restricted diffusion of such a bead in an optical trap at a diffusion constant of $2.13 \times 10^{-13} \mathrm{~m}^{2} / \mathrm{s}$ is shown in Figure 5.1 (c). Raudsepp et al. presents the model calculation where the motional averaging over the exposure time on the long time low frequency statistics and apparent trap strength is considered (Appendix C) ${ }^{54}$.

The diffusion constant based on the expected bead size of $2 r=1.85 \mu \mathrm{m}$ shows excellent agreement between the model calculation and the measured data. This agreement confirms that the measured particle size is a good estimate of the droplet's radius. The trap strengths required to achieve this agreement are $0.26 \mathrm{pN} / \mu \mathrm{m}$ in the $x$ - and $y$-directions and $0.14 \mathrm{pN} / \mu \mathrm{m}$ in the $z$-direction.

The same method was applied to emulsion droplets of an unknown size. Here, the diffusion constant was chosen based on how well the model calculation agreed with the experimentally measured MSD. Figure 5.2 shows the MSD for two different droplets. The left and right columns shows the uncalibrated MSD, the calibrated MSD and the MSD fit, for two beads of different sizes. The left column resulted in a diffusion constant of $1.2 \times 10^{-13} \mathrm{~m}^{2} / \mathrm{s}$ resulting in a radius of $1.80 \mu \mathrm{m}$ and the right column resulted in a diffusion constant of $0.7 \mathrm{~m}^{2} / \mathrm{s}$ resulting in a droplet radius of $3.09 \mu \mathrm{m}$. 
5. Emulsion droplet interactions
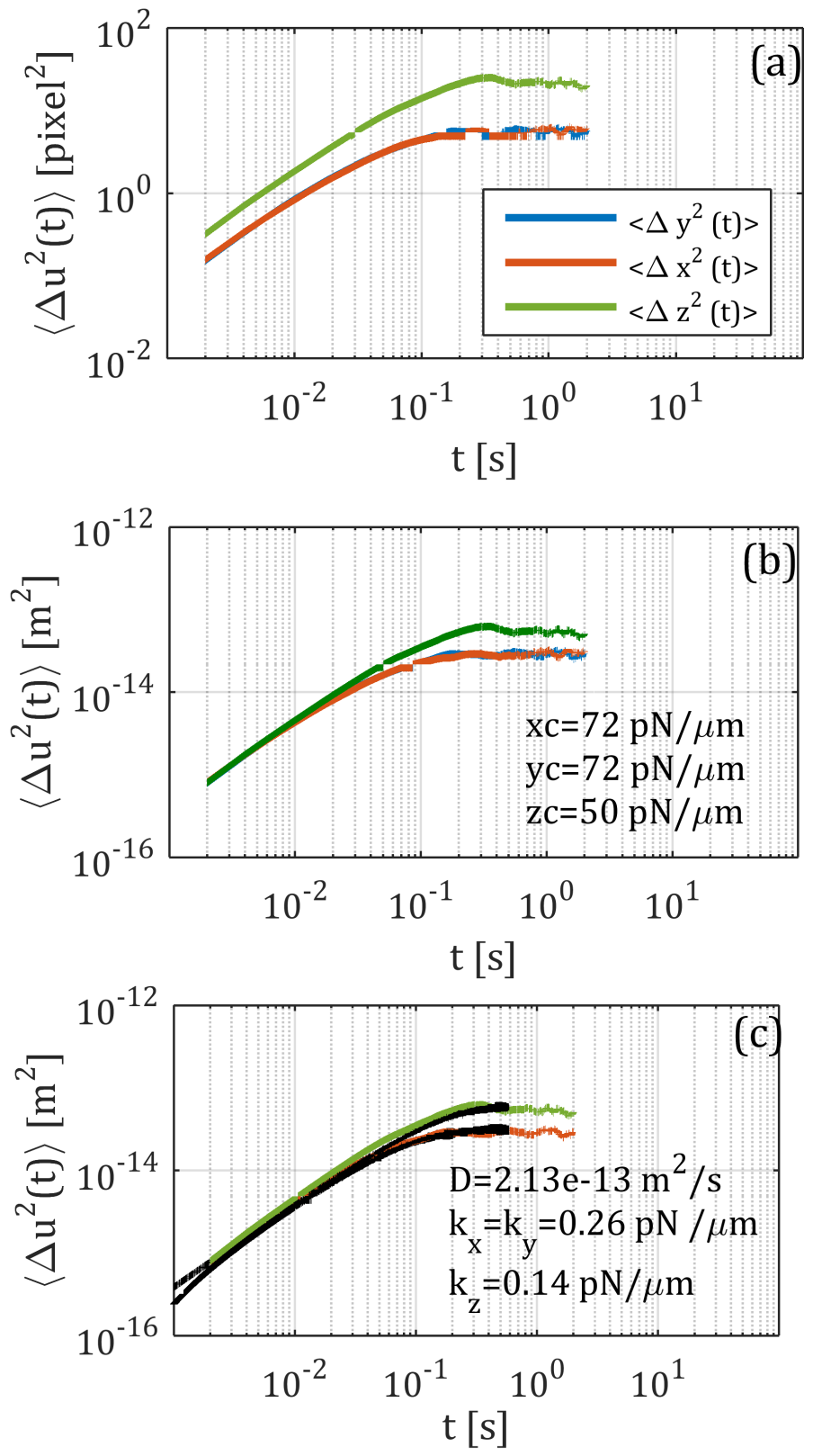

Figure 5.1.: Mean squared displacement of a $1.85 \mu \mathrm{m}$ silica bead. Plot (a) shows the uncalibrated MSD. Plot (b) shows the calibrated MSD (pixel to metre). Plot (c) shows the MSD model calculation (in black) overlaid onto the calibrated MSD. The $x$-data is in red, $y$-data in blue and $z$-data in green. 

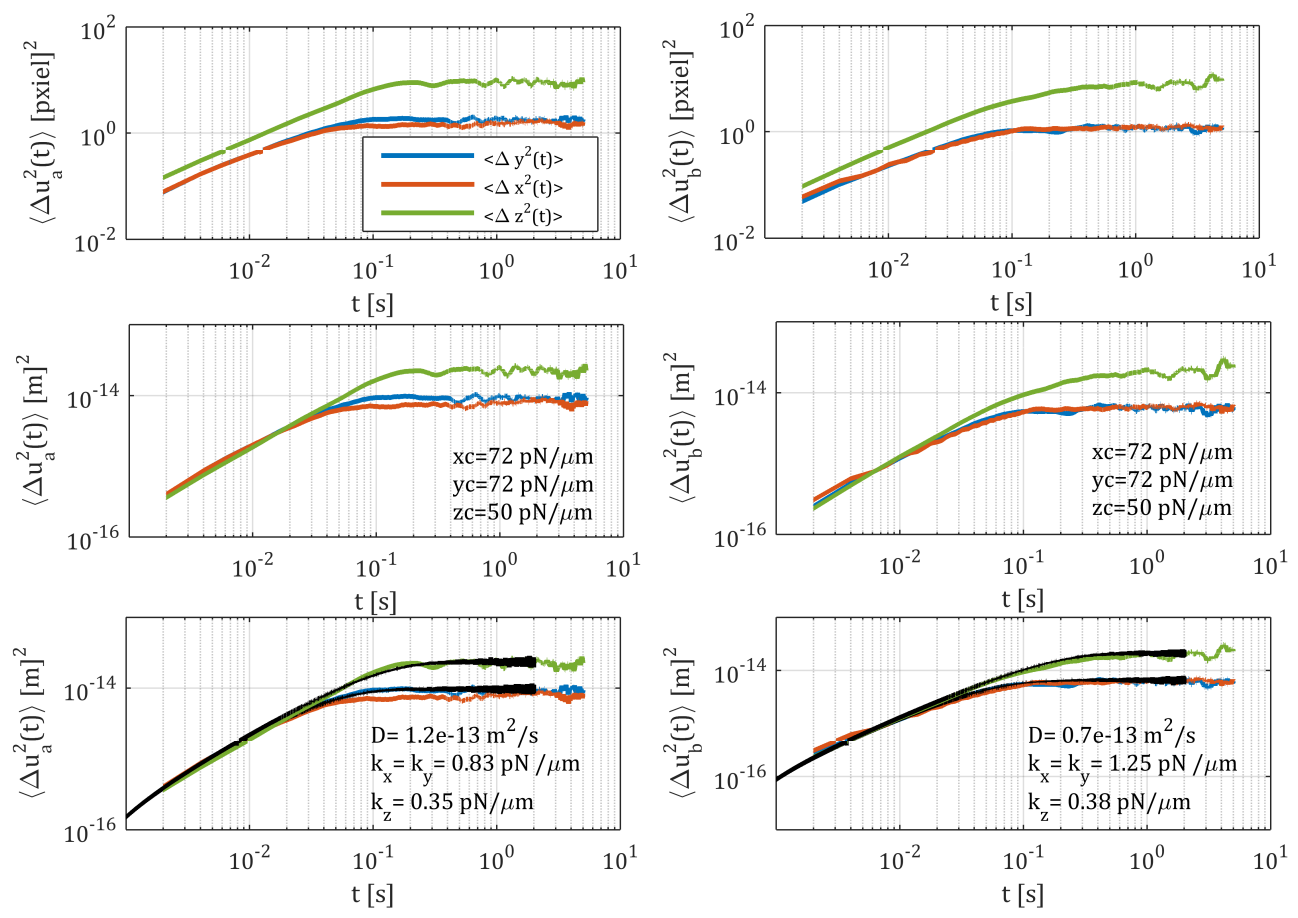

Figure 5.2.: Mean squared displacement of two different emulsion droplets (left and right columns). The first row shows the uncalibrated MSD (subplots a \& d), middle row shows the calibrated MSD (subplots $b$ \& e) and the third row shows the fits (in black) of the calibrated data (subplots $c \& \mathrm{f}$ ). The $x$-data are in red, $y$-data in blue and $z$-data in green.

Figure 5.3 shows images of the emulsion droplets which correspond to the MSD data presented in Figure 5.2 as well as the silica beads which correspond to the MSD of Figure 5.1.

\subsubsection{Droplet Interactions in Water}

First the interactions between pairs of emulsion droplets, sized as described above, were measured in water. The same method which was used to measure interactions between silica beads presented in Chapter 3 was applied to protein coated oil droplets. Figure 5.4 shows the interactions between 3 different pairs of sodium 


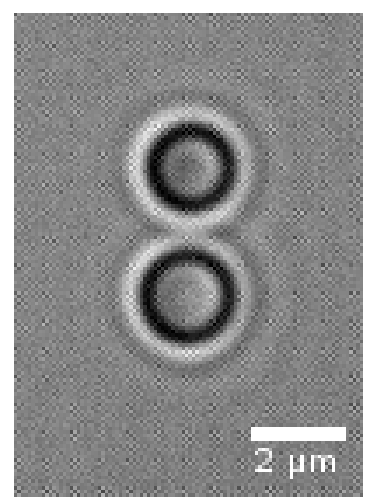

(a) Emulsion drops

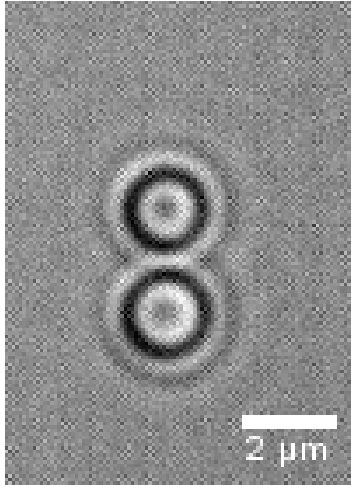

(b) Silica Beads

Figure 5.3.: Images of the emulsion droplets and silica beads.

caseinate stabilised oil droplets in water. The corresponding fits (Equation 3.5) are shown in black and found, as in the case of silica beads, to capture the data well. Images of each pair of droplets at their maximum separation are also presented in Figure 5.4.

The droplet sizes which correspond to the interaction measurements shown in Figure 5.4 are given in Table 5.1 (calculated in the same manner as discussed in Section 5.3.1). The diffusion constant for each droplet in a weak SLM trap is also presented. It can be seen that the droplets of each pair were similar in size and pair 2 had the largest droplets which agrees with our visual observation, Figure 5.4. Also, presented in Table 5.1 are the results of the fit to the electrostatic force component of the DLVO theory. It can be seen from the $R^{2}$ values that each interaction measurement had excellent agreement with the form of the model. The corresponding Debye lengths and thereby the concentrations of ionic environments (assuming a monovalent salt and room temperature) are also given. The ion concentrations are all close to that commonly reported for deionised (MilliQ) water of $\sim 10^{-5} \mathrm{mM}$ and the $\zeta$-potentials appear in reasonable agreement with the values measurement in bulk in Figure 4.18. 

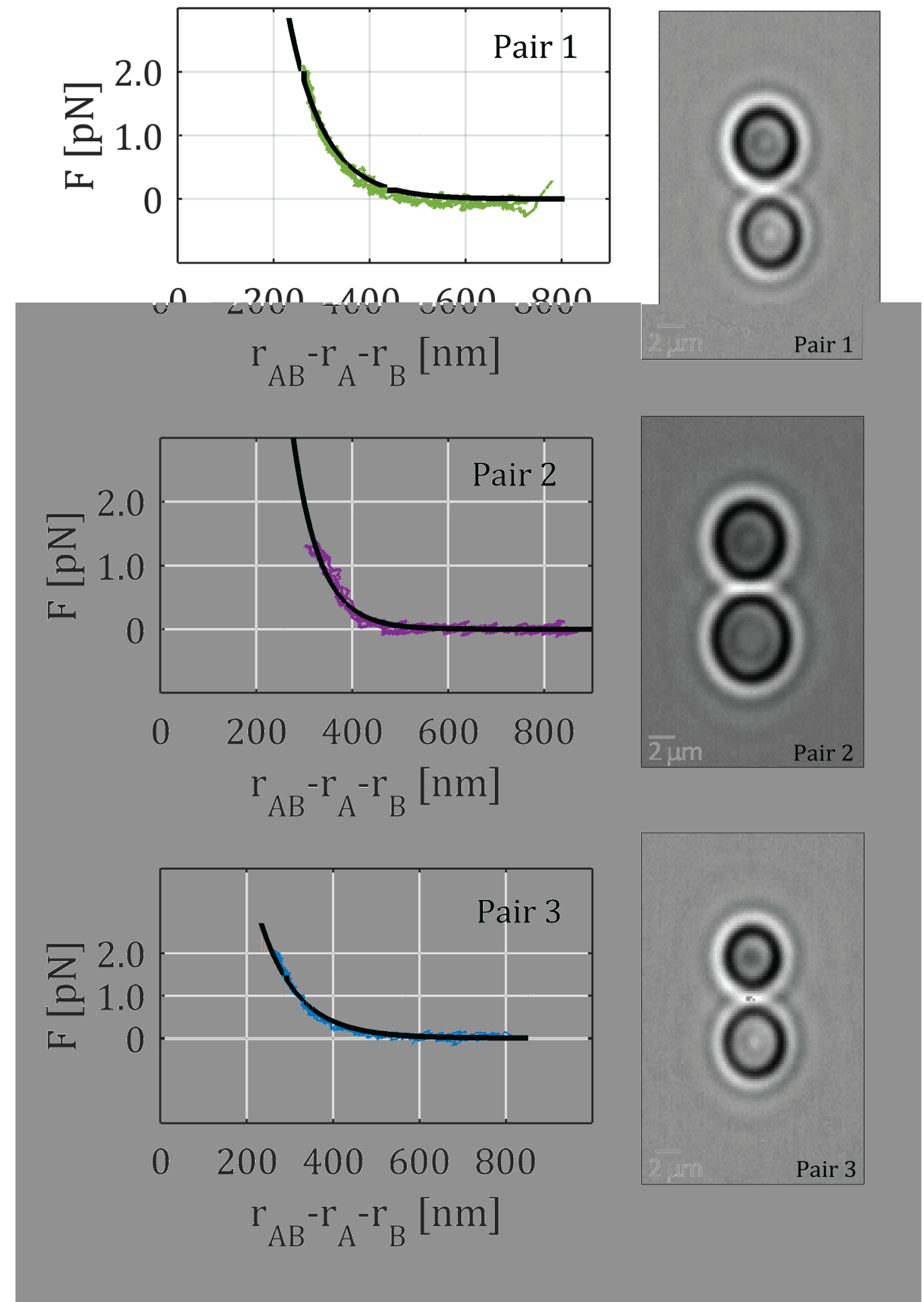

Figure 5.4.: Force displacement curves of three pairs of sodium caseinate stabilised oil droplets in water. Corresponding images of the interactions when the droplets are well-separated are shown beside their respective force curve. 


\section{Emulsion droplet interactions}

Table 5.1.: Diffusion constants $(D)$ and corresponding droplet radii $(a)$ from the fitted short time MSD behaviour for the three pairs of droplets presented in Figure 5.4. The Debye length $\left(\kappa^{-1}\right)$, salt concentration $(c)$ and charge on the droplet's surface $(Z)$ determined from the fitted force-separation curves. The $\zeta$-potential was calculated from Equation 3.6. The $R^{2}$ values show an excellent fit of the force-separation curves. Uncertainties were calculated from the 95\% confidence bounds except for the droplet radii uncertainties where an explanation is presented in Appendix $A$.

\begin{tabular}{rrrr}
\hline & \multicolumn{1}{c}{ Pair 1 } & \multicolumn{1}{c}{ Pair 2 } & \multicolumn{1}{c}{ Pair 3 } \\
\hline$D_{A}\left(\mu \mathrm{m}^{2} / \mathrm{s}\right)$ & 0.115 & 0.100 & 0.255 \\
$D_{B}\left(\mu \mathrm{m}^{2} / \mathrm{s}\right)$ & 0.115 & 0.090 & 0.225 \\
$a_{A}(\mu \mathrm{m})$ & $1.87 \pm 0.05$ & $2.15 \pm 0.05$ & $0.84 \pm 0.05$ \\
$a_{B}(\mu \mathrm{m})$ & $1.87 \pm 0.05$ & $2.38 \pm 0.05$ & $0.95 \pm 0.05$ \\
\hline$\kappa^{-} 1(\mathrm{~nm})$ & $74.5 \pm 1.3$ & $55.4 \pm 0.4$ & $87 \pm 2$ \\
$\mathrm{c}(\mu \mathrm{M})$ & $16.6 \pm 0.6$ & $30.1 \pm 0.4$ & $12.2 \pm 0.4$ \\
$\mathrm{Z}\left(\times 10^{9}\right)$ & $65 \pm 1$ & $126 \pm 10$ & $3.8 \pm 0.1$ \\
$\zeta(\mathrm{mV})$ & $-93 \pm 1$ & $-61 \pm 1$ & $-98 \pm 6$ \\
$R^{2}$ & 0.97 & 0.93 & 0.97 \\
\hline & & &
\end{tabular}

\subsubsection{Diffusion Experiment}

The nature of emulsions presents a unique challenge as droplet-to-droplet variation is expected to exist (and indeed the preliminary results reported in Table 5.1 seem to support this view in terms of $\zeta$-potential). This droplet heterogeneity may include droplet size, surface charge and distribution of charge as well as steric contributions from surfactants/emulsifiers. Therefore, to understand how a pair of droplets interact when subjected to multiple environments, the interactions must be carried out on the same pair of droplets. Here, the interactions between emulsion droplets were measured as a function of increasing salt concentration. This experiment was demonstrated on silica beads of a known size in Section 3.3.3.

Here, two emulsion droplets were trapped within a microfluidic chip at a known distance $(1.15 \mathrm{~cm})$ from a $5 \mathrm{mM}$ salt solution/water interface. The droplet size is presented in Figure 5.2, where the moving droplet has a radius of $1.80 \mu \mathrm{m}$ and the 
stationary droplet has a radius of $3.09 \mu \mathrm{m}$.

The force-displacement curves, measured over time as salt diffused from the interface, thus locally increasing the ionic strength, are presented in Figure 5.5. Here 15 interactions were collected over $200 \mathrm{~min}$. The times correspond to each interaction measurement are given in Table 5.2. The gap in time between 28 minutes and 105 minutes was the time where reference images were collected for 3D-tracking. As salt diffused into the water from the interface, the interactions between the emulsion droplets become shorter ranged resulting from shorter Debye lengths originating from the increasing ionic strength, as expected. The force curves were fitted to the electrostatic component of the DLVO theory, as described previously (Equation 3.5) and the Debye lengths, charge and corresponding salt concentrations were extracted (see Table 5.2).
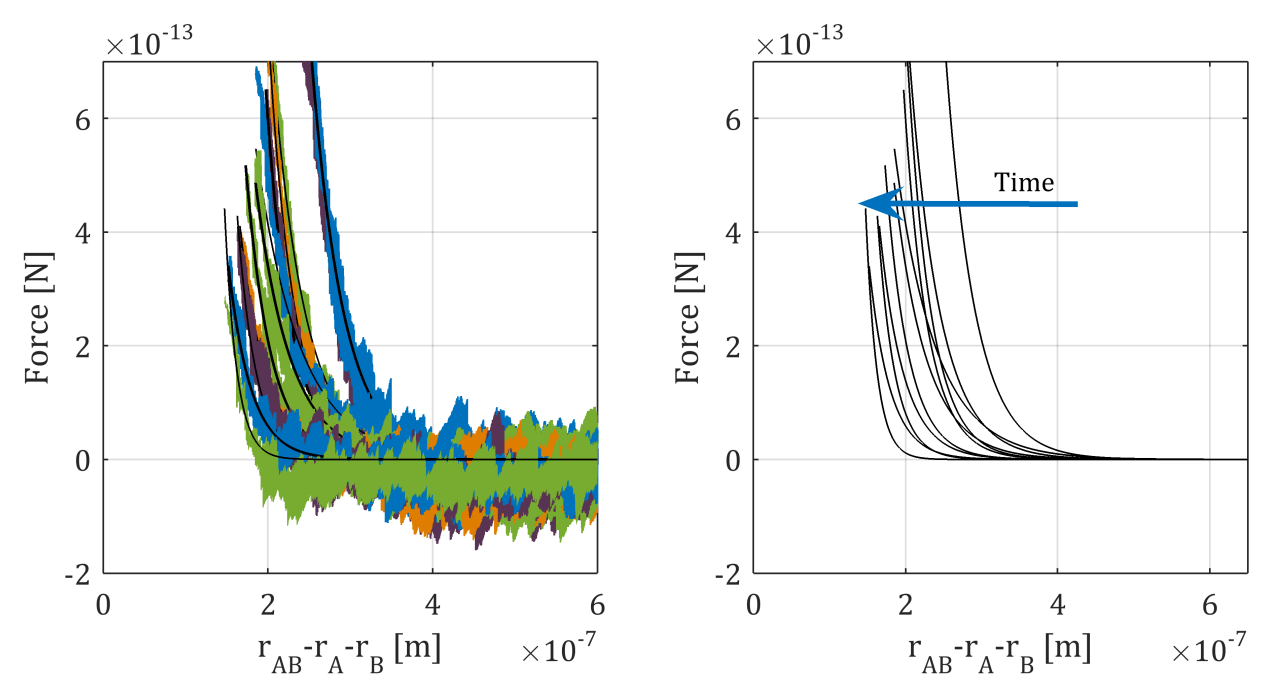

Figure 5.5.: Force displacement curves of emulsion droplets as a function of time as the local salt concentration was increasing owing to the diffusion from a $5 \mathrm{mM}$ salt solution-water interface. Fifteen measurements were taken over $200 \mathrm{~min}$. Left plot shows the interaction data in colour and their corresponding fits in solid black lines. For simplicity, the right plot shows only the fit data.

Figure 5.6 shows the salt concentration extracted from the time-resolved force curves. Here, the diffusion equation (Equation 3.10), is also presented in order to predict the salt concentration at the position of the droplets assuming that that $C_{0}=5$ 


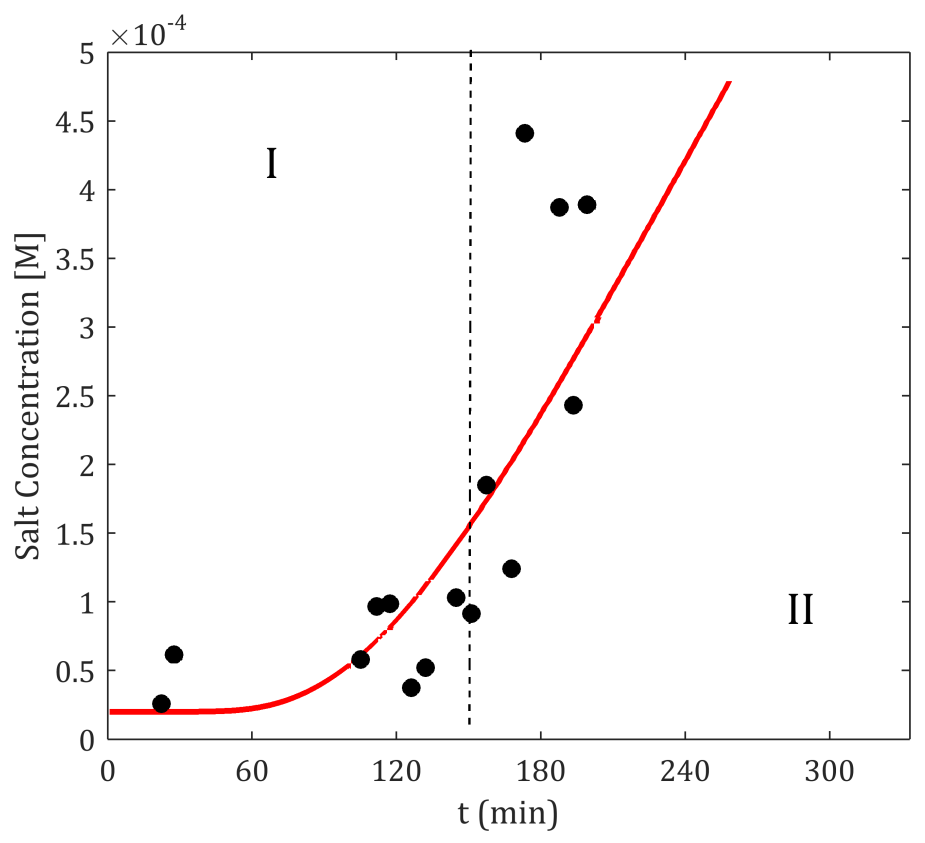

Figure 5.6.: The salt concentration extracted from the time-resolved force curves as a function of time. The diffusion formalism is shown in red. Region I, is the region where excellent exponential fits and realistic $\zeta$-potentials are found and Region II shows anomalous behaviour, where the extracted salt concentration no longer trends with the diffusion formalism. Here the root mean squared thermal fluctuations as determined by trap strength interfere - but it is noteworthy that the curves are no longer exponential signally perhaps another behaviour as the average Debye length in fact is not representative of the expected trend.

$\mathrm{mM} \mathrm{NaCl}$, and the interaction measurement takes place $1.15 \mathrm{~cm}$ from the salt/water interface. Unfortunately, due to the short distance to the salt/water interface, only 5 time-resolved force curves were measured where true-Debye lengths were recovered (See Chapter 3). The remaining 10 measurements may have dominated by the Brownian fluctuations as described in Chapter 3.

Table 5.2 presents the results of the fits to the electrostatic component of the DLVO theory. Measurements 1-5 recover length scales for the Debye length whereby realistic values should be extracted (an increase in salt concentration from $26 \mu \mathrm{M}$ to $99 \mu \mathrm{M}$ over 117 minutes). The fits all resulted in excellent agreement where the $R^{2} \geq 0.95$. Furthermore, the presented $\zeta$-potentials of the droplets were calculated 
using Equation 3.6, and reasonable values were recovered (Table 4.18). Nevertheless, these measurements show considerably more scatter than the silica bead force measurements presented in Chapter 3.

Table 5.2.: The results from fitting the force curves given in Figure 5.5 using Equation 3.5. The Debye length, corresponding salt concentration and charge on the bead are presented. Measurement numbers 1-5 result in true-force curves and measurements 6-15 is where the Debye length no longer results in strictly electrostatic behaviour.

\begin{tabular}{rrcrrrr}
\hline$\#$ & $\begin{array}{r}\text { Time } \\
(\min )\end{array}$ & $\begin{array}{c}\kappa^{-1} \\
(\mathrm{~nm})\end{array}$ & \multicolumn{1}{c}{$\begin{array}{c}\mathrm{c} \\
(\mu \mathrm{M})\end{array}$} & \multicolumn{1}{c}{$\begin{array}{c}\mathrm{Z} \\
\left(\times 10^{9}\right)\end{array}$} & $\begin{array}{c}-\zeta \\
(\mathrm{mV})\end{array}$ & $R^{2}$ \\
\hline 1 & 22 & $59.8 \pm 0.2$ & $25.8 \pm 0.2$ & $9.5 \pm 0.1$ & $84 \pm 1$ & 0.97 \\
2 & 28 & $38.8 \pm 0.2$ & $61.5 \pm 0.5$ & $28.2 \pm 0.7$ & $97 \pm 1$ & 0.97 \\
3 & 105 & $39.9 \pm 0.3$ & $58.0 \pm 0.7$ & $14.3 \pm 0.3$ & $70 \pm 2$ & 0.97 \\
4 & 112 & $30.9 \pm 0.3$ & $97 \pm 1$ & $28 \pm 1$ & $79 \pm 3$ & 0.95 \\
5 & 117 & $30.6 \pm 0.3$ & $99 \pm 2$ & $25.7 \pm 0.8$ & $74 \pm 2$ & 0.97 \\
\hline 6 & 126 & $49.6 \pm 0.2$ & $37.6 \pm 0.4$ & $6.8 \pm 0.1$ & $60 \pm 1$ & 0.95 \\
7 & 132 & $42.1 \pm 0.2$ & $52.2 \pm 0.6$ & $8.1 \pm 0.2$ & $55 \pm 2$ & 0.95 \\
8 & 145 & $29.9 \pm 0.2$ & $103 \pm 1$ & $16.7 \pm 0.5$ & $85 \pm 3$ & 0.95 \\
9 & 151 & $31.8 \pm 0.2$ & $91 \pm 1$ & $11.2 \pm 0.3$ & $50 \pm 2$ & 0.95 \\
10 & 157 & $22.4 \pm 0.2$ & $185 \pm 4$ & $32 \pm 2$ & $62 \pm 4$ & 0.89 \\
11 & 168 & $27.3 \pm 0.2$ & $124 \pm 2$ & $12.1 \pm 0.5$ & $46 \pm 2$ & 0.93 \\
12 & 173 & $14.5 \pm 0.3$ & $445 \pm 21$ & $121 \pm 30$ & $88 \pm 6$ & 0.79 \\
13 & 188 & $15.5 \pm 0.4$ & $387 \pm 18$ & $60 \pm 12$ & $62 \pm 10$ & 0.75 \\
14 & 194 & $19.5 \pm 0.5$ & $243 \pm 12$ & $18 \pm 3$ & $45 \pm 24$ & 0.74 \\
15 & 199 & $15.4 \pm 0.4$ & $389 \pm 21$ & $46 \pm 10$ & $58 \pm 12$ & 0.63 \\
\hline
\end{tabular}

\subsection{Discussion}

The interactions between pairs of protein coated emulsion droplets have been measured using the dual-trap optical tweezers method as described in Chapter 3. Here, interactions between sodium caseinate emulsions have been measured as a function of separation, for the first time. Their force-displacement curves have been fitted to 


\section{Emulsion droplet interactions}

the electrostatic component of the DLVO theory resulting in information regarding the droplet and its environment including the Debye length and droplet's surface charge. Additionally, the droplet size was determined by fitting the MSD of the droplet's restricted Brownian motion in a weak trap to a simulation where the diffusion constant is extracted.

In order to obtain a force-displacement curve the surface separation between droplets must be determined. This requires knowledge of the droplet size. The droplet size was determined by measuring the restricted diffusion of the droplet in a weak SLM trap. The diffusion constant was determined by fitting the experimental data to simulated data ${ }^{54}$ using the parameters of trap strength and the expected diffusion constant. It is worth mentioning again that care must be taken, as motional averaging or "motion blur" during long exposure time must be accounted for. This can impact the MSD result especially at short times as demonstrated by Raudsepp et al. ${ }^{54}$. The simulated data presented accounts for the exposure time $\left(t_{A}=1 \mathrm{~ms}\right)$. A limitation of this method is knowing the droplet's radius to approximately $50 \mathrm{~nm}$. This provides an error in the separation of $100 \mathrm{~nm}$. Though this "translation" has little effect on the Debye length and surface charge, the two parameters extracted from the exponential fit.

The interactions between droplets were measured first in "MilliQ-water" environments. The three pairs of droplets investigated are shown in Figure 5.4. Here, it can be seen that there was excellent agreement between the measured data and the DLVO theory. The resulting $\zeta$-potentials resulted in values of $\zeta_{1}=-93 \pm 1 \mathrm{mV}, \zeta_{2}=-61 \pm 1 \mathrm{mV}$ and $\zeta_{3}=-98 \pm 6 \mathrm{mV}$. While these values, are different than the bulk $\zeta$-potential of $\leq-50 \mathrm{mV}$ presented in Section 4.8.6. It is unknown how the ensemble average of the $\zeta$-potential in a bulk measurement relates to the charge on individual single droplets. A question that still remains is how homogeneous are these droplets? This chapter presents a methodology to address this question by repeating experiments and working up to an ensemble average. 
The discussion of droplet homogeneity was a common theme of this research. It was observed over the course of this work that droplets were not homogeneous as there were particular droplets which would floc during measurements or stick to surfaces while others did not. The idea of preferentially selecting the "good" droplets was a concern. The good droplets were those that fitted the criteria of having a radius of 1-3 $\mu \mathrm{m}$, not being a participant in a floc, and could be trapped with no interference of surfaces or surrounding droplets during measurements. This selection process, resulted in ignoring a population of droplets which behaved differently, and therefore, may have different interfacial properties and would result in different force curves. However, for demonstrating the method as applied to emulsion droplets, this lack of homogeneity was not prohibitive.

The curves extracted from the emulsion salt-diffusion experiment cannot preclude something else going at increasing ionic strength of the droplet's surroundings. One factor that could be an effect on the decreasing quality of the fits with time is that the droplet's step sequence was fixed so the droplet's closest separation from each other was the same over the course of the 200 minute measurement resulting in a smaller "nudge" or displacement of the droplet from the centre of the optical trap, owing to reduced repulsion. Consequently, the lack of the exponential fit could be attributed to the small displacement of droplet from the centre of the trap, where the motional averaging is overtaken with noise. A second factor could be associated with droplet dynamics between the interfacial layer and the local environment changing over the course of the measurement. It is well known that the ionic strength greatly impacts the protein's conformation on the interface effecting the interfacial tension, surface charge and the "flatness" or "hairiness" of the droplet's surface.

Marichal, studied sodium caseinate conformational changes as a function of $\mathrm{pH}$ and ionic strength at the air/oil interface. He found that at neutral $\mathrm{pH}$ at low ionic strength $(0.7 \mathrm{mM})$ that the casein molecules have a mild negative charge that repels them from each other, and that the hydrophilic residual groups are more immersed in the water phase ${ }^{98}$. As the ionic strength increases the solubility of the polar groups in the casein molecules reduces resulting in the migration towards the interface ${ }^{98}$. This 


\section{Emulsion droplet interactions}

conformational change on the interface could impact the droplet interactions as there are new charged groups at the interfaces at higher ionic strengths. Although, much work is required to investigate this phenomena and its effect on droplet interactions this research has shown that multiple environments can be probed with one pair of droplets allowing for such sequential studies to be carried out.

The force curves presented in this Chapter show no evidence of droplet deformation. The soft-sphere measurement in this Chapter fitted just as well as the hard-sphere measurement presented in Chapter 3, at least at low salt concentrations. The droplets or beads never actually come into contact during the interaction measurement. The long-ranged repulsion could induce a slight deformation, this is likely to be insignificant compared to the full contact force between the droplets as such in AFM or micropipette measurements ${ }^{50}$. This is an advantage for using optical tweezers for direct force measurement as the beads or droplets are free in solution and the effect of the electrical double layer on the droplet interactions is more 'real' compared to bringing droplets into contact with each other when attached to surfaces. Also, it is important to note that in studies were droplet deformation is investigated in interaction measurements the droplets are typically an order of magnitude larger than the droplets studied in this work. This results in a higher Laplace pressure in the droplets in this study as compared to larger droplets in AFM studies ${ }^{10-12,99,100}$.

Although, the optical tweezers method presented in this thesis is not fast the experimental data collection is straightforward and takes minimal sample preparation. This is advantageous over direct force measurement apparatuses such as AFM and micropipette, where droplets have to be attached to cantilevers or other surfaces, reducing the ability to quickly grab a new pair of droplets and measure many interactions. The bottleneck in these measurements is determining the positions of the beads by 3D-tracking which is out of the scope of this thesis. Though, slow, the 3D-tracking provides the ability to obtain the best resolution obtained yet for measuring interactions between colloidal particles experimentally. Finally, by holding both droplets in traps, rather than for example one with a micropipette, they are still free to rotate and there are no issues with hydrodynamics owing to the introduction 


\subsection{Conclusion}

of obstructions.

\subsection{Conclusion}

In this chapter it has been demonstrated that with the understanding gleaned from the silica particle systems, useful measurements of this type could be made on pairs of emulsion droplets. The emulsion droplets used in this study were stabilised by sodium caseinate and had a radius between 1-3 $\mu \mathrm{m}$. The droplet size was determined by the mean squared displacement of the droplet in a weak SLM trap (as demonstrated on silica beads of known size). The interactions between pairs of droplets were measured in "MilliQ" water and the data fitted well to the electrostatic force described by the DLVO theory with reasonable $\zeta$-potentials extracted. To further demonstrate this dual-trap optical tweezers method, interactions between the same pair of droplets were measured at increasing $\mathrm{NaCl}$ concentrations by means of diffusion. The expected trend has found to agree with calculations of the temporal evolution of increased local salt concentration based on a diffusion equation. However, at salt concentrations above $100 \mu \mathrm{m}$ significant deviations in the force-curves were observed that may signal salt induced changes of droplets interface or be attributed to the small magnitude of the force being within the noise. This warrants further investigation. 


\section{Concluding Remarks and Future Work}

"No one ever finishes their thesis, they abandon it."

- Pablo Etchegoin

A dual-trap optical tweezers method was developed to measure direct interactions between micrometre sized colloidal particles to a precision never achieved before. Optical tweezers utilise laser light, to manipulate particles or droplets in three dimensions. A spatial light modulator was coupled to the apparatus to control the position of a moving droplet by holography. The opposing bead or droplet was held in a stationary trap created using a fixed laser. This configuration provides a great amount of flexibility as the position of the moving particles could be controlled in 3-dimensions, while the stationary droplet was fixed.

The method developed in this research allows for quantitative information regarding the bead or droplet surface charge, and solution Debye length to be extracted. These parameters provide information on individual droplets which is difficult to understand from bulk measurements. This method provides the ability to begin to probe individual interactions and compare to bulk sample behaviour. Understanding this correlation is powerful.

Moving forward, there are several apparatus developments which would provide this method greater capability. First, improving the range of available trap strengths. Although, weak traps provide better resolution, there is a barrier to measuring 
stronger interactions at close separations. Therefore, tuning the trap strengths for different environments of the pairs of beads will be necessary.

Utilising holography in this study to position the moving bead provides a level of flexibility. However, the position of the beads was not always as accurate as we would have liked, as trap jumping was observed. Understanding, and optimising the holograms and optimising there calculations to minimise ghost traps to improve trap strengths, and provide more accurate trap positioning; especially at close separations would provide the ability to control the bead's positions to greater precision. Furthermore, this could provide the ability to utilise the SLM to control the position of both traps.

In this study, the holograms have largely restricted to those generated by Arryx were proprietary to the company. To work around this, holograms were tuned by re-loading until the beads position moved from one step to the other as expected. Implementing open source programs, such as Red Tweezers, may provide the ability for improved trap placement and greater understanding of the trap shape providing more control of the trap placement. The ability to re-use the same set of holograms is powerful and was not possible in these experiments. The holograms used in this study were generated in real time with the inability to save and reuse them.

Extensive work was carried out on developing a flow cell, where a single pair of beads or droplets could be studied under various conditions. However, this task was challenging due to weak trap strength in our apparatus, where even the slightest flow could dislodge a bead from a trap. The ability to flush environments would provide the greatest flexibility in measuring droplet interactions in new environments. The flow cells in this thesis may not need too much further work, indicated by some (inconsistent) successes during development, though for this to become reliable the available trap strengths must be increased.

There was significant computing challenges with this research as "big data" had to be stored, manoeuvred and processed. To put this into perspective, easily 1 TB of 


\section{Concluding Remarks and Future Work}

data could be collected in the course of a day's experiments. As computing becomes faster, and data storage becomes larger and less cumbersome, these direct force measurements could become routine. Additionally, concerns about motion blur, and motional averaging could be solved with larger and faster computers as more data could be stored, at reduced exposure time, reaching the limits of real-time Brownian motion.

Finally, the dual-trap optical tweezers have shown incredible potential to become a robust method to measure the interactions between either potentially deformable droplets or rigid particles. This method has some clear advantages over current methods including force, and spatial resolution is superior, sample preparation is straightforward, forces are measured in 3-dimensions, and the droplets are free in solution during measurement not wetted on surfaces. Accordingly, dual-trap optical tweezer methodology has provided the ability to measure interactions to a precision that has not yet been achieved by any other method for the study of emulsion systems, which in itself is a major achievement. This method is another tool in the toolbox of a colloid chemist, food scientist and physicists to probe interactions in soft materials. 


\section{Droplet Size Uncertainties}

To understand the uncertainty associated with the method used to determine droplet size an example of the Brownian motion simulation of a single droplet is presented at decreasing diffusion constants at a constant trap strength, and likewise at decreasing trap strength at a single diffusion constant (a reasonable approach given the largely uncorrelated relationship between them).
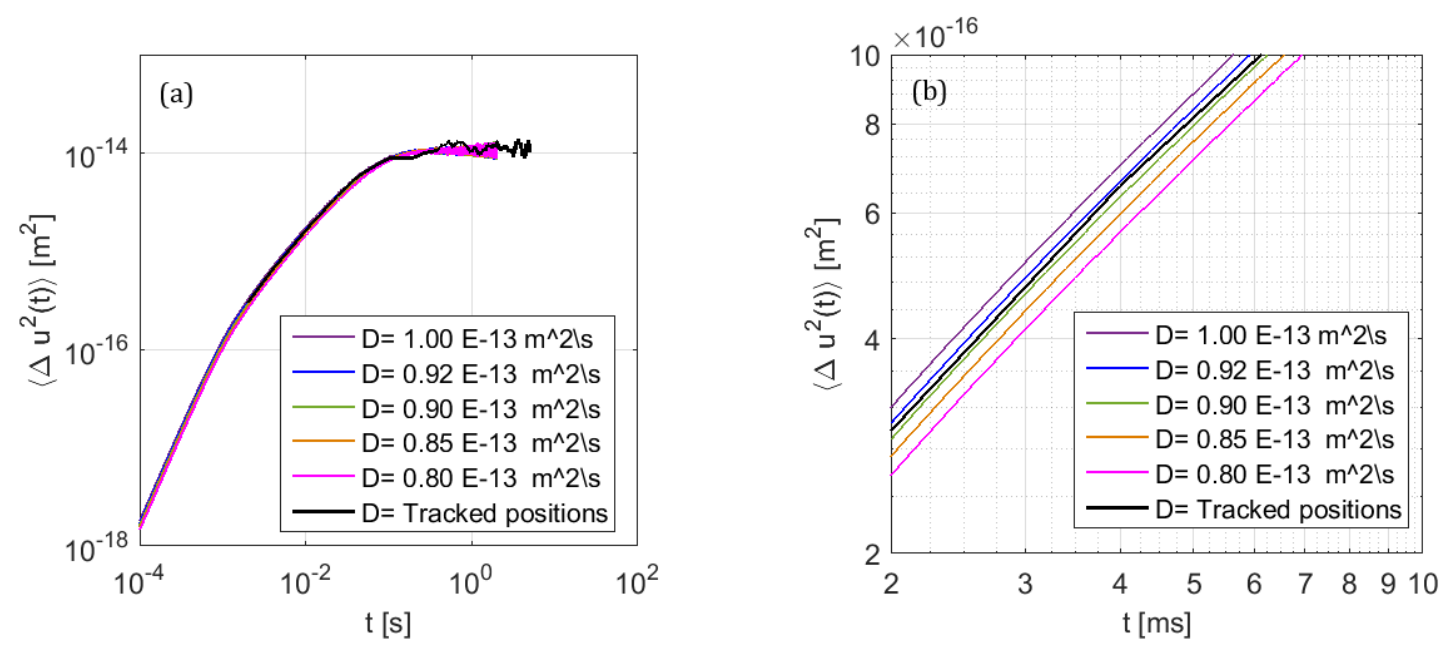

Figure A.1.: (a) Mean squared displacement of an emulsion droplet in a weak optical trap at an exposure time of $1 \mathrm{~ms}$ fitted to a Brownian motion simulation with decreasing diffusion constants where the black line shows the experimental data. (b) The initial part of the data magnified. 


\section{A. Droplet Size Uncertainties}

Due to the numerical nature of this simulation no analytical expression is available and the agreement between the calculation and the data was accessed by eye.Three variables were used to create the fit, the diffusion constant, trap strength and the exposure time. Exposure time, was constant for all MSD measurements presented in this thesis, at $1 \mathrm{~ms}$. The diffusion constant is fitted to the short time MSD behaviour which is subsequently used to extract the droplet radius, since the viscosity of the continuous phase is known. Figure A.1 shows the experimental data fitted to the Brownian simulation at diffusion constants ranging from $0.8 \mathrm{E}-13$ to $1.0 \mathrm{E}-13 \mathrm{~m} / 2 / \mathrm{s}$ where Figure A.1(a) shows that all of the presented diffusion constants seem to fit the data well.Upon further investigation of the short time behaviour, shown Figure A.1(b) the diffusion constant of $0.90 \mathrm{E}-13 \mathrm{~m}^{2} / \mathrm{s}$ fits the data "best."

To understand the possible uncertainty of the above diffusion constants, Table A.1 shows the corresponding droplet sizes which are associated with the fits. The values which have acceptable by eye fits in Figure A.1 are D=0.92 E-13 and 0.90 E-13. These values correspond to around $50 \mathrm{~nm}$ difference between the results for the droplets radius. This shows that when great care is taken with assigning diffusion constants that the resulting droplet radius maybe within $50 \mathrm{~nm}$.

\section{Table A.1.: Corresponding droplet sizes for the following diffusion constants}

\begin{tabular}{c|c}
\hline $\mathrm{D}\left(\mathrm{m}^{2} / \mathrm{s}\right)$ & $\mathrm{a}(\mu \mathrm{m})$ \\
\hline $1.0 \mathrm{E}-13$ & 2.15 \\
$0.92 \mathrm{E}-13$ & 2.33 \\
$0.90 \mathrm{E}-13$ & 2.38 \\
$0.85 \mathrm{E}-13$ & 2.52 \\
$0.8 \mathrm{E}-13$ & 2.68 \\
\hline \hline
\end{tabular}

Similarly, a comparison between the simulation and experimental data is made focusing on the effect of trap strength when the diffusion constant is maintained at $0.9 \mathrm{E}-13 \mathrm{~m}^{2} / \mathrm{s}$. Figure A.2 shows the calculations at decreasing trap strengths from $0.9-0.4 \mathrm{pN} / \mu \mathrm{m}$.Due to the noise associated with the long time MSD measurements, it is more difficult to determine precisely the trap strength that reproduces the 
measured data, but it is clear that it can be determined to better than $\pm 0.1 \mathrm{pN} / \mu \mathrm{m}$.
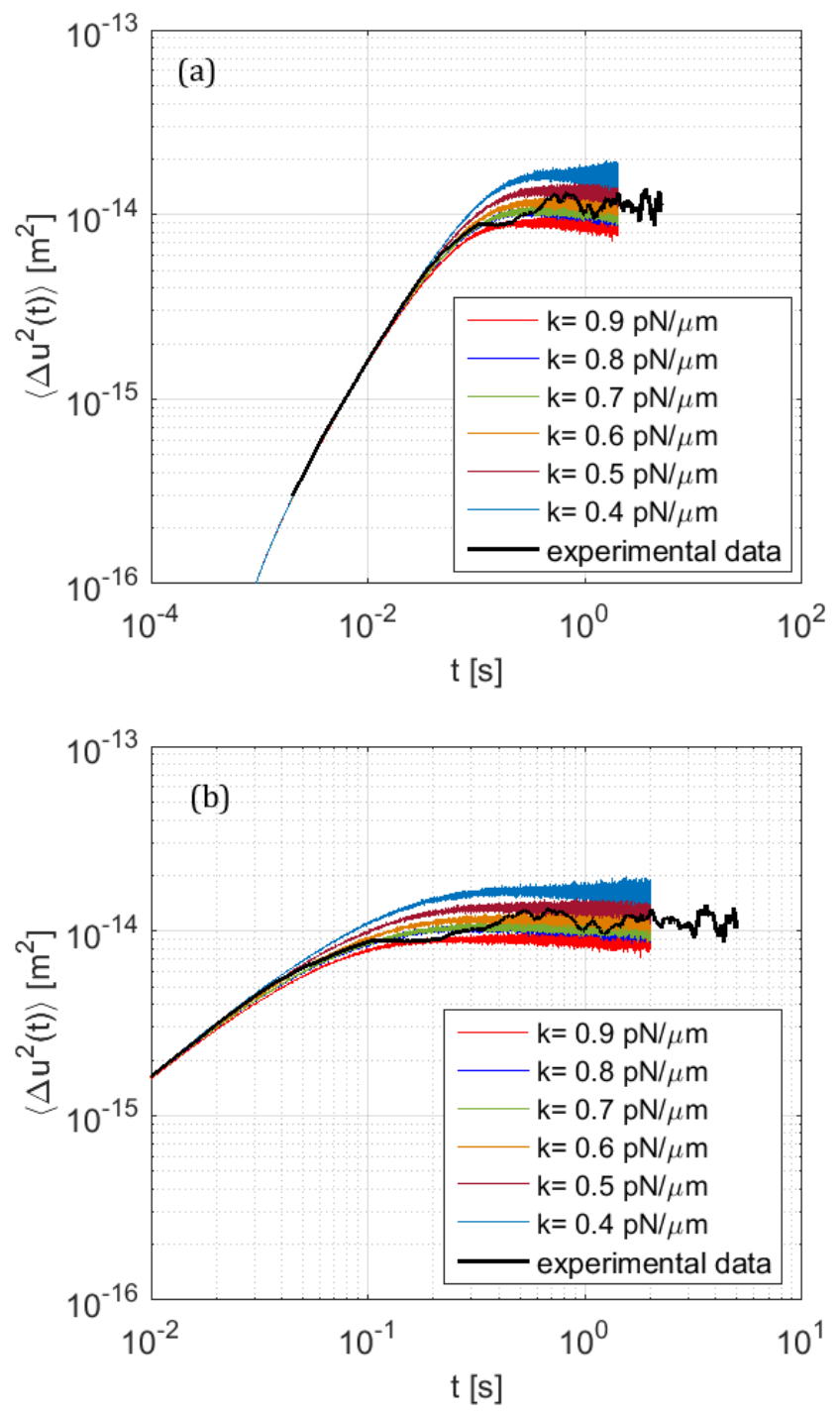

Figure A.2.: (a) MSD measurement of an emulsion droplet fitted to Brownian simulation. The fit calculations associated with decreasing trap strength are shown. Though, difficult to determine the trap strength to great accuracy, $0.7 \mathrm{pN} / \mu \mathrm{m}$ shows to be a reasonable fit to the experimental data. (b) The latter part of the data magnified. 


\section{Interaction Measurement Definitions}

1. Interaction Sequence is an image sequence where one bead is stepped toward and away from a stationary bead. The displacement of the stationary bead from the centre of the optical trap is used to determine the particle forces. The specifics of the stepping sequence was customized for each pair of beads or droplets, therefore, will be discussed alongside the data.

2. Reference image sequence was acquired for both beads of the interaction sequence as well as another image at their respective exposure time. The bead is stepped in the z-direction, in a saw-tooth pattern, 35 steps, repeating 8 times, at 0.5 pixel increments using the SLM. These images are compiled during the data tracking and is used a the kernel for the cross-correlation program.

3. Empty trap measurement is used to remove the effect of the opposing trap on the bead. An interaction sequence is repeated with one of the beads removed, measuring, as the name suggests, the empty trap approaching a bead. Data were acquired for both situations, where the empty trap is the moving and stationary traps. The sequence used for this measurement is identical to the interaction sequence.

4. $M S D$ was measured with a reduced region of interest (ROI), short exposure time, fast frame rate and a weak trap. This was used to determine the size of particles or droplets of an unknown size. 
5. Centre of Mass was required to determine the separation of the particle. The COM calculation discussed in Section 2.6.2.1, requires the beads to be well separated, this required stepping the beads away from each other and 2000 frames were collected the same data acquisition settings as the interaction sequence.

6. Background images were obtained to remove the background noise from the image. 10,000 frames are obtained with the same region of interest and microscope light intensity and averaged. 


\section{Video Tracking Method}

\section{Developing a video tracking method to study interactions between close pairs of optically trapped particles in three-dimensions}

Allan Raudsepp ${ }^{1}{ }^{*}$, Marjorie Griffiths ${ }^{2}$, Andrew J. Sutherland-Smith ${ }^{1}$, and Martin A. K. WILLIAMS ${ }^{1,2}$

${ }^{1}$ Institute of Fundamental Sciences, Massey University, New Zealand

${ }^{2}$ MacDiarmid Institute, Victoria University of Wellington, New Zealand

"Corresponding author:A.Raudsepp@massey.ac.nz

Compiled May 15, 2015

We develop a novel video tracking method that utilizes an interpolation based normalized cross correlation approach to track the position of microscopic spherical particles in three-dimensions. Subnanometer resolution is demonstrated. The method does not assume that the particle's image is radially symetric making it useful for determining position when particles are close and their images overlap. This is demonstrated in a study of the electrostatic and hydrodynamic interactions between a pair of beads in dual laser tweezers traps. (๑) 2015 Optical Society of America

OCIS codes: (100.4999) Pattern recognition, target tracking; (350.4855) Optical tweezers or optical manipulation; (180.6900) Three-dimensional microscopy.

http://dx.doi.org/10.1364/ao.XX.XXXXXX

Full text follows. 


\title{
Developing a video tracking method to study interactions between close pairs of optically trapped particles in three-dimensions
}

\author{
Allan Raudsepp ${ }^{1}, *$ Marjorie Griffiths ${ }^{2}$, Andrew J. Sutherland-Smith ${ }^{1}$, and Martin A. K. \\ WILLIAMS ${ }^{1,2}$ \\ ${ }^{1}$ Institute of Fundamental Sciences, Massey University, New Zealand \\ ${ }^{2}$ MacDiarmid Institute, Victoria University of Wellington, New Zealand \\ Corresponding author:A.Raudsepp@massey.ac.nz
}

\begin{abstract}
We develop a novel video tracking method that utilizes an interpolation based normalized cross correlation approach to track the position of microscopic spherical particles in three-dimensions. Subnanometer resolution is demonstrated. The method does not assume that the particle's image is radially symetric making it useful for determining position when particles are close and their images overlap. This is demonstrated in a study of the electrostatic and hydrodynamic interactions between a pair of beads in dual laser tweezers traps. ๑ 2015 Optical Society of America
\end{abstract}

OCIS codes: (100.4999) Pattern recognition, target tracking; (350.4855) Optical tweezers or optical manipulation; (180.6900) Three-dimensional microscopy.

\section{INTRODUCTION}

Electrostatic and hydrodynamic interactions between microscopic particles are of fundamental importance in colloidal science. Theory describing these interactions has a long history and is well developed [1][2][3]. The more recent development of laser tweezers, which can be used to trap and position colloidal particles, has begun to allow researchers to directly investigate these interactions optically [4]. With a knowledge of the characteristics of the optical traps, and size and positions of the particles, interactions between particles can be inferred. Because optical trapping is commonly conducted on an optical microscope, it combines well with video tracking, where particle position is inferred from microscopy images of the particles. Accurately determining the position of particles from bright-field or fluorescence images is of wide interest and many methods have been developed to track spherical particles in both two-dimensions (2D) and three-dimensions (3D) [5][6][7][8][9][10]. Due to interference effects, the image of a microscopic particle is typically much larger than the magnified cross section of the particle at the camera. This leads to overlap between the images of particles when particle separation is small, which is where electrostatic and hydrodynamic effects are often of most interest. Tracking methods that presuppose that particles are well separated can report misleading positions [11][12]. This has forced researchers to developed specialized tracking methods to determined po- sition when particles are close [13]. These methods are often ad-hoc and quite specific to the particle and optical set-up used; their performance under other conditions is uncertain.

To address this, we have developed a general tracking method that uses an interpolation based normalized crosscorrelation scheme to determine position of microscopic spherical particles in 3D and that can easily be adapted to exclude overlap regions in the analysis when particles are close. In this paper we will describe the method and use it to track isolated particles to characterize the methods resolution and to understand restricted diffusion in an optical trap. We will then describe the modification required for close particles and use the approach to investigate electrostatic and hydrodynamic interactions between a pair of particles trapped in a dual optical trap experiment.

\section{INTERPOLATION BASED NORMALIZED CROSS- CORRELATION}

In conventional normalized cross-correlation image analysis the position of a $2 \mathrm{D}, x_{R} \times y_{R}$, reference or template image, $R\left(x^{\prime}, y^{\prime}\right)$, is located in a larger $2 \mathrm{D}, x_{T} \times y_{T}$, target image, $T\left(x^{\prime}, y^{\prime}\right)$, by maximizing the cross-correlation, $c(x, y)$, between normalized reference and target images. Normalization ensures that the calculated cross-correlation is insensitive to changes in the average intensity. This approach can be easily extended to include a comparison between a 3D stack of aligned 2D reference images 
acquired at separate $z$ positions $R\left(x^{\prime}, y^{\prime}, z\right)$. Here the reference is located in the target by maximizing:

$$
c_{p}(x, y, z)=\frac{\sum_{x=1}^{x_{R}} \sum_{y=1}^{y_{R}} T_{p} R_{p}}{\sqrt{\sum_{x=1}^{x_{R}} \sum_{y=1}^{y_{R}} T_{p}^{2}} \sqrt{\sum_{x=1}^{x_{R}} \sum_{y=1}^{y_{R}} R_{p}^{2}}}
$$

where

$$
\begin{gathered}
T_{p}=T\left(x^{\prime}+x, y^{\prime}+y\right)-\frac{\sum_{x=1}^{x_{R}} \sum_{y=1}^{y_{R}} T\left(x^{\prime}+x, y^{\prime}+y\right)}{x_{R} y_{R}}, \\
R_{p}=R\left(x^{\prime}, y^{\prime}, z\right)-\frac{\sum_{x^{\prime}=1}^{x_{R}} \sum_{y^{\prime}=1}^{y_{R}} R\left(x^{\prime}, y^{\prime}, z\right)}{x_{R} y_{R}}
\end{gathered}
$$

with respect to the integers $x, y$ and $z$. The location of the maximum, $c_{p, \max }\left(x_{0}, y_{0}, z_{0}\right)$, determines position in $3 \mathrm{D}$ with approximately pixel level precision.

A refinement to this position can be found by maximizing:

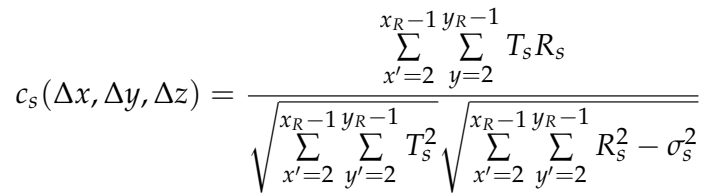

where

$$
\begin{aligned}
T_{S}= & T\left(x^{\prime}+x_{0}, y^{\prime}+y_{0}\right)-\frac{\sum_{x^{\prime}=2}^{x_{R}-1} \sum_{y^{\prime}=2}^{y_{R}-1} T\left(x^{\prime}+x_{0}, y^{\prime}+y_{0}\right)}{\left(x_{R}-2\right)\left(y_{R}-2\right)}, \\
R_{S}= & R\left(x^{\prime}-\Delta x, y^{\prime}-\Delta y, z_{0}+\Delta z\right) \\
& \quad \sum_{x^{\prime}=2}^{x_{y^{\prime}}-1} \sum_{y^{\prime}=2} R_{c}\left(x^{\prime}-\Delta x, y^{\prime}-\Delta x, z_{0}+\Delta z\right) \\
- & \frac{\left.x_{R}-2\right)\left(y_{R}-2\right)}{\sigma_{S}}=\sigma_{n}[15+\cos (2 \pi \Delta x)][15+\cos (2 \pi \Delta y)] \\
& \times[15+\cos (2 \pi \Delta z)] / 16^{3}
\end{aligned}
$$

with respect to $\Delta x, \Delta y$ and $\Delta z$. The location of this maximum, $c_{s, \max }\left(\Delta x_{0}, \Delta y_{0}, \Delta z_{0}\right)$, determines the subpixel correction required and the final position, $x=x_{0}+\Delta x_{0}, y=y_{0}+\Delta y_{0}$ and $z=z_{0}+\Delta z_{0}$ with subpixel precision. $R_{c}(x, y, z)$, in Eqn. 6 , is a three-dimensional piecewise cubic spline interpolant of $R(x, y, z)$. Once determined, this function, which passes exactly though all points in $R(x, y, z)$, can easily be evaluated for subpixel values, to fourth order accuracy. $\sigma_{S}$ is a correction for the contribution of shot noise to the variance of $R_{S}$ and includes the effect of weighted averaging that occurs during interpolation which reduces the contribution of this noise to the total variance. This correction was determined empirically by populating a three-dimensional grid with Gaussian noise of variance $\sigma_{n}^{2}$, interpolating with a piecewise cubic spline and evaluating the variance at intermediate values. It is specific to the piecewise cubic spline method used and must be reevaluated for other interpolation schemes (trilinear interpolation, for example).

\section{EXPERIMENTAL}

Particle imaging, positioning and optical trapping was conducted on an air table (Newport) mounted inverted microscope (Nikon TE2000-U) equipped with laser tweezers (Arryx Inc.), a piezo actuated translation stage (Physik Instrumente E-710-3CD) and a separate conventional manual translation stage. Particles examined were silica beads with radius $a=925 \mathrm{~nm}$ (Bangs Laboratory Inc, CV 10-15\%). Beads were imaged in deionized water filled well-slides in bright-field using a $60 \times$, numerical aperture 1.2, plan-apo water immersion objective (Nikon MRD07602) with a $1.5 \times$ auxiliary objective for a total magnification of $90 \times$. Images were acquired on a 16 bit CMOS camera (Andor Techolology Ltd. Neo DC-152Q-C00-F1) with acquisition frame rates $f_{A}=150-1026 \mathrm{~Hz}$ and exposure times $t_{A}=0.5-4.0 \mathrm{~ms}$ at a background intensity of $\langle I\rangle \simeq 20$ kcounts (unless noted otherwise). The $x \times y$ resolution of the camera at $90 \times$ magnification is $72 \times 72 \mathrm{~nm} /$ pixel. A $2 \mathrm{~W}, \lambda=1064 \mathrm{~nm}$ (Spectra-Physics) and a $5 \mathrm{~W}, \lambda=1030 \mathrm{~nm}$ laser (IPG Photonics) were used for optical trapping. The optical path of the $\lambda=1064 \mathrm{~nm}$ laser includes a reflective spatial light modulator (Boulder Nonlinear Systems) which can used control the position of this trap in 3D. The position of the optical trap produced by the $\lambda=1030 \mathrm{~nm}$ laser is fixed.

All analysis was conducted using Matlab (MathWorks). $c_{p}(x, y, z)$ was maximized using Daniel Eaton's implementation [14] of the OpenCV cvMatchTemplate routine [15] (which is considerably faster than Matlab's native implementation). $c_{S}(x, y, z)$ was calculated using the spline toolbox piecewise cubic spline routines and maximized using Matlab's default unconstrained non-linear optimization which is based on Nelder-Mead's simplex method. For a $110 \times 110$ pixel target and a $75 \times 75 \times 25$ pixel reference typical evaluation time on an Intel i7 desktop PC for $c_{p, \max }$ was $0.15 \mathrm{~s}$ and for $c_{s, \max }$ was $1.7 \mathrm{~s}$. During each maximization of $c_{s}$, the $2 \mathrm{D} 75 \times 75$ pixel matrix $R_{s}$ in Eqn. 6 is computed (from precalculated spline coefficients) 150 times, on average.

\section{RESULTS}

\section{A. One particle measurements}

Typical background corrected images of the model particle are shown in Fig. 1a at four $z$ positions. The image of the bead is seen to change significantly with $z$. Intensity line profiles through the background corrected images over the dashed line indicated in Fig. 1a are shown in Fig. 1b and make it clear that the images are not radially symmetric. Image standard deviation $\sigma=\left\langle(I-\langle I\rangle)^{2}\right\rangle^{1 / 2}$ and skewness $\gamma=\left\langle(I-\langle I\rangle)^{3}\right\rangle / \sigma^{3}$ for a series of 25 images at $\Delta z=70 \mathrm{~nm}$ increments is shown in Fig. 1c. The minimum standard deviation is defined here as $z=0$ and identified as the 'focus'. As is the suggested by the line profile, the standard deviation is largest at more extreme values of the $z$. The $z$ position of the bead can, in principle, be determined by comparing the measured statistics with the statistics of a reference [6]. While such an approach is potentially extremely fast, it can be expected to fail when the beads are close and the statistics are influenced by those of an adjacent particle. Image centroids $C_{u}=\int u|I|_{\text {thres }} d u / \int|I|_{\text {thres }} d u$ (where $u$ is $x$ or $y$ ) were computed from the background corrected images. Rectification was used to ensure that the background corrected intensity was nonnegative and thresholding was used to ensure that non-negative noise did not contribute to the centroid. Image centroids are shown in Fig. 1d. Both $C_{x}$ and $C_{y}$ vary significantly around 
the minimum of the standard deviation and skewness which could be expected to complicate localization methods based on the computation of a centroid (or based on related methods that presume radial symmetry). Localization using centroids would be further complicated by the presence of nearby particles.
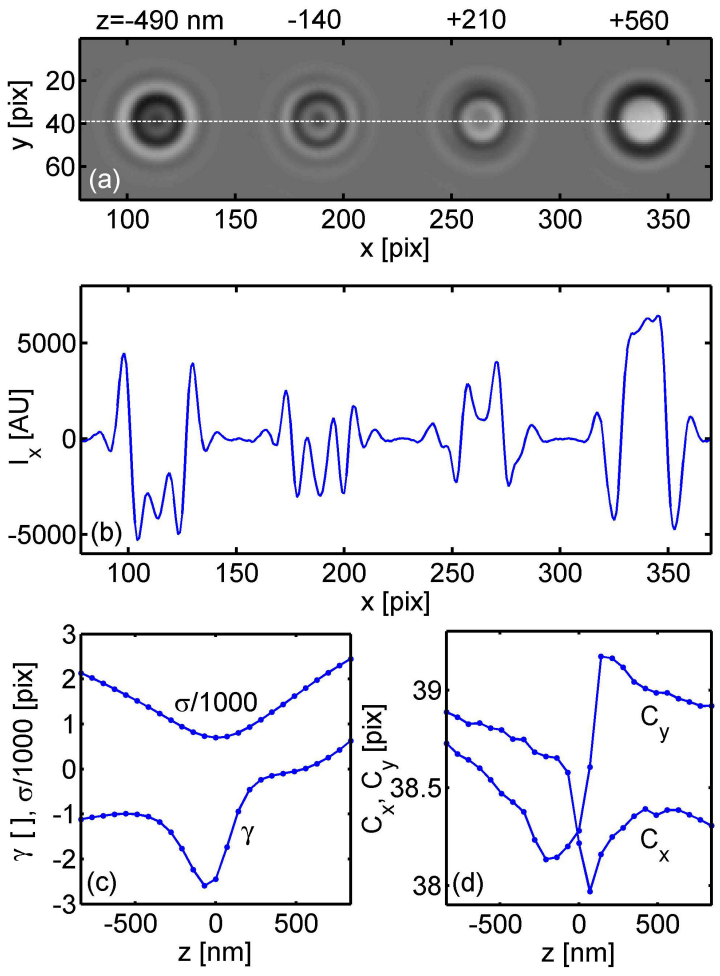

Fig. 1. Background corrected images of the silica bead showing measured intensity at selected $z$ positions (a). Associated intensity line profile (b) over the dashed line indicated in (a). Image standard deviation and skewness (c) and centroids (d) over an extended $z$ range.

The resolution of the tracking method and sensitivity to shot noise in the target images was assessed by measuring the piezostage driven translation of a cover-slip immobilized bead at two intensities. Imaging was conducted at $f_{A}=150 \mathrm{~Hz}$ and $t_{A}=4.0 \mathrm{~ms}$ and at average intensities of $\langle I\rangle \simeq 20$ kcount ( $\sigma_{n} \simeq 188$ counts) and 10 kcount ( $\sigma_{n} \simeq 135$ counts). Longer exposure times were used to suppress the effects of a small amplitude high frequency transverse oscillation present in the piezo-stage. 3000 reference images of the bead were acquired at $44, \Delta z=70 \mathrm{~nm}$ increments from $z=-910$ to $z=2100 \mathrm{~nm}$ over $20 \mathrm{~s}$. After background correction mid-step frames in the sequence were averaged over 64 frames and a $x_{R} \times y_{R} \times z_{R}, 75 \times 75 \times 44$ pixel $(5400 \times 5400 \times 3010 \mathrm{~nm})$ reference was extracted from the averaged image sequence. Averaging was used to suppress shot noise in the reference. 2000 target images were acquired as the bead was subjected to $f=1$ $\mathrm{Hz}, 10 \mathrm{~nm}$ peak-to-peak amplitude square wave displacement in both $x$ and $z$ direction over $13 \mathrm{~s}$ at $\Delta z \simeq 50 \mathrm{~nm}$ increments from $z=-900$ to $z=2100$. Target images were background corrected and the position of the bead was determined using the references and the interpolation based normalized cross-correlation method. Tracked position was dedrifted by subtracting the lowpass filtered position from the unfiltered position. Dedrifted and calibrated $x$ and $z$ positions of the bead are shown in one case
Table 1. Measured and simulated resolution

\begin{tabular}{lllll}
\hline & $\begin{array}{c}\text { mean } \\
{[\mathrm{nm}]}\end{array}$ & $\begin{array}{l}\text { min. } \\
{[\mathrm{nm}]}\end{array}$ & $\begin{array}{l}\text { max. } \\
{[\mathrm{nm}]}\end{array}$ & $\begin{array}{l}\text { std. dev. } \\
{[\mathrm{nm}]}\end{array}$ \\
\hline$\langle I\rangle=20$ kcounts & & & & \\
$\sigma_{x}$ (measured) & 0.67 & 0.53 & 0.84 & 0.071 \\
$\sigma_{z}$ (measured) & 1.2 & 0.69 & 1.8 & 0.29 \\
$\sigma_{x}$ (simulated) & 0.33 & 0.17 & 0.69 & 0.14 \\
$\sigma_{x}^{\prime}$ (simulated) & 0.67 & 0.60 & 0.89 & 0.076 \\
$\sigma_{z}$ (simulated) & 1.1 & 0.67 & 1.6 & 0.29 \\
$\langle I\rangle=10$ kcounts & & & & \\
$\sigma_{x}$ (measured) & 0.72 & 0.58 & 1.1 & 0.14 \\
$\sigma_{z}$ (measured) & 1.8 & 1.1 & 2.7 & 0.46 \\
$\sigma_{x}$ (simulated) & 0.51 & 0.28 & 1.1 & 0.19 \\
$\sigma_{x}^{\prime}$ (simulated) & 0.78 & 0.64 & 1.2 & 0.14 \\
$\sigma_{z}$ (simulated) & 1.6 & 0.98 & 2.4 & 0.43 \\
\hline
\end{tabular}

in Fig. $2 \mathrm{a}$ and 2b. Average variance at the minimum and maximum displacements of the bead was calculated at each average $z$ position, $\langle z\rangle$. The associated average standard deviation of the $x, \sigma_{x}$, and $z, \sigma_{z}$, with $\langle z\rangle$ is illustrated in Fig. $2 \mathrm{c}$ and $2 \mathrm{~d}$ for the 20 kcounts (red unfilled circle markers) and 10 kcounts (blue unfilled square markers) measurements. $\sigma_{x}<1 \mathrm{~nm}$ for the 20 kcount data over the entire range studied. With the exception of values near $\langle z\rangle=0, \sigma_{x}<1 \mathrm{~nm}$ for the 10 kcount data. $\sigma_{z}<2$ $\mathrm{nm}$ over the range examined with $\sigma_{z}<1 \mathrm{~nm}$ around $\langle z\rangle=0 \mathrm{~nm}$ for the 20 kcount data. To better understand the measured resolution, Gaussian noise, with a standard deviation matching that measured ( $\sigma_{n}=188$ counts or $\sigma_{n}=135$ counts) was added to each of the 44 averaged images that the references were extracted from, and the position of the bead in these noisy images was tracked with the references above. This was repeated 250 times for each 44 images and the standard deviation of the tracked position was evaluated; these standard deviations are also shown in Fig. $2 \mathrm{c}$ and $2 \mathrm{~d}$ for the 20 kcounts (red filled circle markers) and 10 kcounts (blue filled square markers) data. As seen in Fig. 2d, the measured and simulated $\sigma_{z}$ are very comparable indicating that the $z$ resolution is shot noise limited. The measured and simulated $\sigma_{x}$ are less comparable, with the simulated $\sigma_{x}$ smaller than the measured $\sigma_{x}$. With the inclusion of an offset in the simulated standard deviation $\sigma_{x}^{\prime}=\left[\sigma_{x}^{2}+0.57^{2}\right]^{1 / 2}$, the standard deviation calculated for the simulated 20 kcount (green upward pointing triangles) and $10 \mathrm{kcount}$ (green downward pointing triangles) data are more comparable to the measured $\sigma_{x}$, suggesting that a residual transverse stage oscillation (with a standard deviation $\sigma=0.57 \mathrm{~nm}$ ) may be limiting the measured resolution, and that much higher transverse precision is possible using the method. Measured and simulated statistics are summarized in table 1 . The methods accuracy over a larger amplitude oscillation is shown in Fig. 2e and 2f. Here, $f=0.25 \mathrm{~Hz}, 1500 \mathrm{~nm}$ peak-to-peak amplitude triangular wave displacements were applied to the bead in the $x$ and $z$ direction. Tracked positions are as expected.

The tracking shown in Fig. 2 was conducted with the cor- 

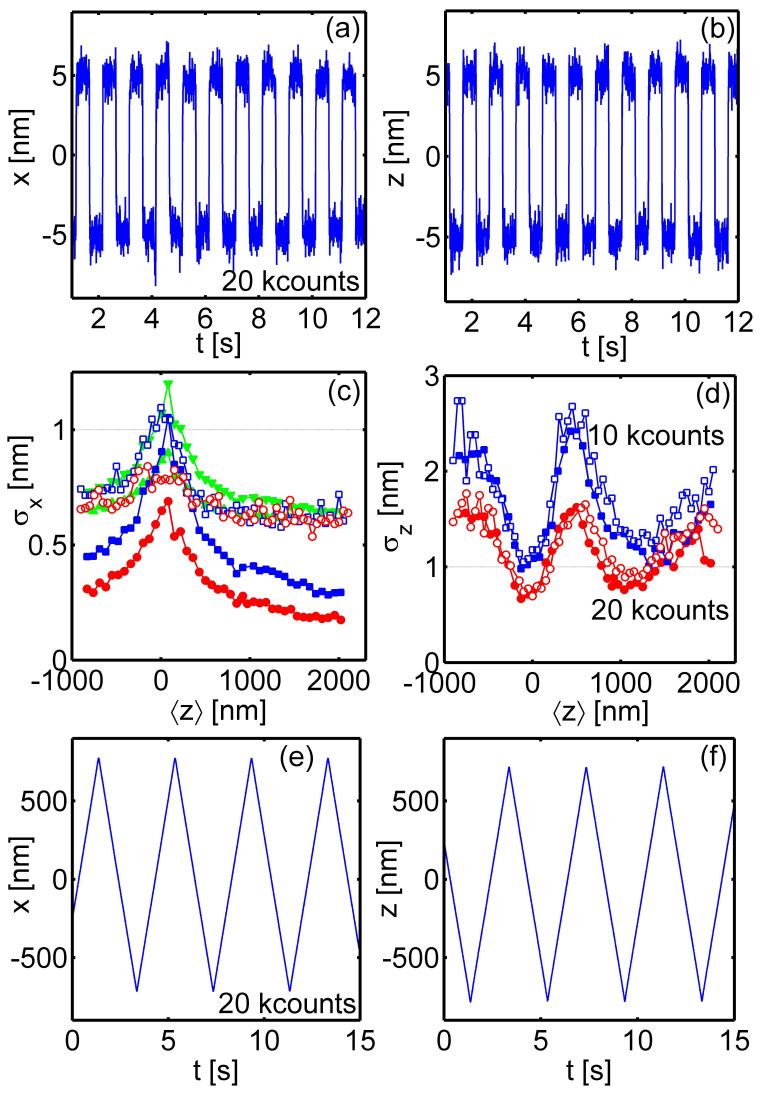

Fig. 2. Tracked $x$ (a) and $z$ (b) positions of a translated bead near the focus. Average measured (unfilled markers) and simulated (filled markers) standard deviation of tracked $x$ (c) and $z$ (d) positions with average $z$ position. Tracked $x(\mathrm{e})$ and $z$ (f) position during a large amplitude displacement.

rection term in Eqn. 6 that compensates for the contribution of shot noise to the reference. To demonstrate the importance of the correction, reference images were acquired as above and consecutive mid-step frames in the sequence were averaged over 4,16 and 64 frames. References were extracted from the mid-step average images. These references were used to track $16, f=0.16 \mathrm{~Hz}, \Delta z=1200 \mathrm{~nm}$ peak-to-peak amplitude sawtooth wave displacements of the bead. Histograms of the tracked $\bmod (z, 1)$ (modulo(dividend,divisor)) values are shown in 3 for 4, 16 and 64 frame averaged references without and with the correction. A uniform distribution is expected. Without the correction the $\bmod (z, 1)$ are biased towards mid-pixel values (Fig. 3a). Here the optimization favors the lower noise mid-pixel interpolated reference image over the higher noise on-pixel uninterpolated images. With the noise correction, which compensates for weighted averaging that occurs during interpolation, this biasing is suppressed (Fig. 3b). In lower reference noise conditions and without the correction biasing is reduced (Fig. 3c and $3 \mathrm{e}$ ) and suppressed further by the correction (Fig. $3 \mathrm{~d}$ and Fig. 3f).

It was necessary to salt the water to immobilize beads on the cover-slip. Salt screens the electrostatic repulsion between the silica beads and glass cover-slip and could be expected to influence electrostatic interaction between particles. A requirement that a particle is immobilized on a surface during acquisition of the ref-
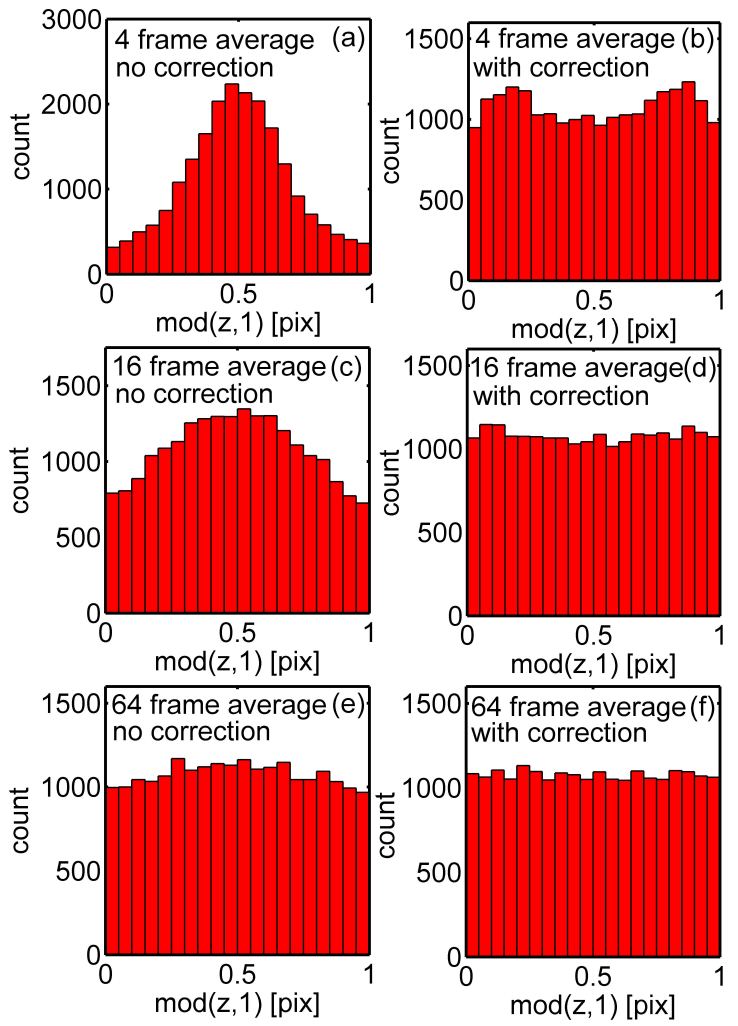

Fig. 3. Histograms of $\bmod (z, 1)$ without (a), (c) and (e), and with (b), (d) and (f) a reference noise correction, for three averaging schemes.

erence can therefore complicate experiment design. To address this complication, references were also acquired by stepping the position of the optically trapped bead in the $z$ direction by stepping the $z$ position of the trap. The trapped bead undergoes restricted diffusion in the trap and special care must be taken to minimize motional averaging that would occur when co-adding frames to suppress shot noise. These 'in-trap' references were constructed by imaging the restricted diffusion of the optically trapped particle at each step over several thousand frames, selecting a subset of the frames (typically 64) centered on the step median standard deviation and step median skewness, aligning this subset to the step average image using interpolation based normalized cross correlation and averaging the aligned subset to reduce shot noise. Fig. 4 shows the $x z$ position of a silica bead at three laser powers over $50 \mathrm{k}$ frames tracked with 'in-trap' references. As the laser power decreases the scatter in position increases. Scatter is largest in the $z$ direction which is the instrument's optical axis. Superimposed on the data are ellipses computed from the eigenvectors of the distribution using principle component analysis. These ellipses suggest that the trap is slightly angled with respect to the optical axis. 'On-surface' references were subsequently acquired for this bead. Fig. $4 \mathrm{~b}$ and $4 \mathrm{c}$ show histograms of the position of the bead acquired using the two methods. The two agree well and show that the distribution of position of the particle in the trap in both the $x$ and $z$ direction is approximately Gaussian.

The interaction between a pair of optically trapped beads depends on bead size and strength of the optical trap. These 

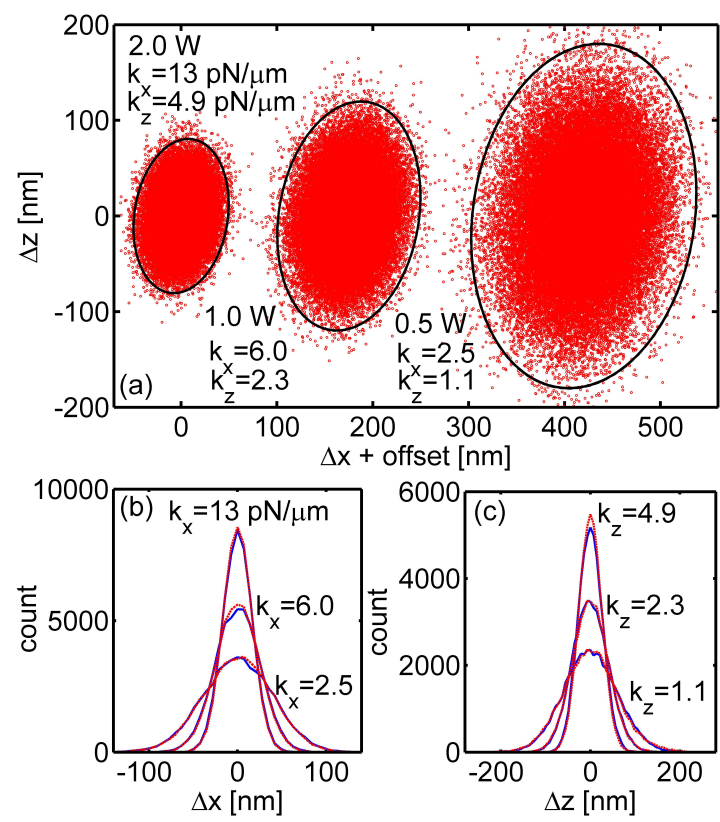

Fig. 4. Restricted diffusion of the optically trapped bead at three laser powers (a). Associated histograms computed using 'in-trap' (solid red line) and 'on-surface' (blue dashed line) references for $x(b)$ and $z$ (c).

parameters can be estimated from the mean square displacement (MSD) of the restricted diffusive motion of a bead in the trap. Because motional averaging over the exposure time can influence the measured behavior, some care is required when interpreting the MSD. This effect can be understood using Brownian dynamics simulation. For an isolated, optical trapped bead, the stochastic motion can be calculated iteratively using

$$
\begin{aligned}
u\left(t_{n+1}\right) & =u\left(t_{n}\right)+\Delta u, \\
\Delta u & =\frac{D F}{k_{B} T} \Delta t+U
\end{aligned}
$$

where $u=x, y$ or $z, D=k_{B} T /(6 \pi \eta a)$ is the diffusion constant of the bead of radius $a$ in a Newtonian medium of viscosity $\eta$ and $F$ is a restoring force due to the optical trap. For a harmonic trap, $F=-k\left[u\left(t_{n}\right)-u_{0}\right]$, where $u_{0}$ is the trap center. $U$ is a random variable chosen such that $\langle U\rangle=0$ and $\langle U U\rangle=2 D \Delta t$. The MSD associated with this stochastic motion is:

$$
\left\langle\Delta u^{2}(t)\right\rangle=2 k_{B} T / k\left(1-\exp \left[-k D t /\left(k_{B} T\right)\right]\right) .
$$

For $t \ll k_{B} T /(D k),\left\langle\Delta u^{2}(t)\right\rangle=2 D t$ and for $t \gg k_{B} T /(D k)$, $\left\langle\Delta u^{2}(t)\right\rangle=2 k_{B} T / k$ as expected [21]. Experimentally, a position, averaged over the duration of the exposure time $t_{A}$ is reported. This effect of exposure time can be included by computing a running average of the simulated position: $x_{N}\left(t_{i}\right)=(1 / N) \sum_{i=1}^{N} u\left(t_{i}\right)$ and choosing $N$ such that $t_{A}=N \Delta t$ [17]. $D$ (and $r$, if $\eta$ is known) and $k$ can be estimated by fitting the measured MSD to the simulated one. The effects of motional averaging over the exposure time on the short time MSD are demonstrated experimentally in Fig. 5 . Here $20 \mathrm{k}$ target images of an optically trapped bead were acquired with exposure times of $t_{A}=0.5,1.0 \mathrm{~ms}$ and $2.5 \mathrm{~ms}$ and frame rates of $f_{A}=1026,500$ and $250 \mathrm{~Hz} .\left\langle\Delta x^{2}(t)\right\rangle$ and $\left\langle\Delta z^{2}(t)\right\rangle$ were calculated from this data and are shown in Fig. 5. A subtle deviation of $\left\langle\Delta u^{2}(t)\right\rangle \propto t$ is observed at short times. The MSD was calculated for the three exposure times using Eqn. 8 and 9, assuming $\Delta t=0.1 \mathrm{~ms}$, $N=5,10$ or 25 , and for the trap strength constants and diffusion constant listed in figure. The simulated MSD, shown with a blue dashed line, agrees well with that measured, reducing the MSD at shorter times. If not considered, this reduction may influence the apparent diffusion constant and estimated size of the particle. The influence of the motional averaging over the exposure time on the long time/low frequency statistics and apparent trap strength constant are explored elsewhere; the model discussed here reproduces these results [17][18].

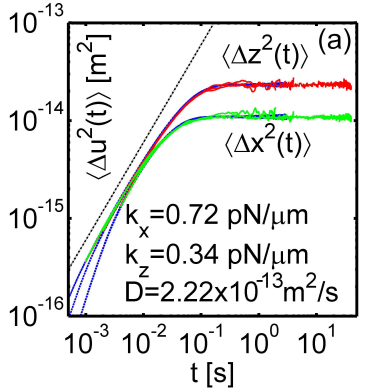

$\mathrm{t}[\mathrm{s}]$

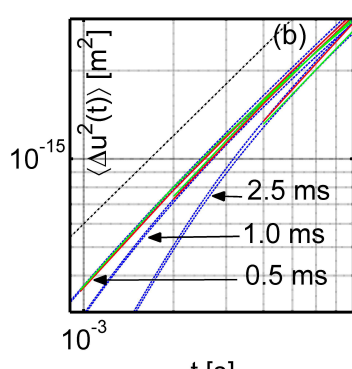

$\mathrm{t}[\mathrm{s}]$
Fig. 5. $\left\langle\Delta x^{2}(t)\right\rangle$ (green) and $\left\langle\Delta z^{2}(t)\right\rangle$ (red) measurement of the restricted diffusion of an optically trapped bead at three exposure times with model calculation (blue dashed line) (a). Expanded view of short time behavior (b). Line of slope one is included in both figures (black dashed line).

\section{B. Two particle measurements}

Electrostatic interactions between particles are typically short range. At separations required to observe these interactions the images of the particles may begin to overlap. This overlap can complicate localization of the particles. The effects of this overlap on localization can be suppressed in an approach based on cross-correlation by excluding the overlap region from the reference. This is illustrated in Fig. 6. Here, the bead labeled A was optically trapped just above the surface of the cover-slip. A second bead labeled B was immobilized on the cover slip's surface. Using the piezo-stage, a triangular $y$ displacement wave with a peak-to-peak amplitude $\Delta y_{B}=1000 \mathrm{~nm}$ and period $t=4$ $\mathrm{S}$ was applied to the cover-slip which repeatedly translates bead $B$ into bead A, nudging it slightly as it does so. Images of the bead at their maximum $\left(y_{B}=1000 \mathrm{~nm}\right)$ and minimum $\left(y_{B}=0\right.$ $\mathrm{nm})$ separations are shown in Fig. 6a. An in-trap $x_{R} \times y_{R} \times$ $z_{R}, 75 \times 75 \times 51$ pixel $(5400 \times 5400 \times 2600 \mathrm{~nm})$, reference was separately acquired for bead A. Bead A was then tracked with $75 \times 75 \times 51,75 \times 65 \times 51 \ldots 75 \times 35 \times 51$ pixel references, where region excluded from the reference was, in all cases, nearest bead B as is indicated in Fig. 6a by the white dashed lines. The average tracked $y$ position of bead A for the five references is shown in Fig. 6b. At small separation a translation, or nudge, is clearly visible in $y_{A}$ around $t=2 \mathrm{~s}$ in all cases. A translation is also observed for the $y_{R}=75,65$ and 55 pixel references at larger separations; this translation is not observed for the $y_{R}=45$ and 35 pixel references. From Eqn. 1 the maximum shot noise limited normalized cross-correlation coefficient is

$$
c_{s, \max } \simeq \sigma_{R}^{2} /\left[\left(\sigma_{R}^{2}+\sigma_{n}^{2}\right) \sigma_{R}^{2}\right]^{1 / 2}
$$


where $\sigma_{R}$ is the standard deviation of the reference, $\sigma_{n}$ is the standard deviation of shot noise in the target and it is assumed that the reference is free of shot noise. For physically reasonably values of $\sigma_{R}=1500$ (from Fig. 6c) and $\sigma_{n}=190, c_{s, \max }=0.992$. Fig. $6 \mathrm{c}$ shows the deviation of the associated maximum normalized cross-correlation coefficient from one measured during the translation. For the $y_{R}=75$ and 65 pixel references. The maximum nomalized cross-correlation coeffiecient is much lower than expected indicating that overlap is significant and suggesting that the large separation translation is an artifact. $c_{s, \max }$ for the $y_{R}=45$ and 35 pixel references are much closer to the expected value. $c_{s, \max }$ for the $y_{R}=55$ pixel reference shows a large decrease as the separation decreases indicating that overlap becomes significant when the beads are close.

To assess the effect of this cropping on repeatability the $y_{R}=75,65,55,45$ and 35 pixel references were used to track the restricted diffusion of the optically trapped bead images over $N=10 \mathrm{k}$ frames. The average deviation of the position measured from that measured using the $y_{R}=75$ pixel reference, $\sigma_{u}=\left[1 / N \sum_{i=1}^{N}\left(u_{y_{R}, i}-u_{75, i}\right)^{2}\right]^{1 / 2}$, is shown in Fig. $6 \mathrm{~d}$. The average deviation increases as the size of the reference is reduced, indicating that $y_{R}$ should be chosen to just minimize the effect of overlap on the cross-correlation to maximize repeatability.

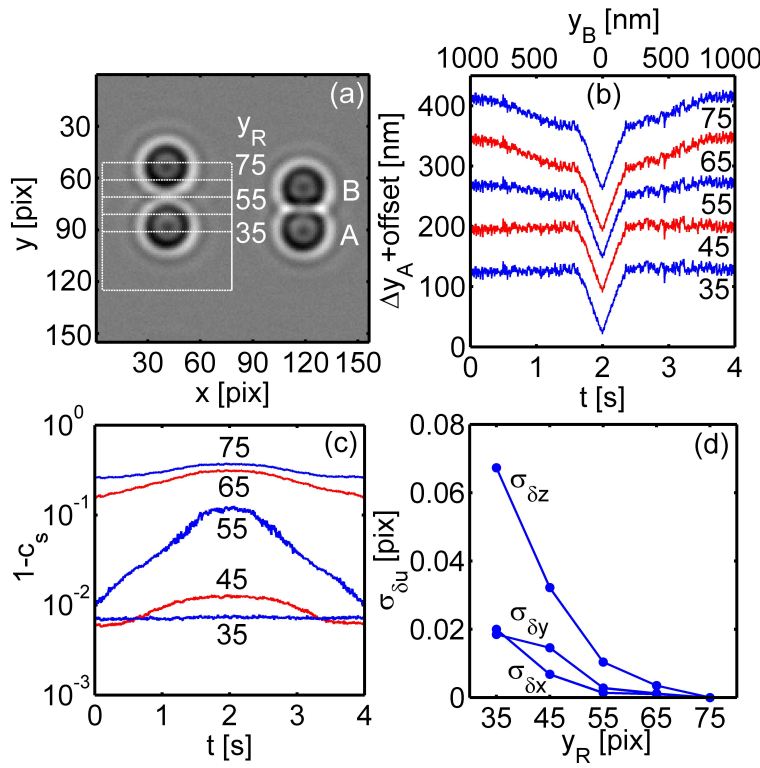

Fig. 6. Images of bead pair at their maximum and minimum separation during translation of bead B (a); $x y$ dimensions of reference are indicated with dotted line in figure. Tracked position of bead A with references with dimension $y_{R}$ indicated with time (b). Associated cross-correlation coefficient with time (c). Dependence of repeatability on $y_{R}(d)$.

With the modification to the reference required to minimize the effect of overlap understood, the interaction between a pair of close particles in matched traps can be examined. Timeaveraged and time-resolved behavior was studied. In both experiments bead $A$ is optically trapped in the fixed $\lambda=1030 \mathrm{~nm}$ trap $A$ and a bead $B$ is trapped in the movable $\lambda=1064 \mathrm{~nm}$ optical trap B.

In the time-averaged experiment a stepped triangular $y$ displacement wave with a peak-to-peak amplitude of $\Delta y \simeq 950$ $\mathrm{nm}$ (61 steps) and period of $t=120 \mathrm{~s}$ (120 steps) was applied to trap $\mathrm{B}$, at one of five $z$ positions, while the pair of beads were imaged at $f_{A}=250 \mathrm{~Hz}$ and $t_{A}=1.25 \mathrm{~ms}$ for $240 \mathrm{~s}$. Trap power for both traps was fixed at $1.0 \mathrm{~W}$. These measurements were repeated with a single bead in either trap A or B. In-trap references were acquired separately for the two beads, as well as restricted diffusion measurements which were used to size the beads and determine trap strengths. Bead sizes were estimated to be $a_{A} \simeq a_{B} \simeq 925 \mathrm{~nm}$. Trap strengths for the two traps at several trap powers are listed in table 2 . Both beads were tracked with $x_{R} \times y_{R} \times z_{R}, 75 \times 41 \times 51$ pixel references. Absolute separation was determined from the image centroid when the bead were well separated and was used to calibrate the tracked position. Calibrated, step average, $y$ and $z$ positions for one period are shown in Fig. 7. Fig. 7a to 7d shows the measured displacement each bead during the interaction measurement, Fig. $7 \mathrm{e}$ to $7 \mathrm{~h}$ shows control measurements made when the other bead is absent and Fig. $7 \mathrm{i}$ to $7 \mathrm{l}$ show the difference between the interaction and control for each of the beads. The $z$ displacement of trap B for the five position examined was approximately $z \simeq-100,0,100,200$ and $300 \mathrm{~nm}$; lines are shaded according to $z$ displacement of trap B from darkest at $z \simeq-100 \mathrm{~nm}$ to lightest at $z \simeq+300 \mathrm{~nm}$. Bead $\mathrm{A}$ is clearly nudged during the translation of bead B in most cases (Fig. 7a). An apparent large separation attraction is present. More positive $z$ displacements of trap B result in more negative displacements of bead $\mathrm{A}$ and more positive deflections of bead B during the nudge (Fig. $7 \mathrm{~b}$ and $7 \mathrm{~d}$ ). Control measurements make it clear that trap B is influencing the position of bead A (Fig. 7e and 7f) and trap A is influencing the position of bead B (Fig. 7h). The apparent large separation attraction (Fig. 7a) is less evident in the difference measurements (Fig. 7i) suggesting that the effect is due to influence of the other trap. Difference measurements also reveal that the $y$ displacement from the equilibrium position of bead B (Fig. 7k) is approximately equal and opposite to the $y$ displacement bead A (Fig. 7i). A deviation from zero in the difference measurements at larger separation is seen in some cases (most notably Fig. 7i). This was attribute to camera drift between the interaction and control measurements.

Table 2. Trap strength constants with laser power

\begin{tabular}{lllllll}
\hline power & $k_{x A}$ & $k_{y A}$ & $k_{z A}$ & $k_{x B}$ & $k_{y B}$ & $k_{z B}$ \\
{$[\mathrm{~W}]$} & {$\left[\frac{\mathrm{pN}}{\mu \mathrm{m}}\right]$} & {$\left[\frac{\mathrm{pN}}{\mu \mathrm{m}}\right]$} & {$\left[\frac{\mathrm{pN}}{\mu \mathrm{m}}\right]$} & {$\left[\frac{\mathrm{pN}}{\mu \mathrm{m}}\right]$} & {$\left[\frac{\mathrm{pN}}{\mu \mathrm{m}}\right]$} & {$\left[\frac{\mathrm{pN}}{\mu \mathrm{m}}\right]$} \\
\hline 1.0 & 5.8 & 6.0 & 1.5 & 5.3 & 6.0 & 1.8 \\
0.7 & 4.1 & 4.2 & 1.2 & 3.8 & 4.3 & 1.3 \\
0.5 & 2.9 & 3.0 & 0.80 & 2.4 & 2.8 & 0.80 \\
\hline
\end{tabular}

The electrostatic interaction force between the beads depends on their separation. Force-separation is computed in Fig. 8 for both beads. Two cases are examined. In the first case only the $y$ component of the force and separation is considered. In the second case all three components are used to compute the force-separation. Explicitly these are calculated using the dis- 

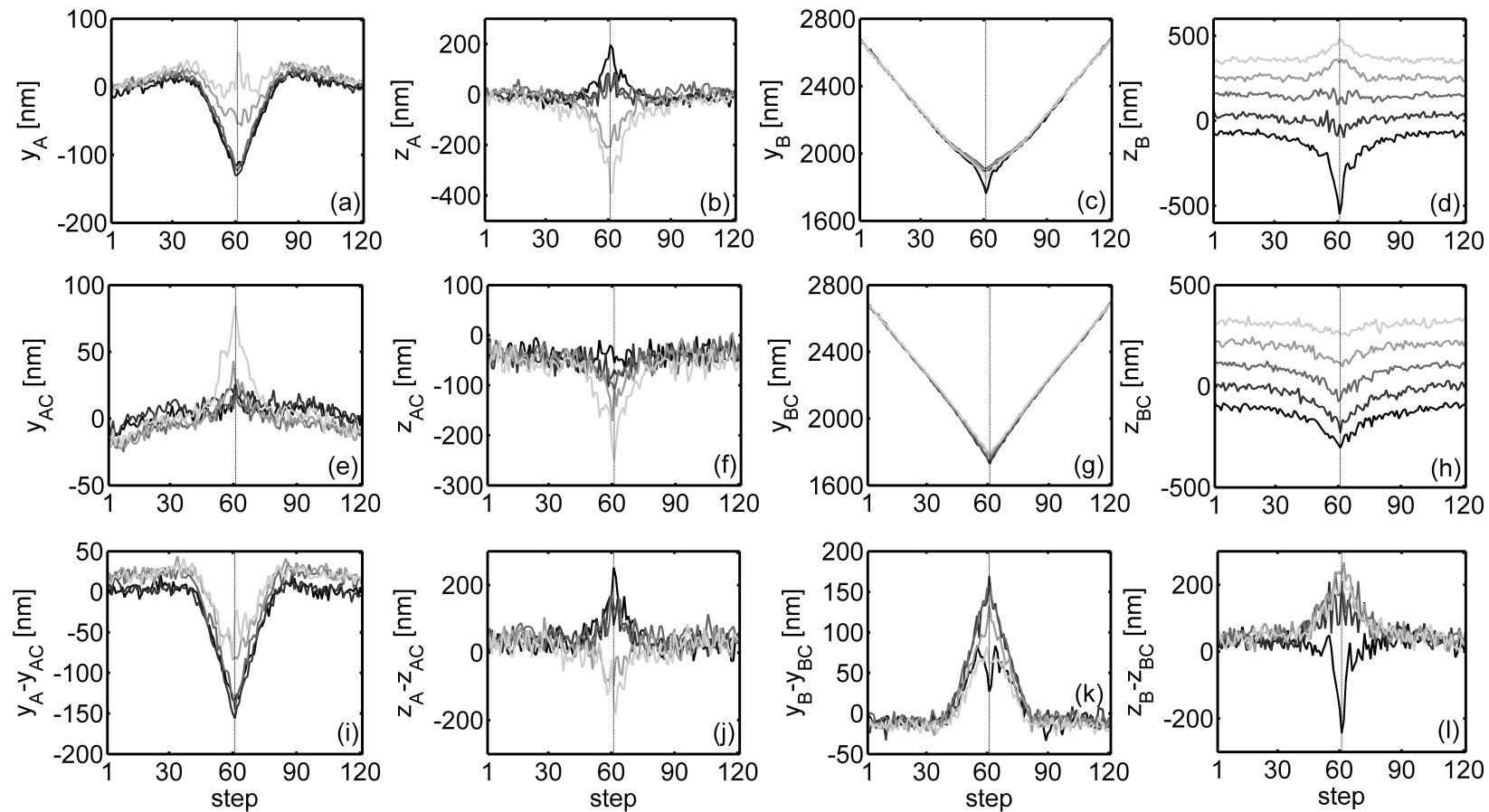

Fig. 7. $y$ and $z$ positions of bead A (a) and (b), and bead B (c) and (d) during translation of bead B. Associated control measurement for bead A (e) and (f) and bead B (g) and (h). Differences between interaction and control measurements for bead A (i) and (j), and bead $B(k)$ and $(1)$.

placements in Fig. 7, trap strengths in table 2, and the relations:

$$
\begin{aligned}
y_{A B} & =\left(y_{B}-y_{B C}\right)-\left(y_{A}-y_{A C}\right), \\
F_{y A} & =k_{y A}\left(y_{A}-y_{A C}\right), \\
F_{y B} & =k_{y B}\left(y_{B}-y_{B C}\right), \\
r_{A B} & =\left(\left[\left(x_{B}-x_{B C}\right)-\left(x_{A}-x_{A C}\right)\right]^{2}\right. \\
& +\left[\left(y_{B}-y_{B C}\right)-\left(y_{A}-y_{A C}\right)\right]^{2} \\
& \left.+\left[\left(z_{B}-z_{B C}\right)-\left(z_{A}-z_{A C}\right)\right]^{2}\right)^{1 / 2}, \\
F_{r A} & =k_{x A}\left(x_{A}-x_{A C}\right)+k_{y A}\left(y_{A}-y_{A C}\right)+k_{z A}\left(z_{A}-z_{A C}\right), \\
F_{r B} & =k_{x B}\left(x_{B}-x_{B C}\right)+k_{y B}\left(y_{B}-y_{B C}\right)+k_{z B}\left(z_{B}-z_{B C}\right) .
\end{aligned}
$$

Small offsets were included in the calculation of the difference measurements (interaction - control) to insure that the difference was zero at the maximum bead separation. Superimposed on the data is the exponential fit $F=A \exp \left[-\kappa\left(u-u_{0}\right)\right]$ where $A=1 \times 10^{-12} \mathrm{~N}, u=y_{A B}$ or $r_{A B}, u_{0}=2025 \mathrm{~nm}$ and $\kappa^{-1}=20$ $\mathrm{nm}$. Here $u$ is the center-to-center separation; in terms of the surface-to-surface separation $u^{\prime}=u-2 a, F=A^{\prime} \exp \left[-\kappa u^{\prime}\right]$ where $A^{\prime}=A \exp \left[\kappa\left(u_{0}-2 a\right)\right]=6.3 \times 10^{-9} \mathrm{~N}$, assuming $2 a=$ $1850 \mathrm{~nm}$. The three-dimensional force-separation is fitted well by the exponential model in all cases for both beads. By contrast, the one-dimensional force-separation fits well only in two cases, which occurs when deflections in the $z$ direction are small.

In the time-resolved experiment, trap B was stepped between $11 \mathrm{y}$ positions at a trap power of $0.7 \mathrm{~W}$. At each trap position the pair of beads were imaged at $f_{A}=250 \mathrm{~Hz}$ and $t_{A}=1.25 \mathrm{~ms}$ for 200 s. Additional measurements were made for selected $y$ positions at trap powers of 1.0 and $0.5 \mathrm{~W}$. The position of the particles was tracked in 3D and time-dependent normalized cross-correlations were then computed from the tracked positions according to:

$$
c_{u v}(t)=\frac{\langle(u(0)-\bar{u})(v(t)-\bar{v})\rangle}{\sigma_{u} \sigma_{v}}
$$

where $u=x_{A}, y_{A}$ or $z_{A}, v=x_{B}, y_{B}$ or $z_{B}$ and $\bar{u}$ and $\bar{v}$, and $\sigma_{u}$ and $\sigma_{v}$ are associated means and standard deviations. The crosscorrelation $c_{y A y B}(t)$ is shown in Fig. 9a for trap powers of 1.0, 0.7 and $0.5 \mathrm{~W}$ and at a center-to-center separations $r=2700 \mathrm{~nm}$. A pronounced anti-correlation is observed at a time that decreases with increasing trap power. An additional higher frequency oscillation is also observed in all correlation; this oscillation was attributed to the periodic refresh of the spatial light modulator used to generate trap B. For purely diffusive behavior it is expected that the motion of the beads would be uncorrelated and that $c_{y A y B}=0$ at all times; the presence of structure in the cross-correlation indicates that the motion of the beads are, in some way, coupled. Hydrodynamic coupling between identical beads, in identical traps (of trap strength constant $k$ ), of the component of the motion directed along the longitudinal axis leads to a cross-correlation of the form:

$$
\begin{aligned}
c_{A B}^{\|}(t)= & \frac{1}{2}\left(\exp \left[-\left(\mu_{A A}^{t t \|}+\mu_{A B}^{t t \|}\right) k|t|\right]\right. \\
& \left.-\exp \left[-\left(\mu_{A A}^{t t \|}-\mu_{A B}^{t t \|}\right) k|t|\right]\right)
\end{aligned}
$$

$\mu_{A A}^{t t \|}\left(=\mu_{B B}^{t t \|}\right)$ and $\mu_{A B}^{t t \|}$ are self- and cross- translation mobility coefficients which depend on the bead separation, bead size and viscosity [19][20][21][22]. Assuming that beads are sufficiently remote, traps are sufficiently strong and that the traps are aligned 


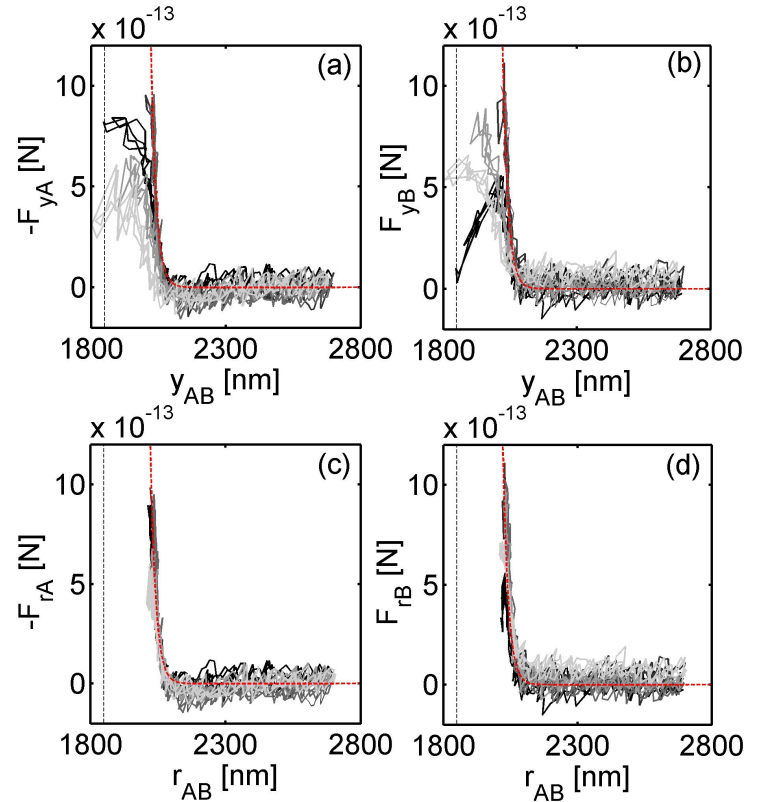

Fig. 8. Measured force-separation calculated from the $y$ position only for bead A (a) and B (b) and from the 3D position for bead A (c) and bead B (d) in gray. Exponential fit shown in all figures with a dashed red line.

in the $y$ direction, $c_{A B}^{\|}(t) \simeq c_{y A y B}(t) \cdot c_{A B}^{\|}(t)$ was computed for $\mu_{A A}^{t t \|}=0.159 /(\pi \eta a)$ and $\mu_{A B}^{t t \|}=0.081 /(\pi \eta a)$ (which are mobility coefficient corresponding to center-to-center separation $2700 \mathrm{~nm}=2.91 a$ ) and $k=6.0,4.0$ or $3.0 \mathrm{pN} / \mu \mathrm{m}$ and is shown in Fig. $9 \mathrm{~b}$. The measured cross-correlations agree with those calculated. In general, $\mu_{A A}^{t t \|} \geq \mu_{A B}^{t t \|}$ implying $c_{A B}^{\|}(t) \leq 0$ indicating that a positive cross-correlation along the longitudinal axis is not expected due to hydrodynamic coupling alone.

Measured cross-correlations for 11 y separations are shown in Fig. 9d, 9f and 9h. The associated force-separation, computed from the mean position of the bead displacement from the trap center and known trap strength is shown in Fig. 9c. Markers in Fig. 9c and lines in Fig. 9d, 9f and 9h are shaded according to the center-to-center separation of the bead with darkest lines/markers corresponding to smallest separations. A pronounced positive correlation is observed in $c_{y A y B}(t)$ at smaller separation suggesting that the electrostatic interactions observed earlier may be influencing the bead's dynamics.

Brownian dynamics simulation was used to understand the observed behavior [23]. Here the position $\mathbf{r}$ of the $i$ th bead $(i=1,2)$ was computed iteratively in time according to

$$
\begin{aligned}
\mathbf{r}_{i}\left(t_{n+1}\right) & =\mathbf{r}_{i}\left(t_{n}\right)+\Delta \mathbf{r}_{i} \\
\Delta \mathbf{r}_{i} & =\sum_{j=1,2}\left(\frac{D_{i j} \cdot \mathbf{F}_{j}}{k_{B} T}+\frac{\partial}{\partial \mathbf{r}_{j}} \cdot D_{i j}\right) \Delta t+\mathbf{R}_{i}
\end{aligned}
$$

and statistics were calculated from the simulated postitions. $D_{i j}$ is the diffusion matrix which describes diffusion in the presence of hydrodynamic coupling and depends on the self- and crosslongitudinal and transverse mobility functions: $\mu_{11}^{t t \|}, \mu_{11}^{t t \perp}, \mu_{12}^{t t \|}$ and $\mu_{12}^{t t \perp}$. These functions can be expressed in the far field as a series expansion in $a / r$, where $\mathbf{r}=\mathbf{r}_{i}-\mathbf{r}_{j}$. Explicit calculation is given elsewhere [24]; here we compute the mobility functions to $O\left((a / r)^{12}\right) . \mathbf{F}_{j}=\mathbf{F}_{j, T}+\mathbf{F}_{j, E}$ is a vector describing external forces on each of the beads which is assumed to be the sum of the trap force $\mathbf{F}_{j, T}=-k_{j} \cdot\left[\mathbf{r}_{j}\left(t_{n}\right)-\mathbf{r}_{j, 0}\right]$, where $k_{j}$ is a diagonal matrix of trap strengths and $\mathbf{r}_{j, 0}$ is the position of the center of the trap, and an electrostatic force $\mathbf{F}_{j, E}=(-1)^{j} f(r) \hat{\mathbf{r}}$. The electrostatic force is directed along the unit displacement vector $\hat{\mathbf{r}}$ with an amplitude that is dependent on center-to-center separation $r$. The prefactor $(-1)^{j}$ ensures that these forces are in opposition. $\mathbf{R}_{i}$ is a random vector chosen such that $\left\langle\mathbf{R}_{i}\right\rangle=0$ and $\left\langle\mathbf{R}_{i} \mathbf{R}_{j}\right\rangle=2 D_{i j} \Delta t$ and is calculated from the diffusion matrix using Cholesky decomposition. Positions of the beads were simulated using Eqn. 20 and 21 assuming $a_{A}=a_{B}=925 \mathrm{~nm}, \eta=1 \mathrm{mPa}$.s and trap strengths constants listed in table 2 . Positions were calculated at $\Delta t=0.05$ ms increments over $500 \mathrm{~s} \mathrm{(10} \mathrm{M} \mathrm{points)} \mathrm{at} \mathrm{each} \mathrm{trap} \mathrm{separa-}$ tion. Simulated positions were subsequently filtered using running averaging to simulate the effect of motional averaging. It was assumed that $f(r)=A_{S} \exp \left[-\kappa_{S}(r-2 a)\right]$ with $A_{S}$ and $\kappa_{S}$ chosen so that the force-separation calculated from the mean simulated position for the $1.0 \mathrm{~W}$ traps matched that measured experimentally for the $1.0 \mathrm{~W}$ traps. From these simulations it was determined that $A_{S}=500 \times 10^{-9} \mathrm{~N}$ and $\kappa_{S}^{-1}=13.2 \mathrm{~nm}$. This function is shown in Fig. 9c. With this choice of electrostatic force the force-separation was calculated from the mean simulated position in $0.7 \mathrm{~W}$ for a series of trap separations. This simulated force-separation is also shown Fig. 9c and agrees well with the measured data. Cross-correlation calculated from these simulated positions for separations closest to those measured are shown in Fig. 9e, 9g and 9i. The inclusion of the electrostatic repulsion produces a significant positive correlation at close separations.

\section{DISCUSSION}

Typical transverse and longitudinal resolution for the model $a=925 \mathrm{~nm}$ silica beads was measured as $\sigma_{x}=0.72 \mathrm{~nm}$ and $\sigma_{z}=1.2$ $\mathrm{nm}$ over $\Delta z=3000 \mathrm{~nm}$ with subnanometer resolution possible over a restricted range, in our standard illumination conditions. These resolutions are similar to experimental measurement reported elsewhere [7]. Higher sensitivities can be expected by reducing shot noise, as suggested in table 1 , or increasing the bead size [7]. These results were achieved with the inclusion of a correction for weight averaging of noise that occurs during interpolation, to suppress biasing, and indicates, more generally, that the effects of interpolation on noise should be carefully considered when fitting interpolated experimental data.

The interpolation based cross-correlation tracking approach outlined here is not fast. This is primarily because radial symmetry, which effectively reduces the dimension of the problem, is not assumed. Under the conditions described here, evaluation time is dominated by the maximization of function $c_{s}(x, y, z)$ which depends on the number of times the objective function is evaluated. Reducing the number of objective function evaluations through the use of another optimization algorithm may be possible; this was not attempted here. Evaluation time can be reduced, if necessary, by only evaluating the reference at a subset of points, or by resampling the reference and target. Resampling $x$ and $y$ by a factor of two, for example, reduces total evaluation time by factor of four. This could expected to reduce precision and accuracy, however. When $x_{R}$ and $y_{R}$ are much smaller than $x_{T}$ and $y_{T}$ the time required to maximize $c_{p}(x, y, z)$ can become considerable. Because evaluation time scales with particle number and relative size of reference to target the method outlined 
here is best suited to situations where the particle count is low and dimensions of the target and reference are well matched; these conditions are typical of laser tweezer experiments.

The transition between ballistic motion where $\left\langle\Delta u^{2}(t)\right\rangle \propto t^{2}$ and diffusive motion where $\left\langle\Delta u^{2}(t)\right\rangle \propto t$ for a particle undergoing Brownian motion is predicted to occur at $t=2 a^{2} \rho_{p} /(9 \eta)$, where $\rho_{p}$ is the density of the particle. This transition is of considerable interest but is difficult to observe because of the position sensitivity required and short times involved [25]. Motional averaging effects can be expected whenever the sampling rate (frame rate) approaches the reciprocal of the sample aperture time (exposure time). Because this averaging also produces a transition from $\left\langle\Delta u^{2}(t)\right\rangle \propto t^{2}$ to $\left\langle\Delta u^{2}(t)\right\rangle \propto t$ some care is required when attempting to observe ballistic behavior.

The time averaged behavior shown in Fig. 7 clearly indicates that bead position is influenced by the remote trap but that this effect can largely be corrected by subtracting the equilibrium position, when the bead in the remote trap is absent. These measurements illustrate the importance of control measurements in a dual trap experiment. If the bead deflection in the $z$ direction during the interaction is small, it is possible to extract the force-separation between the beads without tracking in 3D. If, however, a larger deflection is observed 3D tracking must be used.

Semi-quantitative agreement between the time-resolved measurements and Brownian dynamics demonstrates the importance of the electrostatic interaction to short range dynamic behavior. It is unclear as to why a better match between measurement and simulation was not observed here. Only trapping forces produced by the local trap were considered in the simulation; the difference may be due to the effect of the remote trap on particle motion. While positive correlations have been reported for close particles in a single harmonic potential [16], we believe that this may be the first observation in a dual trap experiment.

The time averaged force-separation measurements, like those shown in Fig. 8, are generally assumed to report directly on the electrostatic interaction between particles, with the fitted $\kappa^{-1}$ identified as the Debye length and the fitted amplitude $A^{\prime}$ related to a number of parameters including surface charge and ionic strength. Our simulations indicate that this is not necessarily the case. Here we find that $\kappa^{-1}>\kappa_{S}^{-1}$ and $A^{\prime} \ll A_{S}$. This discrepancy, which increases with decreasing trap strength appears to be associated with non-Gaussian position statistics when the beads are close and is something we are currently examining in more detail.

\section{CONCLUSION}

In this work, we developed a method for tracking microscopic spherical particles in 3D using an interpolation based normalized cross-correlation approach. The method shows similar resolution to that demonstrated by other methods. Because radial symmetry is not assumed, the method is slow, however it can be used in situations where this symmetry is absent, for example, when particles are close. This was demonstrated in a study of the static and dynamic interactions between a pair of beads in dual laser tweezers traps.

\section{ACKNOWLEDGEMENTS}

Allan Raudsepp, Bill Williams and Andrew Sutherland-Smith thank the Royal Society of New Zealand Marsden Fund for funding. Allan Raudsepp thanks Simon Hall for additional financial support during the preparation of this manuscript. Marjorie Griffiths thanks Kate McGrath for supervision.

\section{REFERENCES}

1. B. Deraguin and L. Landau, "Theory of the stability of strongly charged lyophobic sols and the adhesion of strongly charged particles in solutions of electrolytes", Acta Physiochim. URSS, 14, 633 (1941).

2. E. J. W. Verwey and J. T. G. Overbeek, "Theory of the stability of lyophobic colloids", Elsevier, Amsterdam, (1941).

3. G. K. Batchelor, "Brownian diffusion of particles with hydrodynamic interaction", J. Fluid Mech., 74, 1-29 (1976).

4. "Optical trapping and manipulation of neutral particles using lasers", Proc. Natl. Acad. Sci. USA, 94, 4853-4860 (1996)

5. J. C. Crocker and D. G. Grier, "Methods for digital video microscopy for colloidal studies", J. Colloid Interface Sci., 179, 298-310 (1996).

6. Poul Martin Hansen, Jakob Kisbye Dreyer, Jesper Ferkinghoff-Borg and Lene Oddershede, "Novel optical and statistical methods reveal colloidwall interactions inconsistent with DLVO and Lifshiftz theories", J. Colloid Interface Sci., 287, 561-571 (2005)

7. Zhipeng Zhang and Chia-Hsiang Menq, "Three-Dimensional particle tracking with subnanometer resolution using off-focus images", Appl. Opt., 47(13), 2361-2370 (2008).

8. Zhipeng Zhang and Chia-Hsiang Menq, "Best linear unbiased axial localization in three dimensional fluorescent bead tracking with subnanometer resolution using off-focus images ", JOSA A, 26(6), 1484-1493 (2009).

9. Yongxiang Gao and Maria L. Kilfoil, "Accurate detection and complete tracking of large populations of features in three dimensions", Opt. Express, 17(6), 4685-4704 (2009).

10. Marijn T. J. Loenhout, Jocab W. J. Kerssemakers, Iwijn De Vlaminck and Cess Dekker, "Non-biased-limited tracking of spherical particles, enabling nanometer resolution at low magnification", Biophys. J., 102, 2363-2371 (2012).

11. Jörg Baumgartl and Clemens Bechinger, "On the limits of digital microscopy", Europhys. Lett., 71(3), 487-493 (2005).

12. Jörg Baumgartl, Jose Luis Arauz-Lara and Clemens Bechinger, "Likecharge attraction in confinement: myth of truth", Soft Matter, 2, 631-635 (2006).

13. C. Gutsche, U. F. Keyser, K. Kegler and F. Kremer, "Forces between single pairs of charged colloids in aqueous salt solutions", PRE, 76, 031403 (2007).

14. www.cs.ubc.ca/research/deaton/remarks ncc.html

15. docs.opencv.org/modules/imgproc/doc/object_detection.html

16. Benjamin Tränkel, Micheal Speide and Alexander Rohrbach, "Interaction dynamics of two colloids in a single optical trap", PRE, 86, 021401021406 (2012).

17. Wesley P. Wong and Ken Halvorsen, "The effect of integration time on fluctuation measurements: calibrating an optical trap in the presence of motion blur", Opt. Express, 14(25), 12517-12531 (2006).

18. Astrid van der Horst and Nancy R. Forde, "Power spectral analysis for optical trap stiffness calibration from high speed camera position detection with limited bandwidth", Opt. Express, 18(8), 7670-7677 (2010).

19. Jens-Christian Meiners and Stephen R. Quake, "Direct measurement of hydrodynamic cross correlations between two particles in an external potential”, Phys. Rev. Lett., 82(10), 2211-2214 (1999).

20. P. Bartlett and S. I. Henderson and S. J. Mitchell, "Measurement of the hydrodynamic forces between two polymer-coated sphere", Philos. Trans. R. Soc. London Ser. A, 359, 883 (2001).

21. Stuart Henderson, Steven Mitchell and Paul Bartlett, "Direct measurement of colloidal friction coefficients", PRE, 64, 061403 (2001).

22. Micheal Reichert, "Hydrodynamics interactions in colloidal and biological systems", Konstanzer Online-Publikations-System (2006), http://www.ub.uni-konstanz.de/kops/volltexte/2006/1930/

23. Donald L. Ermak and J. A. McCammon, "Brownian dynamics with hydrodynamic interactions", J. Chem. Phys, 69(4), 1352-1360 (2011).

24. S. Kim and S. Karrilla, "Microhydrodynamics: Principles and selected applications", Butterworth-Heinemann, Stoneham MA (1991).

25. Rongxin Huang, Issac Chavez, Katja M. Taute, Branimir Lukic, Sylvia Jeney, Mark G. Raizen and Ernt-Ludwig Florin, "Direct observation of 
the full transition from ballistic to diffusive Brownian motion in a liquid", Nature Phys., 7, 576-580 (2011).
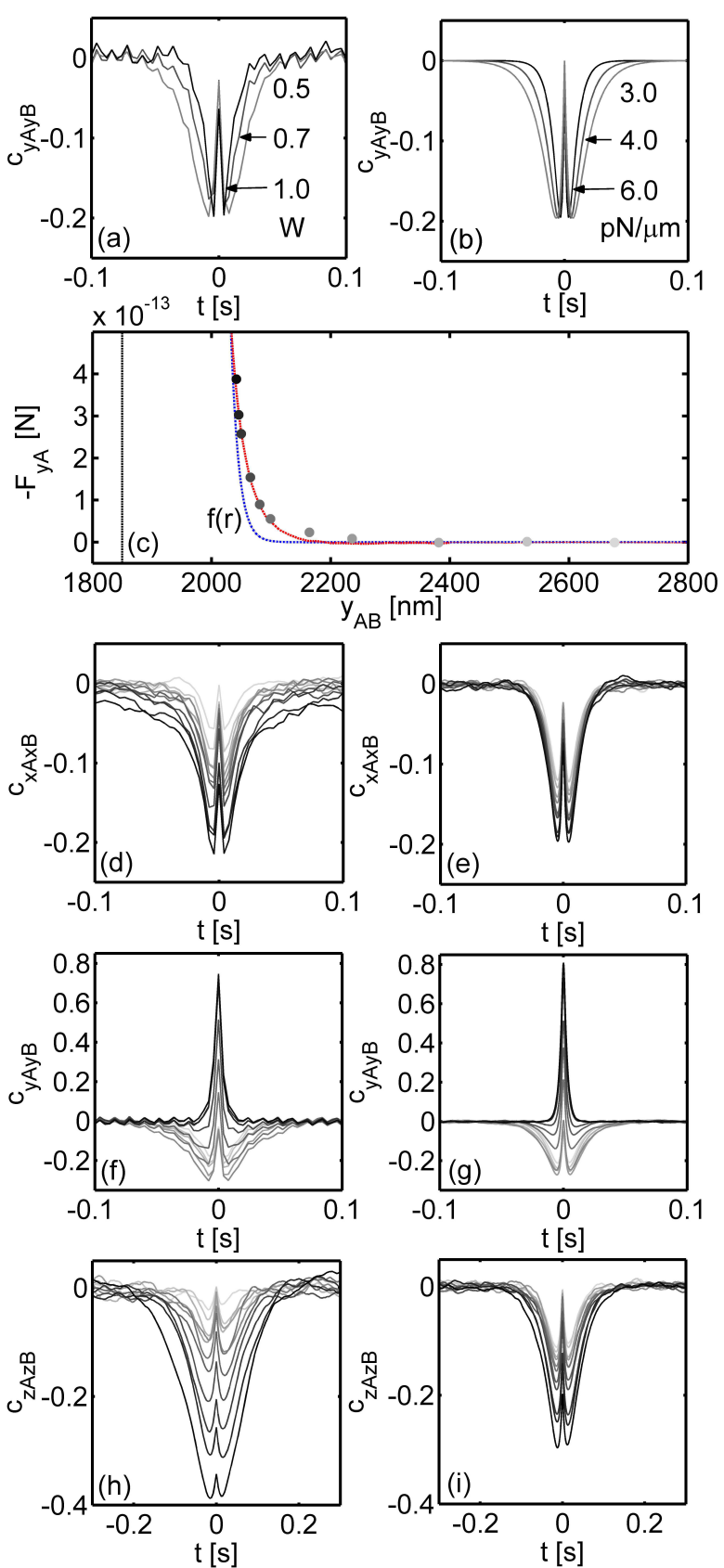

Fig. 9. Measured cross-correlation $c_{y A y B}(t)$ at three laser powers when beads are separated by approximately one radius (a). Analytic calculation of cross-correlation (b). Measured force-separation (gray markers) with calculated forceseparation (red) dashed lines for the electrostatic repulsion (blue) dot-dashed line (c). Measured cross-correlation $c_{x A x B}(t)$ (d), $c_{y A y B}(t)(\mathrm{f})$ and $c_{z A z B}(t)(\mathrm{h})$. Simulated cross-correlations $c_{x A x B}(t)(\mathrm{e}), c_{y A y B}(t)(\mathrm{g})$ and $c_{z A z B}(t)(\mathrm{i})$. 


\section{References}

(1) Craig, V. S. (1997). An historical review of surface force measurement techniques. Colloids and Surfaces A: Physicochemical and Engineering Aspects 129, 75-93.

(2) Liang, Y., Hilal, N., Langston, P., and Starov, V. (2007). Interaction forces between colloidal particles in liquid: Theory and experiment. Advances in Colloid and Interface Science 134, 151-166.

(3) Overbeek, J., and Sparnaay, M. (1952). Experiments on long-range attractive forces between macroscopic objects. Journal of Colloid Science 7, 343 -345.

(4) Derjaguin, B., Titijevskaia, A., Abricossova, I., and Malkina, A. (1954). Investigations of the forces of interaction of surfaces in different media and their application to the problem of colloid stability. Discussions of the Faraday Society 18, 24-41.

(5) Derjaguin, B., and Abrikossova, I. (1958). Direct measurements of molecular attraction of solids. Journal of Physics and Chemistry of Solids 5, 1-10.

(6) Tabor, D. (1969). Recent studies of short range forces. Journal of Colloid and Interface Science 31, $364-371$.

(7) Israelachvili, J. N., and Adams, G. E. (1978). Measurement of forces between two mica surfaces in aqueous electrolyte solutions in the range 0-100 $\mathrm{nm}$. Journal of the Chemical Society, Faraday Transactions 1: Physical Chemistry in Condensed Phases 74, 975-1001. 


\section{References}

(8) Villey, R., Piednoir, A., Sharma, P., Cottin-Bizonne, C., Cross, B., PhanerGoutorbe, M., and Charlaix, É. (2013). Capacitive detection of buried interfaces by a dynamic surface force apparatus. Review of Scientific Instruments 84, 085113.

(9) Binnig, G., Quate, C. F., and Gerber, C. (1986). Atomic force microscope. Physical Review Letters 56, 930.

(10) Tabor, R. F., Grieser, F., Dagastine, R. R., and Chan, D. Y. C. (2012). Measurement and analysis of forces in bubble and droplet systems using AFM. Journal of Colloid and Interface Science 371, 1-14.

(11) Gunning, A. P., Mackie, A. R., Wilde, P. J., and Morris, V. J. (2004). Atomic Force Microscopy of Emulsion Droplets: Probing DropletDroplet Interactions. Langmuir 20, 116-122.

(12) Dagastine, R. R., Stevens, G. W., Chan, D., and Grieser, F. (2004). Forces between two oil drops in aqueous solution measured by AFM. Journal of Colloid and Interface Science 273, 339-342.

(13) Evans, E., and Needham, D. (1987). Physical properties of surfactant bilayer membranes: thermal transitions, elasticity, rigidity, cohesion and colloidal interactions. Journal of Physical Chemistry 91, 4219-28.

(14) Yeung, A., Dabros, T., and Masliyah, J. (1998). Does Equilibrium Interfacial Tension Depend on Method of Measurement? Journal of Colloid and Interface Science 208, $241-247$.

(15) Moran, K., Yeung, A., and Masliyah, J. (1999). Measuring Interfacial Tensions of Micrometer-Sized Droplets: A Novel Micromechanical Technique. Langmuir $15,8497-8504$.

(16) Frostad, J. M., Collins, M. C., and Leal, L. G. (2013). Cantilevered-Capillary Force Apparatus for Measuring Multiphase Fluid Interactions. Langmuir 29, $4715-4725$. 
(17) Feng, X., Mussone, P., Gao, S., Wang, S., Wu, S.-Y., Masliyah, J. H., and $\mathrm{Xu}$, Z. (2009). Mechanistic Study on Demulsification of Water-in-Diluted Bitumen Emulsions by Ethylcellulose., English Langmuir 26, 3050-3057.

(18) Frostad, J. M., Collins, M. C., and Leal, L. G. (2014). Direct measurement of the interaction of model food emulsion droplets adhering by arrested coalescence. Colloids and Surfaces A: Physicochemical and Engineering Aspects $441,459-465$.

(19) Simmons, R., Finer, J., Chu, S., and Spudich, J. (1996). Quantitative measurements of force and displacement using an optical trap. Biophysical Journal 70, 1813-1822.

(20) Calderon, F. L., Stora, T., Mondain Monval, O., Poulin, P., and Bibette, J. (1994). Direct measurement of colloidal forces. Phys. Rev. Lett. 72, 2959-2962.

(21) Dreyfus, R, Lacoste, D, Bibette, J, and Baudry, J (2009). Measuring colloidal forces with the magnetic chaining technique. The European physical journal. E, Soft matter 28, 113-23.

(22) Dimitrova, T. D., Leal-Calderon, F., Gurkov, T. D., and Campbell, B. (2004). Surface forces in model oil-in-water emulsions stabilized by proteins. Advances in colloid and interface science 108-109, 73-86.

(23) Philip, J., Prakash, G. G., Jaykumar, T., Kalyanasundaram, P., MondainMonval, O., and Raj, B. (2002). Interaction between Emulsion Droplets in the Presence of PolymerSurfactant Complexes. Langmuir 18, 4625-4631.

(24) Bowman, R. W., and Padgett, M. J. (2013). Optical trapping and binding. Reports on progress in physics. Physical Society (Great Britain) 76, 026401.

(25) Ashkin, A. (1970). Acceleration and trapping of particles by radiation pressure. Physical review letters 24, 156-158.

(26) Ashkin, A. (1970). Atomic-Beam Deflection by Resonance-Radiation Pressure. Physical Review Letters 25, 1321. 


\section{References}

(27) Ashkin, A., Dziedzic, J. M., Bjorkholm, J. E., and Chu, S. (1986). Observation of a single-beam gradient force optical trap for dielectric particles. Optics Letters 11, 288-290.

(28) Molloy J. E., and Padgett M. J. (2002). Lights, action: optical tweezers. Contemporary Physics 43, 241-258.

(29) Brau, R. R., Ferrer, J. M., and Lee, H (2007). Passive and active microrheology with optical tweezers. Journal of Optics A: 9, S103.

(30) Crocker, J. C. (1996). Measurement of the hydrodynamic corrections to the brownian motion of two colloidal spheres. The Journal of Chemical Physics 106, 2837-2840.

(31) Gutsche, C, Elmahdy, M., Kegler, K, Semenov, I, Stangner, T, Otto, O, Ueberschär, O, Keyser, U., Krueger, M, Rauscher, M, et al. (2011). Microrheology on (polymer-grafted) colloids using optical tweezers. Journal of Physics: Condensed Matter 23, 184114.

(32) Crocker, J. C., and Grier, D. G. (1994). Microscopic measurement of the pair interaction potential of charge-stabilized colloid. Physical review letters 73, $352-355$.

(33) Tränkle, B., Speidel, M., and Rohrbach, A. (2012). Interaction dynamics of two colloids in a single optical potential. Physical Review E 86, 1-5.

(34) Sainis, S. K., Merrill, J. W., and Dufresne, E. R. (2008). Electrostatic Interactions of Colloidal Particles at Vanishing Ionic Strength. Langmuir 24, 13334-13337.

(35) Gutsche, C, Keyser, U., Kegler, K, Kremer, F, and Linse, P. (2007). Forces between single pairs of charged colloids in aqueous salt solutions. Physical Review E 76, 031403.

(36) Sainis, S. K., Germain, V., and Dufresne, E. R. (2007). Statistics of particle trajectories at short time intervals reveal fN-scale colloidal forces. Physical review letters 99, 018303. 
(37) Sainis, S. K., Germain, V., Mejean, C. O., and Dufresne, E. R. (2008). Electrostatic interactions of colloidal particles in nonpolar solvents: Role of surface chemistry and charge control agents. Langmuir 24, 1160-1164.

(38) Verma, R., Crocker, J., Lubensky, T., and Yodh, A. (1998). Entropic colloidal interactions in concentrated DNA solutions. Physical review letters 81, 4004.

(39) Lin, K.-h., Crocker, J. C., Zeri, A. C., and Yodh, A. (2001). Colloidal interactions in suspensions of rods. Physical review letters 87, 088301.

(40) Mittal, M., Lele, P. P., Kaler, E. W., and Furst, E. M. (2008). Polarization and interactions of colloidal particles in ac electric fields. The Journal of chemical physics 129, 064513.

(41) Lele, P. P., Mittal, M., and Furst, E. M. (2008). Anomalous particle rotation and resulting microstructure of colloids in AC electric fields. Langmuir 24, $12842-12848$.

(42) Park, B. J., and Furst, E. M. (2011). Micromechanics of colloidal aggregates at the oil-water interface. Soft Matter 7, 7683-7688.

(43) Park, B. J., and Furst, E. M. (2011). Attractive interactions between colloids at the oil-water interface. Soft Matter.

(44) Park, B. J., and Furst, E. M. (2008). Optical trapping forces for colloids at the oil-water interface. Langmuir 24, 13383-13392.

(45) Elmahdy, M. M., Gutsche, C., and Kremer, F. (2010). Forces within single pairs of charged colloids in aqueous solutions of ionic liquids as studied by optical tweezers. The Journal of Physical Chemistry C 114, 19452-19458.

(46) Dominguez-Espinosa, G., Synytska, A., Drechsler, A., Gutsche, C., Kegler, K., Uhlmann, P., Stamm, M., and Kremer, F. (2008). Optical tweezers to measure the interaction between poly (acrylic acid) brushes. Polymer 49, 4802-4807.

(47) Rogers, W. B., and Crocker, J. C. (2014). A tunable line optical tweezers instrument with nanometer spatial resolution. Review of Scientific Instruments $85,043704$. 


\section{References}

(48) Koehler, T. P., Brotherton, C. M., and Grillet, A. M. (2011). Comparison of interparticle force measurement techniques using optical trapping. Colloids and Surfaces A: Physicochemical and Engineering Aspects 384, 282-288.

(49) Sugimoto, T., Takahashi, T., Itoh, H., Sato, S.-i., and Muramatsu, A. (1997). Direct Measurement of Interparticle Forces by the Optical Trapping Technique. Langmuir 13, 5528-5530.

(50) Nilsen-Nygaard, J., Sletmoen, M., and Draget, K. I. (2014). Stability and interaction forces of oil-in-water emulsions as observed by optical tweezers a proof-of-concept study. $R S C A d v$. 4, 52220-52229.

(51) Ashkin, A. (1992). Forces of a Single-Beam gradient laser trap on a dielectric sphere in the ray optics regime. Biophysical Journal 61, 569-582.

(52) Neuman, K. C., and Block, S. M. (2004). Optical trapping. Review of Scientific Instruments 75, 2787-2809.

(53) Crocker, J. C., and Grier, D. G. (1998). Interactions and Dynamics in ChargeStabilized Colloids. MRS Bulletin 23, 24-31.

(54) Raudsepp, A., Griffiths, M., Sutherland-Smith, A., and Williams, M. (May 2015). Developing a video tracking method to study interactions between close pairs of optically trapped particles in three-dimensions. Applied Optics Submitted.

(55) Peterman, E. J., Gittes, F., and Schmidt, C. F. (2003). Laser-Induced Heating in Optical Traps. Biophysical Journal 84, 1308 -1316.

(56) Uhrig, K., Kurre, R., Schmitz, C., Curtis, J. E., Haraszti, T., Clemen, A. E.-M., and Spatz, J. P. (2009). Optical force sensor array in a microfluidic device based on holographic optical tweezers. Lab on a chip 9, 661-668.

(57) Bowman, R. W., Gibson, G. M., Linnenberger, A., Phillips, D. B., Grieve, J. A., Carberry, D. M., Serati, S., Miles, M. J., and Padgett, M. J. (2014). Red Tweezers: Fast, customisable hologram generation for optical tweezers. Computer Physics Communications 185, 268-273. 
(58) Gagliardi, L. J. (2006). Electrostatic considerations in nuclear envelope breakdown and reassembly. Journal of electrostatics 64, 843-849.

(59) Behrens, S. H., and Grier, D. G. (2001). Pair interaction of charged colloidal spheres near a charged wall. Physical Review E 64, 050401.

(60) Crank, J., The Mathematics of Diffusion; Oxford science publications; Clarendon Press: 1979.

(61) Dickinson, E., and Miller, Reinhard, Food Colloids Fundmentals of Formulation; The Royal Socity of Chemistry: Cambridge, UK, 2001.

(62) Dalgleish, D. In, 4th; Marcel Dekker: New York, 2004, pp 1-44.

(63) Mason, T. (1999). New fundamental concepts in emulsion rheology. Current Opinion in Colloid 6 Interface Science 4, 231 -238.

(64) Becher, P., Emulsions Theory and Practice, Third; Oxford University Press: New York, 2001.

(65) Dickinson, E., An Introduction to Food Colloids; Oxford Science: Oxford, 1992, p 207.

(66) Taylor, P. (1998). Ostwald ripening in emulsions. Advances in Colloid and Interface Science 75, $107-163$.

(67) Alzagtat, A. A., and Alli, I. (2002). Protein-lipid interactions in food systems: a review. International Journal of Food Sciences and Nutrition 53, 249-260.

(68) Walstra, P, and Pieter, W. (1993). Principles of emulsion formation. Chemical Engineering Science 48, 333-349.

(69) Schultz, S., Wagner, G., Urban, K., and Ulrich, J. (2004). High?Pressure Homogenization as a Process for Emulsion Formation., en Chemical Engineering 83 Technology 27, 361-368.

(70) Floury, J., Desrumaux, A., and Lardières, J. (2000). Effect of high-pressure homogenization on droplet size distributions and rheological properties of model oil-in-water emulsions. Innovative Food Science 6 Emerging Technologies 1, $127-134$. 


\section{References}

(71) Guzey, D., and McClements, D. J. (2006). Formation, stability and properties of multilayer emulsions for application in the food industry. Advances in Colloid and Interface Science 128-130, 227-248.

(72) Ogawa, S., Decker, E. A., and McClements, D. J. (2003). Production and Characterization of $\mathrm{O} / \mathrm{W}$ Emulsions Containing Cationic Droplets Stabilized by LecithinChitosan Membranes. Journal of Agricultural and Food Chemistry 51, 2806-2812.

(73) Surh, J., Gu, Y. S., Decker, E. A., and McClements, D. J. (2005). Influence of Environmental Stresses on Stability of O/W Emulsions Containing Cationic Droplets Stabilized by SDSFish Gelatin Membranes. J. Agric. Food Chem. 53, 4236-4244.

(74) Dickinson, E. (1997). Properties of Emulsions Stabilized with Milk Proteins: Overview of Some Recent Developments. Journal of Dairy Science 80, 26072619.

(75) Thompson, A., Boland, M., and Singh, H., Milk Proteins: From Expression to Food, en; Academic Press: 2008, p 562.

(76) de Feijter, J., and Benjamins, J (1982). Soft-particle model of compact macromolecules at interfaces. Journal of Colloid and Interface Science 90, 289-292.

(77) Kim, H.-J., Decker, E. A., and McClements, D. J. (2004). Influence of Free Protein on Flocculation Stability of $\beta$-Lactoglobulin Stabilized Oil-in-Water Emulsions at Neutral pH and Ambient Temperature. Langmuir 20, 1039410398.

(78) Zhai, J., Wooster, T. J., Hoffmann, S. V., Lee, T.-H., Augustin, M. A., and Aguilar, M.-I. (2011). Structural rearrangement of $\beta$-lactoglobulin at different oil-water interfaces and its effect on emulsion stability. Langmuir 27, 92279236.

(79) Singh, H. (2011). Aspects of milk-protein-stabilised emulsions. Food Hydrocolloids 25, 1938-1944. 
(80) Zhai, J., Miles, A. J., Pattenden, L. K., Lee, T.-H., Augustin, M. A., Wallace, B. A., Aguilar, M.-I., and Wooster, T. J. (2010). Changes in $\beta$-lactoglobulin conformation at the oil/water interface of emulsions studied by synchrotron radiation circular dichroism spectroscopy. Biomacromolecules 11, 2136-2142.

(81) Euston, S. R., Hirst, R. L., and Hill, J. P. (1999). The emulsifying properties of $\beta$-lactoglobulin genetic variants A, B and C. Colloids and Surfaces B: Biointerfaces 12, $193-202$.

(82) Elofsson, U. M., Paulsson, M. A., and Arnebrant, T. (1997). Adsorption of $\beta$ Lactoglobulin A and B in Relation to Self-Association: Effect of Concentration and pH. Langmuir 13, 1695-1700.

(83) Elofsson, U. M., Paulsson, M. A., and Arnebrant, T. (1997). Adsorption of $\beta$-lactoglobulin A and B: Effects of ionic strength and phosphate ions. Colloids and Surfaces B: Biointerfaces 8, 163-169.

(84) Guzey, D., and McClements, D. J. (2006). Influence of Environmental Stresses on $\mathrm{O} / \mathrm{W}$ Emulsions Stabilized by $\beta$-Lactoglobulin-Pectin and $\beta$ Lactoglobulin-Pectin-Chitosan Membranes Produced by the Electrostatic Layer-by-Layer Deposition Technique. Food Biophysics 1, 30-40.

(85) Dickinson, E. (1989). Surface and emulsifying properties of caseins. J. Dairy Res 56, 471-477.

(86) Singh, H., and Sarkar, A. (2011). Behaviour of protein-stabilised emulsions under various physiological conditions. Advances in Colloid and Interface Science 165, 47-57.

(87) McClements, D. J. (2007). Critical review of techniques and methodologies for characterization of emulsion stability. Critical reviews in food science and nutrition 4\%, 611-49.

(88) Alexey, K. (1998). Thermodynamic and theoretical aspects of emulsions and their stability. Current Opinion in Colloid 8 Interface Science 3, 270-275. 


\section{References}

(89) Lee, M. H., Reich, D. H., Stebe, K. J., and Leheny, R. L. (2009). Combined passive and active microrheology study of protein-layer formation at an air-water interface. Langmuir 26, 2650-2658.

(90) Desrumaux, A., and Marcand, J. (2002). Formation of sunflower oil emulsions stabilized by whey proteins with high-pressure homogenization (up to 350 $\mathrm{MPa}$ ): effect of pressure on emulsion characteristics. International journal of food science \& technology 37, 263-269.

(91) Robin, O, Blanchot, V, Vuillemard, J., and Paquin, P (1992). Microfluidization of dairy model emulsions. I. Preparation of emulsions and influence of processing and formulation on the size distribution of milk fat globules. Le Lait 72, 511-531.

(92) Tornberg, E (1980). Functional characteristics of protein stabilized emulsions: emulsifying behavior of proteins in a sonifier. Journal of Food Science 45, 1662-1668.

(93) Messens, W., Van Camp, J., and Huyghebaert, A. (1997). The use of high pressure to modify the functionality of food proteins. Trends in Food Science \& Technology 8, 107-112.

(94) Galazka, V. B., Dickinson, E., and Ledward, D. A. (2000). Influence of high pressure processing on protein solutions and emulsions. Current opinion in colloid E interface science 5, 182-187.

(95) Qi, P. X., Wickham, E. D., and Garcia, R. A. (2014). Structural and Thermal Stability of $\beta$-Lactoglobulin as a Result of Interacting with Sugar Beet Pectin. Journal of agricultural and food chemistry 62, 7567-7576.

(96) Ward, A. D., Berry, M. G., Mellor, C. D., and Bain, C. D. (2006). Optical sculpture: controlled deformation of emulsion droplets with ultralow interfacial tensions using optical tweezers. Chemical Communications, 4515-4517.

(97) Bauer, W.-A. C., Kotar, J., Cicuta, P., Woodward, R. T., Weaver, J. V. M., and Huck, W. T. S. (2011). Microfluidic production of monodisperse functional $\mathrm{o} / \mathrm{w}$ droplets and study of their reversible $\mathrm{pH}$ dependent aggregation behavior. Soft Matter 7, 4214-4220. 


\section{References}

(98) Marichal, M. J. A. (2014). Manipulating Sodium Caseinate Behaviour at the Interface: Applications for Concentrated Emulsion Formulation.

(99) Dagastine, R. R., Manica, R. R. R. R., Carnie, S. L., Chan, D. Y. C., Stevens, G. W., and Grieser, F. (2006). Dynamic Forces Between Two Deformable Oil Droplets in Water. Science 313, 210-213.

(100) Gunning, A. P., Kirby, A. R., Wilde, P. J., Penfold, R., Woodward, N. C., and Morris, V. J. (2013). Probing the role of interfacial rheology in the relaxation behaviour between deformable oil droplets using force spectroscopy. Soft Matter 9, 11473-11479. 